\title{
Reducing interferences in glow discharge spectroscopies using transient operation and collision -induced dissociation
}

Glen Paul Jackson

West Virginia University

Follow this and additional works at: https://researchrepository.wvu.edu/etd

\section{Recommended Citation}

Jackson, Glen Paul, "Reducing interferences in glow discharge spectroscopies using transient operation and collision -induced dissociation" (2002). Graduate Theses, Dissertations, and Problem Reports. 1628. https://researchrepository.wvu.edu/etd/1628

This Dissertation is protected by copyright and/or related rights. It has been brought to you by the The Research Repository @ WVU with permission from the rights-holder(s). You are free to use this Dissertation in any way that is permitted by the copyright and related rights legislation that applies to your use. For other uses you must obtain permission from the rights-holder(s) directly, unless additional rights are indicated by a Creative Commons license in the record and/ or on the work itself. This Dissertation has been accepted for inclusion in WVU Graduate Theses, Dissertations, and Problem Reports collection by an authorized administrator of The Research Repository @ WVU.

For more information, please contact researchrepository@mail.wvu.edu. 


\title{
Reducing Interferences in Glow Discharge Spectroscopies Using Transient Operation and Collision-Induced Dissociation
}

\author{
Glen P. Jackson \\ Dissertation submitted to the \\ Eberly College of Arts and Sciences \\ at West Virginia University \\ in partial fulfillment of the requirements for the degree of \\ Doctor of Philosophy \\ in \\ Chemistry \\ Fred L. King, Ph.D., Chair \\ Ronald B. Smart, Ph.D. \\ Aaron Timperman, Ph.D. \\ Bjorn Soderberg, Ph.D. \\ Earl Scime, Ph.D. \\ Douglas C. Duckworth, Ph.D. \\ Morgantown, West Virginia \\ 2002
}

Keywords: Pulsed glow discharge, Mass Spectrometry, Ionizing plasma, Recombining plasma, Collision-induced dissociation, Quadrupole ion trap

Copyright 2002 Glen P. Jackson 


\section{Abstract \\ Reducing Interferences in Glow Discharge Spectroscopies Using Transient Operation and Collision-Induced Dissociation}

\section{Glen P. Jackson}

Plasma sources for optical spectroscopy and mass spectrometry have provided scientists unprecedented knowledge about the fundamental properties and behavior of matter. Analytical chemists are finding increasingly numerous and diverse applications for plasma sources coupled with optical or mass spectrometric detection methods, such that there is hardly a substance on earth, or collected from other planets, that has not at one time been characterized by these methods. As informative and useful as these methods are; however, there is still considerable room for improvement. The areas in need of improvement include detection limits, analysis times, number of required standards, and quantitative accuracy and precision. The accuracy and precision of quantitative analyses by mass spectrometry and optical emission spectroscopy are often dependent on the presence of interferences. Interferences are defined as a measured signal response to a species at the spectral position of interest for the analyte that is not caused by the analyte. In optical emission experiments, interference can occur $\mathrm{f}$ two elements emit light at the same nominal wavelength, and in mass spectrometry, interferences can occur wherever two different species (molecular or atomic) exhibit the same nominal $\mathrm{m} / \mathrm{z}$ ratio.

Because the presence of spectral interferences can deleteriously affect the integrity of reported results, methods are required to reduce, eliminate, or otherwise compensate for the interfering signals. This study focuses on two such methods for reducing spectral interferences. The first method is to operate the glow discharge source in a transient mode in order to spatially and temporally resolve analyte and interfering signals. The second method is to employ collision-induced dissociation in a quadrupole ion trap in order to reduce molecular interferences in atomic mass spectrometry. 
Optical and mass spectrometric studies are described in which the spatio-temporal evolution of the pulsed glow discharge is elucidated. The sequences of events following voltage termination of a 5-millisecond pulse are studied in detail, especially concerning reactions leading to the generation of analyte ions and excited states. The addition of nitrogen as a diagnostic tool is serendipitously found to enhance the temporal characteristics that separate the plasma gas emissions and ions from the sputtered analyte emissions and ions. This could lead to future applications for time-gated detection methods in optical and mass spectrometric analyses that minimize interferences due to plasma species.

Collision-induced dissociation of strongly bound molecular interferences in a quadrupole ion trap is examined as a potential means for the removal of these interferences. Methods for determining the dissociation rates and factors affecting the dissociation rates and efficiencies are also investigated, along with a method for determining the bond dissociation energy of diatomic ions. Mathematical simulations and fundamental considerations show that reaction rates and thermodynamic data can be determined using neon as the bath gas instead of the more-commonly-used helium. The larger mass of neon is necessary for promoting the dissociation of strongly bound diatomic ions such as lanthanum oxide at $\sim 860 \mathrm{~kJ} \mathrm{~mol}^{-1}$. Such collision-induced dissociation in the quadrupole ion trap is also shown to be possible while simultaneously trapping bare metal ions at the same nominal mass. Various methods are investigated for applying the excitation amplitudes that cause the dissociation of these diatomic ions, and a method is proposed for promoting dissociation during the axial modulation event that 
generates the mass spectra. These latter techniques could eventually lead to the ability to acquire purely elemental mass spectra with minimal operator intervention. 
In loving memory of my grandfather

Frederick John Hickman 


\section{Acknowledgements}

I am very grateful to my advisor, Dr. Fred L. King, for the numerous ways in which he has enriched my graduate learning experience. As well as being an excellent teacher in the classroom and in the lab, he also believed in a broader graduate learning experience. His collaborative links with Oak Ridge National Laboratory (ORNL) in conjunction with his encouragement, allowed me to spend the latter half of my studies in Oak Ridge where I enjoyed a rewarding internship. Similarly, I am extremely appreciative of my mentor at ORNL, Dr. Douglas C. Duckworth, who took me under his wing and taught me a great deal about conducting research at a government laboratory. Together, Dr. King and Dr. Duckworth have spent untold hours editing manuscripts, checking presentations, and offering advice on experiments. I will always be thankful for their guidance and support.

I wish to express my gratitude to the other members of the King group at WVU for their assistance, and to my other peers in Morgantown for all the fun times. Much of the data for the Chapters 2 and 3 was collected with Dr. Cris Lewis, and I learned a lot about commitment and dedication during that time. Thanks Cris. I'm also grateful to my committee members: Dr. Earle E. Scime, Dr. Ronald B. Smart, Dr. Bjorn Soderberg and Dr. Aaron Timperman. I appreciate the time they took to read my proposal and dissertation and to sit through the presentations of each. I am also thankful to Dr. Steve Doorn and Dr. Vahid Majidi at Los Alamos National Laboratory for a collaborative project conducted during my time at WVU. I also appreciate Dr. H. O. Finklea, Dr.

Demitri Brevnov and Maria-Rosa Valero-Aracama for their assistance with the deconvolution process used in Chapters 2 and 4. 
At ORNL I was made to feel very welcome by all the members of the Isotope and Chemical Mass Spectrometry group, and I am indebted to Eddie McBay and Group Leader Dr. Lee Riciputi for all their help. Thanks also to all the other staff members in building 5505, especially for the fine lunchtime cuisine on Wednesdays. Special thanks to Dr. John Gibson and Dr. Doug Goeringer for their collaborative support in several projects during my time at ORNL. They were both particularly patient and understanding in answering all my questions. I also wish to acknowledge funding support from the Division of Chemical Sciences, Geosciences, and Biosciences, Office of Basic Energy Sciences, U.S. Department of Energy, under contract DE-AC05-00OR22725 with Oak Ridge National Laboratory, managed and operated by UT-Battelle, LLC. The Eberly College of Arts and Sciences at WVU and the Chemistry Department at WVU are also greatly appreciated for their financial support.

On a more personal note, I wish to express my deepest gratitude to my mother Sue Jackson, my father Roy Jackson, and my brother Craig Jackson for their love and support over the years. My mother has been a particularly strong influence in my life, and I am still discovering ways in which I benefit from her love and selflessness. They, and the rest of my very loving family, have been very understanding of my decision to live and work abroad. A very special thank you to my in-laws, Karl and Mary, and the entire Gdovka family, who have made me feel so at home and welcome in America. They have been incredibly supportive, and I appreciate them welcoming me into their family so whole-heartedly.

Finally, I would like to express my appreciation for my wife Linda. Before undertaking this dissertation I had little idea just how much I would depend on her to help 
me through. Now I know, and I wish I could express just how much she means to me. She was more than understanding about all the hours I spent cocooned in our office and even had the patience to help edit significant portions of the work. She gives me a perspective and meaning to my life in a way that brings a healthy balance to my existence. Our love is the greatest motivation of all. 


\section{Table of Contents}

Page

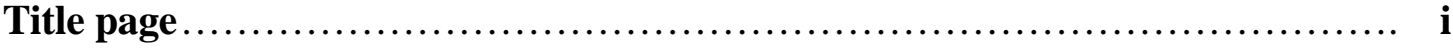

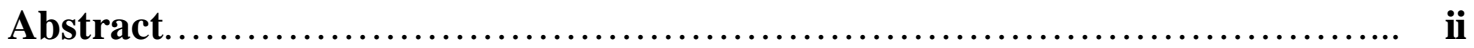

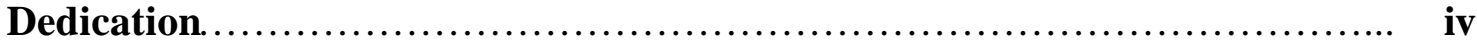

Acknowledgements .................................................. vi

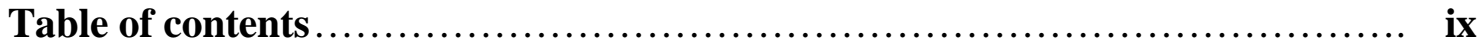

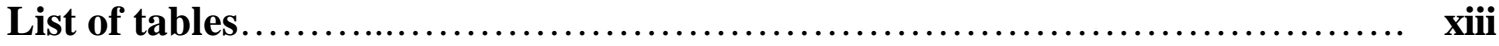

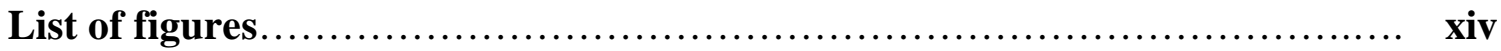

1. Introduction. ...................................................... 1

1.1. Pulsed glow discharges: a historical perspective .................. 7

1.2. Analytical pulsed glow discharges for optical spectroscopy ........... 14

1.3. Analytical pulsed glow discharges for mass spectrometry ............. 16

1.4. References........................................................ 20

2. Metastable argon atoms in the pulsed glow discharge $\ldots \ldots \ldots \ldots \ldots \ldots \ldots \ldots$

2.1. Introduction. ..................................................... 28

2.2. Experimental............................................. 31

2.2.1. Emission experiments.................................. 33

2.2.2. Absorbance measurements................................. 33

2.2.3. Laser-induced atomic fluorescence........................... 34

2.3. Results and discussion $\ldots \ldots \ldots \ldots \ldots \ldots \ldots \ldots \ldots \ldots \ldots \ldots \ldots \ldots \ldots \ldots \ldots \ldots \ldots \ldots, \quad 36$

2.3.1. ${ }^{3} \mathrm{P}_{2}$ Argon atom metastables: Population via radiative decay........ 40

2.3.2. ${ }^{3} \mathrm{P}_{2}$ Argon atom metastables: Absorption...................... 43

2.3.3. ${ }^{3} \mathrm{P}_{0}$ Argon atom metastables: Population via radiative decay........ 46

2.3.4. ${ }^{3} \mathrm{P}_{0}$ Argon atom metastables: Absorption and laser-induced fluorescence measurements....................................... 48

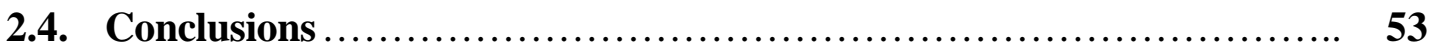




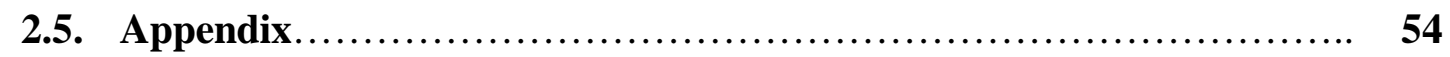

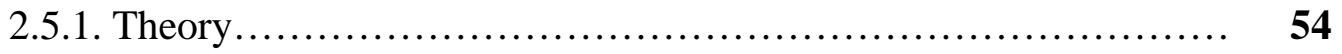

2.5.2. Application of theory .................................... 54

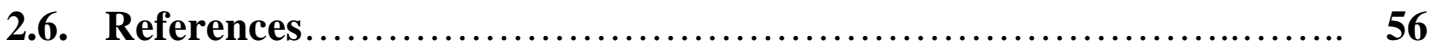

3. Bulk plasma properties of the pulsed glow discharge ........................ 59

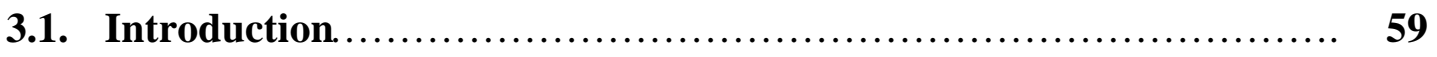

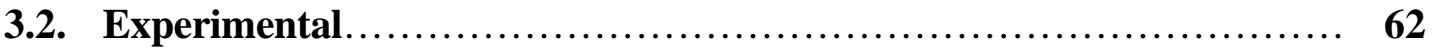

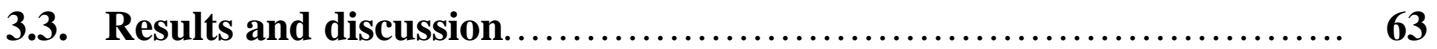

3.3.1. Argon atom emissions.......................................... 63

3.3.1.1. $3 p^{5} 5 \mathrm{p}-3 \mathrm{p}^{5} 4 \mathrm{~s}$ transitions................................ 67

3.3.1.2. $3 p^{5} 7 s-3 p^{5} 4 p$ transitions............................... 73

3.3.1.3. $3 p^{5} 4-7 \mathrm{~d}-3 \mathrm{p}^{5} 4 \mathrm{p}$ transitions.............................. 74

3.3.1.4. $3 p^{5} 4 p-3 p^{5} 4$ s transitions.................................... 75

3.3.2. Deviations from thermodynamic equilibrium..................... 76

3.3.2.1. Departure from the Maxwell balance....................... $\quad \mathbf{7 6}$

3.3.2.2. Departure from the Saha-Boltzmann balance................ 79

3.3.2.3. Departure from the Planck balance......................... 87

3.3.2.4. Additional factors contributing to TE departure............... 87

3.3.3. Transition from an ionizing- to a recombining plasma.............. $\quad \mathbf{8 8}$

3.3.4. Phase transitions at the pulse-onset............................ 90

3.3.5. Argon ion emissions ......................................... 95

3.3.6. The mechanism of electron-ion recombination................... 100

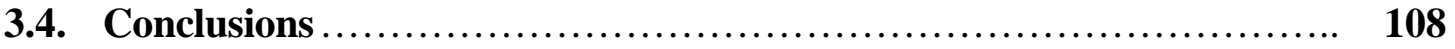

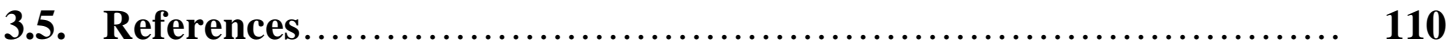

4. Effect of nitrogen on an argon pulsed glow discharge ................... 116

4.1. Introduction ................................................... 116

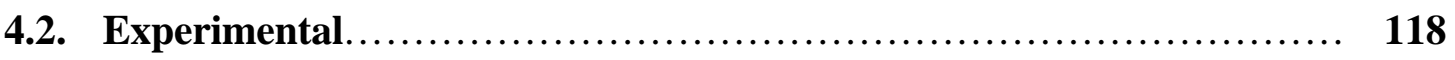

4.2.1. Optical experiments..................................... 119

4.2.2. Time-of-Flight mass spectrometry (ToF-MS) experiments........... 120 
4.3. Results and discussion ......................................... 123

4.3.1. Bath gas temperature measurements.......................... 123

4.3.2. $\mathrm{N}_{2}$ Quenching of metastable argon atoms....................... 129

4.3.3. $\mathrm{N}_{2}$ effects on post-pulse recombination........................ $\quad \mathbf{1 3 8}$

4.3.3.1. Effect of $\mathrm{N}_{2}$ on argon ions............................. 141

4.3.3.2. Effect of $\mathrm{N}_{2}$ on electrons.............................. 142

4.3.4. Effect of $\mathrm{N}_{2}$ on analyte signals.............................. 145

4.3.4.1. Fe atom emissions..................................... 147

4.3.4.2. Fe ion signals...................................... 152

4.4. Conclusions ........................................................ 166

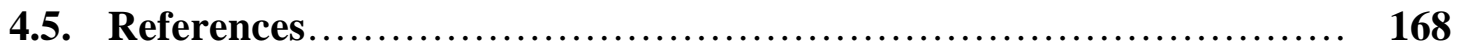

5. Gas Phase Reactions of $\mathrm{U}^{+}$and $\mathrm{U}^{2+}$ with $\mathrm{O}_{2}$ and $\mathrm{H}_{2} \mathrm{O}$ in a Quadrupole Ion Trap..................................................................... 175

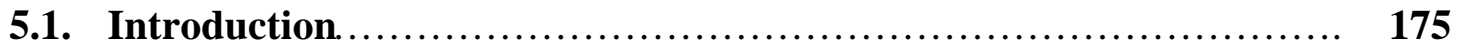

5.2. Experimental apparatus and method of measurement ................. 177

5.3. Results and discussion............................................. 179

5.3.1. Kinetic energy considerations.............................. 179

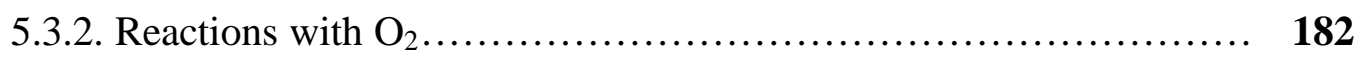

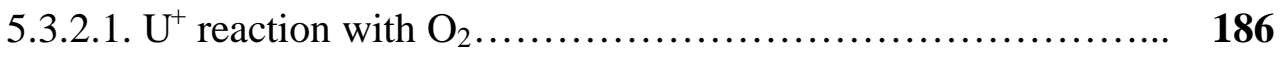

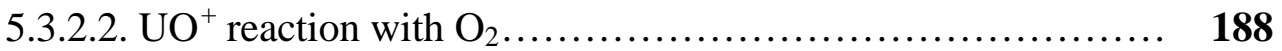

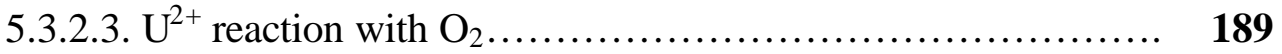

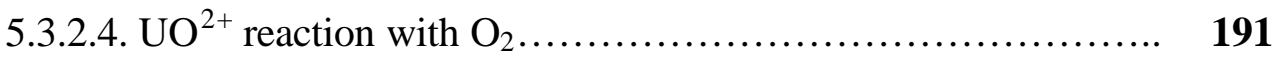

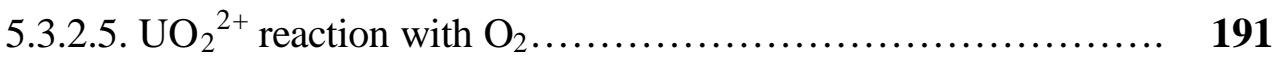

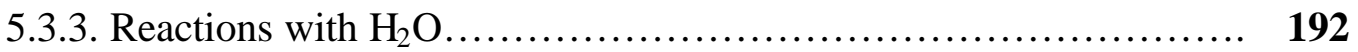

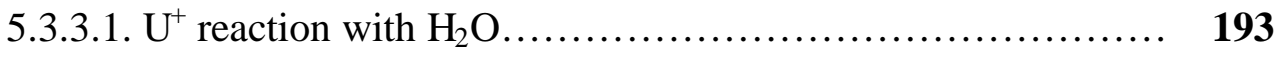

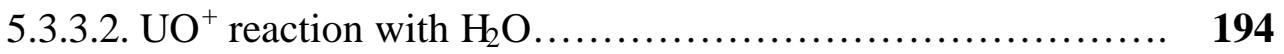

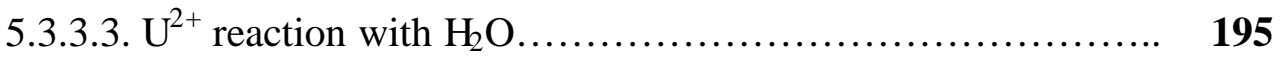

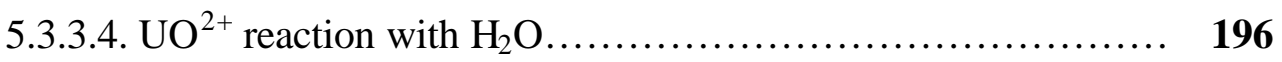

5.4. Conclusions .......................................................... 197

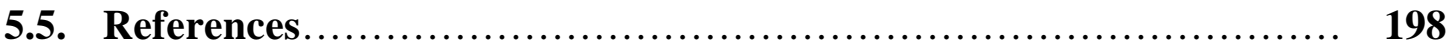


6. Collision-Induced Dissociation of Lanthanide Oxide Ions in Quadrupole Ion Traps: Effects of Bond Strength and Mass.................... 201

6.1. Introduction. ............................................... 201

6.2. Experimental............................................... 204

6.2.1. Method development...................................... 206

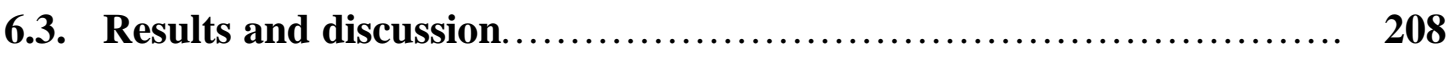

6.3.1. Bond strength effects on dissociation rate..................... 210

6.3.2. Mass effects on dissociation rate............................ 210

6.3.3. Determining dissociation energy of 'unknowns' ................. 213

6.3.4. Bond strength and mass effects............................ 214

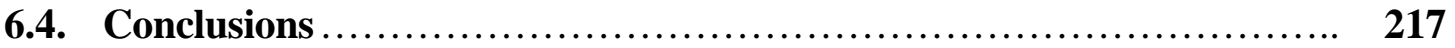

6.5. References................................................ 219

7. Removal of Molecular Interferences in Elemental Quadrupole Ion Trap Mass Spectrometry Using Collision Induced Dissociation.......... 221

7.1. Introduction................................................. 221

7.2. Experimental................................................ 224

7.3. Results and discussion...................................... 226

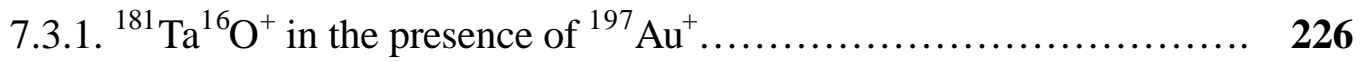

7.3.2. ${ }^{154} \mathrm{Gd}^{16} \mathrm{O}^{+}$and ${ }^{155} \mathrm{Gd}^{16} \mathrm{O}^{+}$in the presence of ${ }^{170} \mathrm{Yb}^{+}$and ${ }^{171} \mathrm{Yb}^{+} \ldots \ldots . .232$

7.3.3. ${ }^{63,63} \mathrm{Cu}_{2}{ }^{+}{ }^{63,65} \mathrm{Cu}_{2}{ }^{+}$, and ${ }^{65,65} \mathrm{Cu}_{2}{ }^{+}$in the presence of ${ }^{126} \mathrm{Te}^{+},{ }^{128} \mathrm{Te}^{+}$,

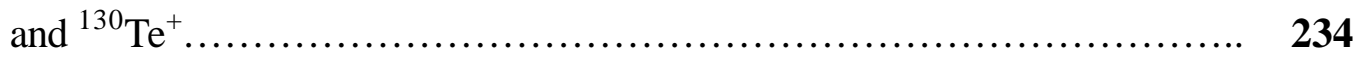

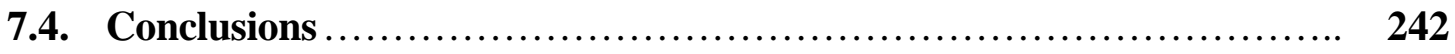

7.5. References............................................... 243

Curriculum vitae.................................................. 247 


\section{List of Tables}

Page

3.1 Spectroscopic data for argon atom lines examined in this study........... 65

3.2 Spectroscopic data for argon ion lines examined in this study............ 66

4.1 Settings used for the PGD-ToF-MS experiments.................... 122

5.1 Thermochemical data of species of interest......................... 183

5.2 Reactions of singly charged uranium species with molecular oxygen and water........................................................ 184

5.3 Reactions of doubly charged uranium species with molecular oxygen and

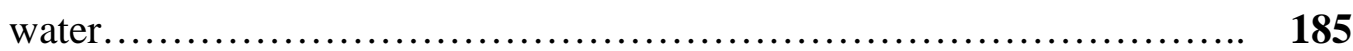

6.1 Dissociation energy and masses of the oxide ions used................ 211

6.2 Dissociation energy determinations of 'unknowns' using the empirical and theoretical correction terms for mass effects...................... 212

7.1 Measured isotopic abundances for some Te ions before and after the application of broadband excitation to remove interfering $\mathrm{Cu}_{2}{ }^{+}$ions......... 


\section{List of Figures}

Page

2.1 Schematic diagram of apparatus used for the optical experiments......... 32

2.2 Energy level scheme and transitions for the argon atom metastables....... 37

2.3 Comparison of various optical responses for the transition at $811.5 \mathrm{~nm} \ldots . .39$

2.4 Two-dimensional images of argon atom emission at $811.5 \mathrm{~nm} \ldots \ldots \ldots \ldots . \quad 41$

2.5 Two-dimensional images of argon atom absorption at $811.5 \mathrm{~nm} \ldots \ldots \ldots \ldots . \mathbf{4 5}$

2.6 Two-dimensional images of argon atom emission at $794.8 \mathrm{~nm} \ldots \ldots \ldots \ldots \ldots \quad 47$

2.7 Two-dimensional images of the ${ }^{3} \mathrm{P}_{0}$ metastable state at $4.0 \mathrm{~ms}$ by absorption at $794.8 \mathrm{~nm}$ and laser-induced fluorescence...................

2.8 Ratio of afterpeak/plateau values for the ${ }^{3} \mathrm{P}_{0}$ metastable state by absorption at $794.8 \mathrm{~nm}$ and laser-induced fluorescence................ 51

3.1 Energy level diagrams of the argon atom and argon ion................ 64

3.2 Temporal traces for selected argon atom transitions at different distances above the cathode...................................................

3.3 Optical emission signals for argon atom lines in the steady-state and

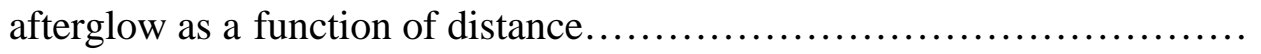

3.4 Plots to determine the electron temperature from the Saha response and Boltzmann response of argon atom lines to voltage termination.............

3.5 Plots to determine the electron temperature from the Saha response and Boltzmann response of copper atom lines to voltage termination...........

3.6 Examples of electron excitation mechanisms at different electron number densities in an ionizing plasma...................................

3.7 Examples of electron de-excitation mechanisms at different electron number densities in a recombining plasma............................

3.8 Plots of the afterpeak/steady-state and afterpeak/prepeak emission ratios as a function of the energy level of the emitting level at different sampling distances above the cathode..........................................

3.9 Plot of the emission intensity of the argon ion line at $476.4 \mathrm{~nm}$ as a function of time. 
3.10 Plot of argon ion emissions for several lines as a function of distance.......

3.11 Two-dimensional maps of the normalized argon ion emission at $476.4 \mathrm{~nm}$.

3.12 Argon atom decay rates as a function of the upper energy level of the emitting species.

4.1 Schematic of the Pulsed Glow Discharge Time-of-Flight Mass Spectrometer (PGD-ToF-MS) apparatus

4.2 Time-averaged emission spectrum of the first negative column of $\mathrm{N}_{2}{ }^{+}$in a PGD and Boltzmann plot for the determination of $\mathrm{T}_{\text {rot }}$ from the emission spectrum.

4.3 Time-averaged rotational temperatures in the PGD determined from the emission spectra of $\mathrm{N}_{2}{ }^{+}$....

4.4 Schematic to show the energy transfer reactions between argon metastables and molecular nitrogen, and the resulting emissions (nm) used to characterize the quenching process.................................

4.5 Time-averaged rotational spectrum of the $(0,0)$ band of the second positive emission of nitrogen, with the band head at $337.1 \mathrm{~nm}$

4.6 Comparison of different nitrogen molecular emissions

4.7 Temporal emission profiles at $337.1 \mathrm{~nm}$ for the $(0,0)$ band of the second positive column.

4.8 Effect of nitrogen on the post-pulse population of ${ }^{3} \mathrm{P}_{2}$ metastables

4.9 Plot of the afterpeak/steady-state absorption ratios for the ${ }^{3} \mathrm{P}_{2}$ metastable levels of argon before and after the addition of $1 \% \mathrm{~N}_{2}$

4.10 Emission intensity at $811.5 \mathrm{~nm}$ versus time to show the effect of nitrogen on the afterpeak emissions in an argon PGD.

4.11 Argon ion signal at $\mathrm{m} / \mathrm{z}=40$ versus time to show the effect of nitrogen on the steady-state and post-pulse number densities. ...

4.12 Emission from the $811.5 \mathrm{~nm}$ line as a function of time to show the effect of decreasing nitrogen in the discharge.

4.13 Effect of $1 \%$ nitrogen on sputtered atom emissions.

4.14 Emission intensity at $4.9 \mathrm{~ms}$ at $371.99 \mathrm{~nm}$ as a function of distance above the cathode to show the uniform reduction in signal with $1 \% \mathrm{~N}_{2}$ added..... 
4.15 Emission intensity at $4.9 \mathrm{~ms}$ at $811.5 \mathrm{~nm}$ as a function of distance above the cathode to show the (lack of) effect of $1 \% \mathrm{~N}_{2}$ addition.....

4.16 ${ }^{56} \mathrm{Fe}^{+}$ion signals as a function of sampling height above the cathode to show the effect of $1 \%$ nitrogen on the ionization of sputtered metal atoms.

4.17 Attenuation factors for the steady-state signals of different ions in response to the addition of $1 \%$ nitrogen.................................

4.18 Attenuation factors for the steady-state signals of $\mathrm{Ar}^{2+}$ and $\mathrm{ArH}^{+}$in response to the addition of $1 \%$ nitrogen...............................

4.19 Selected ion signals as a function of time to show the effect of added nitrogen.

4.20 ${ }^{56} \mathrm{Fe}^{+}$signal, determined by ToF-MS, as a function of distance to show the effect of $1 \%$ nitrogen on ionization in the afterglow

4.21 Emission intensity at $371.99 \mathrm{~nm}$ for the Fe I line as a function of distance to show the effect of $1 \%$ nitrogen on the afterglow

4.22 Iron ion absorbance at $259.93 \mathrm{~nm}$ as a function of distance above the cathode to show the effect of $1 \%$ nitrogen addition on the afterglow.

4.23 Comparison of the afterpeak emissions for Ar I and Fe I with no nitrogen added.

4.24 Comparison of the afterpeak emissions for $\mathrm{Ar} \mathrm{I}$ and $\mathrm{Cu} \mathrm{I}$ with no nitrogen added.

5.1 Simulated root mean square (RMS) ion kinetic energy of uranium ions as a function of Mathieu parameter $\mathrm{q}_{\mathrm{z}}(1 \mathrm{mT}$ Torr $\mathrm{Ne})$

5.2 Measured reaction rates and pathway for the reactions of $\mathrm{U}^{2+}$ and $\mathrm{U}^{+}$with $\mathrm{O}_{2}$

5.3 Ion signal intensities versus time for the reaction of $\mathrm{U}^{+}$with $\mathrm{O}_{2}$

5.4 Measured reaction rates of $\mathrm{U}^{+}$and $\mathrm{UO}^{+}$with $\mathrm{O}_{2}$ versus relative number density of added $\mathrm{O}_{2}$

5.5 Measured reaction rates versus relative number density of added $\mathrm{O}_{2}$ to determine the rate constants for the reactions of $\mathrm{U}^{2+}$ and $\mathrm{UO}^{2+}$ with $\mathrm{O}_{2} \ldots$.

5.6 Reaction pathways for the reactions of $\mathrm{U}^{2+}$ and $\mathrm{U}^{+}$with gaseous $\mathrm{H}_{2} \mathrm{O} \ldots \ldots$. 
6.1 Comparison of dissociation rates as a function of resonance excitation voltage for $\mathrm{LaO}^{+}$and $\mathrm{TaO}^{+}$

6.2 Plot of the dissociation rates as a function of bond dissociation energy for oxide ions having the same nominal mass.

6.3 Plot of the dissociation rate versus oxide ion mass for compounds having the same nominal dissociation energy.

6.4 Plot of dissociation rates as a function of bond dissociation a) before and b) after correction for mass effects

7.1 Ion signals obtained for the collision-Induced dissociation of $\mathrm{TaO}^{+} \ldots$

7.2 Plot of ion signal versus excitation time to show the effect of excitation amplitude on the steady state signals of ${ }^{181} \mathrm{Ta}^{+}$at $\mathrm{m} / \mathrm{z} 181$ and

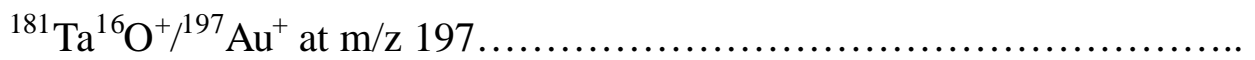

7.3 Plot of the ion signal versus time to show the attenuation rate at $\mathrm{m} / \mathrm{z} 197$

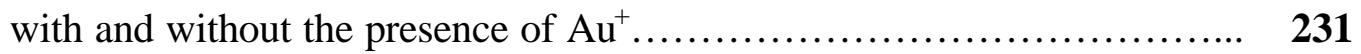

7.4 Isotopes of $\mathrm{Yb}^{+}$with $\mathrm{GdO}^{+}$interfering at $\mathrm{m} / \mathrm{z} 170$ and $171 \ldots \ldots \ldots \ldots \ldots \ldots .233$

7.5 Broadband dissociation of $\mathrm{Cu}_{2}{ }^{+}$isotopologues in the presence of $\mathrm{Te}^{+}$ isotopes.........................................................

7.6 Schematic to represent the CID of a diatomic ion using a supplementary excitation frequency with axial modulation...........................

7.7 Mass spectrum obtained when $\mathrm{Cu}_{2}{ }^{+}$is subjected to resonance excitation at $110 \mathrm{kHz}$ during axial modulation. 


\section{Chapter 1}

\section{$\underline{\text { Introduction }}$}

Mass spectrometry is probably the most versatile, informative, ensitive, and ubiquitous of all instrumental methods of analysis. Almost any substance known to man can and has been analyzed by mass spectrometry, and novel applications are still being found to this day. Mass spectrometry provides information about the quantity of molecules or atoms in a given substance, the isotopic ratios of the constituent atoms, the energy required to withdraw or attach an electron from each of the constituents in the sample, the structure of molecules, the energy required to fragment or dissociate molecules, the size of molecules, and the chemical reactivity of ions with other ions or neutrals. ${ }^{1}$ This information can also be acquired with samples as little as a few nanograms in mass. Such are far-reaching possibilities of mass spectrometry that Joseph J. Thompson—one of the pioneers of mass spectrometry-once noted: ${ }^{2}$

I feel sure that there are many problems in chemistry which could be solved with far greater ease by this than any other method. The method is surprisingly sensitive-

more so even than that of spectrum analysis, requires an infinitesimal amount of material, and does not require this to be specially purified; the technique is not difficult if appliances for producing high vacua are available. 
Many analytical che mists still feel this way almost a hundred years later, but the determination of the masses of the elements and of molecular compounds has not always found such wide support. Almost a hundred years before Thompson's famous book, Andrew Ure had declared that "the body and soul of chemistry did not reside in the knowledge of the combining weights of substances. The characteristics of chemical genius were to discover new elementary bodies, new qualities, and to arrange under general laws the phenomena of corpuscular action. Qualitative research," he quipped, "should be favored over quantitative; atomic weight determinations are a banuasic activity." Little could Ure have known that atoms had the propensity for isotopic differentiation, and that the quantitative analysis, by mass spectrometry, of each of the composite isotopes with respect to one another could find such invaluable applications as in medicine, geology, geochronology, forensic science, and atomic-energy-related fields. Other notable applications can be found in biology, petrology, the semiconductor industry, cosmetics, pharmaceuticals, and other health-related fields.

Like any other analytical technique, mass spectrometry is not without its shortfalls. Although mass spectra are considerably less complicated than optical spectra, there are often situations in which two very different substances in a sample have very similar masses (or, more accurately, mass-to-charge ratios). With powerful resolving power, one can distinguish between the very small differences in mass between two species, but without this capability, one is left with what is known as spectral interference - the overlapping of two different peaks at the same nominal mass.

In quantitative determinations, such isobaric interferences can be severely detrimental to the accuracy of the reported results. It is therefore desirable that isobaric 
interferences be reduced, or corrected for, as much as possible. There are numerous methods currently available with which to elucidate spectral interferences and can be divided into two arbitrary categories. The first is to reduce the interferences before the mass-spectrometric system (i.e. in the handling, preparation, separation, and ionization of the sample). The second ploy is to resolve or remove the interferences within the mass spectrometric system. The chosen method(s) for reducing interferences depends on the origin of the interfering species. For inorganic determinations using plasma sources (the object of this work), interferences are most often caused by molecular impurities. Such impurities may be present in the support gas, or may enter the apparatus via leaks in the vacuum system, desorption from the chamber walls, or from the sample itself. Interferences can arise from molecular contaminants themselves (e.g. $\mathrm{CO}^{+}, \mathrm{N}_{2}^{+}, \mathrm{N}_{3}^{+}$), or from polyatomic species formed between the contaminants and the discharge (e.g. $\mathrm{ArC}^{+}$, $\mathrm{ArN}^{+}, \mathrm{ArO}^{+}$). Compounding these effects is the production of molecular ions formed between analyte atoms/ions with themselves, or with the discharge gas and contaminant species (e.g. $\mathrm{Cu}_{2}{ }^{+}, \mathrm{ArCu}^{+}, \mathrm{FeOH}^{+}, \mathrm{PdH}^{+}, \mathrm{CeO}^{+}$).

In some cases, simply optimizing instrumental parameters ${ }^{4}$ and using pure gas delivery systems ${ }^{5}$ can easily reduce interferences due to contaminant gases. Getters ${ }^{6-8}$ and cryogenic cells or coils ${ }^{9-14}$ can also aid the removal of deleterious water in plasma sources. An alternative approach to reduce spectral complexity before ions enter the mass spectrometer is to couple together two or more atomization/ionization sources. This philosophical approach has been covered in detail by Borer and Hieftje. ${ }^{15}$ It is possible that even after all efforts have been made to reduce spectral interferences in the ion source, persistent isobars will still make their way into the mass spectrometer. In these 
cases, the mass spectrometer system can be used to elucidate the overlapping peaks. The most direct approach is to use higher-resolution instruments such as double-focusing magnetic sectors, ${ }^{16-19}$ ion-cyclotron-resonance mass spectrometers (ICR-MS), ${ }^{20-23}$ or slow-scanning quadrupole ion traps. ${ }^{24,25}$ The former two of which may be too costprohibitive for most practitioners. Mass spectrometry systems utilizing linear quadrupoles can be used in several different manners in order to remove molecular and plasma-gas interferences. Collision-induced dissociation (CID) of diatomic and polyatomic ions in double- ${ }^{26,27}$ and triple-quadrupoles ${ }^{28}$ has been successful in this regard. Ion-molecule reactions, particularly charge exchange reactions, also have proven useful for removing plasma gas species and contaminant gas ions in elemental determinations. ${ }^{11,21,29-31}$ Dynamic reaction cells using reagent gases in linear quadrupoles have also been used to isolate specific ions of interest. ${ }^{25,31}$

Ion-molecule reactions in quadrupole ion traps (QIT) have been used to remove isobaric interferences in atomic mass spectrometry. ${ }^{6,9,11,20,25,32-34} \mathrm{CID}$ in QITs also has been shown to be capable of dissociating strongly bound ions with higher efficiencies than in linear quadrupole systems. ${ }^{20,}{ }^{33-36}$ In one example, it was revealed that the dissociation rate of $\mathrm{TaO}^{+}$was greater than the scattering rate of $\mathrm{Au}^{+}$, at the same nominal mass, when each ion was separately subjected to the same excitation conditions in the trap. This implied, although it was not verified at the time, that diatomic ions could be attenuated in the presence of an atomic ion occurring at the same nominal mass. At the present time, no reports have demonstrated applications for the use of CID in QITs to remove strongly bound diatomic interferences from atomic mass spectra. 
The goal of this dissertation is to understand the fundamental characteristics, and to extend the future possibilities, of two approaches to reducing isobaric interferences in glow discharge mass spectrometry (GD-MS). The ion source considered in this work is the pulsed glow discharge (PGD) source, and this source is known to offer numerous advantages over steady state, or continuous power glow discharges, predominantly because of the time-dependent nature of the discharge. Mass spectrometers used in this work are a linear time-of-flight mass spectrometer (ToF-MS) and a QIT-MS, the latter of which has been shown to offer possibilities for beneficial ion-molecule reactions, including CID.

The first three chapters of this dissertation deal with understanding the elementary processes inherent in the PGD and the possibilities offered for time-gated enhancement of analytical signals with respect to plasma species and molecular interferences. The latter three chapters focus on QIT techniques coupled to the PGD source. CID in the QIT is used first to dissociate molecular ions at the same nominal mass as atomic ions, and later to demonstrate that the dissociation rates of metal oxide ions is mass-dependant. In the course of this study, it was necessary to deliberately form the metal oxides for these analyses. Following this work a method was developed to determine the oxidation rates and reaction rates for various uranium compounds. The observation of certain reactions also leads to some previously unknown thermochemical values.

Because considerably more work has been done with steady state discharges, there is an extensive array of literature on the subject. An understanding of steady-state discharges is useful, and necessary, for understanding pulsed plasmas because pulsed glow discharges often reach a steady state condition before the voltage is terminated. 
Instead of repeating the now-common historical perspective on steady-state glow discharge sources and their applications, the reader will be directed to numerous books, reviews, and tutorials on this issue. The remainder of this introduction will focus on the specific development of pulsed glow discharges, a category of plasmas defined by the gas pressure (0.1-10 Torr) and current density (0.0001-1 $\left.\mathrm{A} \mathrm{cm}^{-3}\right)$ during operation, and the transient nature of the supplied power.

Two books have been devoted to the fundamental characteristics and applications of continuous power glow discharge sources. ${ }^{37,} 38$ These books serve as invaluable references for learning the fundamental processes and applications of glow discharges. The description of glow discharges and additional conceptual enlightenment can be gleaned from various review articles. ${ }^{39-42} \mathrm{~A}$ particularly thorough review of hollow cathode glow discharges - a class of glow discharges with specific electrode geometries-was performed by Caroli. $^{43}$ Glow discharges, and their various accompanying detection methods, have also been compared and contrasted with other plasma sources in numerous review-type articles. ${ }^{44-47}$ In these comparisons, glow discharges are shown to afford numerous advantages over other plasma sources. These advantages include design simplicity and ease of use, spectral simplicity and ease of interpretation, greater compatibility with mass spectrometric systems, sample depth profiling ability for solids, and low consumption of support gas (i.e. argon). The limitations of glow discharges are also highlighted. These include a lack of standard reference materials for quantitative analyses and the difficulty of analyzing liquids and gas samples (although much work is being done in this area, as discussed later). 


\subsection{Pulsed glow discharges: a historical perspective}

Man's first contacts with plasmas, or discharges, probably occurred on the few unfortunate occasions he was struck by lightning. It wouldn't have taken much cognitive ability to realize that such a "pulsed discharge" is rather powerful, and it is not surprising that man spent considerable efforts attempting to harness this power. It took until the discovery of electricity, however, and the development of vacuum pumps, before we

could recreate the phenomena at will. As early as 1822 Sir Humphry Davy noted "The relations of electricity to space, as nearly void of matter as it can be made on the earth's surface, are connected with many important queries bearing upon the nature of heat, light, electricity, and magnetism". He had the foresight that the study of plasmas and discharges would teach us a great deal about the fundamental nature of matter.

In 1858, Julius Plücker showed that by electrifying two electrodes within the vacuum of a glass vessel he could drive a current and cause the glass to glow with a greenish light near the positive electrode. ${ }^{49}$ These experiments were made possible because of the glass-blowing expertise and vacuum pumps developed by Heinrich Geissler. Plücker's student, Wilheim Hittorf, placed solid objects between the two electrodes and found that a shadow was cast on the glass. These observations were verified by a German physicist Eugen Goldstein, who coined the term for the stream of radiation "cathode rays", and by a British physicist, William Crooks, who showed that the "rays" emitted from the negative electrode (the cathode) traveled in straight lines. The English chemist J. J. Thompson continued along these lines and identified that the "cathode rays" had a ne gative charge and he was able to determine their mass to be a 
small fraction of that of the atom. ${ }^{50} \mathrm{He}$ had identified the electron. Thus followed an amazing tale of the discovery of sub-atomic particles and the structure of the atom. ${ }^{51}$

Crookes was fascinated by the light emitted by different elements in his plasmas—especially because German physicist Gustav Kirchhoff had identified that each element emits its own characteristic spectra of lines. ${ }^{49}$ Not only was this theory used to identify a new element (helium) in the sun, but Kirchhoff himself had used this hypothesis to identify several new elements, including cesium (ccesius meaning blue), after its characteristic blue glow, and rubidium (rubidus meaning very dark red). Whereas Kirchhoff heated his elements to incandescence to study their emissions, Crookes developed reliable methods for determining the wavelength and emission intensity of spectra emitted by different elements in his plasmas. In this way he identified the new element thallium by its brilliant green emission. In 1895 he collected the emission spectra of the newly discovered argon, ${ }^{52}$ generously given to him by the discoverers Lord Rayleigh and William Ramsay. ${ }^{53}$

In these first spectra of argon, Crookes performed a simple but particularly enlightening experiment. He adjusted the pressure and voltage of the discharge. On lowering the pressure he discovered that the emission spectra turned from a red to a rich steel blue color. The visible difference was dramatic—so much so that this led him to suggest that argon might actually consist of two elements! $!^{54}$ In retrospect, we know that the differences in the spectra that Crookes observed were due to the plasma stability, and the associated probabilities of populating certain electron energy levels under the different conditions. At high pressures, a stable plasma was obtained and most of the emissions originated from lower lying energy levels. At low pressures, his plasma was 
unstable and the current passed in transient flashes, hence, his plasma was pulsed (albeit irregularly). In the pulsed plasma, argon ions would recombine with electrons at the end of the voltage pulse to populate the high-lying energy levels. This caused a population inversion and a very different looking emission spectrum. Of course, some lines were observed in both spectra.

In the course of these experiments Crookes also noted that platinum from the electrodes "spatters" over the glass bulbs in a process he called "electrical evaporation". 52 We now call this process "sputtering" and it is the main method that analytical chemists use to deliberately introduce the cathode material into the plasma for chemical analyses. Once in the plasma, atoms that have been sputtered from the surface are subjected to various kinds of excitation and ionization events, and it is up to the analytical chemist to somehow measure the sputtered atoms and determine the nature of the sample from which the atoms originated. Depending on the nature of the measurement, the analytical chemist will adjust the plasma conditions to optimize the signal being studied.

In 1886, Goldstein noticed luminous streams arising from rays traveling in the opposite direction to cathode rays that had passed through some perforations in the cathode. ${ }^{55}$ They were later confirmed to possess positive charge and so Thompson called them "positive rays". ${ }^{2}$ Under the guidance of J. J. Thompson, F. W. Aston used the positive rays of neon to determine that neon actually consisted of two differentlyweighing atoms, in different proportions, that he called isotopes. The isotopes weighed 20 and 21 mass units and were present in such a ratio that the weighted average agreed remarkably well with the known atomic weight of 20.18 . He went on to characterize the nature of isotopes for which he received the Nobel Prize in 1922. In the same year, Aston 
published Isotopes ${ }^{56}$ and this, along with his Nobel Prize, helped him gain more exposure and generate more interest in the determination of isotope ratios of the elements.

From the same group at Cavendish Laboratories in Cambridge (England), J. W. Bispham was able to determine the electrical fields in the plasma by using an "exploring electrode, ${ }^{, 57}$ (nowadays referred to as a Langmuir probe). By varying the capacitance and resistance of the external electric circuit of the discharge Bispham showed that he could produce a regularly pulsed glow discharge with repetition frequencies as long as one second and so quick as to be beyond audible. He characterized the emissions of a helium pulsed glow discharge under different conditions and noted that at low repetition frequencies "the discharge passed by a brilliant flash of such intensity as to cause anxiety for the safety of the apparatus". ${ }^{57} \mathrm{He}$ demonstrated that altering the electronic circuit of the power supply could vary the emission intensity of the gas.

By the early part of the 1900's, glow discharges had been shown to be extremely useful for generating emission spectra and mass spectra of atoms-an observation that is still being exploited to this day. The work that has been done since these early beginnings has focused on ways to improve such measurements, and on how to interpret those results. One method that has been recently exploited for analytical advantages is the method of pulsing, or modulating, the voltage that supports the glow discharge plasma. In so doing, the plasma undergoes various levels of excitation at different stages in the pulse cycle, and this results in temporal domains that are better or worse suited to certain measurements.

In the 1920's pulsed glow discharges were used to study fundamental properties of plasmas such as the recombination rates of electrons and ions. ${ }^{58-60}$ In all cases, 
emissions from higher energy levels were observed in the afterglow regime, relative to the during-pulse emissions. Kenty ${ }^{58}$ and Hayner ${ }^{59}$ independently showed that it took approximately $25 \mu$ s before the electrons would start to recombine once the voltage had been removed. Hayner ventured that this must be the time required for the electrons to cool down, via collisions with the bath gas, before they could recombine with argon ions. ${ }^{59}$ Once the electrons had cooled down (thermalized) and recombined with an argon ion, they were temporarily trapped in a highly excited state. Decomposition of these electrons to more stable lower-lying energy states was accompanied by the emission of characteristic wavelengths of light. Thus, the plasma emission intensity actually increased soon after the voltage had been removed. This is the reason for the term "afterglow" that is so commonly used to describe the time period following voltage termination.

Kenty also notes some other interesting observations: ${ }^{58}$

1) The afterglow emission intensity increased as the argon purity increased, indicating the deleterious effects of gas impurities.

2) The occurrence of sodium vapors in his discharges that he had introduced "quite accidentally" were the strongest lines emitted by the plasma. Emissions from the D lines were observed while the voltage was on, but not in the afterglow. This demonstrated that electrons do not have sufficient energy in the afterglow to excite atoms to low-lying energy states. The occurrence of sodium emission from higher lying states was not described in his report.

3) Additionally-applied after-voltages could be used to prolong the dark period (and therefore the onset of the afterglow) and enhance or decrease the eventual afterglow intensity. He demonstrated that by keeping the electrons at above thermal kinetic 
energies they were unlikely to recombine_-so the afterglow could not occur-but that the electrons would still be available to recombine when the voltage was finally removed.

4) The presence of $<1 \% \mathrm{H}_{2}$ admitted to the argon did not affect the afterglow emissions. He suspected that the $\mathrm{H}_{2}$ would have quenched any metastable argon atoms that were present. The fact that the added hydrogen had no observable effect led him to conclude that metastables played no part in the afterglow.

Pulsed GDs were later used to generate ions and excited neutral species for subsequent analysis by flow tubes. ${ }^{61-66}$ These devices were commonly known as flowing afterglow techniques. Flow tubes were useful for determining the rates of chemical reactions of glow-discharge-generated ions because the flow rates of reagents, and hence the timeframe for the reaction, could be varied. The field of gas-phase kinetics therefore often employed these apparatus for fundamental studies.

An interesting discovery made by Biondi in the mid 1900's was that the electron density, and thus the degree of ionization, actually increased immediately following the removal of the pulsed voltage. ${ }^{63}$ This observation seemed in contrast to the evidence provided by Hayner and Kenty—-that electrons recombined after $\sim 25 \mu \mathrm{s}$ — but was found to be consistent when one experimental variable was altered...the pulse width. Biondi showed that if the voltage-pulse width was shorter than $200 \mu$ s, then recombination would occur on the timescales similar to Kenty's. At pulse widths longer than $200 \mu$ s, the electron density in the afterglow would first increase before decreasing. This observation was linked to the presence of metastable argon atoms that were present at the end of the voltage pulse. ${ }^{65}$ Metastable atoms are trapped at very high energies $(11.55 \mathrm{eV})$ and have 
sufficient energy to form an argon ion and an electron upon colliding with one another $(\mathrm{IE}(\mathrm{Ar})=15.759 \mathrm{eV})$. Thus, if the pulse width was sufficiently long enough to form a substantial population of metastable atoms, there would be a higher probability that they would collide with one another in the afterglow to cause increased ionization. Because Kenty's pulse width was shorter than $200 \mu$ s, this explains why he observed that metastables did not play a role in the afterglow process.

It was also well known that if any atom or molecule present in the afterglow had an ionization potential below that of the metastable states of the support gas (argon, at $11.55 \mathrm{eV}$, in these examples), then these species would be readily ionized by the metastables, a process known as Penning ionization. ${ }^{67}$ An example was given by Knewstubb and Tickner, ${ }^{68}$ in which they report that the ionization rate for Xe atoms in an argon plasma was $\sim 2000$ times greater than expected. It would be several decades, however, before these observations were repeated and exploited for analytical purposesthe development of which is covered in the next section. 


\subsection{Analytical pulsed glow discharges for optical spectroscopy}

In 1967 Dawson and Ellis published one of the first applications of a pulsed glow discharge source for an analytical analysis. ${ }^{69}$ Using square wave pulses (with pulse widths of $15-40 \mu \mathrm{s})$ on top of a steady-state discharge of various hollow cathode lamps they observed a 50-500 fold increase in emission intensity of the cathode elements. The hollow cathodes were used as light sources for flame atomic absorbance determinations of different elements in solutions. The increase in intensity of the hollow cathode sources, caused by the pulsing, enabled a substantial decrease in the limits of detection and greater sensitivity to be achieved than conventional dc operation of the lamps. Also, because the cathodes were not continually being sputtered at high operating voltages, the working life of the lamps were greatly extended. It was even noted that lamps that had previously been taken out of service because they could no longer excite resonance radiation (in the constant dc mode) were found to operate perfectly satisfactorily in the pulsed mode. There then followed a surge of interest in using pulsed glow discharges as sources for absorption and fluorescence measurements. ${ }^{70-73}$

Cordos and Malmstadt ${ }^{74}$ later characterized the output radiance of hollow cathode lamps operated in the pulsed mode and found them to be 40-200 times greater than the maximum in the dc mode. They also emphasize that the working life of the lamps can be vastly extended using this approach. Piepmeier and Galan ${ }^{75}$ used a very accurate interferometer to measure the line profiles emitted by $\mathrm{Cu}$ and $\mathrm{Ca}$ atoms in pulsed hollow cathodes and found that the sputtered atoms diffuse or are convectively ejected from the cathode surface at a rate of $\sim 10000 \mathrm{~cm} \mathrm{~s}^{-1}$ during the first $20 \mu$ s of pulse operation. Once the sputtered species filled the gas volume the diffusion gradient and temperature 
gradients were smaller and the velocity of the sputtered species in the direction away from the cathode surface decreased (i.e. resembled steady-state conditions).

Butler and coworkers ${ }^{76-79}$ promulgated the use of pulsed glow discharges as spectral line isolators. Time-resolved spectroscopy revealed that considerable emission intensity was being emitted after the pulsed voltage had been removed. Strauss et al. showed that the emission was related to the post-pulse increase in metastable argon atoms and that this increase was probably related to recombination of argon ions with thermal electrons. ${ }^{80}$ By adding methane to the bath gas to quench the metastables, they were able to reduce the number of argon metastables and sputtered atom emissions in the afterglow. In the absence of mass spectral evidence, they proposed that the metastable atoms did not Penning ionize the sputtered atoms. Later evidence would show that a great deal of sputtered atoms are ionized in the afterglow, but that they are formed in the ground state and so they are not observable by emission spectroscopy. ${ }^{81,82}$

The atomic fluorimeter of Butler and coworkers was described and reviewed by Ferreira et al. ${ }^{83}$ and included optical measurements of the argon metastable atoms and sputtered analyte atoms. Ferreira et al. show that the ground state copper atom density remains virtually unchanged for at least $700 \mu \mathrm{s}$ after the voltage was terminated. ${ }^{83}$ At this delay time $(700 \mu \mathrm{s})$, emissions from the argon bath gas and from excited copper atoms were considerably smaller than while the voltage was applied. This implied that the pulsed glow discharge might be a very simple method for obtaining a large population of ground state sputtered atoms in the absence of background emissions-the ideal environment for low-interference absorbance or fluorescence measurements. ${ }^{84}$ Winefordner and coworkers confirmed this promise $\mathrm{e}^{85-87}$ and demonstrated that solution 
residues of lead on a graphite cathode could be quantified over a range of 6 orders of magnitude. Winefordner and coworkers were also able to obtain limits of detection of $\sim 20$ pg by using the pulsed glow discharge to generate a reservoir of atoms for laser induced fluorescence measurements. ${ }^{85}$ Pan and King also exploited the atom-reservoirnature of the pulsed glow discharge by determining trace elements of metals in graphite matrices by atomic absorption spectrometry. ${ }^{88}$

One of the most recent applications for pulsed glow discharge optical spectroscopy has been for the depth profiling of thin-film-solids. ${ }^{89-91}$ Steady-state glow discharges had been used for depth profiling for some time, ${ }^{16,92-95}$ but the pulsed system offered numerous advantages. Pulsing the plasma at higher voltages enhances the sputtering rate and signal intensities by 1-4 orders of magnitude, thus allowing minor

constituent elements to be determined in the different layers. ${ }^{89}$ Also, the pulse width and voltage can be altered to affect the sputtering rate and this enables resolution for the analysis of films as thin as $10 \mathrm{~nm}$ to be realized in as little as 10 seconds. $^{89}$

\subsection{Analytical pulsed glow discharges for mass spectrometry}

In the early 1990s Klingler, Harrison and coworkers first demonstrated the analytical application of pulsed glow discharges for mass spectral analysis. ${ }^{96,97}$ Although analytical glow discharge mass spectrometers had been in use for some time, ${ }^{98,}{ }^{99}$ the benefits of modulating the glow discharge voltage had not been realized until the last decade. Klingler et al. showed that atoms derived from the sputtered cathode materials were preferentially ionized in the afterglow time period, whereas the bath gas (argon) ions were significantly reduced in the afterglow. ${ }^{96}$ Therefore, by using a time gated 
quadrupole mass spectrometer they could not only selectively monitor the analyte ions in the absence of spectral interferences, but the analyte signals were actually enhanced in this time domain. They also studied the effect of the voltage pulse shape on the signal responses and found that a fast decrease in voltage, in excess of $400 \mathrm{~V}$, at the end of the pulse was necessary to achieve significant post-pulse ionization of the analyte ions. They related this effect to the post-pulse formation of metastable argon atoms, which they cite as being responsible for ionizing the analyte atoms in the afterglow. Changing the support gas to neon and noting that the neon metastables were then able to ionize water molecules in the afterglow supported this postulate.

Harrison's group then went on to perform considerable research in this area. ${ }^{100-105}$ One of the inherent advantages of pulsed glow discharges is that while the voltage is off, no current flows and the cathode material is able to cool. Because of the cooling during the 'off' period, higher voltages (and currents) can be applied during the 'on' period than is normally permissible in steady state discharges. Not only are the sputtering rates higher in the pulsed mode, but also the excitation and ionization rates are also greater in the pulsed mode. $^{106}$

The King group has been active in determining the mechanisms of post-pulse ionization in pulsed glow discharges. ${ }^{81,82,88,107-109}$ It was shown that the addition of methane to the argon carrier gas greatly decreased the number of analyte ions formed in the afterglow. ${ }^{81,82,107}$ As with previous quenching experiments with methane, ${ }^{80,110,111}$ this effect was linked to the destruction of metastable argon atoms that would otherwise Penning ionize the analyte atoms. It was also discovered that the post-pulse increase in ground state analyte ions can be larger than the post-pulse increase in analyte atom 
emissions. ${ }^{107}$ Therefore, analyte ionization is more enhanced than analyte excitation in the afterglow. Using a triple quadrupole mass spectrometer fitted with a kinetic energy discriminator (Bessel box) they were also able to determine that the ions analyzed in the afterpeak have considerably more kinetic energy than ions formed during the voltage on period. $^{81,82}$ This effect was thought to be related to the net positive plasma potential that ensued in the afterglow because of the difference in diffusion rates for electrons and ions. This plasma potential was later verified by another group ${ }^{112}$ and poses as another interesting method to discriminate against interfering ions in mass spectral analyses.

One of the advantages of pulsed glow discharges mentioned above is the ability to enhance the sputtered atom ionization in the afterglow relative to the plasma gas and impurity gas ions. This selectivity has been illustrated for the determination of ${ }^{40} \mathrm{Ca}^{+}$in the presence of ${ }^{40} \mathrm{Ar}^{+} .{ }^{113}$ In these examples the quantitation of calcium is made at some time in the afterglow period when the plasma gas ions $\left(\mathrm{Ar}^{+}\right)$have diminished to near zero signal, while the calcium signals are much larger than steady state dc conditions. A similar approach has also been realized for quadrupole ion traps using a PGD source. ${ }^{114}$

Other recent applications of pulsed glow discharges have focused on the ionization and fragmentation of organic molecules at different stages of the plasma. ${ }^{115,116}$ Majidi and coworkers showed that the harsh conditions of electron excitation while the voltage is on fragments the molecules, giving structural information, while the relatively soft method of Penning ionization in the afterglow provides molecular weight information of the organics. ${ }^{115,116}$ Therefore, in a single source, one is able to obtain nearly concurrent molecular weight and fragmentation information for organic molecules. This approach is highly desirable for gas chromatography detection where identification 
of unkown eluents is often necessary. The Hieftje group has recently been developing a switched dc glow discharge for this purpose with promising success. ${ }^{17-119}$ Peak shapes were reasonably well preserved, indicating that the dead-volume of the detector was sufficiently small, and detection limits and quantitative ability were very respectable.

In summary, it is generally accepted that pulsing the analytical glow discharge, with dc or rf voltages, enhances the sputtering, ionization and excitation of the cathode materials. Pulsed dc glow discharges can be applied to conducting and poorly conducting solids whereas pulsed (or steady state) rf voltages can be used for non-conducting materials. Various groups have shown that adding methane to the pulsed glow discharge has the effect of decreasing the number density of metastable argon atoms in the afterglow, and this results in less ionization/excitation of the analyte atoms. For analytical purposes, it is therefore necessary to keep the plasma as free of molecular impurities as possible. The largest benefit to be gained from pulsed glow discharges is in time-gated detection methods that can allow one to observe analyte emission or ion signals at a time when other plasma species are greatly reduced. The QIT has been shown to benefit from such time-gated detection, but spectral interferences from strongly-bound diatomics are not completely removed by this method. Therefore, additional approaches are required to eliminate as completely as possible, the interfering ions from the analytical ions. 


\subsection{References}

(1) Roboz, J. Introduction to Mass Spectrometry: Instrumentation and Techniques; John Wiley \& Sons, Inc.: New York, 1968.

(2) Thompson, J. J. Rays of Positive Electricity and their Application to Chemical Analyses; Longmans, Green: New York, 1913.

(3) Knight, D. M. Atoms and Elements; Hutchinson: London, 1967.

(4) Oksenoid, K. G.; Liebich, V.; Pietzsch, G. Fresenius'. J. Anal. Chem. 1996, 355, 863-865.

(5) Valiga, R. E.; Duckworth, D. C.; Smith, D. H. Rapid Commun. Mass Spectrom. 1996, $10,305-310$.

(6) Barinaga, C. J.; Eiden, G. C.; Alexander, M. L.; Koppenaal, D. W. Fresnius' J. Anal. Chem. 1996, 355, 487-493.

(7) Mei, Y.; Harrison, W. W. Spectrochim. Acta, Part B 1991, 46, 175-182.

(8) Ratliff, P. H.; Harrison, W. W. Appl. Spectrosc. 1995, 49, 863-871.

(9) Barinaga, C. J.; Kopenaal, D. W. Rapid Commun. Mass Spectrom. 1994, 8, 71-76.

(10) DeGendt, S.; VanGrieken, R. E.; Ohorondnik, S. K.; Harrison, W. W. Anal. Chem. 1995, 67, 1026-1033.

(11) Koppenaal, D. W.; Barinaga, C. J.; Smith, M. R. J. Anal. At. Spectrom. 1994, 9, 1053-1058.

(12) Ohorodnik, S. K.; Harrison, W. W. Anal. Chem. 1993, 65, 2542-2544.

(13) Ohorodnik, S. K.; Harrison, W. W. J. Anal. At. Spectrom. 1994, 9, 991-996.

(14) Ratliff, P. H.; Harrison, W. W. Spectrochim. Acta, Part B 1994, 49, 1747-1757. 
(15) Borer, M. W.; Hieftje, G. M. Spectrochim. Acta Rev 1991, 14, 463-486.

(16) Stuewer, D. Fresnius' J. Anal. Chem. 1990, 337, 737-742.

(17) Donahue, D. L.; Petek, M. Anal. Chem. 1991, 63, 740-744.

(18) Wayne, D. M.; Yoshida, T. M.; Vance, D. E. J. Anal. At. Spectrom. 1996, 11, 861-869.

(19) Jacubowski, N.; Feldmann, I.; Steuwer, D. J. Anal. At. Spectrom. 1997, 12, 151157.

(20) Duckworth, D. C.; Eyler, J. R.; Watson, C. H. In Inorganic Mass Spectrometry.; Barshick, C. M., Duckworth, D. C., Smith, D. H., Eds.; Marcel Dekker, Inc.: New York, 2000, pp 329.

(21) Douglas, D. J. Can. J. Spectrosc. 1989, 34, 38-49.

(22) Barshick, C. M.; Goodner, K. L.; Watson, C. H.; Eyler, J. R. Int. J. Mass Spectrom. 1998, 178, 73-79.

(23) Watson, C. H.; Barshick, C. M.; Wronka, J.; Laukien, F. H.; Eyler, J. R. Anal. Chem. 1996, 68, 573-575.

(24) Olesik, J. W. In Inorganic Mass Spectrometry; Barshick, C. M., Duckworth, D. C., Smith, D. H., Eds.; Mercel Dekker, Inc: New York, 2000.

(25) Eiden, G. C.; Barinaga, C. J.; Koppenaal, D. W. J. Anal. At. Spectrom. 1999, 14, 1129-1132.

(26) Duckworth, D. C.; Marcus, R. K. Appl. Spectrosc. 1990, 44, 649-655.

(27) Mei, Y.; Duckworth, D. C.; Cable, P. R.; Marcus, R. K. J. Am. Soc. Mass Spectrom. 1994, 5, 845-851. 
(28) King, F. L.; Harrison, W. W. Int. J. Mass Spectrom. Ion. Processes 1989, 89, 171185.

(29) Rowan, J. T.; Houk, R. S. Appl. Spectrosc. 1989, 43, 976-980.

(30) Eiden, G. C.; Barinaga, C. J.; Koppenaal, D. W. J. Anal. At. Spectrom. 1996, 11, 317-322.

(31) Eiden, G. C.; Barinaga, C. J.; Koppenaal, D. W. Rapid Commun. Mass Spectrom. 1997, 11, 37-42.

(32) Eiden, G. C.; Barinaga, C. J.; Koppenaal, D. W. J. Am. Soc. Mass Spectrom. 1996, $7,1161-1171$.

(33) Duckworth, D. C.; Barshick, C. M. Anal. Chem. 1998, 70, 709A-717A.

(34) McLuckey, S. A.; Glish, G. L.; Duckworth, D. C.; Marcus, R. K. Anal. Chem. 1992, 64, 1606-1609.

(35) Duckworth, D. C.; McLuckey, S. A., 26th Federation of Analytical Chemistry and Spectroscopy Societies Conference, Vancouver, Canada, October 1999.

(36) Duckworth, D. C.; Goeringer, D. E.; McLuckey, S. A. J. Am. Soc. Mass Spectrom. 2000, 11, 1072-1078.

(37) Chapman, B. Glow Discharge Processes; John Wiley and Sons: New York, 1980.

(38) Marcus, R. K. Glow Discharge Spectroscopies, 1 ed.; Plenum Press, 1993.

(39) Bogaerts, A.; Gijbels, R. Spectrochim. Acta, Part B 1998, 53, 1-42.

(40) Harrison, W. W.; Hess, K. R.; Markus, R. K.; King, F. L. Anal. Chem. 1986, 58, 341A-356A.

(41) Harrison, W. W.; Barshick, C. M.; Klingler, J. A.; Ratliff, P. H.; Mei, Y. Anal. Chem. 1990, 62, 943A-949A. 
(42) Broekaert, J. A. C. J. Anal. At. Spectrom. 1987, 2, 537-542.

(43) Caroli, S. Prog. Analyt. Atom. Spectrosc. 1983, 6, 253-292.

(44) Winefordner, J. D.; II, E. P. W.; Smith, B. W. J. Anal. At. Spectrom. 1996, 11, 689-702.

(45) Marcus, R. K.; Evans, E. H.; Caruso, J. A. J. Anal. At. Spectrom. 2000, 15, 1-5.

(46) Baude, S.; Broekaert, J. A. C.; Delfosse, D.; Jakubowski, N.; Fuechtjohann, L.;

Orellana-Velado, N. G.; Pereiro, R.; Sanz-Medel, A. J. Anal. At. Spectrom. 2000, $15,1516-1525$.

(47) Colodner, D.; Salters, V.; Duckworth, D. C. Anal. Chem. 1994, 66, 1079-1089.

(48) Davy, H. Proc. R. Soc. London 1822, 159.

(49) Hellemans, A.; Bunch, B. The Timetables of Science; Touchstone: New York, 1991.

(50) Thompson, J. J. Phil. Mag. 1897, fifth series, 302-311.

(51) Pullman, B. The Atom in the History of Human Thought; Oxford University Press: New York, 1998.

(52) Crookes, W. Philos. Trans. R. Soc. London 1895, 186, 243-252.

(53) Rayleigh, L.; Ramsay, W. Philos. Trans. R. Soc. London 1895, 186, 187-242.

(54) At the time, this suggestion would have seemed not at all unreasonable: similar observations had just been made for nitrogen. Nitrogen, of course, exists in diatomic form and its emission characterisitcs in this state are very different from atomic nitrogen.

(55) Goldstein, E. Berl. Ber. 1886, 39, 691-.

(56) Aston, F. W. Isotopes, 1 ed.; Edward Arnold: London, 1922. 
(57) Bispham, J. W. Proc. R. Soc. London A 1908, 8, 477-487.

(58) Kenty, C. Phys. Rev. 1928, 32, 624-635.

(59) Hayner Zeits f. Physik 1925, 35, 365.

(60) Mohler Phys. Rev. 1928, 31, 187.

(61) Biondi, M. A.; Brown, S. C. Phys. Rev. 1949, 76, 1697-1700.

(62) Biondi, M. A. Phys. Rev. 1951, 83, 1078-1080.

(63) Biondi, M. A. Phys. Rev. 1951, 82, 453-454.

(64) Biondi, M. A.; Holstein, T. Phys. Rev. 1951, 82, 962-963.

(65) Biondi, M. A. Phys. Rev. 1952, 88, 660-665.

(66) Biondi, M. A.; Chanin, L. M. Phys. Rev. 1954, 94, 910-916.

(67) Penning, F. M. Physik 1925, 46, 225.

(68) Knewstubb, P. F.; Tickner, A. W. J. Chem. Phys. 1962, 36, 684-693.

(69) Dawson, J. B.; Ellis, D. J. Spectrochim. Acta, Part A 1966, 23, 565-590.

(70) Omenetto, N.; Fraser, L. M.; Winefordner, J. D. Appl. Spectrosc. Rev. 1973, 7, 147.

(71) DeJong, G. J.; Piepmeier, E. H. Anal. Chem. 1974, 46, 318.

(72) Osten, D. E.; Piepme ier, E. H. Appl. Spectrosc. 1973, 27, 165.

(73) Prugger, H.; Grosskopf, R.; Torge, R. Spectrochim. Acta, Part B 1971, 26, 191.

(74) Cordos, E.; Malmstadt, H. V. Anal. Chem. 1973, 45, 27-32.

(75) Piepmeier, E. H.; deGalan, L. Spectrochim. Acta, Part B 1975, 30, 263-279.

(76) Butler, L. R. P.; Kroger, K.; West, C. D. Spectrochim. Acta, Part B 1975, 30, 489499.

(77) Butler, L. R. P.; Kroger, K.; West, C. D. Spectrochim. Acta, Part B 1975, 30, 489. 
(78) Human, H. G. C.; Ferreira, N. P.; Kruger, R. A.; Butler, L. R. P. Analyst 1978, $103,469$.

(79) Human, H. G. C.; Strauss, J. A.; Butler, L. R. P. Spectrochim. Acta, Part B 1980, $35,207$.

(80) Strauss, J. A.; Ferreira, N. P.; Human, H. G. C. Spectrochim. Acta, Part B 1982, 37, 947-954.

(81) Pan, C.; King, F. L. J. Am. Soc. Mass Spectrom. 1993, 4, 727-732.

(82) Pan, C.; King, F. L. Anal. Chem. 1993, 65, 3187-3193.

(83) Ferreira, N. P.; Strauss, J. A.; Human, H. G. C. Spectrochim. Acta, Part B 1983, 34, 899-911.

(84) Chakrabarti, C. L.; Headrick, K. L.; Hutton, J. C.; Bicheng, Z.; Bertels, P. C.; Back, M. H. Anal. Chem. 1990, 62, 574-586.

(85) Dijk, C. V.; Smith, B. W.; Winefordner, J. D. Spectrochim. Acta. 1982, 37B, 759768.

(86) Smith, B. W.; Omenetto, N.; Winefordner, J. D. Spectrochim. Acta, Part B 1984, 39, 1389-1393.

(87) Womack, J. B.; Gessler, E. M.; Winefordner, J. D. Spectrochim. Acta, Part B 1991, 46, 301-308.

(88) Pan, C.; King, F. L. Appl. Spectrosc. 1993, 47, 300-304.

(89) Yang, C.; Ingeneri, K.; Mohill, M.; Harrison, W. W. Anal. Chem. 1999, 71, 53285334.

(90) Oxley, E.; Yang, C.; Harrison, W. W. J. Anal. At. Spectrom. 2000, 15, 1241-1245. 
(91) Su, Y.; Yang, P.; Zhou, Z.; Wang, X.; Li, F.; Huang, B.; Ren, J.; Chen, M.; Ma, H.; Zhang, G. Spectrochim. Acta, Part B 1997, 53, 1413-1420.

(92) Greene, J. E.; Whelan, J. M. J. Appl. Phys. 1973, 44, 2509-2513.

(93) Belle, C. J.; Johnson, J. D. Appl. Spectrosc. 1973, 27, 118-124.

(94) Bengston, A. Spectrochim. Acta, Part B 1994, 49, 411-429.

(95) Weiss, Z. J. Anal. At. Spectrom. 1995, 10, 891-895.

(96) Klinger, J. A.; Barshick, C. M.; Harrison, W. W. Anal. Chem. 1991, 63, 25712576.

(97) Klinger, J. A.; Savickas, P. J.; Harrison, W. W. J. Am. Soc. Mass Spectrom. 1990, 1.

(98) King, F. L.; Harrison, W. W. Mass Spec. Rev. 1990, 9, 285-213.

(99) King, F. L.; Teng, J.; Steiner, R. E. J. Mass Spec. 1995, 30, 1061-1075.

(100) Hang, W.; Walden, W. O.; Harrison, W. W. Anal. Chem. 1996, 68, 1148-1152.

(101) Harrison, W. W.; Hang, W. Fres. J. Anal. Chem. 1996.

(102) Harrison, W. W.; Hang, W. J. Anal. At. Spectrom. 1996, 11, 835-840.

(103) Pollmann, D.; Ingeneri, K.; Harrison, W. W. J. Anal. At. Spectrom. 1996, 11, 849853.

(104) Hang, W.; Harrison, W. W. Anal. Chem. 1997, 69, 4957-4963.

(105) Hang, W.; Baker, C.; Smith, B. W.; Winefordner, J. D.; Harrison, W. W. J. Anal. At. Spectrom. 1997, 12, 143-149.

(106) Yan, X.; Ingeneri, K.; Hang, W.; Harrison, W. W. J. Anal. At. Spectrom. 2001, $16,819-824$.

(107) King, F. L.; Pan, C. Anal. Chem. 1993, 65, 735-739. 
(108) Pan, C.; King, F. L. Appl. Spectrosc. 1993, 47, 2096-2101.

(109) Steiner, R. E.; Lewis, C. L.; King, F. L. Anal. Chem. 1997, 69, 1715-1721.

(110) Smith, R. L.; Serxner, D.; Hess, K. R. Anal. Chem. 1989, 61, 1103-1108.

(111) Levy, M. K.; Serxner, D.; Angstadt, A. D.; Smith, R. L.; Hess, K. R. Spectrochim. Acta, Part B 1991, 46, 253-267.

(112) Budtz-Jorgensen, C. V.; Bottiger, J.; Kringhoj, P. Surf. Coat. Technol. 2001, 137, 104-110.

(113) Lewis, C. L.; Oxley, E. S.; Pan, C. K.; Steiner, R. E.; King, F. L. Anal. Chem. 1999, 71, 230-234.

(114) Duckworth, D. C.; Smith, D. H.; McLuckey, S. A. J. Anal. At. Spectrom. 1997, $12,43-48$.

(115) Steiner, R. E.; Lewis, C. L.; Majidi, V. J. Anal. At. Spectrom. 1999, 14, 15371541.

(116) Majidi, V.; Moser, M.; Lewis, C.; Hang, W.; King, F. L. J. Anal. At. Spectrom. 2000, 15, 19-25.

(117) Guzowski, J. P.; Hieftje, G. M. Anal. Chem. 2000, 72, 3812-3820.

(118) Guzowski, J. P.; Hieftje, G. M. J. Anal. At. Spectrom. 2000, 15, 27-36.

(119) Guzowski, J. P.; Brockaert, J. A. C.; Ray, S. J.; Hieftje, G. M. J. Anal. At. Spectrom. 1999, 14, 1121-1127. 


\section{Chapter 2}

\section{Metastable Argon Atoms in the Pulsed Glow Discharge}

\subsection{Introduction}

The analytical glow discharge (GD) is a valuable tool for the direct analysis of conducting and non-conducting solids. ${ }^{1-3}$ It is essential to understand the mechanisms of excitation and ionization in the GD in order to optimize the plasma's analytical performance. Extensive studies and modeling of excitation and ionization mechanisms appear in the literature for steady state dc-powered glow discharges. ${ }^{2,}{ }^{4-7}$ There is markedly less information in the literature regarding these mechanisms for rf-powered and pulsed glow discharges even though these two adaptations of the glow discharge appear to hold the greatest promise for analytical applications. ${ }^{8}$

The pulsed, or modulated, application of discharge power produces higher instantaneous concentrations of analyte ions and greater instantaneous emissions than possible with steady state dc current. ${ }^{9-13}$ In such operation, the increased instantaneous power applied during the "on" pulse improves sputter atomization and excitation/ionization; whereas, the "off" period allows the cathode to cool and avoid overheating.

Duncen, et. $a l{ }^{14}$ show that interferences from residual gases reach a minimum during the afterglow time period of these plasmas. The resulting decrease in background signals coupled with high atomic ground state populations affords improved detection

limits for atomic absorption ${ }^{9,15,16}$ and atomic fluorescence. ${ }^{15,17-19}$ Other research results 
demonstrate an enhancement in emission from the analyte species in the afterpeak. ${ }^{12,20-22}$ Observations made perpendicular to the axis defined by the insertion probe afford an additional analytical advantage over 'end-on' measurements because spatial variations of plasma processes can be exploited. ${ }^{19,23,24}$

The mechanisms responsible for afterpeak emission by the sputtered analyte species are more complex than a capture-cascade model would predict. ${ }^{25,26}$ For instance, emission often occurs for a time that is several orders of magnitude longer than the lifetimes of the excited states. This observation strongly suggests a role for long-lived species such as metastable argon atoms. Indeed, Strauss et al. ${ }^{20}$ demonstrate that metastable argon atoms play a significant role in the excitation of copper atoms in the afterpeak (at $\sim 1.8$ Torr, $40 \mathrm{~mA}, 400 \mathrm{~V}$ ). Although the exact mechanism of energy transfer is uncertain, in the absence of $\mathrm{Cu}$ II emissions, they rule out the possibility of Penning ionization. This analysis overlooks the fact that the residual energy from Penning ionization is insufficient to populate excited states of the copper ion, thus the lack of emission alone cannot rule out the role of Penning ionization. A possible mechanism would be Penning ionization of sputtered analyte atoms followed by electron-ion recombination, leading to radiative relaxation.

In fact, mass spectrometric sampling of these plasmas demonstrates that analyte ion formation is enhanced after power termination. ${ }^{21,27-29}$ It appears that these analyte ions form in the afterpeak via Penning ionization. Observations that 1) the argon ion concentration diminishes before the analyte ion afterpeak maximizes, and 2) analyte ion densities exceed those of argon ions support this conclusion. ${ }^{30}$ The addition of methane to the discharge gas $^{31,32}$ to quench metastable argon atoms also inhibits ionization of 
sputtered species in the afterpeak, again strongly implying a role for Penning ionization. $^{27,} 28$ Following along this line, Majidi and coworkers observe distinct differences in ionization mechanisms between the steady state conditions and the afterpeak when they introduce organic vapors into pulsed glow discharges. ${ }^{33,34}$ In those investigations they observe different fragmentation patterns for organics during different time periods in the pulse cycle. The fragmentation patterns observed in the afterpeak strongly suggest reactions with species having energy equivalent to that of metastable argon atoms.

In steady state GD's, it has long been considered that metastable discharge gas atoms play an important role in the ionization of sputtered species. ${ }^{35}$ Hess and coworkers $^{36,37}$ show that Penning ionization is responsible for $40-80 \%$ of the ionization of sputtered species in argon glow discharges operating at 0.5-1.2 Torr, 1-5 mA, and 5003500 V. If Penning ionization accounts for $40-80 \%$ of ionization during voltage application, one might expect that this percentage would be larger in the afterpeak time regime where fewer competing ionization mechanisms are possible.

Because metastable argon atoms are so important in the pulsed GD plasma, this paper focuses on the investigation of these species. In this study laser induced atomic fluorescence (LIF), atomic emission, and atomic absorption measurements provide insight into the formation and disappearance of the two metastable argon atom states $\left({ }^{3} \mathrm{P}_{2}\right.$ state at $11.55 \mathrm{eV}$ and ${ }^{3} \mathrm{P}_{0}$ state at $11.72 \mathrm{eV}$ ). This study should provide a better understanding of the spatial and temporal dynamics occurring within the discharge gas. Our investigations of other species such as analyte atoms/ions, argon ion species, and non-metastable excited state argon atoms are ongoing and will appear separately. 


\subsection{Experimental}

Figure 1a shows the optical setup used for emission and absorption experiments. A stainless steel six-way cross (MDC, MFG Inc., Hayward, CA) with Suprasil optical view ports (Heraeus Quartz, Duluth, GA) housed the glow discharge source. The $4 \mathrm{~mm}$ diameter copper disk samples (unalloyed copper NIST SRM 495, Gaithersburg, MD) were introduced via a $1.27 \mathrm{~cm}$ ball valve using a direct insertion probe. Ultra pure Argon (Airgas, Radnor, PA) at 0.8 Torr was used as the discharge gas. Supporting the discharge was a fast-response power supply (Kepco OPS-3500, Flushing, NY), modulated using a pulse generator constructed in-house. Pulse width and frequency were maintained at 5.0 $\mathrm{ms}$ and $50 \mathrm{~Hz}$ respectively, giving a duty cycle of $25 \%$. The applied voltage was measured using a high voltage probe (Tektronix P6015, Beaverton, NY) with $1 \mathrm{M} \Omega$, $20 \mathrm{pF}$ termination to an oscilloscope (Tektronix 2232, Beaverton, NY). The current was measured using a current meter in line with the power supply. The pressure was determined with a thermocouple vacuum gauge (Teledyne Hastings, Hampton, VA). 

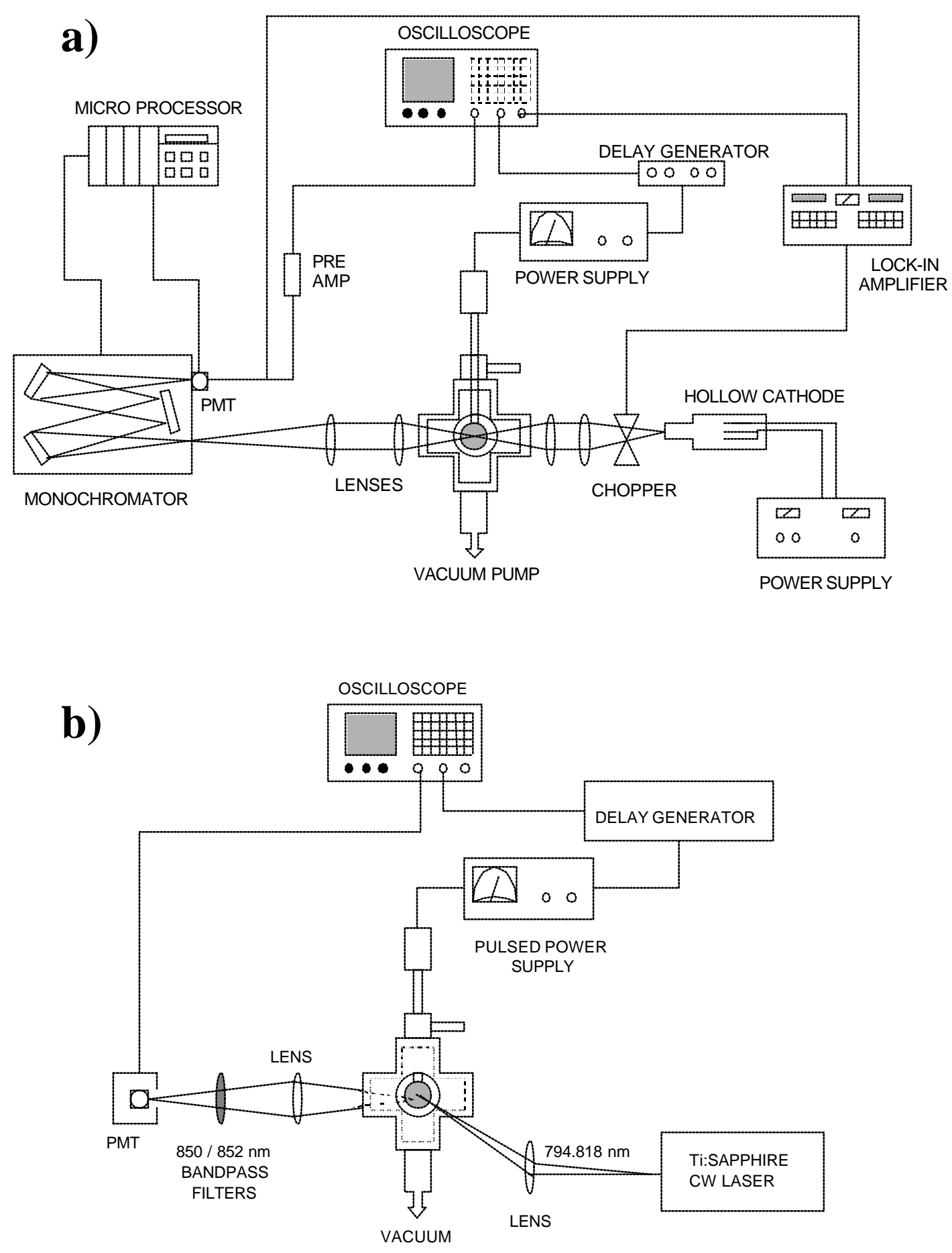

Fig. 2.1. Schematic of a) the orthogonal glow discharge system used for emission and absorbance measurements and b) the system used for laser induced fluorescence.

Reprinted from G.P. Jackson et al., Spectrochim. Acta, Part B, 56(12) (2001) 2449-2464 


\subsubsection{Emission experiments}

For optical emission experiments two plano-convex lenses imaged the light in a 1:1 ratio onto the entrance slit of a $0.64 \mathrm{~m}$ monochromator (ISA HR-640, Edison, NJ). Entrance and exit slits were $50 \mu \mathrm{m}$ wide and $1 \mathrm{~mm}$ high throughout the study. The radiation was dispersed on a 1200 groove $\mathrm{mm}^{-1}$ holographic grating and detected using an extended-red photomultiplier tube (Hamamatsu R-928, Bridgewater, NJ). The output was monitored by an oscilloscope (Lecroy 9370M, Chestnut Ridge, NY) with $1 \mathrm{M} \Omega$ termination. Software (Lecroy Scope Explorer) was used to transform the data into ASCII format.

\subsubsection{Absorbance measurements}

For absorbance measurements, a mechanical chopper (EG\&G197, Princeton, NJ) modulated the incident beam from the hollow cathode lamp (Beckman $\mathrm{Cu}-\mathrm{Ne} \mathrm{HCL}$ for $\mathrm{Cu}$ lines and B-Ar for Ar lines). The hollow cathode lamps (HCL's) operated at $15 \mathrm{~mA}$ constant current using a dc power supply (Kepco BHK-2000, Flushing, NY). The incident beams were focussed midway through the plasma before collection using the same system described for emission experiments. The signal from the PMT was measured with a lock-in amplifier (LIA) (EG\&G PAR 5210, Princeton, NJ) tuned to the chopper frequency of $3 \mathrm{kHz}$. The output from the LIA was monitored on the oscilloscope (with $50 \Omega$ termination). The smallest time-constant of $1 \mathrm{~ms}$ was utilized to obtain the fastest response time. Because the RC time constant caused distortion of the absorption temporal profiles, a program was written in Microsoft Excel Visual Basic to deconvolute 
the data. The deconvolution process is described in the appendix. At each position within the plasma 2500 data points were collected over a $20 \mathrm{~ms}$ time period, and were smoothed twice with a moving average of 10 data points.

The six-way cross housing the GD was mounted on an adjustable support such that the housing could be moved orthogonally with respect to the monochromator axis. To vary the height above the cathode, the insertion probe was carefully maneuvered up and down. In this way, the optics remained constant throughout data acquisition to reduce the risk of skewed results. Data was collected at 368 spatial positions for each map, up to $18 \mathrm{~mm}$ vertically and $8 \mathrm{~mm}$ horizontally. ${ }^{38}$ Resolution is lower at positions that are far from the cathode where it is assumed that dramatic changes in populations do not occur. A program was written in Microsoft Excel Visual Basic to process the large number of data files used for each map. The maps generated with the program were manually verified to ensure that the data had been transferred correctly.

\subsubsection{Laser induced atomic fluorescence}

For LIF experiments a different experimental setup was utilized (see Figure 1b). The glow discharge chamber was a six-way, high vacuum, stainless steel cross (MDC, Hayward, CA). Three side ports on the cross were fitted with optical view ports (Heraeus Suprasil Quartz, Duluth, GA). The fourth side port was used as both a gas inlet and vacuum. A Pirani pressure gauge was used for pressure monitoring. The top port is used for introduction of the cathode direct insertion probe. The cathode was a $6 \mathrm{~mm}$ diameter, $1 \mathrm{~mm}$ thick copper disk attached to the probe using silver paint, and isolated from the 
probe sides using a concentric Macor cylinder. The chamber mount allowed for vertical and side-to-side translation (along the axis of the laser excitation beam).

The discharge pulse was triggered as follows: A delay generator (Stanford Research Systems DG535, Palo Alto, CA) defined the discharge pulse duration. A $5.0 \mathrm{~ms}$ output pulse from the delay generator triggered the glow discharge high voltage power supply (Kepco OPS-3500, Flushing, NY). The resultant $5.0 \mathrm{~ms}$ glow discharge pulse operated at $50 \mathrm{~Hz}$ and ca. $1 \mathrm{kV}$.

The metastable argon atom $1 \mathrm{~s}_{3}-2 \mathrm{p}_{4}$ transition (see Figure 2) was excited using an Ar ion laser pumped titanium sapphire laser (Spectra Physics 3900) tuned to $794.818 \mathrm{~nm}$. The incident light $(75 \mathrm{~mW})$ was focused into the discharge cell with a $250 \mathrm{~mm}$ focal length planoconvex lens, and was aligned to pass directly beneath the center of the glow discharge cathode. Laser induced fluorescence from the $2 \mathrm{p}_{4}-1 \mathrm{~s}_{2}$ transition at $852.144 \mathrm{~nm}$ was collected at 90 degrees with respect to excitation by a $50 \mathrm{~mm}$ diameter, $250 \mathrm{~mm}$ focal length biconvex lens. The lens was positioned equidistant between the cathode and detector for one-to-one imaging of the discharge. Collected light passed through two narrow bandpass filters ( $850 \mathrm{~nm}$ by $10 \mathrm{~nm}$ bandpass, and $852 \mathrm{~nm}$ by $5 \mathrm{~nm}$ bandpass) used for rejection of the plasma background emission. The collected light was imaged on a 1 mm pinhole (to provide a spatial mask) and is detected by a PMT (R955, Hamamatsu Corp.) located directly behind the pinhole. The PMT signal response was confirmed to be linear over the range of signal intensities observed.

Output from the PMT was acquired with a digital storage oscilloscope (Tektronix TDS 520C, Beaverton, OR) using a $1 \mathrm{M} \Omega$ input impedance and $4 \mu$ s per point timebase resolution. The scope was triggered synchronously with the discharge using the delay 
generator. An average of 256 traces were acquired at each plasma position and transferred to a PC for further analysis. Even with the use of 2 rejection filters in the collection path, significant plasma background was observed in the wavelength region of the fluorescence signal. Emission traces were obtained at each plasma position with and without laser excitation for background subtraction. Data were obtained at the same spatial positions as described for the emission and absorption experiments. Whereas the Absorption and emission data were collected in horizontal slices, LIF data were collected in vertical slices by moving the direct insertion probe before moving the housing to the next horizontal position. At the end of each vertical slice the plasma was returned to the $(0,0)$ position to measure the extent of drift. Each vertical dimension could then be normalized to account for any drift in incident laser wavelength (which resulted in a drop in LIF intensity).

\subsection{Results and Discussion}

This study considers the two metastable argon atom states- $\left(1 \mathrm{~s}_{5}\right){ }^{3} \mathrm{P}_{2}$ at $11.55 \mathrm{eV}$ and $\left(1 \mathrm{~s}_{3}\right){ }^{3} \mathrm{P}_{0}$ at $11.72 \mathrm{eV}$ - separately because of their different formation and quenching rates. $^{32,39-42}$ Figure 2.2 shows a simplified energy level scheme of the transitions used to monitor the two metastable states. Absorbance and emission measurements were made of both the $794.8 \mathrm{~nm}$ and $811.5 \mathrm{~nm}$ lines along with laser pumping at $794.8 \mathrm{~nm}$ followed by emission at $852.1 \mathrm{~nm}$. 


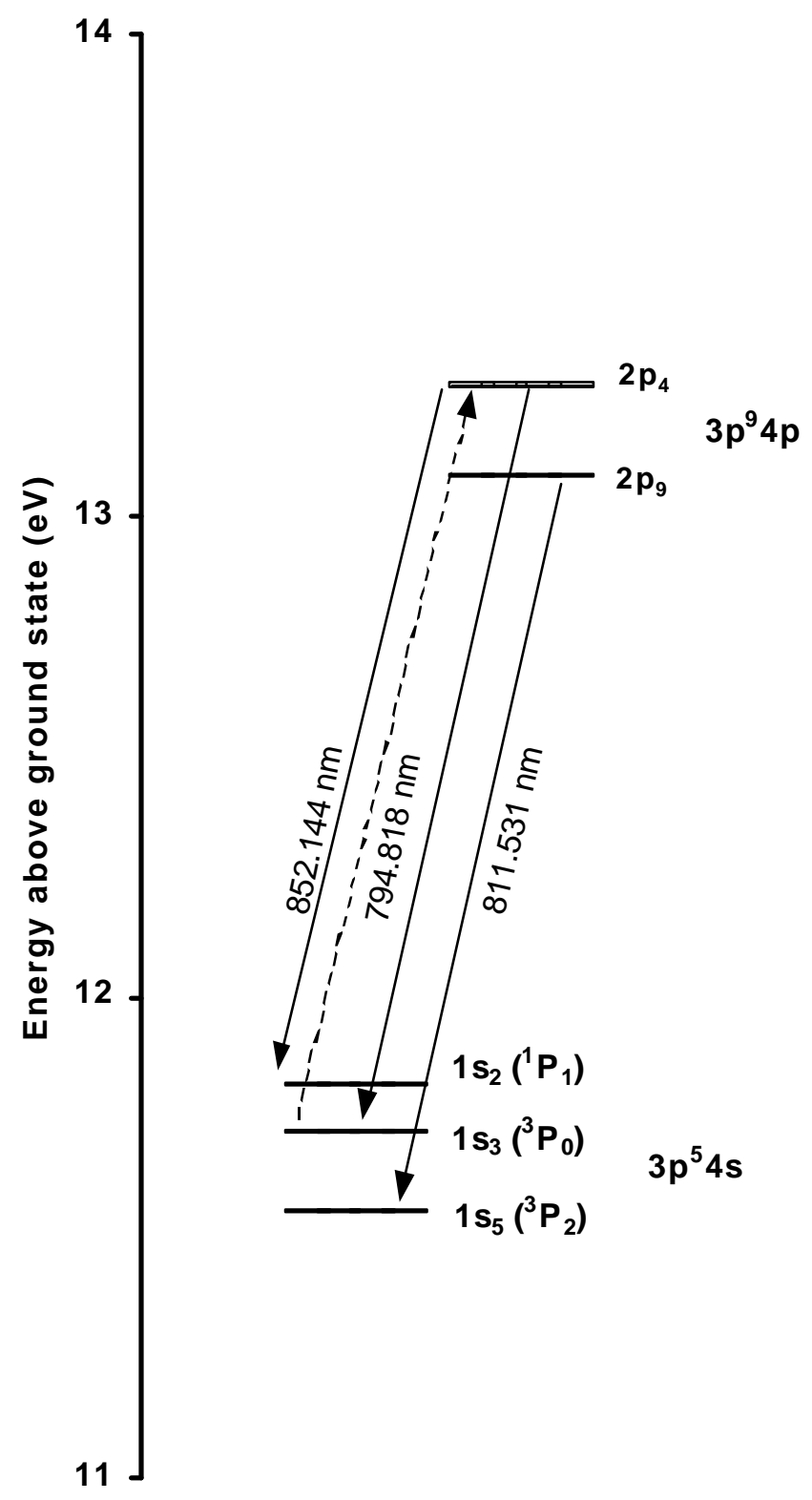

Fig. 2.2. Energy level scheme and transitions, relevant to this experiment, for the argon atom metastable states of ${ }^{3} \mathrm{P}_{2}(11.55 \mathrm{eV})$ and ${ }^{3} \mathrm{P}_{0}(11.72 \mathrm{eV})$. Reprinted from G.P. Jackson et al., Spectrochim. Acta, Part B, 56(12) (2001) 2449-2464 
Figure 2.3.a shows a typical applied voltage pulse as used for the absorption and emission experiments (the applied voltage for the LIF experiments was essentially a square wave, having a fall time $<8 \mu$ s, and is not presented). Because the rise time is approximately 1 $\mathrm{ms}$, a $5.0 \mathrm{~ms}$ pulse width ensures that steady state conditions are reached. The fast fall time aids in the formation of an afterpeak ${ }^{29}$ and helps eliminate processes, such as electron excitation, that could be stimulated by the presence of an electric field.

Figure 2.3.b shows a typical temporal absorption profile taken $5.0 \mathrm{~mm}$ above the center of the cathode, before deconvolution, for the $811.5 \mathrm{~nm}$ transition. The effects of the $1 \mathrm{~ms}$ time constant in the LIA are evident in that the profile never reaches a plateau and the afterpeak is delayed and broadened. Deconvolution of the data (see appendix) yields the trace shown in Figure 2.3.c. In this case, steady state conditions appear shortly after $2 \mathrm{~ms}$, the afterpeak is more apparent, and the afterpeak shifts to a shorter time of 5.7 ms. The absorption plot shown clearly indicates an increase in the metastable argon atom population during the afterpeak time regime.

The emission at $811.5 \mathrm{~nm}$ (Figure 2.3.d), also taken at $5 \mathrm{~mm}$, corresponds to the argon atom transition from $2 \mathrm{p}_{4}-1 \mathrm{~s}_{5}, 13.08-11.55 \mathrm{eV}$, and represents the highest transition probability for formation of the ${ }^{3} \mathrm{P}_{2}$ metastable. Shortly after pulse termination, a noticeable afterpeak appears at $5.35 \mathrm{~ms}$, just before the maximum in the absorbance value. This observation is consistent with the formation of metastable argon atoms via radiative decay. 


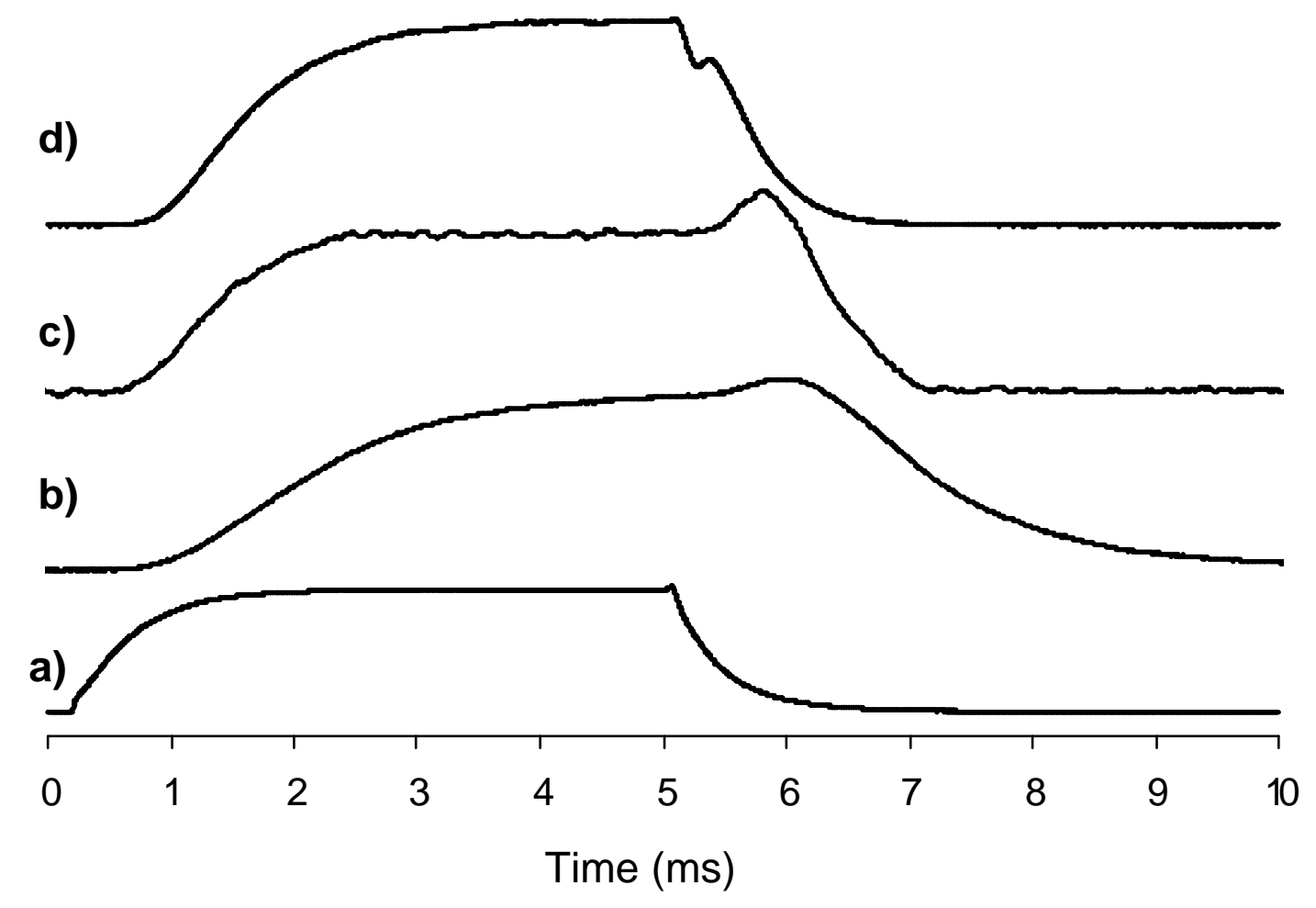

Fig. 2.3. Temporal profiles showing a) The applied voltage b) raw absorbance data at $811.5 \mathrm{~nm}$ before deconvolution and c) the same data after deconvolution and smoothing twice with a moving average of 10 points and d) emission profile for $811.5 \mathrm{~nm}$. Conditions; $5 \mathrm{~ms}$ pulse, $25 \%$ duty cycle $(50 \mathrm{~Hz}), 0.8$ Torr, $1.8 \mathrm{~mA}$ peak current, $860 \mathrm{~V}$ peak voltage ( 1.5 W peak power), $5 \mathrm{~mm}$ above cathode center. Reprinted from G.P. Jackson et al., Spectrochim. Acta, Part B, 56(12) (2001) 2449-2464 


\subsection{1. ${ }^{3} \mathrm{P}_{2}$ Argon atom metastables: population via radiative decay}

Figure 2.4 shows how the emission intensity for the $811.5 \mathrm{~nm}$ line varies as a function of time from $1.5 \mathrm{~ms}$ to $5.7 \mathrm{~ms}$. This emission corresponds to the $13.08-11.55 \mathrm{eV}, 2 \mathrm{p}_{9}-1 \mathrm{~s}_{5}$ $\left({ }^{3} \mathrm{D}_{3}{ }^{3} \mathrm{P}_{2}\right)$ transition and exhibits the strongest emission for a transition resulting in the population of the ${ }^{3} \mathrm{P}_{2}$ metastable level. At $1.5 \mathrm{~ms}$, the plasma is still forming and the emission intensity is still increasing. From $2.0-5.0 \mathrm{~ms}$, steady state conditions are established and the emission characteristics do not change. During this steady state condition, emission intensity is low immediately at the cathode and increases rapidly to a maximum 1.0-1.5 mm above the surface. The intensity decreases rapidly from 1.5-2.5 $\mathrm{mm}$ but decreases more slowly at distances greater than $2.5 \mathrm{~mm}$. This behavior is similar to observations made by Hoppstock and Harrison for a glow discharge operating under steady state conditions ${ }^{43}$. The intense emission focused around $1 \mathrm{~mm}$ defines the negative glow directly adjacent to the cathode dark space. In steady state discharges, the $4 \mathrm{p}$ states are in a high state of flux and are thought to be populated in almost equivalent amounts by electron excitation from the ground state, electron excitation from a $4 \mathrm{~s}$ state and by

radiative decay from higher levels. ${ }^{44}$ Significant depopulation processes include radiative decay to $4 \mathrm{~s}$ levels (as observed by the $811.5 \mathrm{~nm}$ emission in this case) and electron excitation to higher levels. Notice that the $4 \mathrm{~s}$ levels and highly excited states thus formed are capable of repopulating the $4 p$ levels. 


\section{$\underset{\text { Key }}{\square} 90-100 \% \square 70-80 \% \square 50-60 \% \square 30-40 \% \square 10-20 \%$ 口 $80-90 \% \square 60-70 \% \square 40-50 \% \square 20-30 \% \square 0-10 \%$}
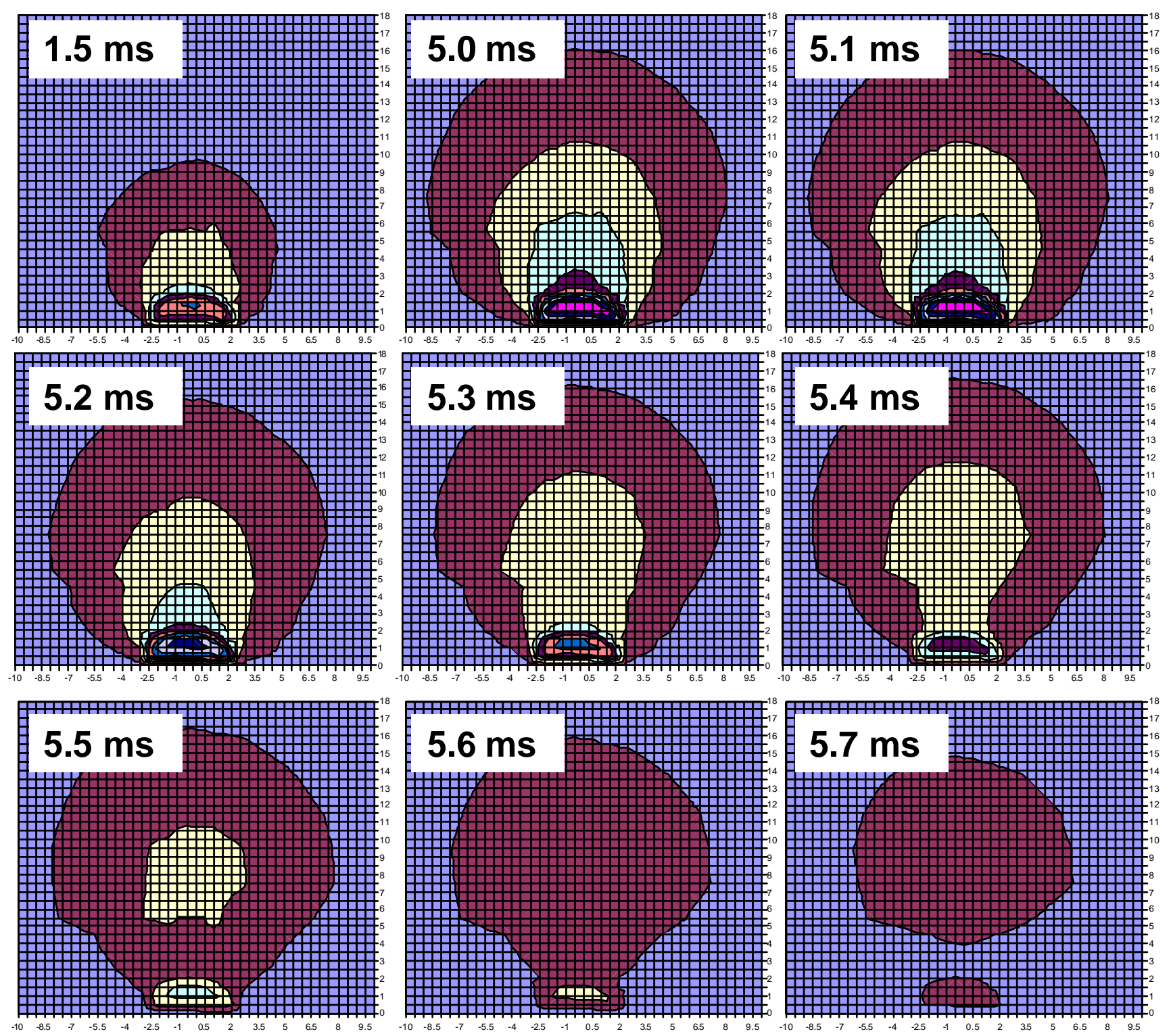

Fig. 2.4. Two-dimensional images of argon atom emission at $811.5 \mathrm{~nm}$. Conditions; $5 \mathrm{~ms}$

pulse, $25 \%$ duty cycle $(50 \mathrm{~Hz}), 0.8$ Torr, $1.8 \mathrm{~mA}$ peak current, $860 \mathrm{~V}$ peak voltage $(\sim 1.5$

W peak power). Reprinted from G.P. Jackson et al., Spectrochim. Acta, Part B, 56(12)

(2001) 2449-2464 
Upon power termination the emission intensity within $4 \mathrm{~mm}$ of the cathode simply decreases and shows no post-pulse enhancement. The region positioned $6 \mathrm{~mm}$ above the cathode remains almost constant between 5.0-5.5 ms. The area between 7-8 $\mathrm{mm}$ can be seen to decrease in intensity between $5.0-5.3 \mathrm{~ms}$, but then increase through 5.4-5.5 ms. Because the emission intensity actually increases, the $4 \mathrm{p}$ population processes must be enhanced in this vicinity from 5.3-5.5 ms. This leads us to propose a method of populating the $\sim 13 \mathrm{eV}$ levels in the absence of a strong electric field. Because there are no strong coulombic forces with which to accelerate unbound electrons, electron excitation can be ruled out (for the same reason, depopulation of the $2 \mathrm{p}_{9}$ state via electron excitation to higher levels is also reduced). The significant mechanisms remaining with which to feed this state are radiative decay and collisional deexcitation from higher excited states. The highly excited states are also unable to be populated by electron excitation, however, so must be populated via electron ion recombination. The main process leading to the observed increase of emissions at $811.5 \mathrm{~nm}$ in the afterpeak is expected to commence with both radiative and collisional recombinations of argon ions (formed during power application) and thermal electrons. 


\subsection{2. ${ }^{3} \mathrm{P}_{2}$ Argon atom metastables: Atomic absorption measurements}

The absorbance at $811.5 \mathrm{~nm}$ accompanies the transition from the $\left({ }^{3} \mathrm{P}_{2}\right) 1 \mathrm{~s}_{5}$ metastable state to the $2 \mathrm{p}_{9}$ state. The population fluctuations in time are shown in twodimensional maps in Figure 2.5. During steady state conditions this metastable argon atom state is most abundant $1 \mathrm{~mm}$ above the cathode- in agreement with the emission data. After pulse termination the distribution of metastable argon atoms changes dramatically, and is similar to that observed in the emission profile, although the absorbance times are delayed. From 5.0-5.6 ms the metastable population hardly changes throughout the plasma. After this time, the bulk population can be seen to move away from the cathode. This movement coincides with the formation trends observed in the $811.5 \mathrm{~nm}$ emission maps. Close inspection of the $5.9 \mathrm{~ms}$ map in the region 4-8 $\mathrm{mm}$ away from the cathode reveals how the density of metastable argon atoms actually increases by approximately $20 \%$ compared to the steady state conditions. Klingler et l. $^{29}$ did not observe an increase in the afterpeak absorbance for the same transition under similar conditions. However, they did not give a specific distance at which they monitored the absorbance of this metastable. It is possible that their measurements were made in the beginning of the negative glow (2-3 $\mathrm{mm}$ from the cathode surface) where there is no increase in number density in the afterpeak compared to the plateau. The maximum abundance after $5.9 \mathrm{~ms}$ is found between $5-8 \mathrm{~mm}$. At $9 \mathrm{~mm}$ above the cathode surface, the metastable density $1.4 \mathrm{~ms}$ after pulse termination is within $10 \%$ of the density during the plateau. In contrast, the density $1 \mathrm{~mm}$ above the cathode decreases by almost $70 \%$ over the same time period. 
It should be remembered that the temporal accuracy of the absorbance data is somewhat suspect because of the RC time constant effect discussed above. Deconvolution helped remove some of the time lag but it is considered that the time domain can be used more as a relative index rather than absolute for these measurements. Nonetheless, the overall trends, however, clearly indicate that the metastable argon atom distribution in the afterglow is very different from that of steady state conditions and that the population maximum is found $47 \mathrm{~mm}$ further away from the cathode surface than during steady state conditions. It is not surprising that processes dependent on this metastable state, such as Penning ionization of analyte species, would also reflect this shift. $^{45}$ 
Key

$\square 90-100 \% \square 70-80 \% \square 50-60 \% \square 30-40 \% \square 10-20 \%$ 口 $80-90 \% \square 60-70 \% \square 40-50 \% \square 20-30 \% \square 0-10 \%$
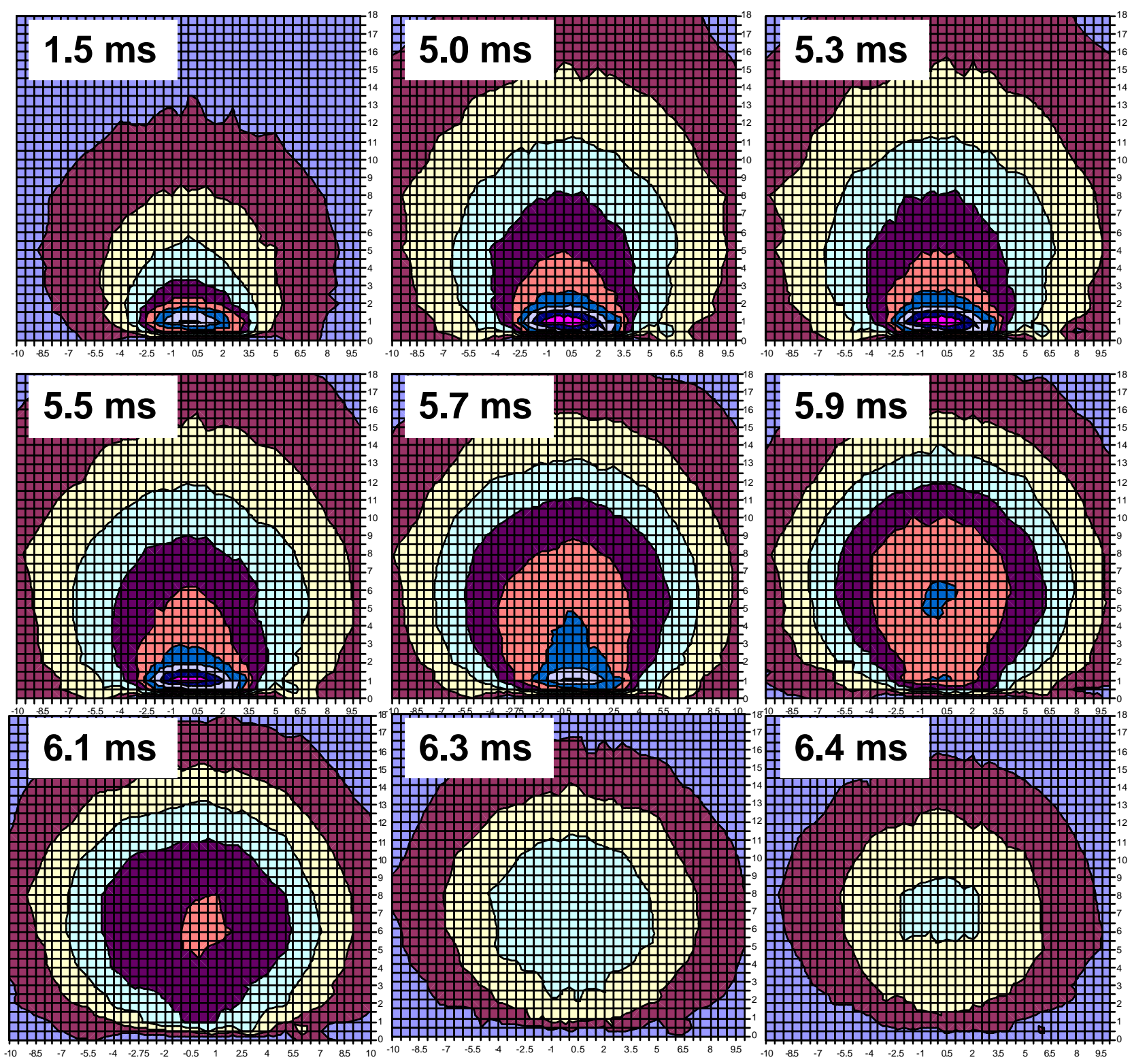

Fig. 2.5. Two-dimensional images of argon atom absorption at $811.5 \mathrm{~nm}$. Conditions; 5 ms pulse, $25 \%$ duty cycle $(50 \mathrm{~Hz}), 0.8$ Torr, $1.8 \mathrm{~mA}$ peak current, $860 \mathrm{~V}$ peak voltage ( $1.5 \mathrm{~W}$ peak power). Reprinted from G.P. Jackson et al., Spectrochim. Acta, Part B, 56(12) (2001) 2449-2464 


\subsection{3. ${ }^{3} P_{0}$ Argon atom metastables: Population via radiative decay}

The emission at $794.8 \mathrm{~nm}$, Figure 2.6, corresponds with the transition from $2 \mathrm{p}_{4}$ $1 \mathrm{~s}_{3}, 13.28-11.72 \mathrm{eV}$. Again, intensities are normalized to the largest intensity over the entire pulse cycle. During steady state conditions (i.e. from 3-5ms) the spatial emission distribution is almost identical to that of the $811.5 \mathrm{~nm}$ emission, although the absolute emission intensity is obviously lower (the ${ }^{3} \mathrm{P}_{0}$ level has a degeneracy of 1 , while the ${ }^{3} \mathrm{P}_{2}$ level has a degeneracy of 5 , and the transition probability is lower for the former: $0.186 \mathrm{x}$ $10^{8}$ vs. $\left.0.331 \times 10^{8} \mathrm{~s}^{-1}\right)^{46}$. After pulse termination at $5.0 \mathrm{~ms}$, the emission intensity decays in a fashion similar to the $811.5 \mathrm{~nm}$ line: within $4 \mathrm{~mm}$ the emission intensity steadily declines, between 6-8 $\mathrm{mm}$ the intensity initially decreases before increasing, and the plasma beyond $9 \mathrm{~mm}$ remains relatively constant. Not accounting for metastable loss processes, one would expect the emission maps of Figure 2.6 to precede the ${ }^{3} \mathrm{P}_{0}$ population in the afterpeak. 


\section{Key \\ $\square 90-100 \% \square 70-80 \% \square 50-60 \% \square 30-40 \% \square 10-20 \%$ 口 $80-90 \% \square 60-70 \% \square 40-50 \% \square 20-30 \% \square 0-10 \%$}

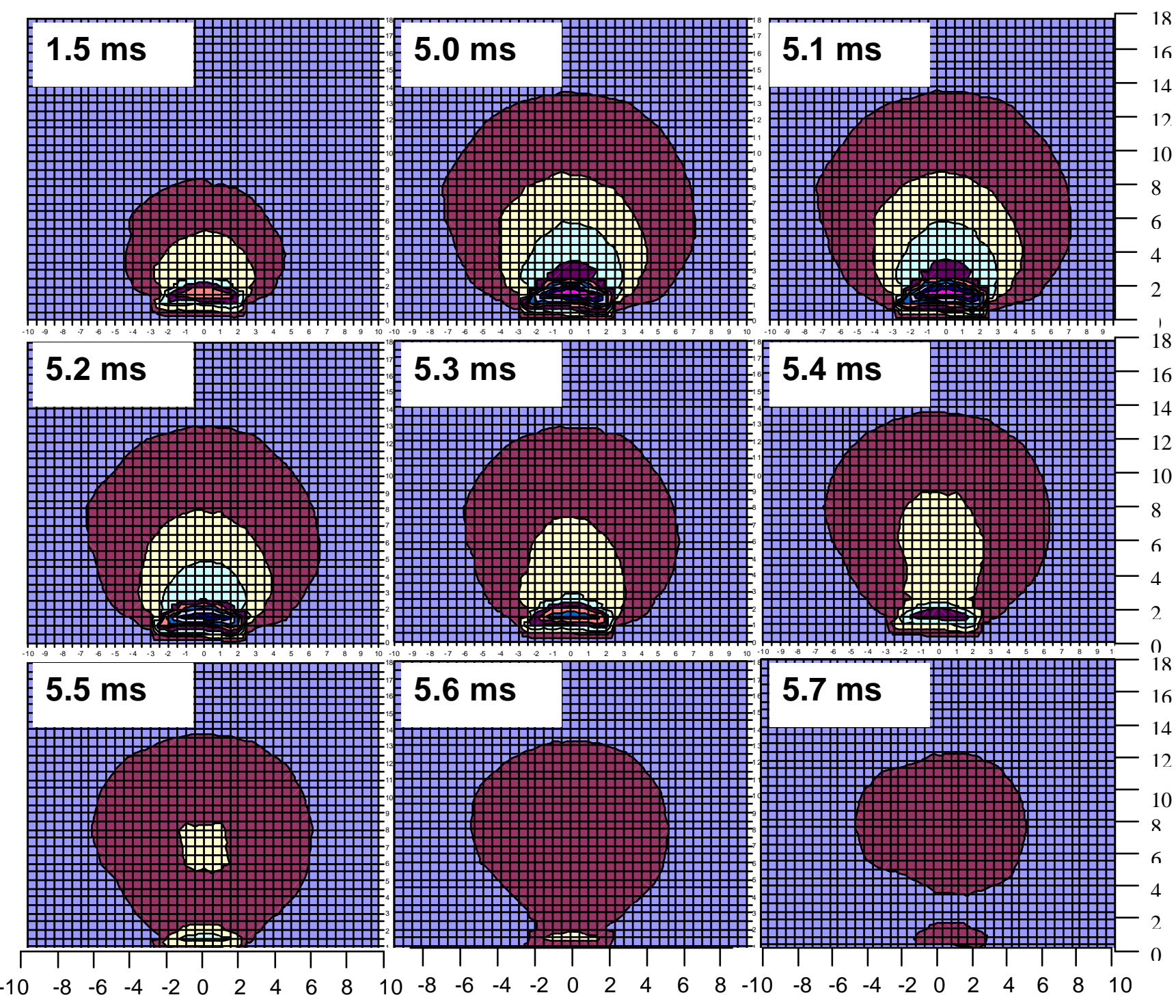

Fig. 2.6. Two-dimensional images of argon atom emission at $794.8 \mathrm{~nm}$. Conditions; $5 \mathrm{~ms}$ pulse, $25 \%$ duty cycle $(50 \mathrm{~Hz}), 0.8$ Torr, $1.8 \mathrm{~mA}$ peak current, $860 \mathrm{~V}$ peak voltage $(\sim 1.5$ W peak power). Reprinted from G.P. Jackson et al., Spectrochim. Acta, Part B, 56(12) (2001) 2449-2464 


\subsection{4. ${ }^{3} P_{0}$ Argon atom metastables: Absorption and laser induced fluorescence}

\section{measurements}

A spatial map for the absorbance at $794.8 \mathrm{~nm}$ and $4.0 \mathrm{~ms}$ appears in Figure 2.7.a. The absorption was found to be steady-state at this time. A spatial map for the LIF of this metastable state at $4.0 \mathrm{~ms}$ appears in Figure 2.7.b. In both maps the data have been normalized to the largest value in their respective pulse cycles. The spatial similarities between the two maps are indicative of the reproducibility of this experiment on two entirely different systems. In both cases the maximum population is found about $1.0 \mathrm{~mm}$ above the cathode, in agreement with the emission map (at $794.8 \mathrm{~nm}$ ) and previous work by Bogaerts et al. on this species under steady-state conditions. ${ }^{6}$ The slightly less symmetrical appearance of the LIF map may arise from incomplete intensity correction to compensate for the drift in wavelength of the excitation laser (see experimental).

In contrast to Bogaerts et al.- who observed a second population of this metastable state further from the cathode- a second population under steady state conditions is not visible in these experiments. This could be because the cell geometry and operating conditions used in the present study differ from those examined by Bogaerts et al. It is interesting to note that in this present study the maximum emission, absorbance and LIF values for both metastable levels appear at a distance where Ferreira et al. find the metastable populations to reach a minimum. ${ }^{24}$ Ferreira et al. find absorbance maxima at the cathode surface and ca $3 \mathrm{~mm}$ above the surface. The operating

pressure had a dramatic effect on the spatial position and density of the ${ }^{3} \mathrm{P}_{2}$ metastable atom. 

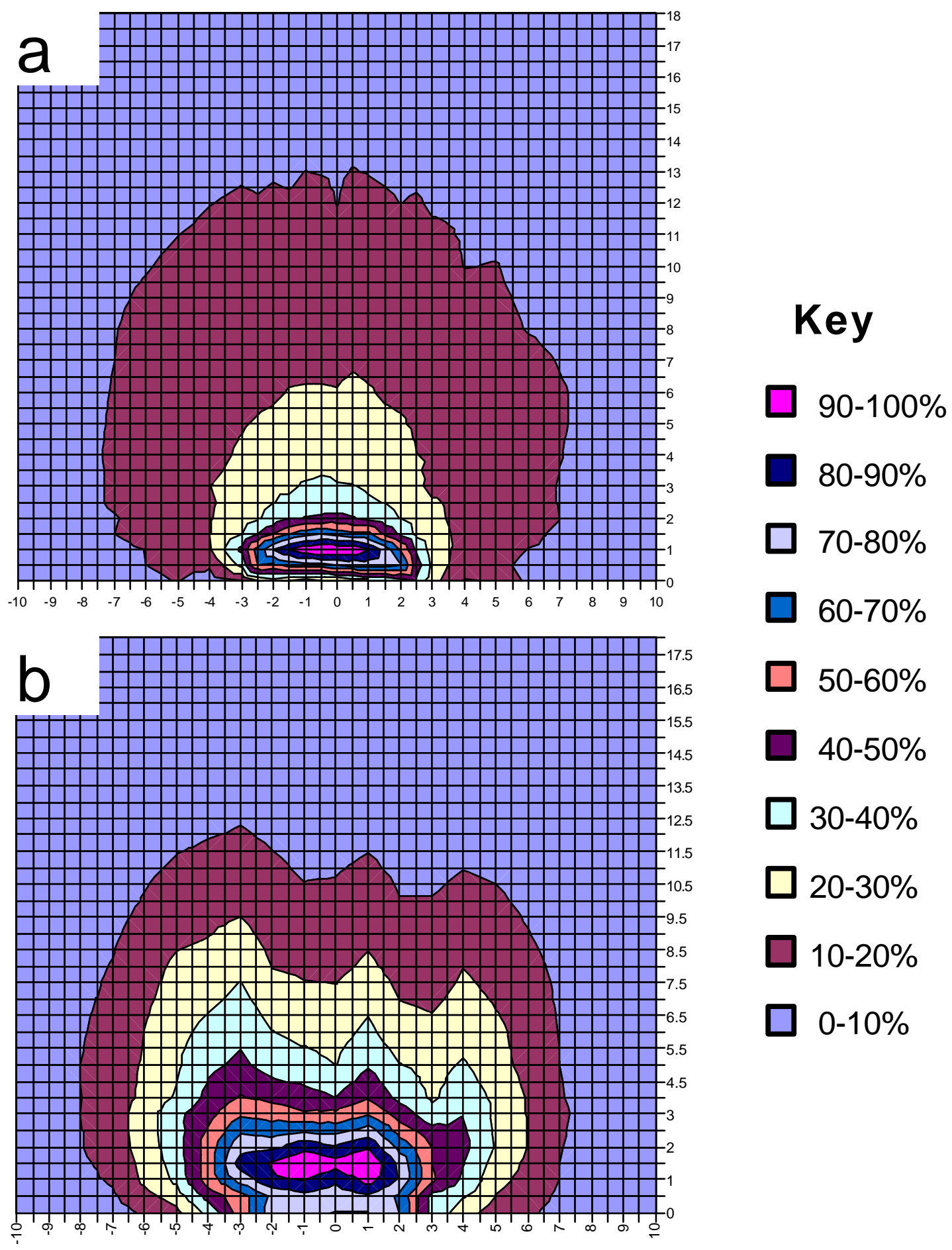

Fig. 2.7. Two-dimensional images of the ${ }^{3} \mathrm{P}_{0}$ metastable argon atom state at $4.0 \mathrm{~ms}$ by a) absorption at $794.8 \mathrm{~nm}$ and b) Laser induced fluorescence (see experimental). Reprinted from G.P. Jackson et al., Spectrochim. Acta, Part B, 56(12) (2001) 2449-2464. 
Bogaerts et $a l .{ }^{6}$ noted that populations did not shift in space as a function of pressure and that the maxima were observed at ca. $4.0 \mathrm{~mm}$ and $12 \mathrm{~mm}$. Again, such contrasting results reinforce the fact that no single descriptive model accurately addresses the different operating conditions and cell geometries of GD sources.

In the afterpeak the population of the ${ }^{3} \mathrm{P}_{0}$ metastable state- as measured by absorbance and LIF- displays a smaller post-pulse increase than is seen for the absorption at $811.5 \mathrm{~nm}$ for the ${ }^{3} \mathrm{P}_{2}$ state (reasoning is provided at the end of this section). When viewed in the two-dimensional-map form, therefore, LIF and absorption data do not show such a significant shift in the ${ }^{3} \mathrm{P}_{0}$ state between the plateau and afterpeak time regimes. If, however, the ratio of afterpeak/plateau values are plotted as a function of distance from the cathode (Figure 2.8) it is evident that the ${ }^{3} \mathrm{P}_{0}$ state is enhanced in certain areas of the afterpeak plasma. The absorbance afterpeak/plateau ratio at $7 \mathrm{~mm}$ is greater than 1 and implies that the number density is greater in the afterpeak than the plateau. The LIF afterpeak/plateau ratios maximize in approximately the same region $(6 \mathrm{~mm}$ above the cathode) and again identify the post pulse increase in population of this species. The slight difference in the afterpeak/plateau maxima positions between the LIF and absorbance data is probably due to the difference in fall time of the applied pulse. As mentioned in the experimental, the fall times in the LIF and absorption experiments are $\sim 8 \mu \mathrm{s}$ and $\sim 800 \mu \mathrm{s}$ respectively. The difference in voltage fall times is also attributed to the faster appearance of an afterpeak in the LIF experiments. The afterpeak was found to maximize $\sim 200 \mu$ s after pulse termination in the LIF study but $\sim 400 \mu$ s in the emission study. 


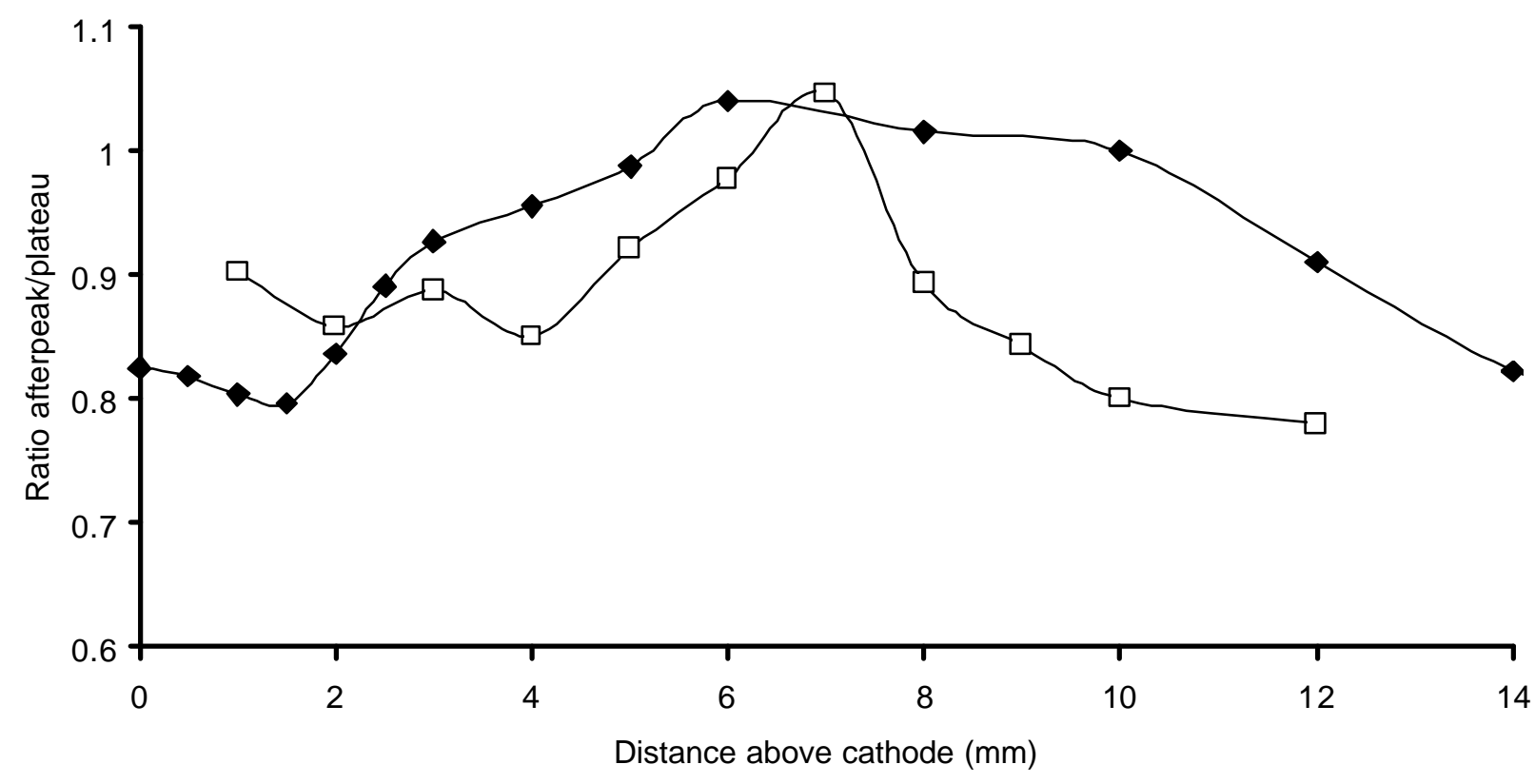

Fig. 2.8. Ratio of afterpeak/plateau values for the ${ }^{3} \mathrm{P}_{0}$ metastable argon atom state by absorption at $794.8 \mathrm{~nm}[5.5 \mathrm{~ms} / 4.0 \mathrm{~ms}]$ and $\bullet$ Laser induced fluorescence $[\sim 5.15$ ms/4.0ms]. Reprinted from G.P. Jackson et al., Spectrochim. Acta, Part B, 56(12) (2001) 2449-2464 
Both absorbance and LIF measurements show smaller afterpeak/plateau ratio values close to the cathode. This reflects the lack of ${ }^{3} \mathrm{P}_{0}$ state formation and/or greater loss mechanisms in this spatial region for the afterpeak. This same phenomenon was observed for the ${ }^{3} \mathrm{P}_{2}$ state. An enhanced loss mechanism specific to this proximity is likely to be diffusion to and deexcitation at the cathode surface.

The afterpeak increase of the ${ }^{3} \mathrm{P}_{0}$ state in absorption measurements is less easily defined than that of the ${ }^{3} \mathrm{P}_{2}$ state, in these experiments. Unless the weaker afterpeak for the ${ }^{3} \mathrm{P}_{0}$ state is due to some unforeseen experimental error, the ratio of the ${ }^{3} \mathrm{P}_{2} /{ }^{3} \mathrm{P}_{0}$ metastable levels differs quite considerably between the plateau and afterpeak time regimes. (A reviewer has pointed out that a change in the relative magnitudes of the metastable states is consistent with the reported literature on switching from an ionizing plasma to a recombining plasma. This phenomenon is discussed in detail in Chapter 3). It is safe to assume that electron-ion recombination (two or three body) followed by radiative decay is the dominant gain mechanism for both levels in the afterpeak. The ${ }^{3} \mathrm{P}_{2}$ state could therefore be more highly populated in the afterpeak if the sum of the radiative transition probabilities to the ${ }^{3} \mathrm{P}_{2}$ state exceed those to the ${ }^{3} \mathrm{P}_{0}$ state. An approximation for this calculation uses the transition probabilities and degeneracies of the 15 strongest lines leading to the formation of each metastable. The result indicates that the ${ }^{3} \mathrm{P}_{2}$ state is more probably populated than ${ }^{3} \mathrm{P}_{0}$ state by approximately one order of magnitude. The population processes for the two metastable species in the steady-state and afterpeak times are therefore different enough that the ${ }^{3} \mathrm{P}_{2}$ contributes a larger proportion of the total metastable argon atom population in the afterpeak. This finding is significant if 
mathematical models such as those proposed by Bogearts et al. $^{6}$ are based on measurements on the less-abundant ${ }^{3} \mathrm{P}_{0}$ state.

\subsection{Conclusions}

During steady state, plateau, conditions the populations of both ${ }^{3} \mathrm{P}_{2}$ and ${ }^{3} \mathrm{P}_{0}$ argon atom states maximize 1-2 $\mathrm{mm}$ above the cathode surface. This is in general agreement with other investigations of these species in analytical glow discharges operating under similar conditions. Population processes are likely to be electron impact and fast atom/ion impact at this distance. In the afterglow, the bulk of both the ${ }^{3} \mathrm{P}_{2}$ and ${ }^{3} \mathrm{P}_{0}$ metastable states are formed 4-7 mm further from the cathode surface than during the plateau. Argon ionelectron recombination followed by radiative relaxation is the most probable mechanism of populating these levels. Ongoing work in our laboratory indicates that excitation and ionization of sputtered species in the afterglow is extremely dependent on these metastable argon atoms. Finally, this study has shown that the observation time and position both dramatically affect the behavior observed for species in pulsed GD plasmas. 


\subsection{Appendix}

\subsubsection{Theory}

The deconvolution process is based on a procedure described by Jansson. ${ }^{47}$ The smallest RC time constant of $1 \mathrm{~ms}$ was selected when collecting absorbance data with the lock-in amplifier. This time constant is on the order of the pulse profiles used in this study and has the effect of distorting the input signal voltage according to the equation

$$
v_{c}(t)=\xi(t) \otimes v(t)
$$

(assuming a first order [linear] approximation of the RC time constant) where $v_{c}(t)$ is the output signal voltage, $v(\mathrm{t})$ is the input voltage and $\xi(\mathrm{t})$ is given by

$$
\xi(t)=(1 / R C) H(t) \exp (-t / R C)
$$

where $\mathrm{RC}$ is the time constant $\left(1 \times 10^{-3} \mathrm{~s}\right), \mathrm{H}(\mathrm{t})$ is the heavy side step function, and $\mathrm{t}$ is the time. This can also be written in the form

$$
\mathrm{V}_{\mathrm{c}}(\omega)=\Xi(\omega) \mathrm{V}(\omega)
$$

where $V_{c}(\omega)$ and $V(\omega)$ are the frequency spectra of the output and input voltages, respectively, and the complex filter transfer function is given by

$$
\Xi(\omega)=1 /(2 \pi)^{1 / 2}(1-\mathrm{j} \omega \mathrm{RC}) /(1+\omega \mathrm{RC})^{2}
$$

\subsubsection{Application of theory}

The time axis is first modified with the heavy side step function $\left(t_{i}=\exp -t_{i} / R C\right)$. Next, FFT's (fast Fourier transforms) of $2^{\text {n }}$ (i.e. 2048, or 4096) data points were performed on both the modified time axis $[\mathrm{H}(\mathrm{t})]$ and the digitized voltage output $[\mathrm{G}(\mathrm{t})]$. An inverse FFT was performed on the quotient $\Xi[\mathrm{H}(\mathrm{t})] / \Xi[\mathrm{G}(\mathrm{t})]$ to obtain the 
deconvoluted data $[v(t)]$. After baseline correction the deconvoluted data was averaged twice with a moving average of ten data points before being exported to a database.

As with any Fourier analysis, it was found that using a larger number of data points enhanced the deconvolution process. Unfortunately, with our current FFT software (Microsoft Excel), we are restricted to 4096 data points. A program was written in Microsoft Visual Basic in order to deal with the huge number of data files. Each ASCII file was imported individually into a template. After the above mathematical analysis, the deconvoluted data were transferred to a database file before importing the next raw data file. Because of the nature of the database, intensity values can be extracted to generate a 2D map for any given moment in time (to within $4 \mu \mathrm{s}$ ). 


\subsection{References}

(1) Marcus, R. K. Glow Discharge Spectroscopies, 1 ed.; Plenum Press, 1993.

(2) Bogaerts, A.; Gijbels, R. Spectrochim. Acta, Part B 1998, 53, 1-42.

(3) King, F. L.; Harrison, W. W. Mass Spec. Rev. 1990, 9, 285-213.

(4) Bogaerts, A.; Gijbels, R.; Carman, R. J. Spectrochim. Acta, Part B 1998, 53, 1679-1703.

(5) Bogaerts, A.; Gijbels, R.; Vlcek, J. Spectrochim. Acta, Part B 1998, 53, 15171526.

(6) Bogaerts, A.; Guenard, R. D.; Smith, B. W.; Winefordner, J. D.; Harrison, W. W.; Gijbels, R. Spectrochim. Acta, Part B 1997, 52, 219-229.

(7) Bogaerts, A.; Gijbels, R. J. Am. Soc. Mass Spectrom. 1997, 8, 1021-1029.

(8) Bengtson, A. J. Anal. At. Spectrom. 1996, 11, 829-833.

(9) Chakrabarti, C. L.; Headrick, K. L.; Hutton, J. C.; Bicheng, Z.; Bertels, P. C.; Back, M. H. Anal. Chem. 1990, 62, 574-586.

(10) Bernhard, A. E. Spectroscopy 1987, 2, 24.

(11) Hang, W.; Baker, C.; Smith, B. W.; Winefordner, J. D.; Harrison, W. W. J. Anal. At. Spectrom. 1997, 12, 143-149.

(12) Yang, C.; Ingeneri, K.; Harrison, W. W. J. Anal. At. Spectrom. 1999, 14, 693-698.

(13) Cordos, E.; Malmstadt, H. V. Anal. Chem. 1973, 45, 27-32.

(14) Duncen, H.; Tiller, H. J.; Meisel, J. Spectrochim. Acta, Part B 1969, 24, 632-634.

(15) Butler, L. R. P.; Kroger, K.; West, C. D. Spectrochim. Acta, Part B 1975, 30, 489499. 
(16) Pan, C.; King, F. L. Appl. Spectrosc. 1993, 47, 300-304.

(17) Glick, M.; Smith, B. W.; Winefordner, J. D. Anal. Chem. 1990, 62, 157-161.

(18) Smith, B. W.; Omenetto, N.; Winefordner, J. D. Spectrochim. Acta, Part B 1984, 39, 1389-1393.

(19) Van Dijk, C.; Smith, B. W.; Winefordner, J. D. Spectrochim. Acta, Part B 1982, $37,759-768$.

(20) Strauss, J. A.; Ferreira, N. P.; Human, H. G. C. Spectrochim. Acta, Part B 1982, $37,947-954$.

(21) Hang, W.; Walden, W. O.; Harrison, W. W. Anal. Chem. 1996, 68, 1148-1152.

(22) King, F. L.; Pan, C. Anal. Chem. 1993, 65, 735-739.

(23) Walsh, A. Appl. Spec. 1973, 27, 335-341.

(24) Ferreira, N. P.; Strauss, J. A.; Human, H. G. C. Spectrochim. Acta, Part B 1982, $37,273-279$.

(25) Fujimoto, T. J. Phys. Soc. Japan 1979, 47, 265-272.

(26) Fujimoto, T. J. Phys. Soc. Japan 1979, 47, 273-281.

(27) Pan, C.; King, F. L. Anal. Chem. 1993, 65, 3187-3193.

(28) Pan, C.; King, F. L J. Am. Soc. Mass Spectrom. 1993, 4, 727-732.

(29) Klinger, J. A.; Barshick, C. M.; Harrison, W. W. Anal. Chem. 1991, 63, 25712576.

(30) Lewis, C. L.; Oxley, E. S.; Pan, C. K.; Steiner, R. E.; King, F. L. Anal. Chem. 1999, 71, 230-234.

(31) Bourene, M.; LeCalve, J. J. Chem. Phys 1973, 58, 1452-1458.

(32) Piper, L. G.; Velazco, J. E.; Setser, D. W. J. Chem. Phys. 1973, 59, 3323-3340. 
(33) Steiner, R. E.; Lewis, C. L.; Majidi, V. J. Anal. At. Spectrom. 1999, 14, 15371541.

(34) Majidi, V.; Moser, M.; Lewis, C.; Hang, W.; King, F. L. J. Anal. At. Spectrom. 2000, 15, 19-25.

(35) Coburn, J. W.; Kay, E. Appl. Phys. Lett. 1971, 18, 435-438.

(36) Smith, R. L.; Serxner, D.; Hess, K. R. Anal. Chem. 1989, 61, 1103-1108.

(37) Hess, K. R.; Harrison, W. W. Anal. Chem. 1988, 60, 691-696.

(38) Blair, S. M.; Brodbelt, J. S.; Marchand, A. P.; Kumar, K. A.; Chong, S. Anal. Chem. 2000, 72, 2433-2445.

(39) Imura, K.; Midorikawa, R.; Kasai, T.; Ohoyama, H.; Che, D. Chem. Lett. 1996, 299-300.

(40) Velazco, J. E.; Kolts, J. H.; Setser, D. W. J. Chem. Phys. 1978, 69, 4357-4373.

(41) Copley, G. H.; Lee, C. S. Can. J. Phys. 1975, 53, 1705-1714.

(42) Ferreira, C. M.; Loureiro, J.; Ricard, A. J. Appl. Phys. 1985, 57, 82-90.

(43) Hoppstock, K.; Harrison, W. W. Anal. Chem. 1995, 67, 3167-3171.

(44) Bogaerts, A.; Gijbels, R. Spectrochim. Acta, Part B 2000, 55, 263-278.

(45) Lewis, C. L.; Jackson, G. P.; Doorn, S. K.; Majidi, V.; King, F. L. Spectrochim. Acta, Part B 2001, 56, 487-501.

(46) Wiese, W. L.; Brault, J. W.; Danzmann, K.; Helbig, V.; Kock, M. Phys. Rev. A 1989, 39, 2461-2471.

(47) Jansson, P. A. Deconvolution: with applications in spectroscopy; Academic Press, Inc.: Orlando Fl, 1984. 


\section{Chapter 3}

\section{Bulk Plasma Properties in the Pulsed Glow Discharge}

\subsection{Introduction}

Glow Discharge Mass Spectrometry (GDMS) is a versatile, simple, and robust technique for the analysis of conducting and non-conducting solids. ${ }^{1-7}$ For analytical applications, research has shown that pulsing the glow discharge source in the micromillisecond time frame offers many advantages over steady state discharges. ${ }^{8-13}$ These benefits are gained, in part, because higher instantaneous powers can be used in the pulsed mode without fear of cracking or melting the sample cathode. The cathode has time to cool between pulses and this alleviates the necessity for water-cooled probes and flanges. Increased power leads to increases in sputtering and excitation/ionization of the sputtered species. This, in turn, affords greater signal-to-noise ratios and lower limits of detection for analytical techniques employing pulsed glow discharge sources (PGDs). ${ }^{10}$

In order to fully exploit the advantages of PGDs, a fundamental understanding of the excitation and ionization phenomena occurring throughout the various temporal and spatial domains of the plasma is required. With knowledge of the underlying mechanisms of energy exchange within the plasma, one is more aptly positioned to devise experiments that would further utilize the many advantages of PGDs. ${ }^{14}$ This Chapter focuses on the plasma evolution over a typical pulse cycle and how these changes affect analyte signals, with emphasis devoted to the effects on analyte emission signals. 
The spatial and temporal characteristics of metastable argon atoms and sputtered copper species are presented in Chapter 2 and elswhere. ${ }^{15-17}$ Their behavior does not necessarily give an indication of the nature of the discharge as a whole because these species account for only a small fraction of the total plasma composition. Therefore, to understand the bulk energetics of the plasma, knowledge of the spatio-temporal fluctuations in excitation and ionization for the discharge gas (argon) is required.

Rózsa and Gallagher ${ }^{18}$ studied the spatial dependence of emission from argon atoms and ions in a constant current glow discharge source operating at $\sim 0.2$ Torr. The low pressure used (relative to most analytical glow discharges that operate at $\sim 1.0$ Torr or greater) clearly separated the negative glow from the cathode darkspace, enabling comparisons to be made between the two regions. Such comparisons are more difficult at higher pressures because the two regions contract closer to the cathode. Rózsa and Gallagher ${ }^{18}$ note that the excitation mechanisms that dominate these two regions are very different. Heavy particle bombardment dominates the cathode darkspace whereas electrons dictate the excitation in the negative glow. Bogaerts and Gijbels developed an emission model for argon in a steady state glow discharge operating at 5.0 Torr. ${ }^{19-23}$ Their work focused primarily on electron excitation mechanisms because the negative glow dominates the plasma at higher pressures. This predictive capability, and other models, ${ }^{24-}$ 28 demonstrate that the fundamental processes occurring in steady-state GDs are reasonably well known.

The same level of understanding has not yet been realized for pulsed glow discharges $^{29}, 30$ although considerable efforts have been made to characterize their emission and ionization characteristics. Duncen, et al. ${ }^{31}$ observed an increase in emission 
from argon atoms following power termination in a pulsed glow discharge and suspected that contaminated gases played some role in this process. Strauss, et al. ${ }^{32}$ reported that when the voltage is suddenly removed from a steady-state discharge, the emission intensity of selected wavelengths suddenly increased to nearly double that of the steady state intensity. These afterglow ${ }^{\mathrm{a}}$ emissions could be reduced by the addition of a quenching agent (methane). Although the afterglow emissions were attributed to recombination, the exact mechanism for methane quenching of this effect was not identified. Evidence was provided, however, that the metastable states of argon were greatly reduced in the afterglow by the addition of the methane, indicating that perhaps the metastables were involved in the afterglow process. Strauss and coworkers did not observe any variation in the shape of the decaying emission or absorption curves as a function of distance. ${ }^{32}$ However, their observations were made at 1.8 Torr and at distances beyond $2.0 \mathrm{~mm}$. It is likely that their observations were made entirely in the negative glow where similar energy conversion mechanisms are found throughout.

Like any other plasma, the GD plasma mainly consists of ground state argon atoms, excited state neutrals (including metastable states), and ground- and excited state argon ions of various charge states, electrons and photons. On a microscopic level, these species are in a constant state of flux as energy is continually exchanged among them through various interactions. If every process that occurs in the plasma is fully reversible, the plasma is said to be in a state of thermodynamic equilibrium (TE) and the description of the plasma is relatively simple. ${ }^{33}$ The macroscopic state of the plasma can be fully

\footnotetext{
${ }^{a}$ The terms afterglow and afterpeak are used interchangeably throughout this chapter. Both terms refer to the observation of a given signal after the termination of the plasma-sustaining voltage. Emission signals are often found to go through a maximum in the afterglow and the value at this maxim is more accurately called the afterpeak signal.
} 
described by various statistical models that govern the distribution of energy levels in the plasma. In TE, the equations of Maxwell, Boltzmann, Saha, and Planck fully describe the velocity distribution, the excited state distribution, the degree of ionization, and the transfer of photons, respectively. Significantly, all of these processes are balanced permitting the energy for a plasma in TE to be defined by a single temperature. If, for any reason, a given process ceases to be perfectly reversible (for example, if particles are lost to the chamber walls), the plasma will cease to be in equilibrium and it's statistical description becomes much more difficult. ${ }^{33-38}$

PGDs do not display $\mathrm{TE}^{39}$ because the proper forward and reverse reactions do not always balance. For example, ions diffuse to the chamber walls and initially disrupt the Saha balance. This deviation from equilibrium consequently affects the Boltzmann and Planck distributions because excited state neutrals are no longer formed by recombination reactions. Because of the various ways in which plasmas can deviate from ideality, a more detailed understanding of the balances and relations that do occur in PGDs is necessary to understand how they behave. Such an understanding is useful in determining how they can be harnessed for greater analytical benefit.

\subsection{Experimental}

The experimental apparatus used in this study is described in detail in Chapter 2

and elsewhere. ${ }^{40}$ A stainless steel, six-way cross housed the direct insertion probe onto which sample cathode disks were mounted. A copper disk (NIST SRM 495, Gaithersburg, MD) of $4.0 \mathrm{~mm}$ diameter was used as the cathode. A translation platform held the six-way cross and could be moved orthogonal (in the x-dimension) to the 
monochromator to permit observations of different regions of the plasma. The direct insertion probe also maneuvered orthogonal to the monochromator (in the $y$-dimension to alter the sampling distance above the cathode. In this way two-dimensional emission maps could be obtained for each line.

Operating conditions were as follows: 0.8 Torr argon discharge gas, $1.6 \mathrm{~mA}$ peak current, $\sim 860 \mathrm{~V}$ applied voltage. The emission from the discharge cell was imaged in a 1:1 ratio on the entrance slit of the monochromator. Entrance and exit slits were $50 \mu \mathrm{m}$ wide and $1.0 \mathrm{~mm}$ high. The extended-red photomultiplier tube (R-928, Hamamatsu, Bridgewater, NJ) operated at $800 \mathrm{~V}$, unless otherwise stated.

\subsection{Results and Discussion}

\subsubsection{Argon atom emission}

Figures 3.1.a and 3.1.b show energy level diagrams for the selected argon atom and ion states, respectively. The individual emission lines studied are given in Tables 3.1 and 3.2. The argon atom lines are classified into groups according to the manifolds from which they originate. These groups are labeled as at on the energy level diagram in Figure 3.1. Emission spectra (not shown) follow the general trend observed by most groups, i.e. the "red" lines due to $5 \mathrm{p}-4 \mathrm{~s}$ transitions are most intense while the "blue" lines, responsible for the characteristic blue glow in the glow discharge, are less intense. 


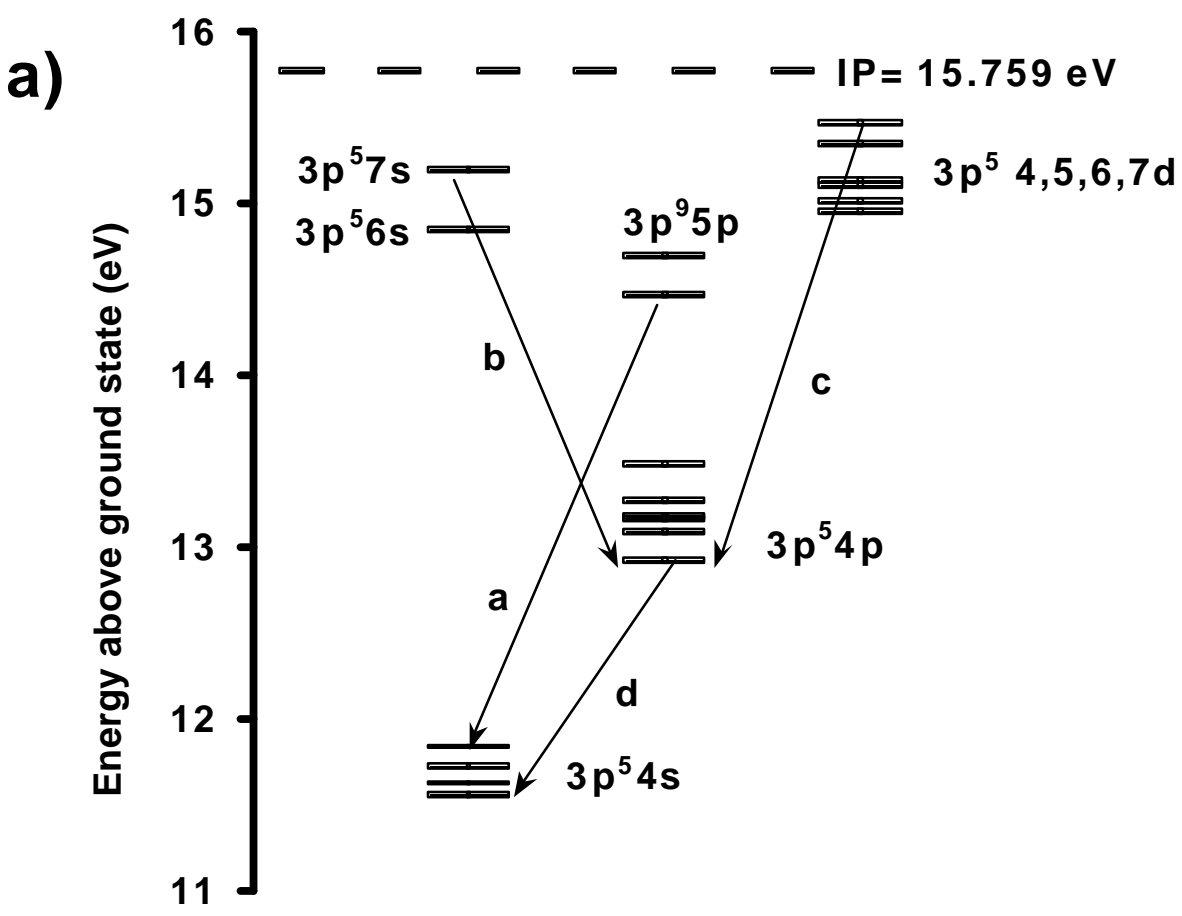

b)

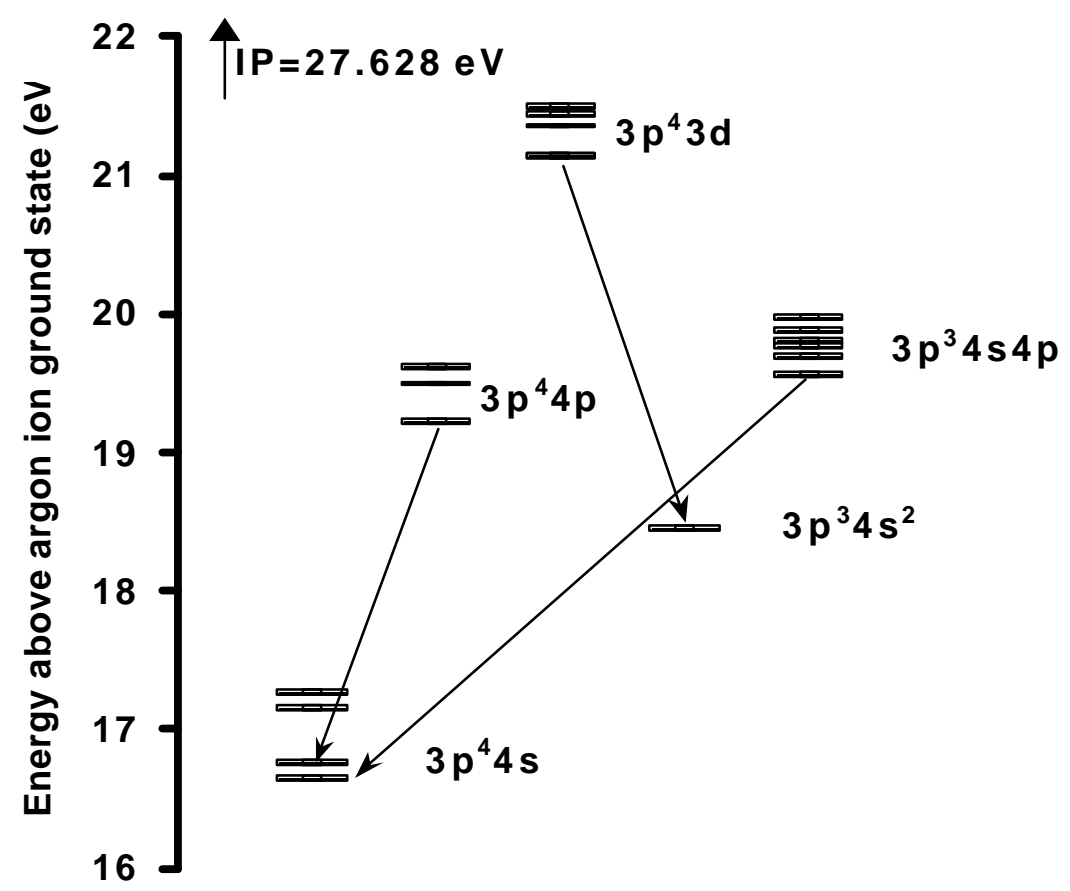

Fig. 3.1. Energy level diagrams showing the lines of interest for the various states of the argon atom (a) and argon ion (b). 
Table 3.1. Spectroscopic data for argon atom lines examined in this study.

\begin{tabular}{|c|c|c|c|c|c|}
\hline \multirow{2}{*}{$\begin{array}{c}\text { Wavelength } \\
\left(\mathbf{A}^{0}\right)\end{array}$} & \multirow{2}{*}{$\begin{array}{c}\text { Transition } \\
\text { probability } \\
A\left(1^{8} \mathrm{~s}^{-1}\right)^{41}\end{array}$} & \multicolumn{2}{|c|}{ Energy Levels (eV) } & \multicolumn{2}{|c|}{$\begin{array}{c}\text { Statistical } \\
\text { Weights }\end{array}$} \\
\hline & & $\mathbf{E}_{\mathbf{L}}$ & $\mathbf{E}_{\mathbf{U}}$ & $\mathbf{g}_{\mathrm{L}}$ & $\mathbf{g}_{\mathbf{U}}$ \\
\hline $4 \quad 4200.7$ & $\begin{array}{l}0.010 \\
\end{array}$ & $\begin{array}{c}11.55 \\
\end{array}$ & $\begin{array}{l}14.46 \\
\end{array}$ & 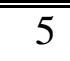 & 87 \\
\hline 4333.6 & 0.006 & 11.83 & 14.69 & 3 & 5 \\
\hline 5373.5 & 0.003 & 13.15 & 15.46 & 3 & 5 \\
\hline 5495.9 & 0.017 & 13.08 & 15.33 & 7 & 9 \\
\hline 5650.7 & 0.032 & 12.91 & 15.10 & 3 & 1 \\
\hline 5888.6 & 0.013 & 13.08 & 15.18 & 7 & 5 \\
\hline 5912.1 & 0.011 & 12.91 & 15.00 & 3 & 3 \\
\hline 6664.1 & 0.002 & 13.09 & 14.95 & 5 & 5 \\
\hline 6719.2 & 0.002 & 13.27 & 15.12 & 1 & 3 \\
\hline 7503.9 & 0.445 & 11.83 & 13.48 & 3 & 1 \\
\hline 7514.7 & 0.402 & 11.62 & 13.27 & 3 & 1 \\
\hline 7635.1 & 0.245 & 11.55 & 13.17 & 5 & 5 \\
\hline 7723.8 & 0.052 & 11.55 & 13.15 & 5 & 3 \\
\hline 8115.3 & 0.331 & 11.55 & 13.08 & 5 & 7 \\
\hline
\end{tabular}

$E_{l}$ and $E_{u}$ refer to the lower and upper energy levels of the states, respectively. $g_{a}$ and $g_{u}$ refer to the lower and upper statistical weights, respectively. 
Table 3.2. Spectroscopic data for argon ion lines examined in this study

\begin{tabular}{c|c|c|c|c|c|}
\multirow{2}{*}{$\begin{array}{c}\text { Wavelength } \\
\left.\mathbf{( A}^{\mathbf{0}}\right)\end{array}$} & $\begin{array}{c}\text { Tranistion } \\
\text { probability }\end{array}$ & \multicolumn{2}{|c|}{ Energy Levels (eV) } & \multicolumn{2}{|c|}{$\begin{array}{c}\text { Statistical } \\
\text { Weights }\end{array}$} \\
\cline { 3 - 6 }$\left(\mathbf{( 1 0}^{\mathbf{8}} \mathbf{s}^{\mathbf{- 1}}\right)^{\mathbf{4 1}}$ & $\mathbf{E}_{\mathbf{L}}$ & $\mathbf{E}_{\mathbf{U}}$ & $\mathbf{g}_{\mathbf{L}}$ & $\mathbf{g}_{\mathbf{U}}$ \\
\hline \hline 4131.7 & 1.4 & 18.43 & 21.43 & 4 & 2 \\
4277.5 & 0.41 & 18.45 & 21.35 & 4 & 6 \\
4331.2 & 0.56 & 16.75 & 19.61 & 4 & 4 \\
4348.1 & 1.24 & 16.64 & 19.49 & 6 & 8 \\
4370.8 & 0.65 & 18.66 & 21.49 & 4 & 4 \\
4401.0 & 0.322 & 16.41 & 19.22 & 8 & 6 \\
4426.0 & 0.83 & 16.75 & 19.55 & 4 & 6 \\
4545.1 & 0.413 & 17.14 & 19.87 & 4 & 4 \\
4590.0 & 0.82 & 18.43 & 21.13 & 4 & 6 \\
4609.6 & 0.91 & 18.45 & 21.14 & 6 & 8 \\
4657.9 & 0.81 & 17.14 & 19.80 & 4 & 2 \\
4726.9 & 0.5 & 17.14 & 19.76 & 4 & 4 \\
4764.9 & 0.575 & 17.26 & 19.87 & 2 & 4 \\
4806.0 & 0.79 & 16.64 & 19.22 & 6 & 6 \\
4879.9 & 0.78 & 17.14 & 19.68 & 4 & 6 \\
4965.1 & 0.347 & 17.26 & 19.76 & 2 & 4
\end{tabular}


Figure 3.2 shows the temporal emission profiles for four selected argon emission lines. Each line presented is representative of the general behavior observed for the other transitions between the manifolds shown in Figure 3.1.a. The effects of sampling height are readily discernable in Figure 3.2. That the emission characteristics vary so considerably at different heights demonstrates the inhomogeneity of the plasma and the necessity for making diagnostic measurements parallel to the cathode surface, 'side-on', instead of the 'end-on' approach used for conventional analyses.

\subsubsection{1. $3 p^{5} 5 p-3 p^{5} 4 s$ transitions (Figures 3.2.a and 3.3.a)}

The "blue" lines result in the population of 4 possible states. Two of these states, $\left({ }^{1} \mathrm{~s}_{2}\right){ }^{1} \mathrm{P}_{1}$ and $\left({ }^{1} \mathrm{~s}_{4}\right){ }^{3} \mathrm{P}_{1}$, are resonant with the ground state and are too high in frequency to be observed with the apparatus employed. Transitions between the remaining states, $\left({ }^{1} s_{3}\right)$ ${ }^{3} \mathrm{P}_{0}$ and $\left({ }^{1} \mathrm{~s}_{5}\right){ }^{3} \mathrm{P}_{2}$, and the ground state are spin forbidden, and therefore metastable. If unperturbed, they may exist for several seconds. ${ }^{42}$ At $\sim 1$ Torr, their lifetime is more on the order of 1-10 ms. ${ }^{43,44}$ Under the operating conditions used here, approximately 30$50 \%$ of the metastable argon atoms are reported to form via radiative decay from the $5 p$

level. ${ }^{20}$ For this reason, the emission characteristics of this array give a good indication of the location of these metastable atom populations. 

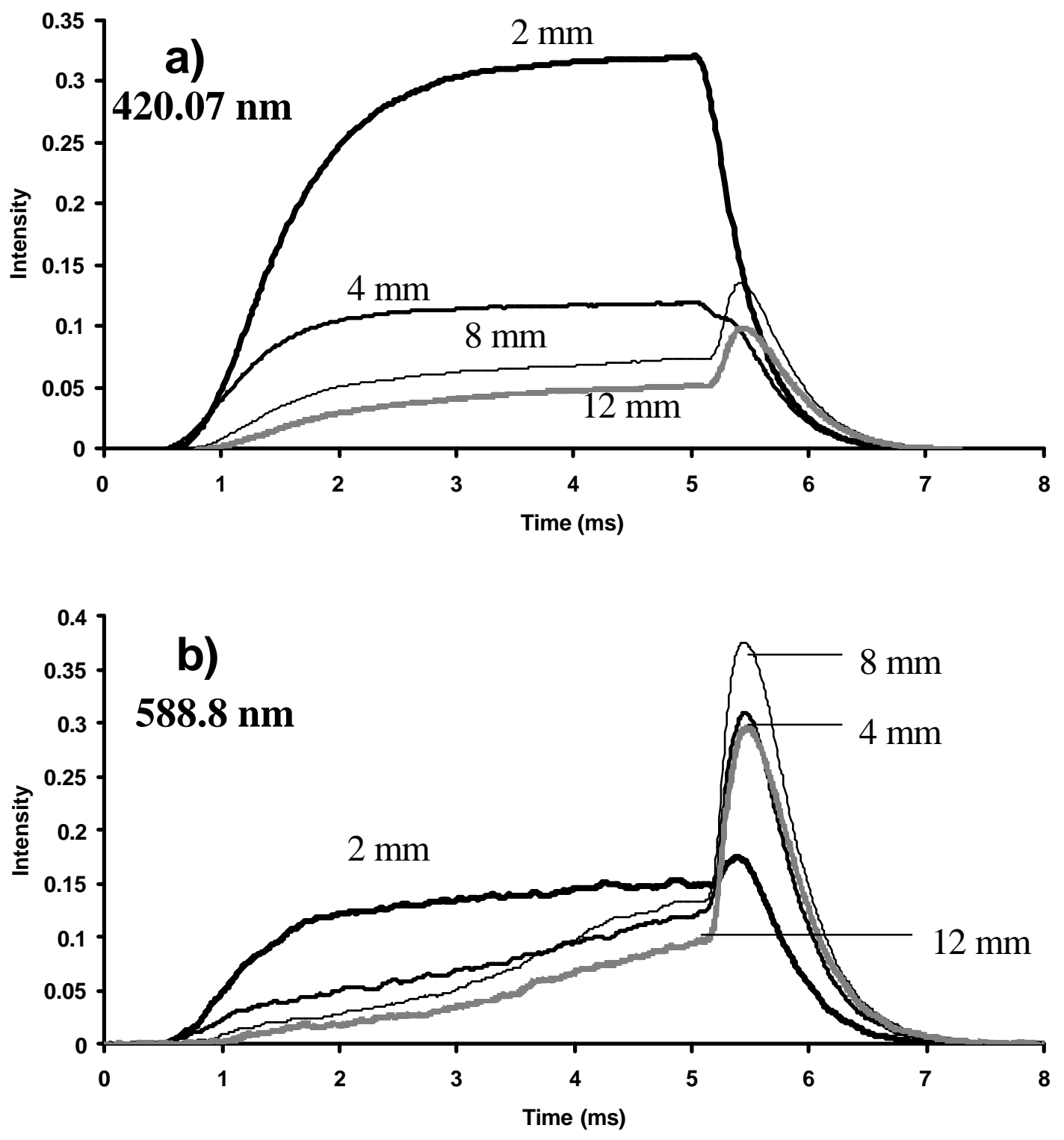

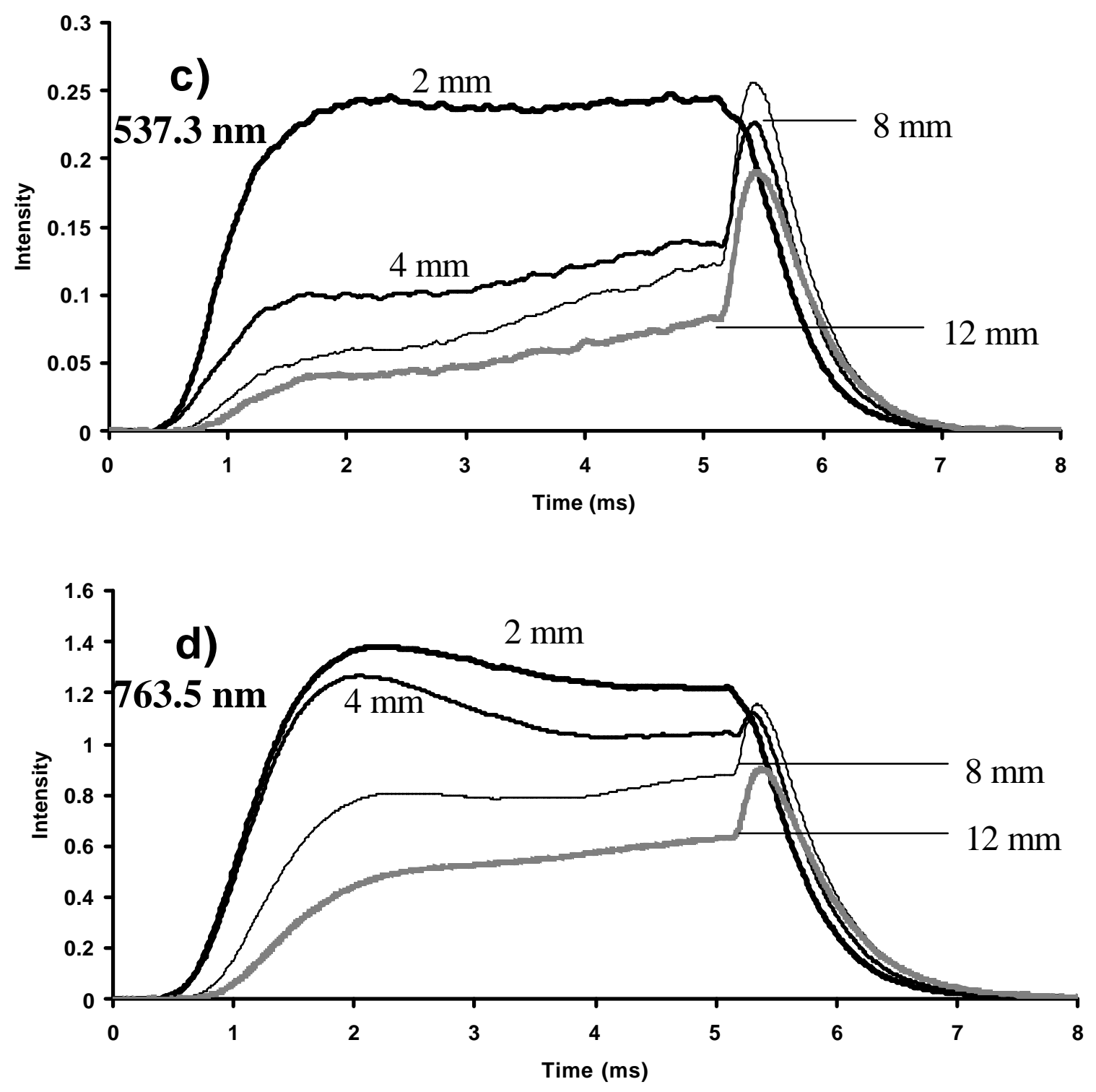

Fig. 3.2. Temporal traces for selected argon atom transitions at different distances above the cathode. a), b), c), and d) refer to the labels in Figure 3.1.a. Pulse length 5.0 ms, 0.8 Torr Ar, $1.5 \mathrm{~W}$ peak power. 

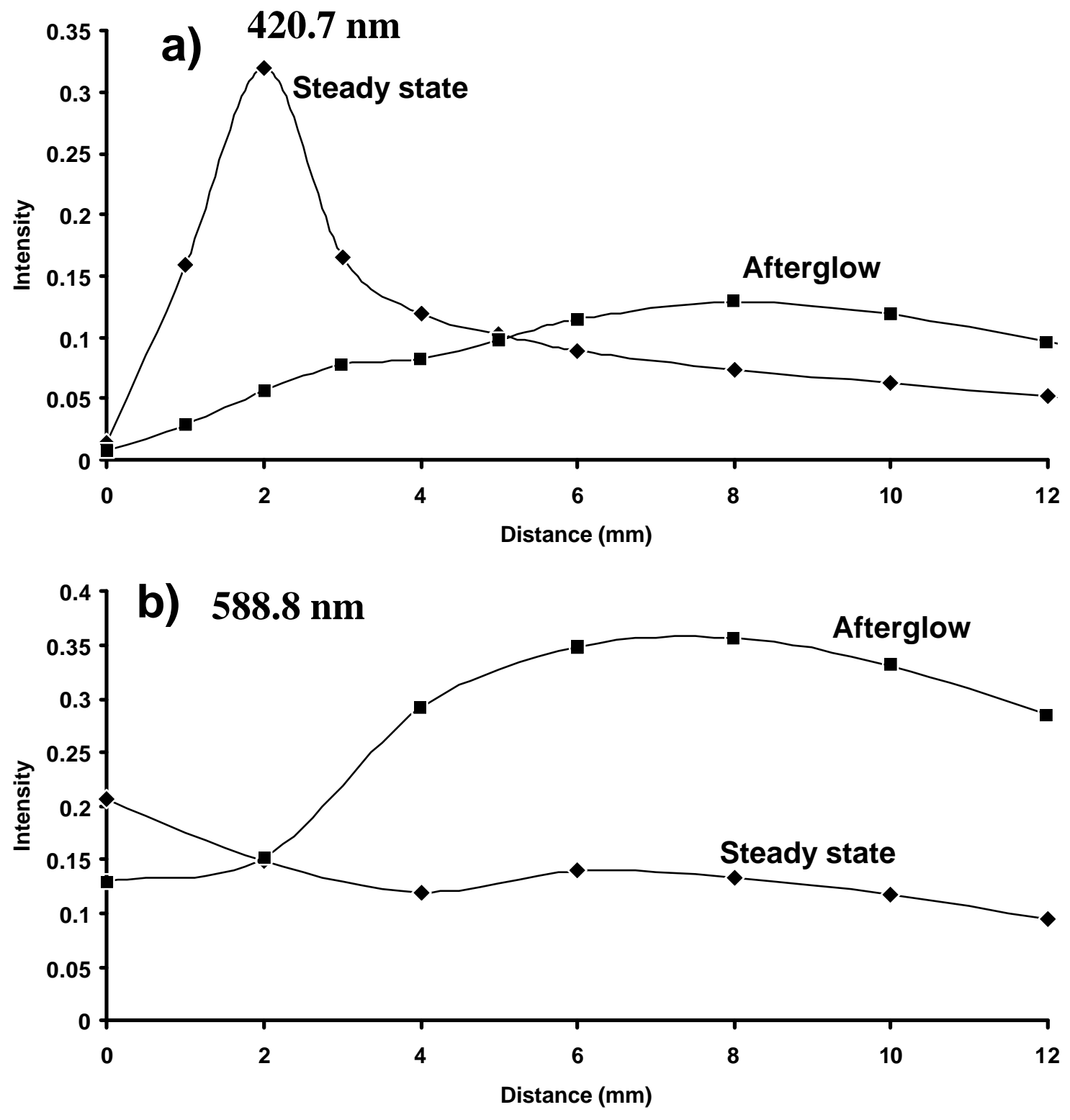

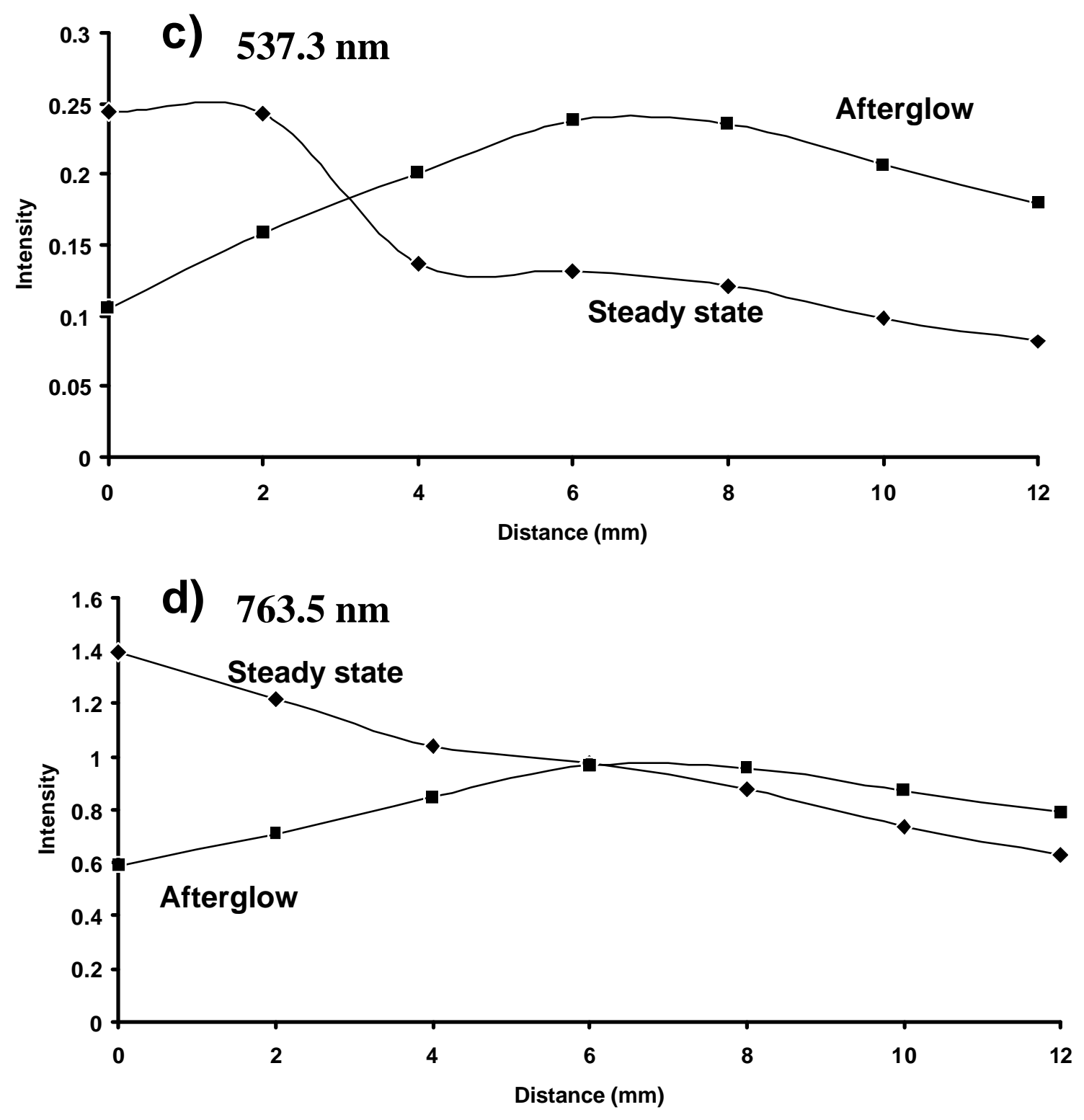

Fig. 3.3. Optical emission signals for argon atom lines in the steady-state (diamonds) and afterglow (squares) as a function of distance. a), b), c), and d) refer to the labels in Figure 3.1.a. Pulse length $5.0 \mathrm{~ms}, 0.8$ Torr Ar, $1.5 \mathrm{~W}$ peak power. 
The temporal profiles of emission intensity at $420.67 \mathrm{~nm}\left(5 \mathrm{p}\left[2^{1 / 2}\right]-4 \mathrm{~s}\left[1 \frac{1}{2}\right], 14.50-\right.$ $11.55 \mathrm{eV}$ ) are plotted in Figure 3.2.a for various observation points. The emission intensity as a function of distance is presented in Figure 3.3.a. The maximum emission intensity over the entire pulse cycle is found at $2.0 \mathrm{~mm}$ above the cathode during power application. The maximum emission intensity during the voltage decreases dramatically from 2.0-4.0 $\mathrm{mm}$, and decreases in linear fashion at greater distances. Comparable behavior was observed by Rózsa and Gallagher for a similar transition. ${ }^{18}$ The behavior is attributed to the method of $5 \mathrm{p}$ level population: close to the cathode, excitation is by fast atom bombardment; whereas, in the negative glow it is predominantly by electron excitation. Upon power termination, little or no afterpeak is observed between 0-2 $\mathrm{mm}$. The lack of an afterpeak close to the cathode can be attributed to one (or both) of the following possibilities. i) Electrons do not play a significant role in populating these states while the discharge is on. If electrons populated these levels, the sudden decrease in electron temperature in the afterpeak would be expected to cause a 'Saha-jump' in the afterglow. ${ }^{45-47}$ ii) electrons are not available for recombination in the afterglow because they are lost to the cathode surface.

When the plasma is extinguished a surge in the emission intensity is observed for this line at distances beyond $2.0 \mathrm{~mm}$. Figure 3.3.a shows that the afterpeak intensity maximizes at $\sim 6 \mathrm{~mm}$ at this pressure. The emission maximum in the afterglow appears at 200-300 $\mu$ s under these conditions. A pulsed power supply with a faster fall time (not available for all the experiments provided here) provided afterpeak maxima in the region of $100-200 \mu$ s. The time delay between voltage termination and the observed emissions is related to the time required for the fast electrons to thermalize via collisions. ${ }^{48,49}$ This is 
thought to occur very rapidly at pressures of $\sim 10-760$ Torr- on the order of $1 \mu$ s or less, ${ }^{48,50}$ but takes longer-on the order of $100 \mu \mathrm{s}$ - at lower pressures. ${ }^{51}$ Recombination is also more probable further from the cathode because the argon ion population, which is predominantly formed in the negative glow, is largest between $4-8 \mathrm{~mm}{ }^{52,}{ }^{53}$ The afterpeak maximum shifted to slightly longer times ( by $10-20 \mu \mathrm{s}$ ) at $16-20 \mathrm{~mm}$ from the cathode, indicating that the electron temperature may take slightly longer to decrease at these long distances. The emission decay rates, occurring after the afterpeak maximum, are discussed in more detail in Section 3.3.6. The emission maximum in the afterglow appears at 200-300 $\mu$ s under these conditions. A pulsed power supply with a faster fall time (not available for all the experiments provided here) provided afterpeak maxima in the region of $100-200 \mu$ s. The time delay between voltage termination and the observed emissions is related to the time required for the fast electrons to thermalize via collisions. ${ }^{48,49}$ Thermalization will obviously take longer to occur if the applied voltage is not quickly removed, and continues to accelerate electrons for a short time in the afterglow.

\subsubsection{2. $3 p^{5} 7 s-3 p^{5} 4 p$ transitions (Figures 3.2.b and 3.3.b)}

Figure 3.3.b presents the emission intensity of the $7 \mathrm{~s}$ level at $588.86 \mathrm{~nm}\left(7 \mathrm{~s}\left[1 \frac{1}{2}\right]-\right.$ $4 \mathrm{p}\left[2 \frac{1}{2}\right], 15.18-13.08 \mathrm{eV}$ ) during power application (diamonds) and in the afterglow (squares). Power application generates maxima in the populations at two spatial positions. One maximum is at the cathode surface, where fast atom/ion bombardment is the dominant excitation mechanism. ${ }^{18,25,54}$ The other maximum is found at $\sim 6 \mathrm{~mm}$ above the cathode where electron excitation dominates. Relative to the emission at $420.67 \mathrm{~nm}$, 
the cathode darkspace/negative glow intensity ratio is much smaller for this transition. This indicates the smaller probability of accessing this higher energy $7 \mathrm{~s}$ state via fast atom/ion collisions than the lower energy $4 \mathrm{p}$ states.

In a recent model of a steady state GD proposed by Bogaerts et. Al., ${ }^{20,55}$ it is assumed that fast atom/ion bombardment is not capable of exciting argon atoms to the $7 \mathrm{~s}$ state. Therefore, in the model, the only maximum is predicted to occur is in the negative glow. Such subtle differences between the model and experiments are to be expected given the complex nature of the plasma and the differences in conditions studied.

It can be seen from Figure 3.2.b that the $7 \mathrm{~s}-4 \mathrm{p}$ transition reaches a steady state value close to the cathode surface but not at distances beyond $2.0 \mathrm{~mm}$ from the surface. Because all the transitions involving an upper energy level of $15 \mathrm{eV}$ or higher showed this behavior, one might expect that a stepwise mechanism is responsible for populating these high levels. Indeed, Bogaerts et al. report that the electron excitation from alreadyexcited levels increases in importance for populating these higher levels. ${ }^{20,55}$ At $5.0 \mathrm{~ms}$ the intensity reaches a maximum in the negative glow at $\sim 6 \mathrm{~mm}$. Bogaerts et al. predict this population to occur $\sim 2 \mathrm{~mm}$ above the cathode in a 1.0 Torr plasma. ${ }^{55}$ The present observation, at 0.8 Torr, is reasonably consistent with the model when the pressure difference is taken into account.

Within $2.0 \mathrm{~mm}$ of the cathode, the emission intensity at $588.86 \mathrm{~nm}$ decays rapidly upon power termination. No afterpeak is evident (for reasons discussed in section 3.3.1.1). Further from the cathode, however, a significant increase in afterpeak emission is observed (see Figure 3.2.b). The maximum intensity of the afterpeak is also found 
between 6-8 $\mathrm{mm}$. The afterpeak/plateau emission intensity ratio is much larger for this transition than for the $5 \mathrm{p}-4 \mathrm{~s}$ transitions.

\subsubsection{3. $3 p^{5} 4-7 d-3 p^{5} 4 p$ transitions (Figures 3.2.c and 3.3.c)}

The transitions of this manifold are typified by the emission at $537.35 \mathrm{~nm}$ $\left(7 \mathrm{~d}\left[2^{1 / 2}\right]-4 \mathrm{p}\left[\frac{1}{2}\right], 15.46-13.15 \mathrm{eV}\right)$. The only significant difference between the emissions from the $4 \mathrm{~d}-7 \mathrm{~d}$ states is that the afterglow/steady-state emission ratio is larger for the emissions originating from the higher levels. In Figure 3.3.c it can be seen that during power application the emission intensity maximizes in the negative glow. A smaller maximum is observed at the cathode surface, indicating that fast atom/ion bombardment is a less significant mechanism of populating these levels. Indeed, studies show that higher energy electronic orbitals have a smaller probability of being formed via fast atom/ion bombardment. ${ }^{54}$ Although the $7 \mathrm{~d}$ state has a slightly lower energy than the $7 \mathrm{~s}$ state (mentioned in the previous section) the cathode darkspace/negative glow emission intensity for the $7 \mathrm{~d}$ state is considerably smaller. It is possible that the energy level alone may not be an accurate guide as to the probability of being formed by fast atom/ion bombardment and that other selection rules are involved.

\subsubsection{4. $3 p^{5} 4 p-3 p^{5} 4 s$ transitions (Figures 3.2.d and 3.3.d)}

Often classified as the "red" lines, these transitions are typified by the emission at $763.51 \mathrm{~nm}\left(4 \mathrm{p}\left[1 \frac{1}{2}\right]-4 \mathrm{~s}\left[1 \frac{1 / 2}{2}\right], 13.17-11.55 \mathrm{eV}\right)$. All of the emission lines observed between these levels behave in a very similar fashion. Temporal profiles between 2.0-8.0 $\mathrm{mm}$, as seen in Figure 3.2.d, display a distinct pre-peak at $\sim 2 \mathrm{~ms}$. A pre-peak is defined as 
an emission signal between 0-2.5 ms that is larger than the steady-state signal between 4$5 \mathrm{~ms}$. Because the onset of the pulsed discharge commences with a cascade of electrons, it appears that the $4 \mathrm{p}$ levels are preferentially formed by electron excitation mechanisms than other states. Indeed, no prepeak behavior is observed from the $5 \mathrm{p}$ levels and they are the next closest in energy to the $4 p$ states.

The maximum intensity at $5.0 \mathrm{~ms}$ is found at the cathode surface and the intensity decays almost linearly as a function of distance from the cathode. This is shown in Figure 3.3.d. In this case the cathode darkspace and negative glow populations are not as clearly separable as for the other lines. The afterpeak emission intensities, also shown in Figure $3 \mathrm{~d}$, increase gradually from a minimum at the cathode surface to a maximum between 6-8 $\mathrm{mm}$. Only at distances beyond $6 \mathrm{~mm}$ does the afterpeak intensity exceed that of the plateau. The noticeable difference in plateau and afterpeak emission intensities for this line as compared to the previous three lines illustrates the dramatic differences the emission profiles can assume depending on upper electronic energy level of the emitting species.

\subsubsection{Deviations from thermodynamic equilibrium}

All the atom lines observed in this study displayed an afterpeak to some degree (at distances beyond $2 \mathrm{~mm}$ above the cathode surface). The observation of this surge in emission intensity for all argon atom species can be understood in terms of the plasma shifting from an ionizing plasma during power application to a recombining plasma in the afterpeak. Evidence of such behavior has been theoretically described ${ }^{34-37}$ and

experimentally demonstrated. ${ }^{56}$ Because these phases deviate from the ideal balances 
predicted by thermodynamic equilibrium (TE), the characteristics of non-TE plasmas will be described in more detail in the following sections.

\subsubsection{Departure from the Maxwell balance}

As mentioned in the introduction, the Maxwell balance describes the velocity distribution of particles in the plasma. This description includes atoms, ions and electrons and is represented by the exchange of kinetic energy between particles $\mathrm{X}$ and $\mathrm{Y}$ in Equations 3.1 and 3.2.

$$
\begin{gathered}
X+Y \leftrightarrow X^{\prime}+Y^{\prime} \\
E_{X^{\prime}}+E_{Y^{\prime}}=\left(E_{X}+\Delta E\right)+\left(E_{Y}-\Delta E\right)
\end{gathered}
$$

The collisions are purely elastic, obey the law of conservation of momentum ${ }^{57}$ and the internal energies of $\mathrm{X}$ and $\mathrm{Y}$ remain unchanged. The distribution of states (velocities), $f(v)$, for a given species is proportional to ${ }^{57}$

$$
f(v) \propto \mathrm{e}^{-\mathrm{E} / \mathrm{RT}}
$$

where $\mathrm{E}$ is the kinetic energy $\left(=1 / 2 \mathrm{M} v^{2}\right.$, where $\mathrm{M}$ and $v$ are the mass and velocity of the species), $\mathrm{R}$ is the gas constant and $\mathrm{T}$ is the temperature. If one assumes that $\mathrm{TE}$ is established between all the particles in the plasma, i.e. the temperature of the electrons equals that of the atoms and ions in the plasma, one temperature can be used to calculate the velocity distribution of any species. Unfortunately, for reasons discussed below, glow discharges do not obey this vital requirement.

Energy is deposited into GDs by an electric field—most commonly by a dc field. The electric field accelerates charged particles in preference to neutral particles, so the very nature of energy deposition in the plasma is non-uniform. The plasma must 
redistribute the kinetic energy of the charged species with the neutral species if it is to obey a Maxwellian distribution. This transfer of energy into the plasma can be presented by the following energy flow diagram. ${ }^{45,47}$

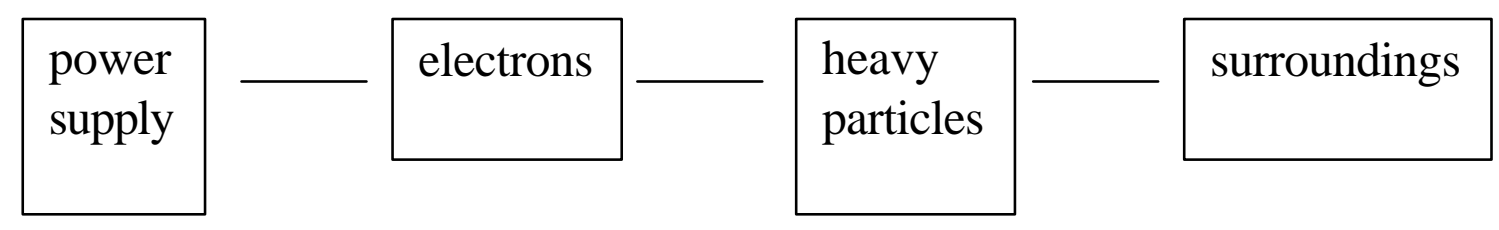

The power supply deposits energy into the electrons and they, in turn, heat up the heavy particles. This is a relatively inefficient process because the particles are so dissimilar in mass. ${ }^{57}$ Therefore, electrons accelerated by the electric field more-readily redistribute their kinetic energy with other electrons in the negative glow than with atoms or ions. Following a similar argument, the gas atoms and ions more readily distribute their energy among one another than with electrons. Because the discharge chamber is relatively small $(2-8 \mathrm{~cm}$ in diameter in most GD sources), the heavy particles tend to be permanently cooled by collisions with the walls. For these reasons, the electrons have a disparate kinetic temperature to the atoms and ions in the discharge. This is one cause for departure from TE in the plasma and it originates from the method by which energy is deposited in the plasma.

If this was the only cause for departure from TE the plasma could be called a twotemperature (2T) plasma. ${ }^{33}$ In $2 \mathrm{~T}$ plasmas it is generally recognized that the electrons' velocity distribution is defined by one temperature, $T_{e}$, while the 'heavy' gas particles (atoms and ions) velocities are defined by a second temperature, $\mathrm{T}_{\mathrm{g} .}{ }^{33}$ The atomic-state distribution function is then a competition between the Boltzmann and Saha balances. 
The Boltzmann balance describes the distribution of excited states formed via electron excitation from the ground state and is represented by

$$
\mathrm{X}^{0}+\mathrm{e}_{\text {fast }}^{-} \leftrightarrow \mathrm{X}^{\mathrm{p}}+\mathrm{e}_{\text {slow }}^{-}
$$

Where $\mathrm{X}^{0}$ is the ground state and $\mathrm{X}^{\mathrm{p}}$ is an excited state of $\mathrm{X}$ in quantum level $\mathrm{p}$. As the number of fast electrons increases, so does the number density of excited states of $\mathrm{X}^{\mathrm{p}}$. In the Saha balance

$$
\mathrm{X}^{\mathrm{p}}+\mathrm{e}_{\text {fast }}^{-} \leftrightarrow \mathrm{X}^{+}+\mathrm{e}_{\text {slow }}^{-}+\mathrm{e}_{\text {slow }}^{-}
$$

the population of excited neutral states is determined by the number density of the ground state ions and by the electron temperature. In the Saha balance, an increase in the number of fast electrons will decrease the number of excited states of $\mathrm{X}^{\mathrm{p}}$. The two balances both involve electrons and are consequently $\mathrm{T}_{\mathrm{e}}$ dependant. If the degree of ionization is large and charge species are not lost to the surroundings the Saha balance will prevail. ${ }^{33}$ If the degree of ionization is not large, the Boltzmann balance will govern the lower levels of the steady-state distribution and the Saha balance will dominate the higher levels. The following discussion determines the dominant populating mechanisms in the PGD, and whether the 2T principle is relevant to GD's or PGDs.

\subsubsection{Departure from the Saha-Boltzmann balance}

A relatively simple method has been devised ${ }^{46,47}$ to determine the departure of a plasma from TE conditions, and to ascertain the values of $T_{e}$ and $T_{g}$ in the process (if reasonable TE exists) This technique utilizes time-dependant intensities of spectral lines emitted by neutral atoms in response to the removal of the electric field. There are several assumptions that must be satisfied in order for this approach to be successful: i) the 
plasma must be at a steady state before the voltage is removed, ii) the electron relaxation time must be considerably faster than the recombination time, and iii) the plasma must be optically thin (meaning that light escapes fairly readily and is not re-absorbed by the plasma). It has been shown that $5 \mathrm{~ms}$ is usually adequate time to achieve a steady state condition, ${ }^{16,40,58-60}$ and that the GD configuration and operating conditions used here provide an optically thin plasma. ${ }^{39}$

If an energy level is populated predominantly by the Saha balance during power application, the response to power termination will be $\mathrm{e}^{46,47}$

$$
\ln \left(I^{\prime} / \mathrm{I}\right)=3 / 2 \ln \gamma+\left(\mathrm{E}_{\text {ion }}-\mathrm{E}_{\mathrm{p}}\right)\left([\gamma-1] / \mathrm{kT}_{\mathrm{e}}\right)
$$

where I' and I are the emission intensities of a line in the afterglow and steady-state times, respectively, $\gamma=T_{e} / T_{g}, E_{i o n}$ and $E_{p}$ are the energies of the ion ground state and $p^{\text {th }}$ excited atom level, respectively, and k is Boltzmann's constant. If there is reasonable TE in the plasma and the Saha balance prevails a plot of $\ln \left(I^{\prime} / I\right)$ versus $\left(E_{\text {ion }}-E_{p}\right)$, should give a straight line with a positive slope of $[\gamma-1] / \mathrm{kT}_{\mathrm{e}}$ and an intercept of $3 / 2 \ln \gamma$. A plot of this kind is presented in Figure 3.4.a. using the emission intensities of the lines presented in Table 3.1. The dashed line in Figure 3.4.a is an example of how the data falls when the levels are populated by the Saha balance at partial TE (pTE) conditions ${ }^{46}$ (i.e. in an inductively-coupled plasma (ICP)). An attempt to fit a line to the data points determined in this study would give a negative slope with a y-intercept greater than one. As this would put the electron temperature in the afterglow higher than during the voltage-on period (not a probable option), this is an indication that the GD is either not Saha dominated or not at all close to pTE during the voltage-on period. In fact, lines emitted from levels closest to the IP show the largest I'/I ratios, indicating that the Saha 
relation is considerably less involved during the voltage-on period than would be predicted by the proper balance. Again, this is expected if the loss of electrons and ions to the surroundings restricts the proper recombination balance.

If the population of a level is dominated by the Boltzmann balance when the voltage is on, the response to power cessation will be $\mathrm{e}^{47}$

$$
\ln \left(\mathrm{I}^{\prime} / \mathrm{I}\right)=-\left([\gamma-1] / \mathrm{kT}_{\mathrm{e}}\right) \mathrm{E}_{\mathrm{p}}
$$

To test for a Boltzmann-dominated population of levels the natural logarithm of atom emission ratios $I^{\prime} / \mathrm{I}$ are plotted as a function of $\mathrm{E}_{\mathrm{p}}$ in Figure 3.4.b. If the Boltzmann balance is responsible for populating the argon excited levels the points should fall on a line with a negative slope of $-(\gamma-1) / k \mathrm{~T}_{\mathrm{e}}$. It is evident that the points do not fall on a straight line with a negative slope. However, if we treat the lowest levels (13-13.5 eV) separately, and assume that these are in local TE (LTE) and in reasonable Boltzmann balance with the ground state, lines of best fit through these points at each sampling distance reveal electron temperatures in the region of $9,500- \pm 1500 \mathrm{~K}$ above the gas temperature. The sampling height above the cathode (above $2 \mathrm{~mm}$ ) had very little effect on temperature. This crude method for calculating the electron temperature provides electron temperatures considerably higher than previous reports of $2000-6000 \mathrm{~K}^{61-65}$ This indicates the difficulty in assuming that areas of local TE (LTE) exist in the excited levels, as pointed out by other groups. ${ }^{61,63}$ 

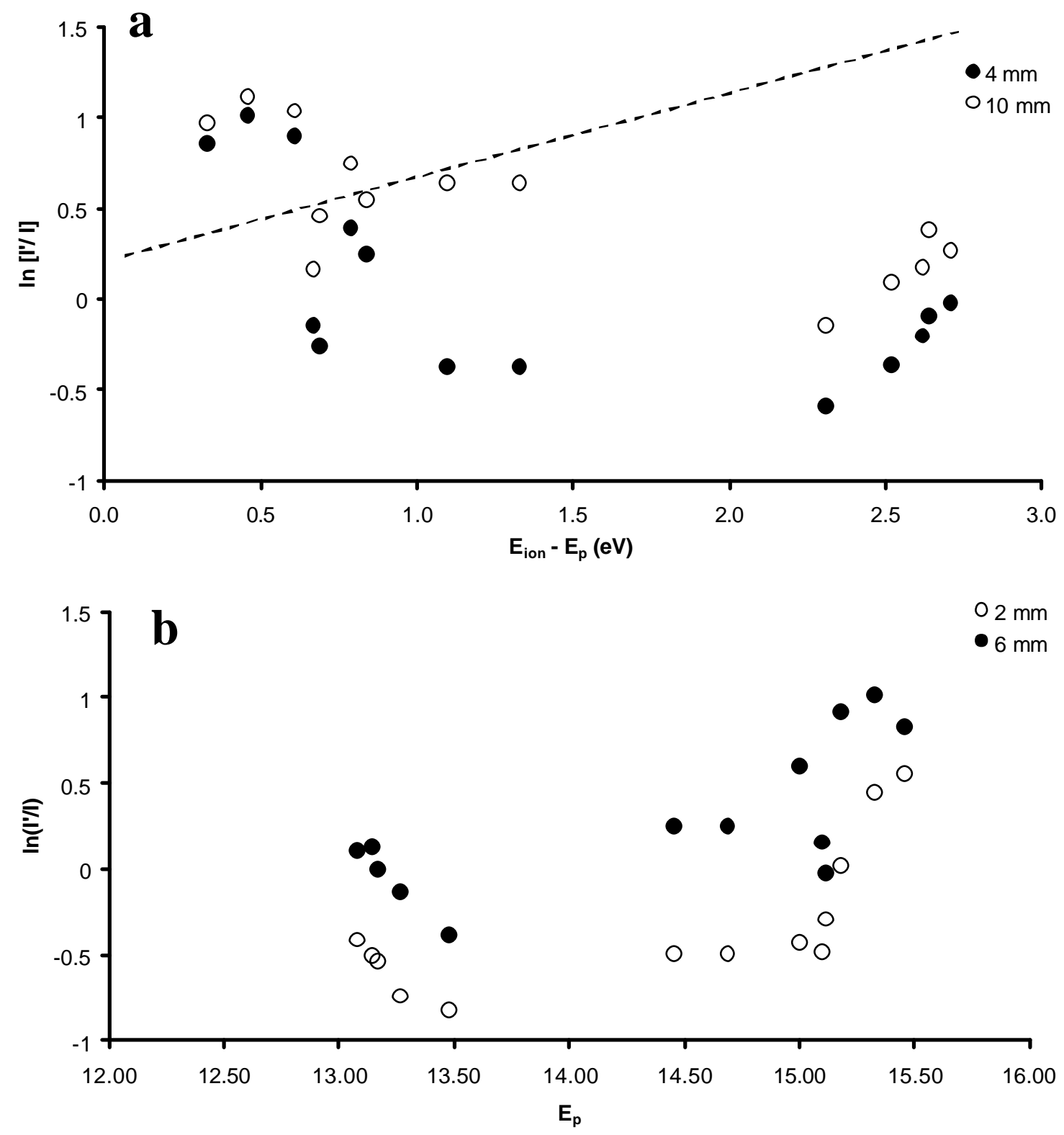

Fig. 3.4. a) Plot to determine the electron temperature from the Saha response of argon atom lines to the voltage termination. b) Plot to determine the electron temperature from the Boltzmann response to voltage termination. The dashed line in a) signifies where the points might fall if Te or pTE existed, i.e. for an ICP plasma. ${ }^{46,47}$ 
Using the same methods described above for argon the emission ratios of the copper atom lines given in Table 3.3 also provided afterpeak/steady-state emission ratios for the determination of $T_{e}$ and the state of TE in the PGD plasma. Figure 3.5.a determines the excited state population inversion observed with that expected in the Saha balance. Akin to argon, the points do not provide a positive slope-as expected for plasmas that are even reasonably close to TE. In fact, lines within $2 \mathrm{eV}$ of the IP deviate more from the Saha balance than do the argon levels in this region. Based on the smaller ionization potential of copper compared to argon, one would expect the copper to be more highly ionized and therefore more likely for the excited states be in the Saha balance. That this is not the case is clear evidence for the extent to which the plasma deviates from TE conditions, and the degree to which electrons and ions are lost to the chamber walls.

Figure 3.5.b is a plot of the copper atom emission ratios used to determine the extent of the Boltzmann balance in the plasma. Again, the points do not suggest a negative slope as predicted by the transition from an equilibrium to a recombining plasma. This can be taken as further evidence that the GD plasma is far from TE conditions when the plasma voltage is terminated at $5 \mathrm{~ms}$. 

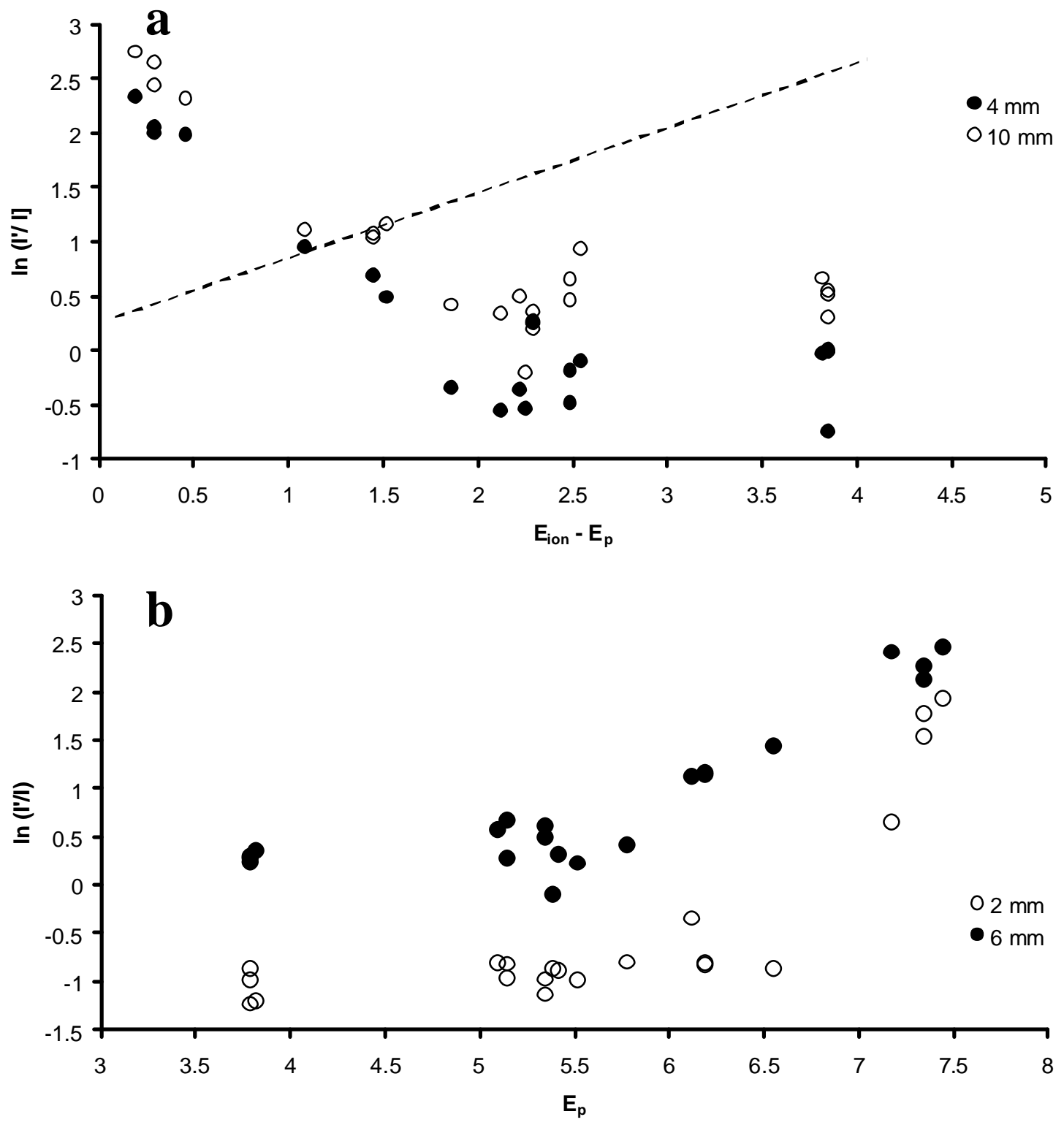

Fig. 3.5. a) Plot to determine the electron temperature from the Saha response of copper atom lines to the voltage termination. b) Plot to determine the electron temperature from the Boltzmann response to voltage termination. The dashed line in a) signifies where the points might fall if Te or pTE existed, i.e. for an ICP plasma. ${ }^{46,47}$ 
Using conditions similar to those presented here, it has been shown that the states below a given level (the so-called Griem level) in ionizing plasmas can be overpopulated by as much as 8 orders of magnitude. ${ }^{56}$ The upward flow of energy from the ground state and the continual loss of ions and electrons to the surroundings cause this overpopulation in the lower levels. Although the Boltzmann balance is a poor descriptor in these circumstances (as we have seen), other balances can be satisfied to determine the distribution of excited states. These balances are electron-density sensitive and are given in Figure 3.6. If the electron density is below $10^{-10} \mathrm{~cm}^{-3}$, excited levels decay to the ground state via radiative transitions rather than by the proper electron de-excitation demanded by the Boltzmann balance. Under these circumstances, the Corona balance determines that the distribution of energy states is populated proportionally to $\mathrm{p}^{-0.5} \cdot 34,35$

At high electron densities $\left(>10^{15} \mathrm{~cm}^{-3}\right)$ the plasma enters the saturation phase. In this case, ladder-like excitation by electrons continues up the higher levels until the population of energy levels is proportional to $\mathrm{p}^{-6} \cdot 33-35$ For electron densities between these two extremes, i.e. the $\sim 10^{11}-10^{12} \mathrm{~cm}^{3}$ densities found in typical glow discharges, ${ }^{61,62}$ the population of energy levels will lie between the extremes of $\mathrm{p}^{-0.5}$ and $\mathrm{p}^{-6}$ dependence. Regardless of the electron density, ${ }^{66}$ an ionizing plasma will always have an overpopulation of lower levels and an under-population of excited levels with respect to an equilibrium plasma defined by the Boltzmann balance. 


\section{Ionizing Plasma}

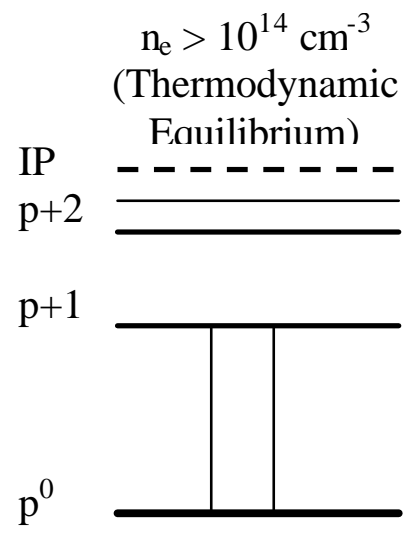

Boltzmann Balance

$\mathrm{n}(\mathrm{p}) / \mathrm{g}(\mathrm{p}) \propto \mathrm{e}^{-\mathrm{E} / \mathrm{kT}}$ $\mathrm{n}_{\mathrm{e}}<10^{10} \mathrm{~cm}^{-3}$

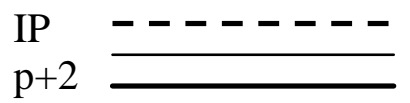

$\mathrm{p}+1$

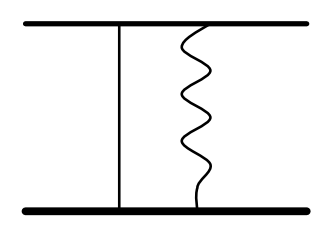

Corona Balance $\mathrm{n}(\mathrm{p}) / \mathrm{g}(\mathrm{p}) \propto \mathrm{p}^{-0.5}$ $\mathrm{n}_{\mathrm{e}}>10^{14} \mathrm{~cm}^{-3}$

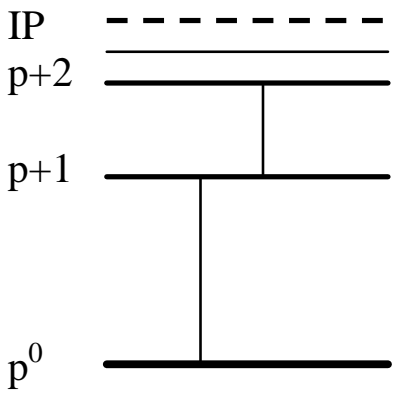

Saturation Balance $\mathrm{n}(\mathrm{p}) / \mathrm{g}(\mathrm{p}) \propto \mathrm{p}^{-6}$

Fig. 3.6. Examples of electron excitation mechanisms at different electron number densities in an ionizing plasma. Solid lines and wavy lines represent electron and photon-accompanied transitions, respectively. $n(p) / g(p)$ is the population density per unit statistical weight, $\mathrm{p}^{0}$ is the ground state and IP is the ionization potential. Between $10^{10}$ and $10^{14} \mathrm{e}^{-} \mathrm{cm}^{-3}$ the Griem and Byron criterion determine which levels of $\mathrm{p}$ are governed by which balance, ${ }^{33,36}$ Above $10^{14} \mathrm{e}^{-} \mathrm{cm}^{-3}$ the Byron criterion divides the lower levels $(\mathrm{p}<7)$ and the upper levels $(\mathrm{p}>10)$ into the Boltzmann balance and saturation excitation balance, respectively. ${ }^{33,36}$ 


\subsubsection{Departure from the Planck balance}

As well as the deviation from TE caused by the different forces applied to the different particles in the discharge, and to the losses of electrons and ions, there are other factors that add to the departure of GDs from TE. Radiation is lost to the surroundings (hence the observation of the glow) so the re-absorption of radiation by lower-excited states will decrease. This deviation from the Planck balance also will tend to over-populate the lower energy levels.

\subsubsection{Additional factors contributing to TE departure}

The velocity distribution of electrons is known to be non-Maxwellian in steady-state GD sources, ${ }^{1,62}$ and this contributes strongly to deviations from TE. The non-Maxwellian distribution is caused, in part, by ionization reactions that release electrons with high kinetic energies. ${ }^{62}$ For example, Penning ionization (PI)

$$
\mathrm{Ar}^{\mathrm{m}}+\mathrm{M} \rightarrow \mathrm{Ar}^{0}+\mathrm{M}^{+}+\mathrm{e}^{-}(\mathrm{x} \mathrm{eV})
$$

releases electrons with kinetic energies dependant on the IP of $M$. If $M$ is another metastable argon atom (IP $\sim 4.2 \mathrm{eV}$ ) the kinetic energy of the expelled electrons will have $\sim 7.3 \mathrm{eV}$ of kinetic energy. These electrons contribute to the number of high-energy electrons in the electron-energy-distribution-function (EEDF) and distort the Maxwellian distribution. Penning ionization of the sputtered atoms also contributes strongly to the number of high-energy electrons in the plasma. This is demonstrated by occurrence of higher electron temperature measurements in plasmas having cathode materials with lower ionization potentials. ${ }^{62}$ Associative ionization, also known as the Hornbeck-Molnar reaction, ${ }^{67}$ 


$$
\mathrm{Ar}^{*}+\mathrm{M} \rightarrow \mathrm{ArM}^{+}+\mathrm{e}^{-}(\mathrm{x} \mathrm{eV})
$$

is another mechanism by which the influx of electrons can disrupt the Maxwellian distribution of velocities. If $\mathrm{M}$ is a ground state argon atom, the onset for this reaction occurs at $\sim 15 \mathrm{eV}$, implying that the metastable states are not involved in this reaction. For metal-noble gas molecular ions, ${ }^{68}$ however, the metastable states of argon are energetically viable reactants for this mechanism of ionization.

\subsubsection{Transition from an ionizing- to a recombining plasma}

The above discussion has shown that the distribution of excited states during the voltage-on period is heavily over populated in the lower levels with respect to the Boltzmann balance, and under populated in the upper levels with respect to the Saha balance. These two observations are indications that the GD is dominantly ionizing while the voltage is applied. ${ }^{33-35}$ If we assume that the electron temperature decays to the bath gas temperature when the voltage is removed, ${ }^{33,50}$ and that three-body (and possibly twobody) recombination is then possible, the plasma will transition to a recombining one. In the recombining plasma, a downward flow of energy overpopulates the higher levels with respect to the Saha-Boltzmann balance. Figure 3.7 shows examples of the populating mechanisms that are dominant at different electron densities in the recombining phase of plasmas. 


\section{$\underline{\text { Recombining Plasma }}$}

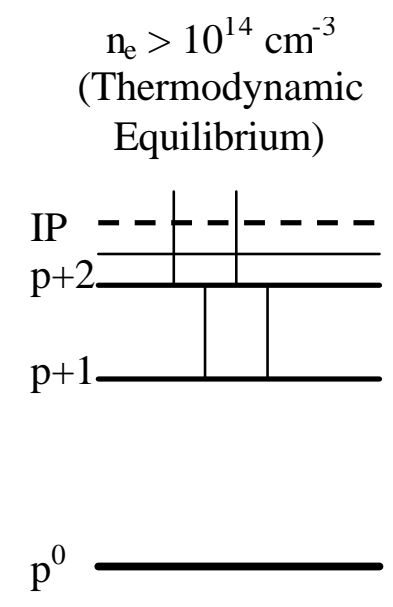

Boltzmann Balance $\mathrm{n}(\mathrm{p}) / \mathrm{g}(\mathrm{p}) \propto \mathrm{e}^{-\mathrm{E} / \mathrm{kT}}$

$$
\mathrm{n}_{\mathrm{e}}<10^{10} \mathrm{~cm}^{-3}
$$

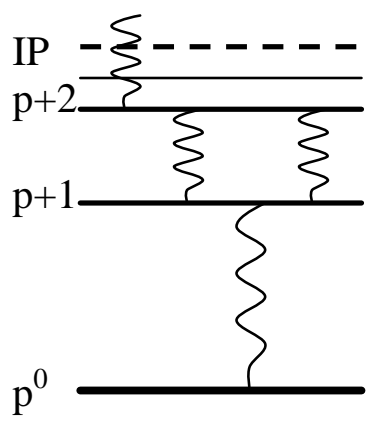

Capture-RadiativeCascade Balance $\mathrm{n}(\mathrm{p}) / \mathrm{g}(\mathrm{p}) \propto \mathrm{p}^{1.5}$

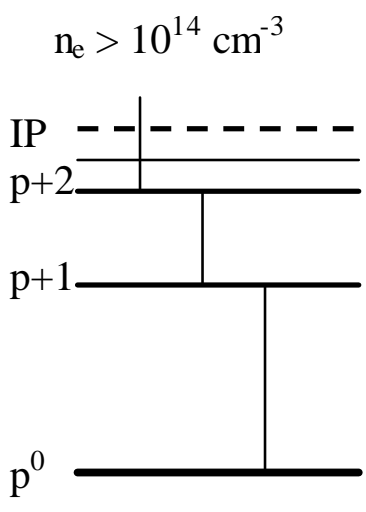

SaturationDeexcitation Balance $\mathrm{n}(\mathrm{p}) / \mathrm{g}(\mathrm{p}) \propto \mathrm{p}^{-6}$

Fig. 3.7. Examples of electron de-excitation mechanisms at different electron number densities in a recombining plasma. Solid lines and wavy lines represent electron and photon accompanied transitions, respectively. $\mathrm{n}(\mathrm{p}) / \mathrm{g}(\mathrm{p})$ is the population density per unit statistical weight, $\mathrm{p}^{0}$ is the ground state and IP is the ionization potential. Above $10^{14} \mathrm{e}^{-} \mathrm{cm}^{-3}$ the Byron criterion divides the lower levels $(\mathrm{p}<7)$ and the upper levels $(\mathrm{p}>10)$ into the Boltzmann balance and saturation deexcitation balance, respectively. ${ }^{33,36}$

At low electron densities, the capture-radiative-cascade (CRC) balance dominates and the density of (neutral) states is proportional to $\mathrm{p}^{1.5} \cdot 33,34,36,37$ In this phase, depopulation is purely radiative. If the electron density exceeds the Griem boundary, the depopulation of levels becomes collision-controlled. In this domain, the Saha-Boltzmann 
balance describes the higher levels and the saturation de-excitation balance describes the lower levels. Lower levels occupied by the saturation de-excitation balance are then populated proportionally to $\mathrm{p}^{-6}$. The boundary that distinguishes the quantum levels populated is called the Byron boundary and is discussed in detail elsewhere. ${ }^{33,36}$ Electron densities in the PGD used here are expected to fall in the CRC zone, and therefore provide a population inversion in the afterglow related to $n(p) / g(p) \sim p^{1.5}$.

The afterglow/steady state emission ratios measured for the various excited levels of atoms demonstrate the diversity in the population of energy states between the ionizing and recombining phases. When the electron density in the PGD is below $10^{13} \mathrm{~cm}^{-3}$, the population of states in the ionizing and recombining phases will be proportional to $\mathrm{p}^{-0.5}$ and $\mathrm{p}^{1.5}$, respectively. The ratio of the afterglow/steady-state emission signals would then be expected to scale according to $\mathrm{p}^{1}$, i.e. scale linearly with $\mathrm{p}$. Figures 3.4.b and 3.5.b suggest that the relationship between the afterglow/steady-state emission ratio and the energy levels (an approximation for the quantum levels) is in fact linear above a certain value ( $15 \mathrm{eV}$ for $\mathrm{Ar} \mathrm{I}, \sim 5.5 \mathrm{eV}$ for $\mathrm{Cu}$ I). This description provides convincing evidence that recombination reactions between atomic ions and electrons in the CRC domain account for the majority of emissions occurring in the afterglow.

\subsubsection{Phase transitions at the pulse-onset}

As well as the afterglow, the onset of the pulsed voltage is a time of significant change in the plasma characteristics. Between the initial gas breakdown and the steadystate condition, changes in populations are energy-level-dependant. For example, the emission from the $4 \mathrm{p}$ states (see Figure 3.2.d) demonstrates a significant prepeak, 
whereas the emission lines for higher levels (i.e. $7 \mathrm{~s}$ and $4 \mathrm{~d}$ states in Figure 3.2.b and 3.2.c, respectively) show opposite behavior. Their emission intensities are smaller at $2 \mathrm{~ms}$ than at $5 \mathrm{~ms}$ (at distances $>4 \mathrm{~mm}$ ). The copper atom lines demonstrate similar behavior: lower-energy lines show a pronounced pre-peak but emissions from higher energy lines are weaker in the prepeak domain than in the steady-state. Lines between the upper and lower levels, i.e. the $5 \mathrm{p}$ states, generally reach a steady-state signal quite early in the pulse and do not fluctuate considerably until voltage termination. The initial gas breakdown therefore appears to selectively overpopulate the lower levels and under populate the upper levels with the respect to the steady state signals.

Figure 3.8 shows the afterpeak/steady-state and afterpeak/prepeak emission ratios for all the argon lines in Table 3.1 plotted as a function of the energy of the emitting levels. At $6 \mathrm{~mm}$ and $10 \mathrm{~mm}$ above the cathode, the afterpeak/pre-peak ratios are significantly larger than the afterpeak/steady-state ratios for the higher lying states. This implies that the higher lying states are considerably less populated in the pre-peak than during the steady-state. The reason that the lower levels are more highly populated in the pre-peak (as seen for the 4p states Figure 3.2.d) stems from the initial conditions of the plasma. At the onset of the voltage, the argon ground state is severely overpopulated and the applied electric field decreases linearly between the cathode and anode. As fast electrons ionize the argon, the charged-particle number density increases until spacecharge factors prevent any further increase. ${ }^{25}$ Initially, the lowest excited states populate via electron excitation from the ground state according to the corona balance, but as the electron number density increases these lower-excited states become over-populated and subsequently excite to increasingly higher levels according to the saturation balance. The 
lower excited levels therefore go through a maximum as the populating/depopulating mechanisms shift from the corona to the saturation balance. Because the plasma is more over-populated in the lower levels during the pre-peak than during the steady-state, the negative glow is more ionizing in the pre-peak than the steady-state. This shift in plasma properties from a very-ionizing plasma in the pre-peak to a lesser-ionizing plasma in the steady-state also explains the under-population of upper levels in the pre-peak with respect to the steady-state. 

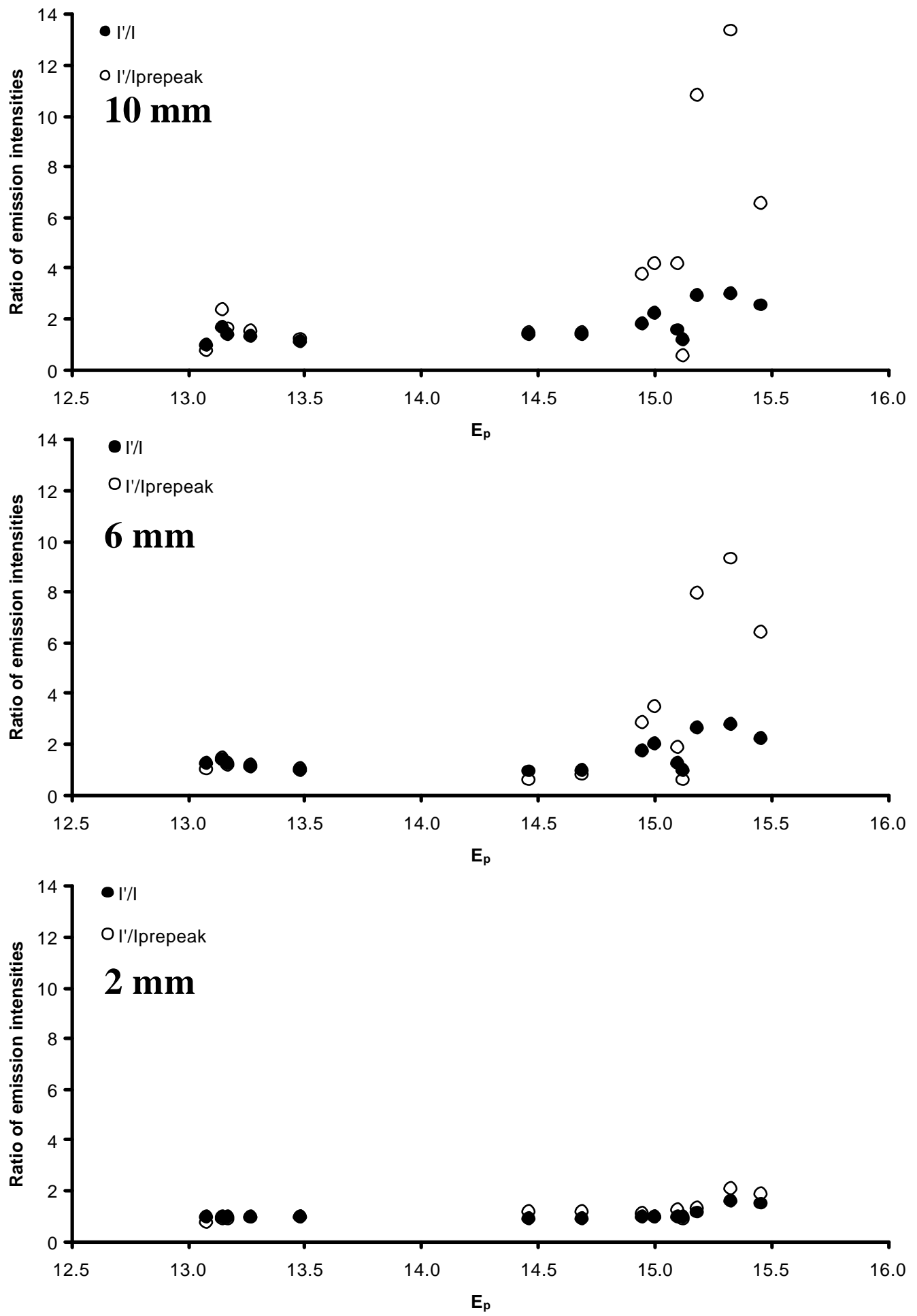

Fig. 3.8. Plots of the afterpeak/steady-state (I'/I) and afterpeak/prepeak (I'/I $\left./ I_{\text {prepeak }}\right)$ as a function of the energy level of the emitting level at different sampling distances above the cathode. 
As Figure 3.8.c demonstrates, while the space-charge factors greatly affect the distribution of states above the cathode darkspace, little effect is observed within the cathode darkspace. At $2 \mathrm{~mm}$ the afterpeak/steady-state and afterpeak/pre-peak ratios are very similar for all the atom lines studied. This implies that the population of states does not change between the pre-peak and steady-state values. In the steady-state plasma, the applied field no longer drops linearly between the two electrodes, but drops almost entirely in the cathode fall (the distance between the cathode surface and the negative glow-usually $\sim 0.5-2 \mathrm{~mm}) .{ }^{69}$ In addition to the reduced electron excitation effect in the cathode darkspace, excitation contributions from fast atom and fast ion excitation reduce the involvement of electron excitation in the population of states in this region. This implies that any vacillation in the electron excitation contribution to the population of states is less noticeable in the cathode darkspace than in the negative glow. This explains why, in Figure 3.8.c, the transformation between the pre-peak and steady-state distribution of states is small and largely unaffected by the development of the negative glow.

The populations of states, and the departure from TE conditions, appear to be somewhat dependable on the plasma lifetime, or time-gated detection. Operating the PGD at shorter pulse widths, or collecting time-gated emission signals at shorter times, places the ionizing plasma further from TE conditions and preferentially populates the lower lying levels while effectively attenuating emissions from the higher excited levels. Operating at longer pulse widths $(>4 \mathrm{~ms})$, or time-gated monitoring at longer times, encourages the formation of, and therefore emission from, the higher-level lines. 
In agreement with the previous discussion, Yan et al. recently demonstrated that increasing the pulse width from $2-200 \mu$ s significantly affected the relative populations of various levels in the copper and argon atom and ion states. ${ }^{70}$ The low-lying resonance state of copper was found to be the most enhanced in the pre-peak relative to the steadystate domain, in agreement with the current findings. Unfortunately, Yan et al. only examined one line for each atom and ion in the plasma so the distribution of excited states could not be confirmed as in agreement with those presented here.

\subsubsection{Argon ion emissions}

Mass spectrometric studies ${ }^{16,40,59,71}$ show that argon ion densities are almost negligible 100 microseconds after pulse termination. It is not surprising, therefore, that their emissions do not prevail in the afterglow. ${ }^{32,40}$ In this study it is also found that emission from excited argon ions do not exhibit an afterpeak and that the intensity follows an approximate exponential decay after the plasma is extinguished. Figure 3.9 shows the temporal profiles of the $476.4 \mathrm{~nm}$ line $\left(4 \mathrm{p}^{2} \mathrm{P}^{0}-4 \mathrm{~s}{ }^{2} \mathrm{P}, 19.87-17.26 \mathrm{eV}\right)$ as a function of distance from the cathode. Steady-state intensities are reached well before 5.0 ms at all distances and all decay rapidly after pulse termination. Because the tailing is elongated by the $1 \mathrm{M} \Omega$ coupling between the PMT and the digital oscilloscope, a decay constant is not calculated. 


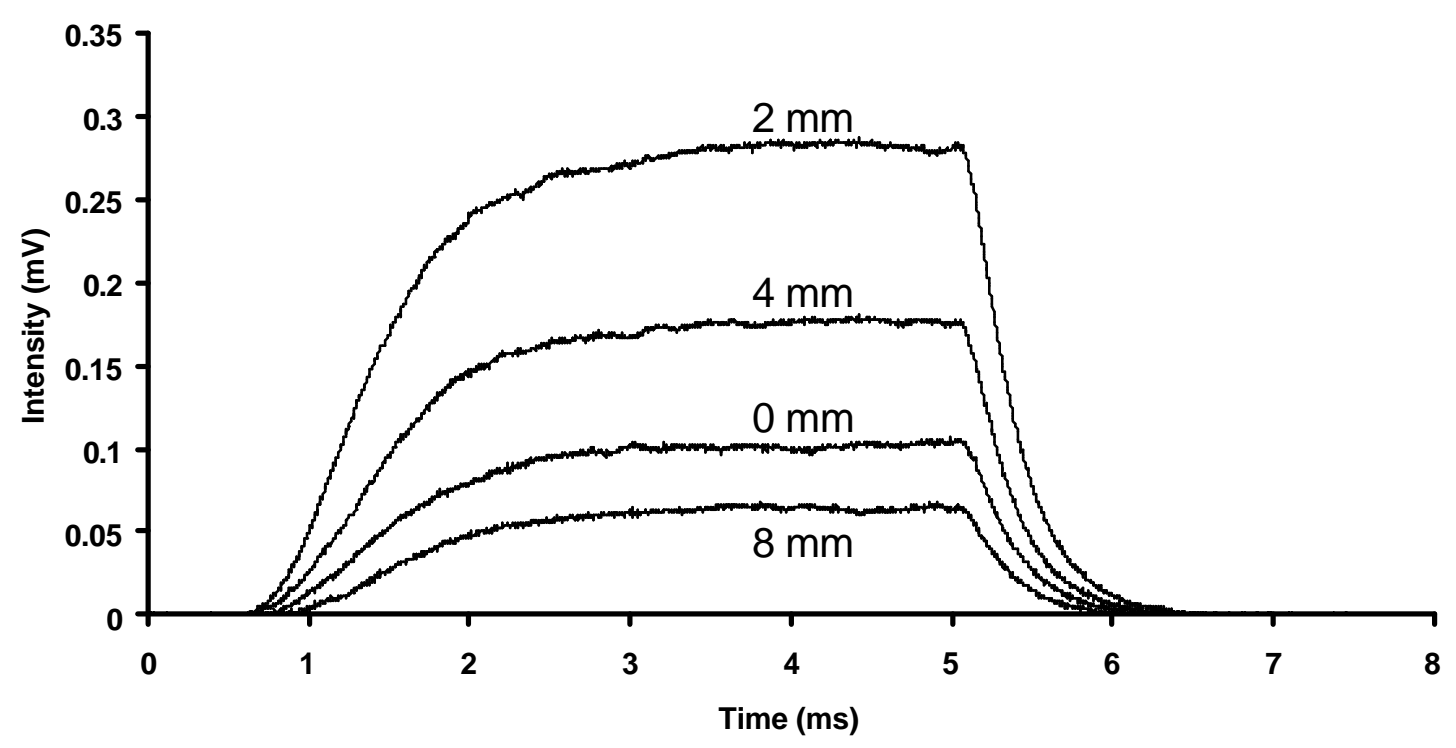

Fig. 3.9. Plot of the emission intensity of the argon ion line at $476.4 \mathrm{~nm}$ as a function of time to show the steady-state condition reached and the absence of an afterpeak. Pulse length $5.0 \mathrm{~ms}, 25 \%$ duty cycle, 0.8 Torr Ar, $\sim 1.5 \mathrm{~W}$ peak power.

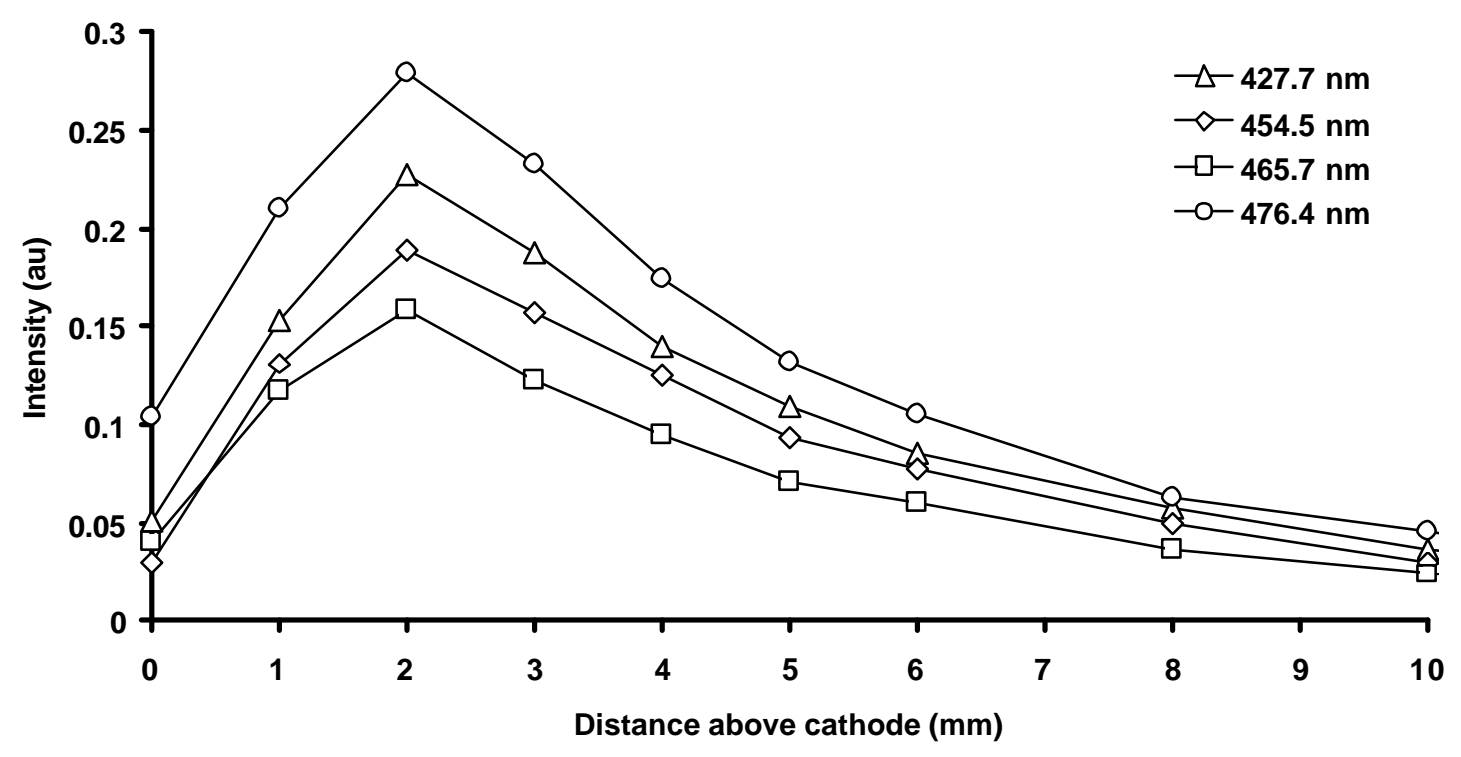

Fig. 3.10. Plot of argon ion emissions for several lines as a function of distance to show the spatial distribution of these levels. Pulse length $5.0 \mathrm{~ms}, 25 \%$ duty cycle, 0.8 Torr Ar, $1.5 \mathrm{~W}$ peak power. 
All the argon ion transitions observed (refer to Table 3.2) followed almost identical behavior and are presented, in part, in Figure 3.10. A maximum emission intensity is reached at $2.0 \mathrm{~mm}$ above the cathode, while a minimum is obtained at the surface. This suggests that fast atom/ion bombardment does not play a significant role in populating Ar ion states. The emission intensity decreases at distances beyond $2.0 \mathrm{~mm}$ from the surface. This behavior is indicative of the electron excitation that dominates the area between $2.0-5.0 \mathrm{~mm}$. The similarity in behavior between all the argon ion lines is rationalized as follows: the upper energy levels of each transition lie within a $3 \mathrm{eV}$ spread and this spread lies $\sim 36 \mathrm{eV}$ above the argon atom ground state. Electron excitation is the only feasible mechanism of excitation and this will not be selective over the energy range considered.

Figure 3.11 presents two-dimensional spatial maps for various times during the pulse cycle for the $476.4 \mathrm{~nm}$ line. The maps have been normalized to the most intense point in the series and each contour line represents a $10 \%$ change in emission intensity. Upon power cessation at $5.0 \mathrm{~ms}$, there is a small delay, $\sim 0.1 \mathrm{~ms}$, after which the emission intensity undergoes a rapid decrease. In contrast to argon atom emissions, the argon ion emissions do not alter as a function of distance from the cathode. This simple decay of radiation demonstrates the inability of the recombining plasma to populate these high energy levels in the absence of applied power.

The optical apparatus employed cannot directly observe the ground state argon ions in the discharge. However, the ${ }^{3} \mathrm{P}_{3 / 2}$ ground state argon ion, $0.18 \mathrm{eV}$ above the ${ }^{3} \mathrm{P}_{1 / 2}$ ground state ion, overlaps efficiently with an excited sate of the copper ion. ${ }^{72-74}$ 
Following a charge transfer reaction between the ${ }^{3} \mathrm{P}_{3 / 2}$ state ion and the copper ground state atom,

$$
\mathrm{Ar}^{+}\left({ }^{3} \mathrm{P}_{3 / 2}\right)+\mathrm{Cu}^{0} \rightarrow \mathrm{Cu}^{+*}+\mathrm{Ar}^{0} \rightarrow \mathrm{Cu}^{+}+\mathrm{Ar}^{0}+h v(224.7 \mathrm{~nm})
$$

the excited copper ion product can radiatively decay to a lower excited state, one of which transitions occurs at $224.7 \mathrm{~nm}$. Therefore, indirect monitoring of the ${ }^{3} \mathrm{P}_{3 / 2}$ ground state argon ion population is possible by monitoring the emission line at $224.7 \mathrm{~nm}$. As presented elsewhere, ${ }^{16,17}$ the emission from the copper ion at $224.7 \mathrm{~nm}$ maximizes further from the cathode surface than the emission of the argon ion lines discussed above. Also, the $224.7 \mathrm{~nm}$ line does not reach a steady-state condition in the $5 \mathrm{~ms}$ pulse, as do the other argon ion and copper ion lines studied. This suggests that 1) many more argon ions form in the ground state in the negative glow area than are optically detected, and 2) the argon ion ground state population continues to increase over the $5 \mathrm{~ms}$ period. Mass spectrometric measurements (discussed in Chapter 4) of Ar ion populations support this finding. 


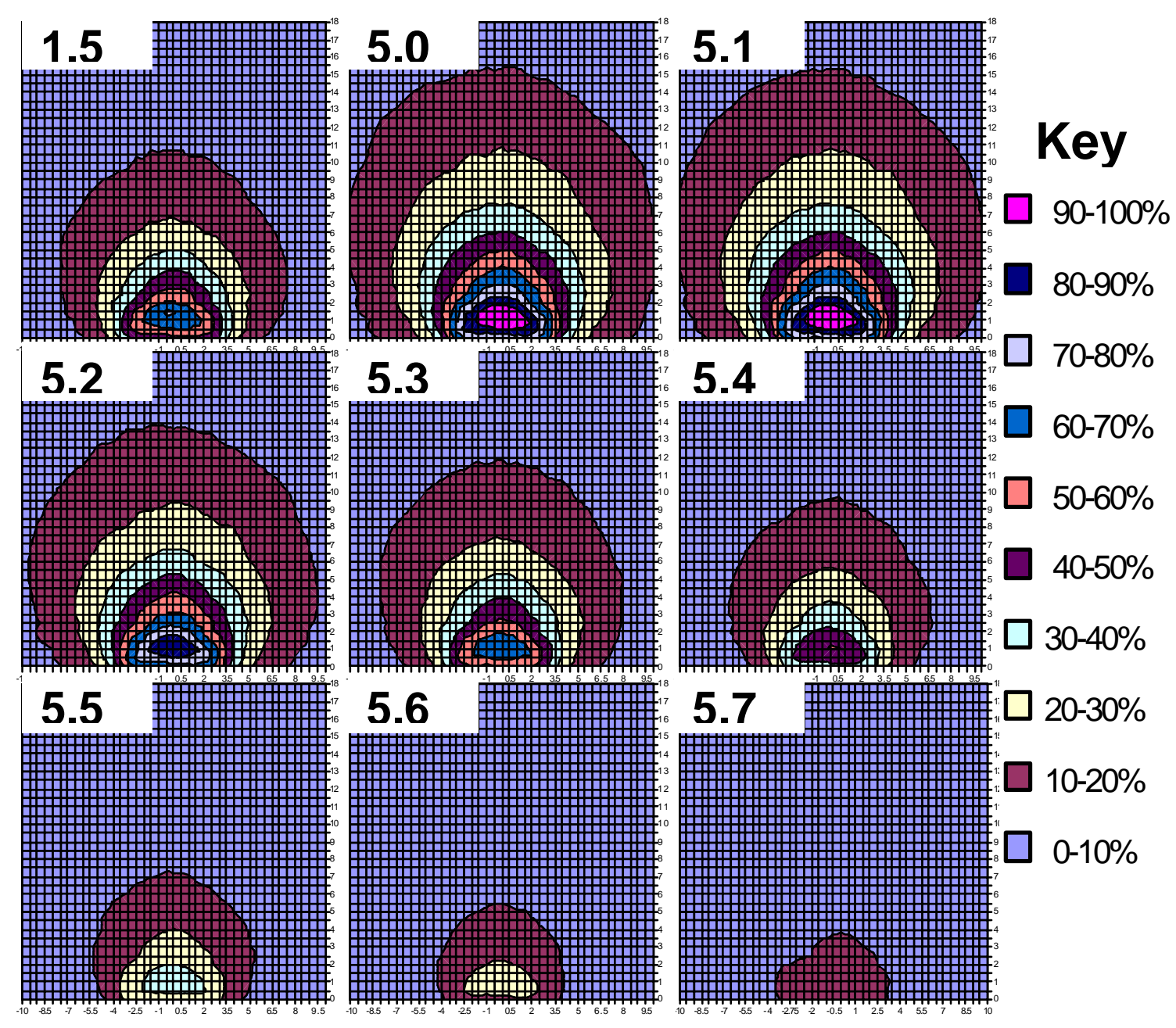

Fig. 3.11. Two-dimensional maps of the normalized argon ion emission at $476.4 \mathrm{~nm}$ to show the evolution of the excited levels over the pulse cycle (times shown on each map are in ms) Pulse length $5.0 \mathrm{~ms}, 25 \%$ duty cycle, 0.8 Torr Ar, $\sim 1.5 \mathrm{~W}$ peak power. 


\subsubsection{The mechanism of electron-ion recombination}

Bogearts and Gijbels recently developed a collisional-radiative model for the microsecond-PGD ${ }^{30}$ and noted difficulties in relating measured and predicted recombination rate constants. Until now, the terms 'recombination' and 'recombining plasma' have been used rather loosely and without regard for the specific reactions and rates to validate the arguments. In the following discussion, we attempt to resolve this issue.

To adhere to the law of conservation of momentum and conservation of energy, the capture of an electron by an atomic ion must be accompanied by the release of energy in/by a third body. ${ }^{1}$ Until now, it has been assumed that the third body is an electron, as defined by the Saha relation in Equation 3.5 (repeated in reverse here for convenience).

$$
\mathrm{Ar}^{+}+\mathrm{e}_{\text {slow }}^{-} \mathrm{e}_{\text {slow }}^{-} \leftrightarrow \operatorname{Ar}^{\mathrm{p}}+\mathrm{e}_{\text {fast }}^{-}
$$

The rate constant for this reaction at $T_{e}=1000 \mathrm{~K}$ and $n_{e}=10^{11} \mathrm{~cm}^{3}$ is approximately $10^{-11}$ $\mathrm{cm}^{-3} \mathrm{~s}^{-1} \cdot{ }^{36}$ Assuming the electrons cool to the bath gas temperature and electrons are not immediately lost by any other mechanism, these values will reasonably represent the values present in the early afterglow. These values give a three-body recombination rate of $\sim 10 \mathrm{sec}^{-1}$, or a lifetime for the ions of $\sim 0.1 \mathrm{sec}$. This rate is at least three orders of magnitude too small to account for the appearance of the afterglow at $\sim 150 \mu$ s. Threebody recombinations involving a second argon atom to dissipate the excess energy have been estimated to be in the region of $10^{-11} \mathrm{x} \mathrm{P} \mathrm{cm}^{3} \mathrm{sec}^{-1}$, where $\mathrm{P}$ is the gas pressure in Torr (Equation taken from ${ }^{22}$; Original source is ${ }^{75}$ ). At an operating pressure of 0.8 Torr (used here), with $\mathrm{n}_{\mathrm{e}} \approx \mathrm{n}_{\mathrm{Ar}} \approx 10^{11} \mathrm{~cm}^{-3}$, this corresponds to a recombination rate of $\sim 10^{11}$ $\mathrm{cm}^{-3} \mathrm{sec}^{-1}$, or $\sim 1 \mathrm{sec}^{-1}$. This gives a lifetime for the ions of $\sim 1 \mathrm{~s}$, which is also too slow to 
account for the afterglow emissions. An alternative recombination mechanism is twobody recombination, or radiative recombination.

$$
\mathrm{Ar}^{+}+\mathrm{e}_{\text {slow }}^{-} \leftrightarrow \operatorname{Ar}^{\mathrm{p}}+h v
$$

In this case, the third 'body' is a photon. This process can be thought of as the reverse of photo-ionization and has a rate coefficient of $\sim 10^{11} \mathrm{~cm}^{3} \mathrm{sec}^{-1}$. Again, at electron densities of $\sim 10^{11} \mathrm{~cm}^{-3}$, the radiative recombination rate should be on the order of $1 \mathrm{sec}^{-1}$. This is also too slow to account for the afterglow at $\sim 150 \mu$ s (requiring a rate $\left.>5000 \mathrm{sec}^{-1}\right)$.

An additional recombination method is dissociative recombination, in which an argon-dimer ion captures a slow electron to form a neutral dimer in a repulsive state that quickly dissociates.

$$
\mathrm{Ar}_{2}^{+}+\mathrm{e}_{\text {slow }}^{-} \rightarrow \mathrm{Ar}_{2}^{*} \rightarrow \mathrm{Ar}^{\mathrm{p}}+\mathrm{Ar}_{\text {fast }}
$$

This can be thought of as three-body recombination in which the third body is conveniently attached to the argon ion. Numerous studies have shown that this reaction is considerably more efficient than two-body recombination (Equation 3.12), having a rate coefficient of $\sim 10^{-7} \mathrm{~cm}^{3} \mathrm{sec}^{-1} \cdot 50,76,77$ At an electron density of $\mathrm{n}_{\mathrm{e}} \varphi 10^{11} \mathrm{~cm}^{-3}$, the recombination rate is $\sim 1000 \mathrm{sec}^{-1}$. This gives a recombination rate in excellent agreement with the observed decay rates for the emission from excited states of Argon. However, an additional step is required to form the argon dimers from the argon ions

$$
\mathrm{Ar}^{+}+2 \mathrm{Ar} \leftrightarrow \mathrm{Ar}_{2}^{+}+\mathrm{Ar}_{\text {fast }}
$$

and this reaction has a rate coefficient of $\sim 3 \times 10^{-31} \mathrm{~cm}^{6} \mathrm{sec}^{-1}$ at $300 \mathrm{~K} .^{78-80}$ The formation rate for argon dimer ions at $n_{\mathrm{Ar}} \varphi 2.8 \times 10^{16} \mathrm{~cm}^{-3}$ (used here) is then expected to be $\sim 230$ $\mathrm{sec}^{-1}$. Biondi estimates this reaction to occur at $\sim 10^{4} \mathrm{sec}^{-1}$ at $10 \mathrm{Torr},{ }^{50}$ but does not give 
any indication of the pressure dependency. Assuming the rate scales proportionally to $\mathrm{n}_{\mathrm{Ar}}{ }^{2}$, the formation rate at 0.8 Torr should be on the order of $\sim 10^{2} \mathrm{sec}^{-1}$, in agreement with the above calculation of $\sim 230 \mathrm{sec}^{-1}$. If argon ions recombine via this two-step mechanism, the formation rate for the argon dimer ion will be the rate-limiting step in the process and presumably would not be detectable in mass spectrometric analyses. The lifetime of the argon ions should be on the order of $4 \mathrm{~ms}$-within two orders of magnitude of the actual loss rate.

This process seems the most probable mechanism considered so far. However, studies have shown that based on the dissociation energy and IP of the neutral dimer, the excited states of $\mathrm{Ar}^{\mathrm{p}}$ that are formed in the dissociation of the neutral argon dimer can only occupy levels $\leq 14.47 \mathrm{eV} .^{81}$ This implies that emissions can only come from the $3 \mathrm{~d}$ ', $4 p$ and $4 p$ ' states. Emissions from these levels should be dominant in the afterglow if dissociative recombination is indeed the overriding recombination mechanism. The decay rates between 5.5-7 $\mathrm{ms}$, at $4 \mathrm{~mm}$ above the cathode were measured for some of the lines in Table 3.1 and are given in Figure 3.12. The decay rates for lines $\leq 14.47 \mathrm{eV}$ are not slower than the decay rates for other lines. Counter-intuitively, it appears that these lower levels have a slightly faster decay rate than the other levels.

Okada and Sugawara developed a mathematical model for the afterglow of a pulsed plasma to account for the prolonged emissions and differences in population states for different energy levels of the argon atoms. However, the model assumes that only dissociative recombination occurs, and consequently they only investigated emissions originating from the $5 \mathrm{p}$ and $4 \mathrm{p}$ states. The authors made no comment regarding emissions from states above those that can be populated by dissociative recombination (i.e. above 
$14.47 \mathrm{eV}){ }^{77}$ Okada and Sugawara ${ }^{82}$ did show that the $5 \mathrm{p}$ emissions had considerable larger afterpeak/steady-state emission ratios than the $4 p$ states, in agreement with our findings, but their model would predict any of the afterglow emissions we observe above the $5 \mathrm{p}$ state. Finally, their model, and experiments, were based on an operating pressure of 5 Torr argon, which would put the argon dimer formation (equation 3.14) approximately 40 times faster than expected here.

Shiu and Biondi have shown that at elevated temperatures $(7200 \mathrm{~K})$ it is possible that vibrationally excited argon dimers can recombine with electrons to yield excited argon neutral dimers. These have a short lifetime and dissociate to give a ground state and excited state argon atom; the latter having a maximum internal energy of $0.6-0.8 \mathrm{eV}$ above the argon dimer ground state. This implies that the argon neutral formed can potentially occupy levels up to $\sim 15 \mathrm{eV}$; the highest observed atom states resulting from recombination were the $5 \mathrm{~d}$ states. 


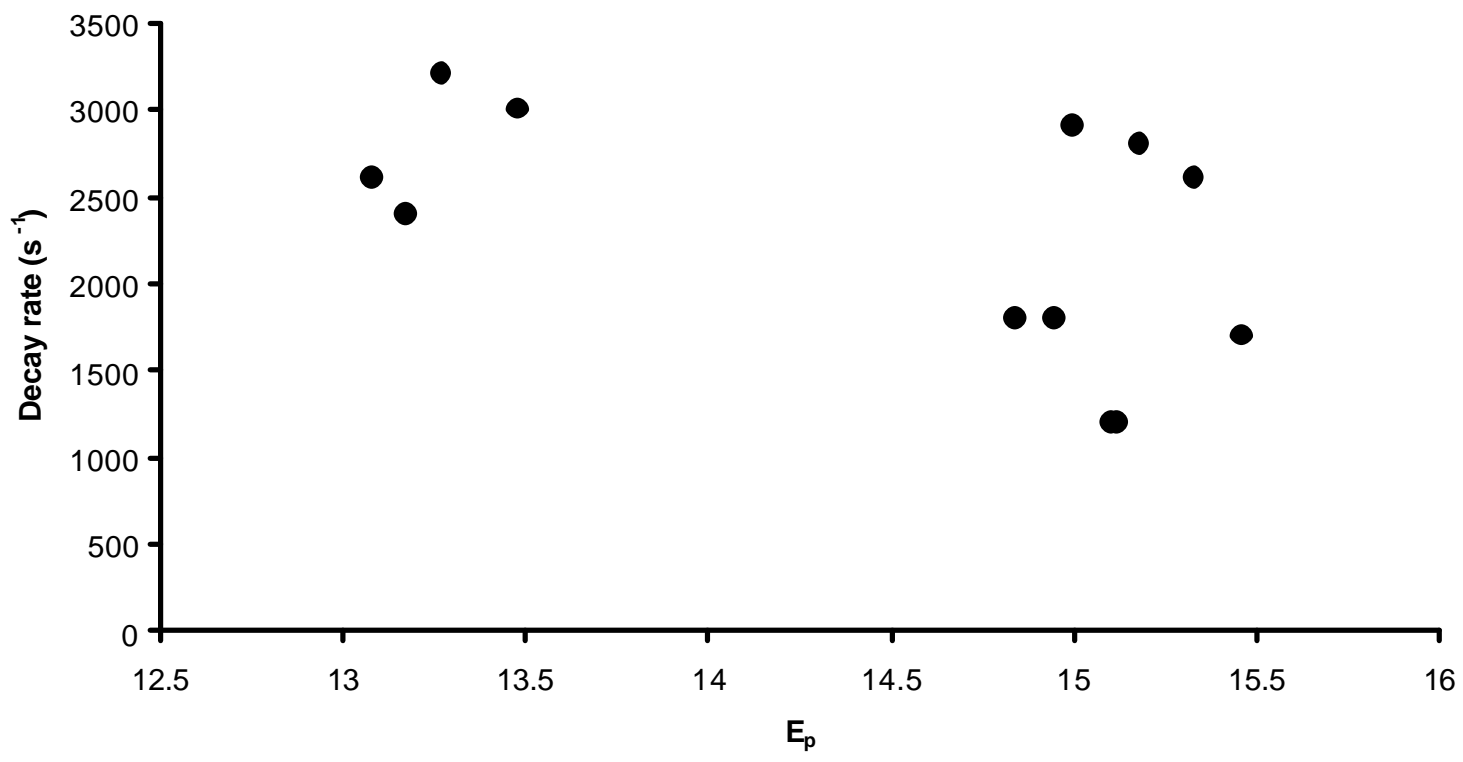

Fig. 3.12. Argon atom decay rates as a function of the upper energy level of the emitting species. Rates were determined from the plots of $\ln (\mathrm{I})$ versus time between 5.4 and $7 \mathrm{~ms}$ and had minimum $\mathrm{R}^{2}$ co-efficients of 0.99 indicating a first order decay. 
In an attempt to find an alternative recombination mechanism to account for the fast recombination rate observed, the effects of gaseous impurities are also considered. One dominant impurity is $\mathrm{H}_{2} \mathrm{O}$, which, given the gas purity and possibility for desorption from chamber walls, could be present at levels of $\sim 10 \mathrm{ppm}$. A sequence of reactions given in Equations 3.15 and 3.16 could then be a feasible method for recombination of argon ions with electrons.

$$
\begin{aligned}
& \mathrm{Ar}^{+}+\mathrm{H}_{2} \mathrm{O} \rightarrow \mathrm{ArH}^{+}+\mathrm{OH} \\
& \mathrm{ArH}^{+}+\mathrm{e}_{\text {slow }}^{-} \rightarrow \mathrm{ArH}^{*} \rightarrow \mathrm{Ar}^{0}+\mathrm{H}_{\text {fast }}
\end{aligned}
$$

Alternatively, the reaction with water could also yield the product

$$
\mathrm{Ar}^{+}+\mathrm{H}_{2} \mathrm{O} \rightarrow \mathrm{Ar}^{0}+\mathrm{H}_{2} \mathrm{O}^{+}
$$

which is exothermic by $\sim 300 \mathrm{~kJ} \mathrm{~mol}^{1}{ }^{83}$ At 0.8 Torr Ar, a 10 ppm impurity of $\mathrm{H}_{2} \mathrm{O}$ gives a partial pressure of $8 \mu$ Torr, or $\sim 3 \times 10^{11} \mathrm{~cm}^{3}$. If reaction 3.15 (and/or reaction 3.17) occurs with collision efficiency, the reaction rate constant is expected to be $k_{\mathrm{ADO}} \varphi 2 \mathrm{x}$ $10^{-9} \mathrm{~cm}^{3} \mathrm{sec}^{-1}$. This rate was calculated using the Average Dipole Orientation (ADO) theory given by Su and Bowers, ${ }^{84}$ a C constant of $0.25,{ }^{84}$ and a polarizability and dipole for water of $1.45 \times 10^{-24} \mathrm{~cm}^{3}$ and 1.8546 Debye, respectively. ${ }^{85}$ Under these conditions, the reaction rate for reactions 3.15 (and/or 3.17) is $\sim 200 \mathrm{sec}^{-1}$.

The bond dissociation energy for $\mathrm{ArH}^{+}$is estimated to be $\sim 580 \mathrm{~kJ} \mathrm{~mol}^{1}$, or $\sim 6 \mathrm{eV},{ }^{83}$ and implies that the dissociative recombination of this species cannot populate any excited states of the neutral argon products. Therefore, kinetic energy and $\mathrm{H}$ excitation are the exclusive mechanisms by which energy is dissipated in the dissociation of the ArH molecule. Therefore, although contaminant gases may contribute to the loss of 
argon ions in the afterglow, this method cannot account for afterpeak emissions originating from levels above $14.47 \mathrm{eV}$ of $\mathrm{Ar}$ I.

As a final effort to understand the afterpeak emissions, we refer to the CRC model developed by Fujimoto ${ }^{36,37}$ (see Figure 3.8). At the electron densities expected in this study $\left(\sim 10^{11} \mathrm{~cm}^{-3}\right)$ the plasma passes through a very interesting phase in the CRC diagram: ${ }^{36,} 37$ the 'cascade' contribution dominates the 'capture' contribution' as a method of populating lower energy levels. For example, in hydrogen, radiative decay from the $\mathrm{p}>6$ levels is responsible for more than $90 \%$ of the populating mechanism for the $\mathrm{p}=5$ state. This implies that simple radiative decay from highly excited levels is increasingly important for the downward flow of energy exhibited in the afterglow. Also, because of a so-called bottle-neck effect ${ }^{33,36}$ caused by the slightly different de-excitation mechanisms above and below a critical quantum level (at p $\varphi$ 6), levels below this critical point will decay faster than levels above it. Remarkably, this phenomenon agrees, qualitatively at least, with the decay rates measured in Figure 3.12, in which the lower levels $\left(E_{p}<13.5\right)$ decay at a slightly faster rate than the higher levels. These results suggest that the cascade contribution from highly excited states of Ar I are indeed responsible for the occurrence of the afterpeak observed.

In agreement with Bogaerts and Gijbels, ${ }^{30}$ we also find a disparity between the recombination rates observed in the PGD afterglow and the recombination rates predicted at an electron density of $10^{11} \mathrm{~cm}^{3}$. Assuming radiative (two-body) and three-body recombinations to be dominant, the electron density would have to be taken as at least two orders of magnitude larger than expected in order reconcile the calculated recombination rates with our measured decay rates for the argon atom lines. The 
measured rates would require electron densities in the range of $10^{14} \mathrm{~cm}^{-3}$ (implying $1 \%$ ionization of Ar). Because this is highly unlikely, we reach the same conclusions as Bogaerts and Gijbels at the beginning of this discussion: we cannot find justification for the observed results. As a final word on this matter, Mason et al. have recently performed some experiments with a flowing afterglow $\mathrm{GD},{ }^{86}$ and offered evidence for significant contributions from long-lived excited states of Argon. ${ }^{87-92}$ These neutral states are speculated to be high-lying Rydberg states or auto-ionizing region (i.e. above the IP of $\mathrm{Ar}$ I) between 27-34 eV. Mason proposed that these levels could contribute to metastable formation (at 11.55 and $11.72 \mathrm{eV}$ ) via radiative or collisional decay, and that the highlyexcited levels might be more quickly quenched by contaminant gases. The confirmation or refutation of the contributions of such states to the afterglow period of the PGD would certainly be an interesting future project.

The argument for CRC involving argon ions the afterglow is supported by many previous reports in the PGD literature. As Yan, et. Al. demonstrate, the afterglow emission intensities increase as the pulse width increases. ${ }^{70}$ Increasing the pulse width increases the number density of argon ions that are available for recombination in the afterglow. At pulse widths below $20 \mu$ s there is almost no noticeable afterglow for the Ar I or $\mathrm{Cu}$ I states. The afterpeak became clearly visible at pulse widths longer than $50 \mu \mathrm{s}$. This argument is supported by the observations of other groups, that peaks in afterglow emissions typically are not observed in discharges with pulse widths shorter than $50 \mu \mathrm{s},{ }^{8,9}$, ${ }^{30,93-97}$ unless considerably higher powers are used to generate more charged pairs. ${ }^{56}$ 


\subsection{Conclusions}

Characterization of the light emitted by excited argon and copper atoms and ions reveals the transient nature of the flow of energy in different regions of the plasma. Fast atom/ion excitation dominates the upward flow of energy to the lower energy levels within 0-2 $\mathrm{mm}$ of the cathode during the voltage-on period, but this mechanism is not significant for the higher excited states or in the negative glow $(>3 \mathrm{~mm})$. Electron excitation governs the latter region during the pre-peak and steady-state regimes, but not to the same extent. The development of space-charge effects as the plasma lifetime increases drives the lower levels from the corona balance towards the saturation balance with the result that the lower levels are less populated in the steady-state than in the prepeak. The growth towards the saturation balance later in the pulse lifetime enhances the population of levels closer to the IP. Factors such as the initial state of the gas, the method of energy deposition in the plasma, the loss of charged particles to the walls, and the loss of radiation all contribute to deviations from TE and render the plasma in an ionizing state. The initial state of the gas is more influential at the pulse onset, which gives rise to the observed attenuation of higher lines and enhancement of lower lines.

Voltage termination results in electron thermalization and plasma recombination, demonstrated by the CRC flow of energy and population inversion in the afterglow. Levels closest to the IP become overpopulated with respect to TE conditions and cause a bottleneck effect in the downward flow of energy — meaning that the lower energy states depopulate more quickly than the higher energy states. Recombination effects are not observed close to the cathode, presumably because the electrons/ions diffuse to the cathode surface and are not available for recombination reactions. 
Disparate behavior is found between the decay rates of pptical emission lines measured here and the expected rates for recombination found in the literature. This difference can best be ameliorated by assuming a larger electron density (by three orders of magnitude) than expected for these conditions, or else assuming radiative- and threebody recombination rates to be in error. Alternatively, if high-energy Rydberg states or auto-ionizing states are available in sufficient numbers in the early afterglow, cascade contributions from these levels could presumably contribute to the observed afterglow, reducing the necessity for capture to take part in the CRC behavior of the afterglow.

Finally, the temporal emission studies have shown that intensities for different lines in optical emission determinations can be optimized under different conditions. The pre-peak tends to enhance the lower lying states (in the near-absence of emissions from high-lying states), while the afterglow amplifies upper-levelemissions. The afterglow causes a population inversion and further enhances emissions from levels closer to the IP of the neutral states. Atomic ion emissions are greatly attenuated in the afterglow because no method exists to populate the high-energy lines. 


\subsection{References}

(1) Chapman, B. Glow Discharge Processes; John Wiley and Sons: New York, 1980.

(2) Harrison, W. W.; Hess, K. R.; Markus, R. K.; King, F. L. Anal. Chem. 1986, 58, 341A-356A.

(3) Stuewer, D. Fresnius' J. Anal. Chem. 1990, 337, 737-742.

(4) King, F. L.; Harrison, W. W. Mass Spec. Rev. 1990, 9, 285-213.

(5) Marcus, R. K. Glow Discharge Spectroscopies, 1 ed.; Plenum Press, 1993.

(6) King, F. L.; Teng, J.; Steiner, R. E. J. Mass Spec. 1995, 30, 1061-1075.

(7) Barshick, C. M. In Inorganic Mass Spectrometry; Barshick, C. M., Duckworth, D. C., Smith, D. H., Eds.; Marcel Dekker, Inc.: New York, 2000; Vol. 23.

(8) Hang, W.; Walden, W. O.; Harrison, W. W. Anal. Chem. 1996, 68, 1148-1152.

(9) Bengston, A.; Yang, C.; Harrison, W. W. J. Anal. At. Spectrom. 2000, 15, 12791284.

(10) Hang, W.; Baker, C.; Smith, B. W.; Winefordner, J. D.; Harrison, W. W. J. Anal. At. Spectrom. 1997, 12, 143-149.

(11) Harrison, W. W.; Hang, W. Fres. J. Anal. Chem. 1996.

(12) Harrison, W. W.; Hang, W. J. Anal. At. Spectrom. 1996, 11, 835-840.

(13) Dawson, J. B.; Ellis, D. J. Spectrochim. Acta, Part A 1966, 23, 565-590.

(14) Boumans, P. W. J. M. Spectrochim. Acta, Part B 1991, 46, 711-739.

(15) Jackson, G. P.; Lewis, C. L.; Doorn, S.; Majidi, V.; King, F. L. Spectrochim. Acta, Part B 2001, 56, 2449-2464. 
(16) Lewis, C. L; Jackson, G. P.; Doorn, S. K.; Majidi, V.; King, F. L. Spectrochim. Acta, Part B 2001, 56, 487-501.

(17) Lewis, C. L. Dissertation, West Virginia University, Morgantown, 2001.

(18) Rozsa, K.; Gallagher, A. Phys. Rev. E 1995, 52, 913-918.

(19) Bogaerts, A.; Gijbels, R. Spectrochim. Acta, Part B 2000, 55, 279-297.

(20) Bogaerts, A.; Gijbels, R. Spectrochim. Acta, Part B 2000, 55, 263-278.

(21) Bogaerts, A.; Gijbels, R.; Vlcek, J. Spectrochim. Acta, Part B 1998, 53, 15171526.

(22) Bogaerts, A.; Gijbels, R. Spectrochim. Acta, Part B 1998, 53, 1-42.

(23) Bogaerts, A.; Gijbels, R. J. Am. Soc. Mass Spectrom. 1997, 8, 1021-1029.

(24) Ferreira, C. M.; Loureiro, J.; Ricard, A. J. Appl. Phys. 1985, 57, 82-90.

(25) Jelenkovic, B. M.; Phelps, A. V. J. Appl. Phys. 1999, 85, 7089-7096.

(26) Simpson, S. W. J. Phys. D: Appl. Phys. 1990, 22, 1161-1167.

(27) Vieth, W.; Huneke, J. C. Spectrochim. Acta, Part B 1990, 45, 941-949.

(28) Kano, K.; Suzuki, M.; Akatsuka, H. Plasma Sources Sci. Technol. 2000, 9, 314322.

(29) Budtz-Jorgensen, C. V.; Bottiger, J.; Kringhoj, P. Surf. Coat. Technol. 2001, 137, 104-110.

(30) Bogaerts, A.; Gijbels, R. J. Anal. At. Spectrom. 2001, 16, 239-249.

(31) Duncen, H.; Tiller, H. J.; Meisel, J. Spectrochim. Acta, Part B 1969, 24, 632-634.

(32) Strauss, J. A.; Ferreira, N. P.; Human, H. G. C. Spectrochim. Acta, Part B 1982, 37, 947-954.

(33) van der Mullen, J. A. M. Phys. Rev. 1990, 191, 109-220. 
(34) Fujimoto, T. J. Phys. Soc. Japan 1979, 47, 265-272.

(35) Fujimoto, T. J. Phys. Soc. Japan 1979, 47, 273-281.

(36) Fujimoto, T. J. Phys. Soc. Japan 1980, 49, 1569-1576.

(37) Fujimoto, T. J. Phys. Soc. Japan 1980, 49, 1561-1568.

(38) Fujimoto, T. J. Phys. Soc. Japan 1985, 54, 2905-2914.

(39) Aparicio, J. A.; delVal, J. A.; Gonzalez, V. R.; Gigosas, M. A.; Perez, C.; de la Rosa, I.; Mar, S. J. Phys. Soc. Japan 1999, 68, 3885-3892.

(40) King, F. L.; Pan, C. Anal. Chem. 1993, 65, 735-739.

(41) Martin, W. C.; Fuhr, J. R.; Kelleher, D. E.; Musgrove, A.; Podobedova, L.; Reader, J.; Saloman, E. B.; Sansonetti, C. J.; Weise, W. L.; Mohr, P. J.; Olsen, K., version 2.0 ed.; National Institutes of Standards and Technology, Gaithersburg, MD, 2002; Vol. 2002.

(42) Dyke, R. S. V.; Johnson, C. E.; Shugart, H. A. Phys. Rev. A 1972, 5, 991.

(43) Phelps, A. V.; Molnar, J. P. Phys. Rev. 1953, 89, 1202-1208.

(44) Futch, A. H.; Grant, F. A. Phys. Rev. 1956, 104, 356-361.

(45) Timmermans, E. A. H.; Thomas, I. A. J.; Jonkers, J.; Hartgers, E.; vanderMullen, J. A. M.; Schram, D. C. Fresenius' J. Anal. Chem. 1998, 362, 440-446.

(46) Bydder, E. L.; Miller, G. P. Spectrochim. Acta, Part B 1988, 43, 819-829.

(47) Fey, F. H. A. G.; Stoffels, W. W.; Mullen, J. A. M. V. d.; Sijde, B. V. d.; Schram, D. C. Spectrochim. Acta, Part B 1991, 46, 885-900.

(48) Kenty, C. Phys. Rev. 1928, 32, 624-635.

(49) Klinger, J. A.; Barshick, C. M.; Harrison, W. W. Anal. Chem. 1991, 63, 25712576. 
(50) Biondi, M. A. Phys. Rev. 1963, 129, 1181-1188.

(51) Biondi, M. A. Phys. Rev. 1952, 88, 660-665.

(52) Bogaerts, A.; Gijbels, R. Phys. Rev. A 1995, 52, 3743-3751.

(53) Bogaerts, A.; Guenard, R. D.; Smith, B. W.; Winefordner, J. D.; Harrison, W. W.; Gijbels, R. Spectrochim. Acta, Part B 1997, 52, 219-229.

(54) Scott, D. A.; Phelps, A. V. Phys. Rev. A 1991, 43, 3043-3055.

(55) Bogaerts, A.; Gijbels, R.; Carman, R. J. Spectrochim. Acta, Part B 1998, 53, 1679-1703.

(56) Hirabayashi, A.; Nambu, Y.; Hasuo, M.; Fujimoto, T. Phys. Rev. A 1988, 37, 7782.

(57) Atkins, P. Physical Chemistry, Sixth ed.; W. H. Freeman and Company: New York, 1998.

(58) Pan, C.; King, F. L. Anal. Chem. 1993, 65, 3187-3193.

(59) Pan, C.; King, F. L. J. Am. Soc. Mass Spectrom. 1993, 4, 727-732.

(60) Pan, C.; King, F. L. Appl. Spectrosc. 1993, 47, 2096-2101.

(61) Brackett, J. M.; Mitchell, J. C.; Vickers, T. J. Appl. Spectrosc. 1984, 38, 136-140.

(62) Fang, D.; Marcus, R. K. Spectrochim. Acta, Part B 1991, 46, 983-1000.

(63) Mehs, D. M.; Niemczyk, T. M. Appl. Spec. 1981, 35, 66-69.

(64) Ohorodnik, S. K.; Harrison, W. W. J. Anal. At. Spectrom. 1994, 9, 991-996.

(65) Pollmann, D.; Ingeneri, K.; Harrison, W. W. J. Anal. At. Spectrom. 1996, 11, 849853.

(66) Tachibana, K.; Fukuda, K. Jpn. J. Appl. Phys. 1973, 12, 895-902.

(67) Hornbeck, J. A.; Molnar, J. P. Phys. Rev. 1951, 84, 621-625. 
(68) Barshick, C. M.; Smith, D. H.; Johnson, E.; King, F. L.; Bastug, T.; Fricke, B. Appl. Spec. 1995, 49, 885-889.

(69) Bispham, J. W. Proc. R. Soc. London A 1908, 8, 477-487.

(70) Yan, X.; Ingeneri, K.; Hang, W.; Harrison, W. W. J. Anal. At. Spectrom. 2001, $16,819-824$.

(71) Lewis, C. L.; Oxley, E. S.; Pan, C. K.; Steiner, R. E.; King, F. L. Anal. Chem. 1999, 71, 230-234.

(72) Steers, E. B. M.; Fielding, R. J. J. Anal. At. Spectrom. 1987, 2, 239-244.

(73) Farnsworth, P. B.; Wooley, A.; Omenetto, N.; Matveev, O. Spectrochim. Acta, Part B 1999, 54, 2143-2155.

(74) Wagatsuma, K.; Hirokawa, K. Spectrochim. Acta, Part B 1991, 46, 269-281.

(75) Massey, H. S. W.; Burhop, E. H. S. Electron and Ionic Impact Phenomena; Oxford University Press, 1952.

(76) Biondi, M. A.; Holstein, T. Phys. Rev. 1951, 82, 962-963.

(77) Shiu, Y. J.; Biondi, M. A. Phys. Rev. A 1978, 17, 868-872.

(78) Gaur, J. P.; Chanin, L. M. Phys. Rev. 1969, 182, 167-175.

(79) Cooper, R.; VanSonsbeek, R. J.; Bhave, R. N. J. Chem. Phys. 1993, 98, 383-389.

(80) Zamir, E.; Szoke, A.; Osgood, R. J. Chem. Phys 1976, 65, 4885.

(81) Guna, M.; Simons, L.; Hardy, K. Phys. Rev. A 1999, 60, 306-313.

(82) Okada, T.; Sugawara, M. Jpn. J. appl. Phys. 1995, 34, 5829-5835.

(83) Lias, S. G.; Bartmess, J. E.; Leibman, J. F.; Holmes, J. L.; Levin, R. D.; Maillard, W. G. J. Phys. Chem. Ref. Data 1988, 17, Suppl. 1.

(84) Su, T.; Bowers, M. T. Int. J. Mass Spectrom. Ion Physics 1973, 12, 347-356. 
(85) Lide, D. R., Ed. CRC Handbook of Chemistry and Physics., 81 ed.; CRC Press: New York, 2000.

(86) Mason, R. S.; Miller, P. D.; Mortimer, I. P. Phys. Rev. E 1997, 55, 7462-7472.

(87) Lefaivre, D.; Marmet, P. Int. J. Mass Spectrom. Ion Physics 1975, 18, 153-164.

(88) Veillette, P.; Marchand, P. Int. J. Mass Spectrom. Ion Physics 1975, 18, 165-178.

(89) Simpson, J. A.; Chamberlain, G. E.; Mielczarek, S. R. Phys. Rev. A 1965, 139, 1039-1041.

(90) Marchand, P.; Cardinal, J. Can. J. Phys. 1979, 57, 1624-1633.

(91) Hua, X.; Bai, J.; Wang, L. Chem. Phys. 2001, 270, 93-98.

(92) Pichanick, F. M. J.; Simpson, J. A. Phys. Rev. 1968, 168, 64-74.

(93) Oxley, E.; Yang, C.; Harrison, W. W. J. Anal. At. Spectrom. 2000, 15, 1241-1245.

(94) Piepmeier, E. H.; de Galan, L. Spectrochim. Acta, Part B 1975, 30, 263-279.

(95) Su, Y.; Yang, P.; Zhou, Z.; Wang, X.; Li, F.; Huang, B.; Ren, J.; Chen, M.; Ma, H.; Zhang, G. Spectrochim. Acta, Part B 1997, 53, 1413-1420.

(96) Yang, C.; Ingeneri, K.; Harrison, W. W. J. Anal. At. Spectrom. 1999, 14, 693-698.

(97) Yang, C.; Harrison, W. W. Spectrochim. Acta, Part B 2001, 56, 1195-1208. 


\section{Chapter 4}

\section{Effect of Nitrogen on an Argon Pulsed Glow Discharge}

\subsection{Introduction}

Chapter 3 established that the steady-state nature of a glow discharge source deviates considerably from Thermodynamic Equilibrium (TE) and is ionizing in nature. Furthermore, the initial breakdown of the gas is more-strongly ionizing than the steadystate plasma due to the initial conditions of the gas and the sudden increase in electron excitation. Power cessation enables the electrons to thermalize via elastic collisions with the bath gas, ${ }^{1}$ which leads to the capture-radiative-cascade (CRC) observed in the afterglow. $^{2-4}$ This understand ing applies best to an ideal plasma sustained under relatively pure conditions. To achieve these conditions one requires clean sample-handling techniques, pure discharge gases, ${ }^{5}$ a leak-proof discharge chamber, and a long, lowpressure pump-down time to remove adsorbed gases from the internal surfaces. A cryogenic coil ${ }^{6-10}$ may also aid the elimination of contaminant water from the discharge. In real-world applications, analyses are often urgently undertaken and the analyst cannot afford to wait for the chamber to evacuate for long periods. In these circumstances, significant contaminant gases can be present in the discharge and will greatly affect the plasma characteristics. The effects of water on glow discharge mass spectrometry (GDMS) ion signals has been studied in detail, ${ }^{6,8,10-14}$ but the effect of nitrogen-the main constituent of atmospheric gas—is less well known. Wagatsuma and coworkers

have studied the effects of $\mathrm{N}_{2},{ }^{15-17}$ and other gases, ${ }^{18-20}$ in GD optical emission 
spectroscopy (GD-OES), but their work did not extend as extensively to mass spectrometric studies. ${ }^{15}$ The object of this study is to understand the effects of nitrogen contamination on plasma characteristics, and especially the effects on analyte ion signals for mass spectrometry.

Nitrogen also has some additional benefits as a tool for probing the plasma properties. For example, observation of the rovibrational emission spectrum elucidates a rotational temperature, which is a good indication of the gas temperature, ${ }^{9,}{ }^{21}$ and collisions between $\mathrm{N}_{2}$ and metastable states of argon result in the population of specific vibrational states of $\mathrm{N}_{2}{ }^{22-26}$ Emissions from these vibrational states reflect the relative populations of the two metastable levels at 11.55 and $11.72 \mathrm{eV}{ }^{23,25}$ This serves as an interesting diagnostic tool because of the importance of the metastables in GDMS.

The significance of metastables in GDMS was highlighted in the early part of the 1970's when Coburn and Kay developed a mass spectrometer to determine the kinetic energy of different species impinging on the anode of a steady-state ( $\mathrm{rf}$ and $\mathrm{dc}$ ) GD source. ${ }^{11,27}$ Careful examination of the ionized species using argon and neon as the discharge gas revealed the importance of the metastable states of the discharge gas in ionization processes. $^{27}$ Also, the fact that copper ion signals (determined by mass spectrometry) scaled linearly with the product of the copper atom and neon metastable populations (determined by atomic absorbance spectroscopy (AAS)) provided considerable evidence for the role of Penning Ionization. ${ }^{28}$ Following these initial studies, glow discharge mass spectrometry (GDMS) developed into a reliable technique for the bulk and trace analysis of conducting and non-conducting solids. ${ }^{29-37}$ A great wealth of research supports the observations of Coburn and Kay-that the metastables are 
responsible for a large proportion of the sputtered atom ionization in steady-state GDMS. $^{38,39}$ For correctness, it should be noted that Coburn and Kay were not the first to recognize the importance of Penning ionization in low-pressure plasmas, ${ }^{12,40-44}$ but their careful studies were one of the first to elucidate the role of these metastable species in ionizing sputtered atoms. This was an important breakthrough for GDMS applied in the analysis of solid samples.

In recent applications, microsecond ${ }^{45-47}$ and millisecond ${ }^{48-54}$ pulsed glow discharges (PGDs) have provided enhanced ionization efficiencies, and analyte ion signals temporally resolved from the discharge gas species (usually argon). This latter benefit is also highly dependent on the Penning ionization of sputtered atoms following termination of the plasma sustaining voltage. ${ }^{55}$ This has been demonstrated by quenching experiments using methane to prevent the metastables from taking part in the afterglow

processes. ${ }^{56}$ This chapter covers investigations into the effects of small quantities of molecular nitrogen in an argon PGD, with emphasis given to MS analysis. Optical experiments elucidate many of the effects of $\mathrm{N}_{2}$ addition on ion and excited-state formation—especially in the recombining afterglow—and are applicable to GD-OES and GD-AAS analyses. Time of flight mass spectrometry (ToF-MS) provides insight into the role of metastable argon atoms on analyte signals and persistent signals such as $\mathrm{ArH}^{+}$, $\mathrm{Ar}_{2}{ }^{+}$, and $\mathrm{Ar}^{2+}$.

\subsection{Experimental}

A $4.5 \mathrm{~cm}$ diameter iron disk (SRM 1767) served as the cathode sample throughout these experiments. Before taking data, a pre-sputtering time of $>30$ min ensured the 
removal of oxide/impurity residues from the cathode surface. Before this pre-sputtering period, the six-way cross was pumped down to below $10^{-4}$ Torr for several hours. The system was not checked for leaks and the ultra high purity argon (Airgas) was not further purified before use. Water impurities are assumed to be in the range of $10 \mathrm{ppm}$. A leak valve (Granville Phillips, Boulder, $\mathrm{CO}$ ) controlled the addition of nitrogen to the argon gas line. A pressure gauge (Hastings, Hampton VA) measured the partial pressure of nitrogen before and after each experiment. A second leak valve enabled the controlled addition of UHP argon to generate a total pressure of 0.8 Torr. Unless otherwise stated the nitrogen partial pressure equaled 0.01 Torr ( 1\% by volume).

\subsubsection{Optical experiments}

A schematic of the optical spectrometry system is provided in Figure 2.1. and is described in Chapter 2 and elsewhere. ${ }^{52,53,57}$ The hardware used here is identical to that provided in Chapter 2, with the exception of the square-wave-generating system that drives the glow discharge. In this study, an electrical chopper (GRX 3000, DEI) modulated the glow discharge power supply (OPS-3500, Kepco, Flushing, NY). The electrical chopper uses a square wave produced by a frequency generator (DS 345, Stanford Research Systems) to modulate the voltage. This pulsed voltage system provides a square-wave with rise and fall times less than $45 \mathrm{~ns}$. The homemade pulse generator used in the optical experiments in Chapter 2 has a fall time $V_{1 / 2}=220 \mu$ sec.

For temporal emission measurements at a fixed wavelength, the monochromator maintained a constant position while the digital oscilloscope (9370 M, Lecroy) recorded the output generated from the photomultiplier tube. $50 \Omega$ termination was used at the 
oscilloscope input. For temporal absorption measurements a lock-in-amplifier, (EG\&G PAR 5210, Princeton, NJ) linked to the chopper of the incident light beam, provided the oscilloscope with the amplified signal, as described in Chapter 2. An argon/iron hollow cathode lamp provided the incident beam. Deconvolution of the absorption data enabled the removal of most of the effects of the RC time constant in the lock-in-amplifier (See appendix in Chapter 2). To generate temporally resolved emission spectra, the output of the photomultiplier tube was fed into a boxcar as the monochromator was scanned. The monochromator and boxcar were synchronized so that the boxcar integrated a $1 \mu$ s gate width of signal from 10 consecutive pulses before the monochromator scanned to the next position. The boxcar therefore captures at least two data points per position of the grating. The gate could be shifted to any delay time from the onset of the glow discharge pulse to obtain only the emission spectra emitted during the $1 \mu$ s window at that delay time. This data acquisition setup is therefore able to monitor the emission lines of many different species at a specific time in the plasma.

\subsubsection{Time-of-Flight Mass Spectrometry (ToF-MS) experiments}

A schematic of the pulsed glow discharge time-of-flight mass spectrometry system is provided in Figure 4.1 and is described elsewhere. ${ }^{54}$ The power supply system included the electrical chopper described in section 4.2.1 and had a fall time considerably faster than the supply system used in Chapters 2 and 3. Operating parameters are given in Table 4.1. The packet of ions to be analyzed in the flight tube is extracted at right angles to the incident ion beam of the ion extraction lenses. This allows mass spectra to be collected at different times throughout the pulse cycle. A digital delay generator (4144, 
EG\&G, Princeton Applied Research) provided the delayed extraction pulses and was triggered by the same function generator that creates the glow-discharge pulse. Used in combination with the digital recording oscilloscope this arrangement enabled mass spectra to be obtained at selected temporal intervals with respect to the pulse trigger, in a similar manner described for the emission spectra above.

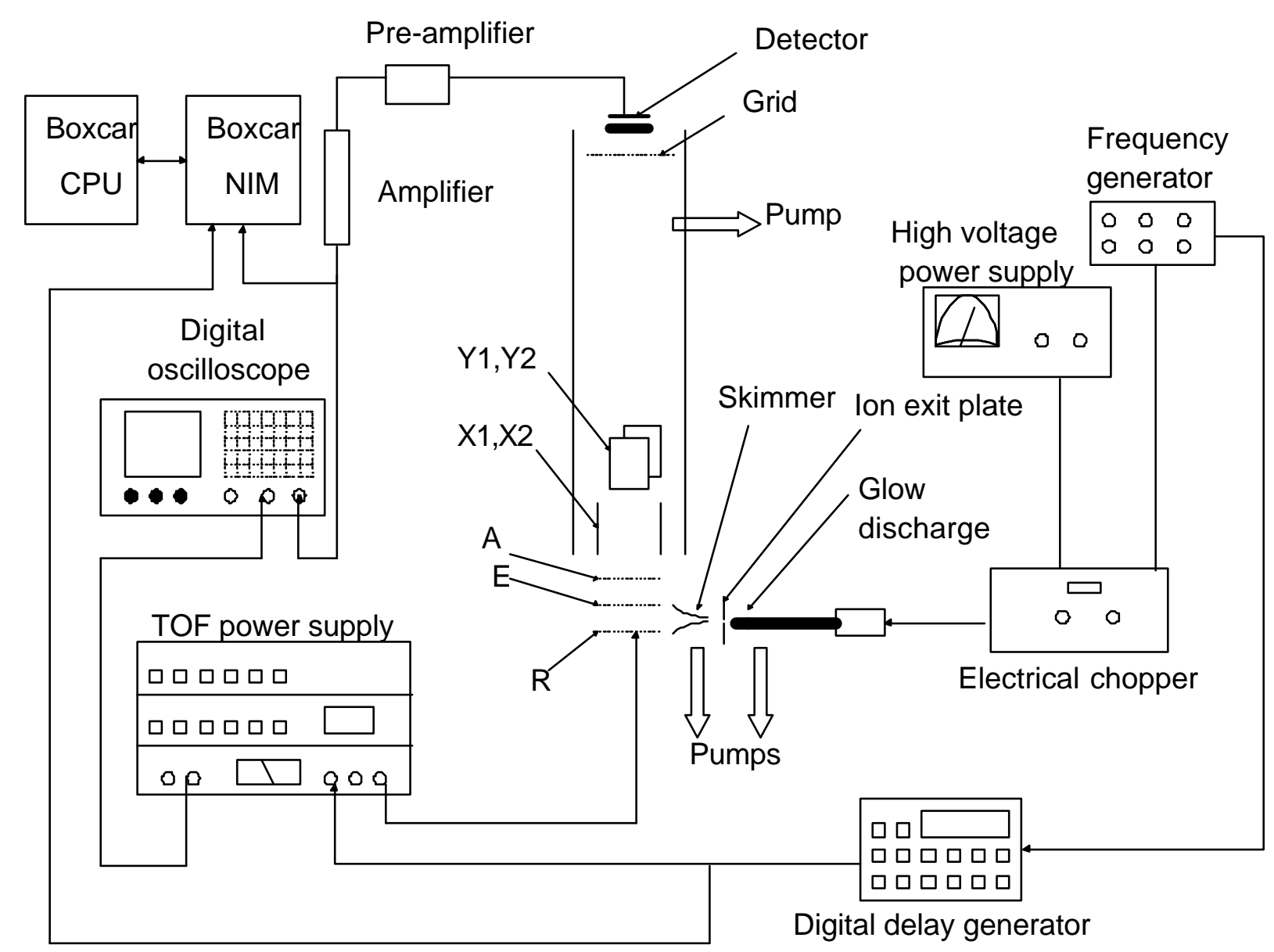

Fig. 4.1. Schematic of the Pulsed Glow Discharge Time-of-Flight Mass Spectrometer (PGD-ToF-MS) apparatus. 
Table 4.1. Settings used for the PGD-ToF-MS experiments.

Pressure (Torr)

$\begin{array}{cc}\text { GD Source } & 0.8 \\ \text { Ion exit plate }- \text { skimmer } & \sim 4 \times 10^{-4} \\ \text { Flight tube } & \sim 5 \times 10^{-6}\end{array}$

\section{Glow Discharge}

$\begin{array}{cc}\text { Peak current } & 0.4 \mathrm{~mA} \\ \text { Peak voltage } & -960 \mathrm{~V} \\ \text { Pulse length } & 5.0 \mathrm{~ms} \\ \text { Ion exit orifice } & 1.0 \mathrm{~mm} \\ \text { Skimmer cone orifice } & 1.0 \mathrm{~mm}\end{array}$

\section{TOF}

R; Repeller plate

$\begin{array}{cc}\text { Bias } & -8.5 \mathrm{~V} \\ \text { Pulse width } & 2 \mu \mathrm{s} \\ \text { Pulse voltage } & +200 \mathrm{~V} \\ \text { Extraction plate } & 0 \mathrm{~V}\end{array}$

A; Accelerator plate,

flight tube, grid, X2, Y2

$$
-1500 \mathrm{~V}
$$

$\mathrm{X} 1$

$-1850 \mathrm{~V}$

Y1

$-1550 \mathrm{~V}$

Detector

$-1700 \mathrm{~V}$ 


\subsection{Results and discussion}

\subsubsection{Bath gas temperature measurements}

The gas temperature $\mathrm{T}_{\mathrm{g}}$ can be determined by various passive techniques, i.e. emission spectroscopy, that do not disturb the plasma in any way. One method involves the measurement of the Doppler half-width of an emission line (or lines). ${ }^{58}$ This requires the use of a very high resolution instrument such as an interferometer. Using this technique Human and coworkers ${ }^{59,60}$ determined the gas temperature of a dc argon GD to be between $567-2873 \mathrm{~K}$. The temperature was linearly related to the discharge current (at constant voltage) and was affected by the cathode composition. This latter observation was thought to be linked to the fact that the sputtered samples constitute a significant fraction of the plasma composition and will therefore affect its properties.

Piepmeier and De Galan ${ }^{61}$ showed that by making Doppler measurements of a pulsed glow discharge end-on to the cathode surface, the emission spectra were blueshifted during the first $20 \mu$ s of the pulse. The blue shift decreased after this time. This implied that during the first $20 \mu$ s of the pulsed discharge sputtered species leave the cathode surface with considerably more kinetic energy than later in the pulse (or in steady state conditions). The methods proposed for this kinetic shift are i) ordinary diffusion and ii) convective ejection. Diffusion would be faster at the onset of the pulse because sputtered species have not yet entered the gas phase and the concentration gradient is larger. Convective ejection is described as the thermal gas expansion caused by a sudden temperature increase at the cathode surface. Although absolute temperatures were not calculated, the gas temperature is clearly larger at the beginning of the pulse than after 25 $\mu \mathrm{s}$. 
An alternative method to measure the gas temperature is to monitor the rotational emission from a (contaminant) molecule in the plasma. Rotational temperature measurements, $\mathrm{T}_{\text {rot }}$, were made by Harrison and coworkers using glow discharges with pulse widths ranging from $10 \mu$ s to the steady state. ${ }^{9,21}$ At a pressure of 1.5 Torr argon, the rotational temperatures were $\sim 400 \mathrm{~K}$ at the shortest pulse widths, increasing to $\sim 600$ $\mathrm{K}$ in a steady state dc plasma. ${ }^{21}$ The temperatures decreased with increasing distance from the cathode. At 1 Torr, the rotational temperatures decreased over the range 600$500 \mathrm{~K}$ from 0-2.5 mm above the cathode. ${ }^{9,21}$

The rotational temperature of $\mathrm{N}_{2}{ }^{+}$in the pulsed glow discharge is also determined in this study for a 0.8 Torr argon plasma with $\sim 1 \% \mathrm{~N}_{2}$ (vol.). An emission spectrum obtained for the first negative band of $\mathrm{N}_{2}{ }^{+}\left(\mathrm{B}^{2} \Sigma_{\mathrm{u}}{ }^{+} \rightarrow \mathrm{X}^{2} \Sigma_{\mathrm{g}}{ }^{+}\right)$is shown in Figure 4.2.a. This spectrum was collected on a pc via an $\mathrm{AD}$ converter attached to the photomultiplier tube and is therefore a time-averaged spectrum. The monochromator was scanned at $0.05 \mathrm{~nm}$ increments with an integration time of 0.5 seconds point ${ }^{-1}$ so the emission intensity was integrated over approximately 25 pulse cycles. Following the methodology provided by Harrison et al., 9,21 the peak intensities of the even $\mathrm{R}$ branch of lines were used to generate a Boltzmann plot of $a \mathrm{I} / 2(\mathrm{~K}$ ” +1$)$ versus $\left(\mathrm{K}^{\prime}+1\right)(\mathrm{K}$ ”+2), as demonstrated in Figure 4.2.b., where $a$ is the constant 2 for the even numbers of $\mathrm{R}, \mathrm{I}$ is the intensity of the line and $\mathrm{K}$ " is the rotational quantum number. The slope of this line is equal to $-\mathrm{B} h c / k \mathrm{~T}_{\text {rot }}$ where $\mathrm{B}$ is the rotational constant of the vibrational level, $h$ is the Planck constant, $c$ is the speed of light, $k$ is the Boltzmann constant and $\mathrm{T}_{\text {rot }}$ is the rotational temperature. Substituting in the values of the constants, the slope is equal to $-1.296 / \mathrm{T}_{\text {rot }}$, giving a temperature of 730 $\mathrm{K}$ at $5 \mathrm{~mm}$ above the cathode. This temperature is considerably higher than the largest 
rotational temperatures reported previously (of 500-600 K). ${ }^{21}$ However, this study uses a larger pulse power than the previous study, and a lower nitrogen partial pressure. It has been shown that partial pressure of nitrogen can have a significant effect on the temperature measurements (with higher temperatures measured at lower partial pressures of $\left.\mathrm{N}_{2}\right){ }^{9}$

A boxcar integrator, used in conjunction with the monochromator, obtained temperature measurements at different periods of the $5 \mathrm{~ms}$ pulse, as described in the experimental section. Emission spectra collected before $2 \mathrm{~ms}$ and after $5 \mathrm{~ms}$ did not contain sufficient signal intensity to be able to calculate reliable temperatures. Between 2-5 $\mathrm{ms}$ the temperatures were not significantly different from the time-averaged spectra of $720-850 \mathrm{~K}$. Gas temperatures in the prepeak would be expected to increase from the initial room temperature conditions to the $\sim 800 \mathrm{~K}$ temperatures observed, but evidence for this conjecture could not be provided using this approach. After performing these experiments, it was discovered that the reason that the temperature increase could not be witnessed using the rovibrational temperature is because it actually takes $>2 \mathrm{~ms}$ for the vibrational levels of $\mathrm{N}_{2}$ to equilibrate with the gas temperature at the pulse onset. ${ }^{62}$ 

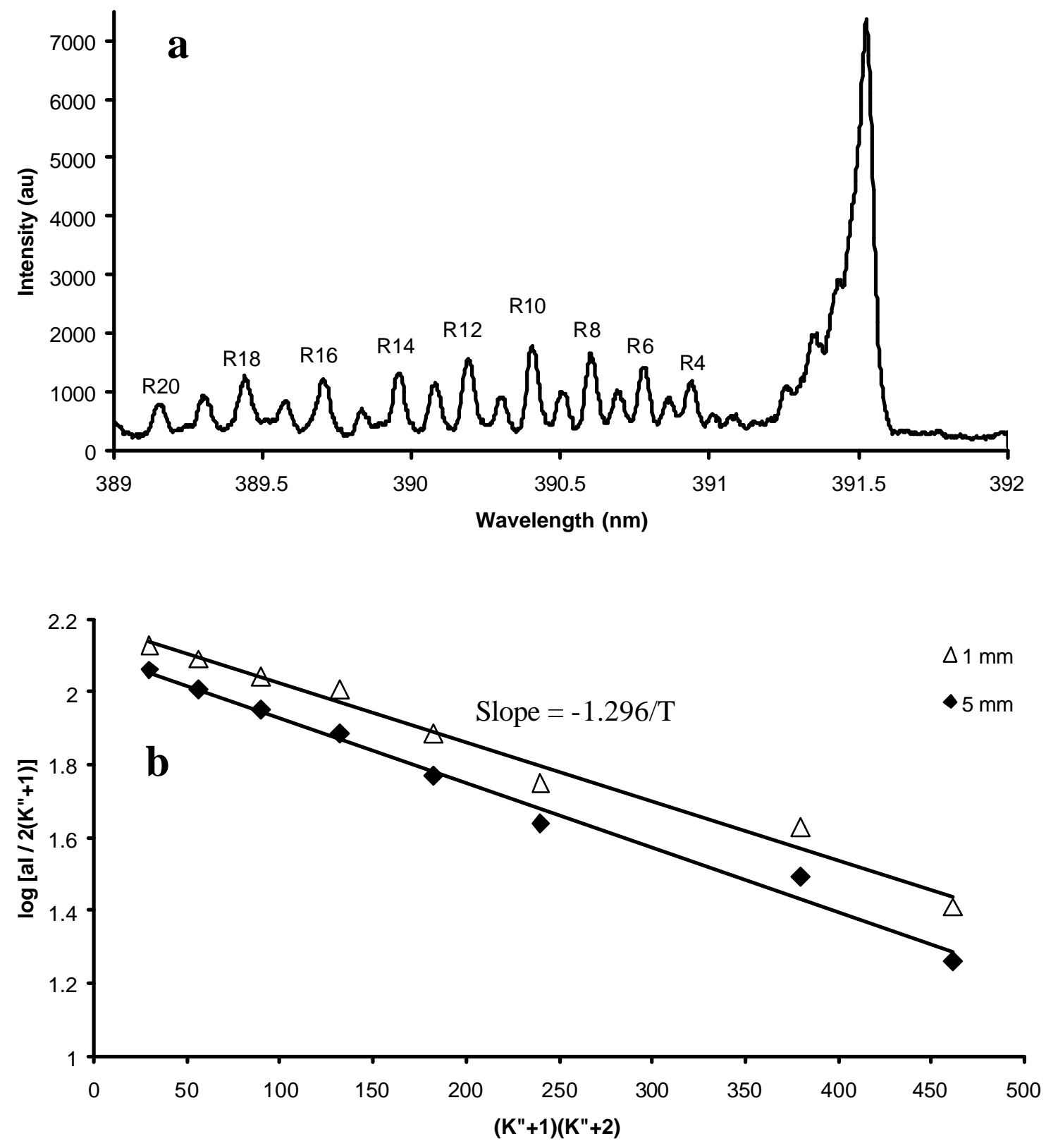

Fig. 4.2. a) Time-averaged emission spectrum of the first negative column of $\mathrm{N}_{2}{ }^{+}$in a PGD. Spectrum shown was taken at $5 \mathrm{~mm}$ above the cathode surface. b) Boltzmann plot for the determination of $\mathrm{T}_{\text {rot }}$ from the emission spectrum. $1 \% \mathrm{~N}_{2}$ in $0.8 \mathrm{Torr} \mathrm{Ar}, 1.5 \mathrm{~W}$ peak power. 
Figure 4.3 shows the time-averaged rotational temperature calculated at different distances above the cathode. It is found that the gas temperature decreases as the distance from the cathode increases, as described previously. ${ }^{9,} 21$ Two main factors contribute to the increased temperature near the cathode: i) argon ions accelerate across the cathode fall and charge exchange with argon atoms to create fast argon atoms; and ii) the sputtering process releases fast atoms and ions. ${ }^{30,36}$ These two effects generate atoms and ions with elevated kinetic energies and subsequent elastic collisions efficiently redistribute this energy to the bulk plasma atoms, thereby increasing the temperature. Because the two methods of kinetic energy deposition are cathode-region specific, the gas temperature is greater here than elsewhere.

In steady state GDs it is also possible for the cathode to resistively heat as the current flows through the sample. The increased cathode temperature provides another method for the gas near the cathode to acquire additional kinetic energy. In the PGD, the cathode does not heat up as much (this is one benefit of pulsing the discharge) because the discharge is only on for a fraction of the time and the cathode cools between pulses. 


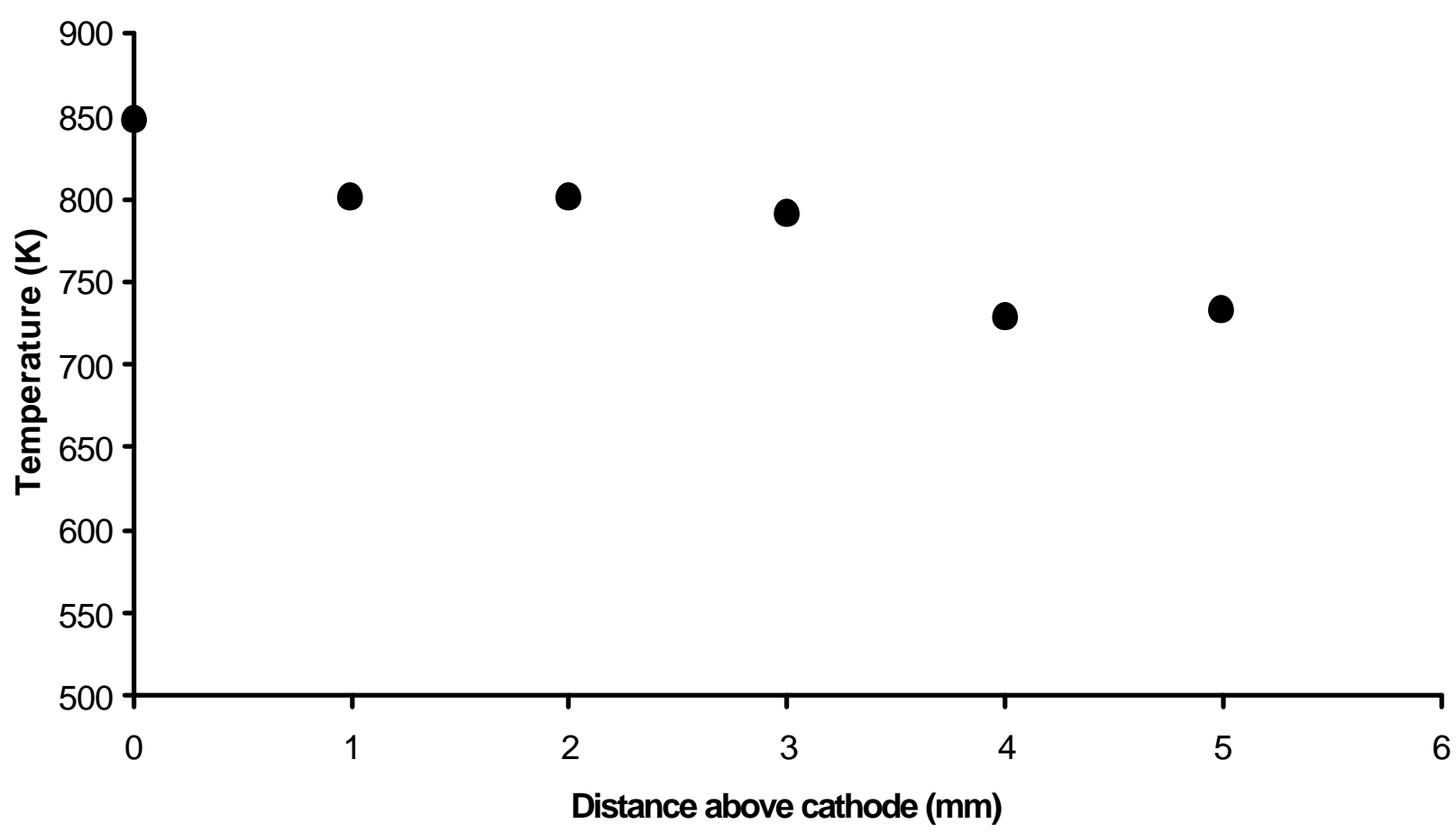

Fig. 4.3. Time-averaged rotational temperatures in the PGD determined from the emission spectra of $\mathrm{N}_{2}{ }^{+} .1 \% \mathrm{~N}_{2}$ in 0.8 Torr Ar, $1.5 \mathrm{~W}$ peak power. 


\subsection{2. $N_{2}$ quenching of metastable argon atoms}

In Chapter 2, temporal absorption profiles of the ${ }^{3} \mathrm{P}_{2}\left({ }^{1} \mathrm{~s}_{5}\right)$ and ${ }^{3} \mathrm{P}_{0}\left({ }^{1} \mathrm{~s}_{3}\right)$ metastable states of argon revealed differences in the post-pulse populations of these two states. Using a copper cathode, absorption at $811.5 \mathrm{~nm}$ for the ${ }^{3} \mathrm{P}_{2}$ state showed a considerably larger afterpeak than absorption at $794.8 \mathrm{~nm}$ for the ${ }^{3} \mathrm{P}_{0}$ state. The use of an iron cathode (here) revealed similar behavior. Phelps and Molnar also showed that in the afterpeak regime of a pulsed GD the lower energy ${ }^{3} \mathrm{P}_{2}$ state occupies a larger fraction of the total metastable population than during the steady-state regime. ${ }^{63}$ Stetser et al. showed that the higher energy ${ }^{3} \mathrm{P}_{0}$ state decays more rapidly than the lower metastable state, ${ }^{64}$ but no explanation was given to account for this behavior. A back-of-an-envelope calculation performed in Chapter 2 showed that during the capture-radiative-cascade (CRC) phase of the afterglow, the ${ }^{3} \mathrm{P}_{2}$ state has a larger probability of being formed than the ${ }^{3} \mathrm{P}_{0}$ state. The calculation involved summating the radiative transition probabilities (taking into account the degeneracy's of upper excited states) leading to the formation of each metastable state. This means that if the loss rates of the two states are comparable, the ${ }^{3} \mathrm{P}_{0}$ state will be lost more quickly because it has a lower probability of forming in the recombining plasma. In an ionizing or equilibrium plasma, the ${ }^{3} \mathrm{P}_{0}$ state will be populated from the ground state by electron excitation, and will form a larger fraction of the total metastable yield.

Considerable research has shown ${ }^{22-26}$ that electronic energy can be exchanged between the metastable states of argon and the electronic ground state of $\mathrm{N}_{2}$ in the reaction

$$
\operatorname{Ar}^{\mathrm{m}}+\mathrm{N}_{2}\left(\mathrm{X}^{1} \Sigma_{\mathrm{g}}^{+}\right) \rightarrow \mathrm{Ar}^{0}+\mathrm{N}_{2}\left(\mathrm{C}^{3} \Pi_{\mathrm{u}}\right)
$$


The electronically excited nitrogen molecule can also be vibrationally and rotationally excited in this reaction, as demonstrated by Figure 4.4. Both the metastable states can populate the $\mathrm{v}^{\prime}=0,1,2$ vibration levels, but only the ${ }^{3} \mathrm{P}_{0}$ metastable, at sufficient collision energy, can populate the $\mathrm{v}^{\prime}=3$ vibrational level. ${ }^{23,25}$ The reaction rate for (4.1) is $\sim 3.0 \mathrm{x}$ $10^{-11} \mathrm{~cm}^{3} \mathrm{~s}^{-1}$ for the ${ }^{3} \mathrm{P}_{2}$ state $^{26,6467}$ and $\sim 1.6 \times 10^{-11} \mathrm{~cm}^{3} \mathrm{~s}^{-1}$ for the ${ }^{3} \mathrm{P}_{0}$ state. ${ }^{66,68}$ Although the quenching rate of metastables by $\mathrm{N}_{2}$ is not particularly fast compared to other small molecules - the quenching rate for water is an order of magnitude faster ${ }^{68}$ - the number density of $\mathrm{N}_{2}$ at $\sim 3 \times 10^{14}$ gives quenching rates on the order of $\sim 10^{4} \mathrm{~s}^{-1}$. At typical number densities ${ }^{69,70}$ of $\mathrm{Ar}^{\mathrm{m}}$ of $\sim 10^{11} \mathrm{~cm}^{3} \mathrm{~s}^{-1}$ (without quenching), $\left[\mathrm{N}_{2}\right]>>\left[\mathrm{Ar}^{\mathrm{m}}\right]$ and the metastables should be efficiently quenched.

The excited molecular products radiatively decay with lifetimes of the second positive transitions $\left.\left(C^{3} \Pi_{\mathrm{u}}\right) \rightarrow \mathrm{B}^{3} \Pi_{\mathrm{g}}\right)$ on the order of $40 \mathrm{~ns}^{71,72}$ Given the number density of $\mathrm{N}_{2}$ and a bath gas temperature of $\sim 800 \mathrm{~K}$, Penning excitation of the nitrogen molecule will result in emissions from the nascent $\mathrm{C}^{3} \Pi_{\mathrm{u}}$ products of $\mathrm{N}_{2}$ before collisional mixing, or rovibrational relaxation can occur. ${ }^{22,25,64}$

Several methods exist to verify that excited states of $\mathrm{N}_{2}$ are formed via Penning excitation in the discharge, and not by fast atom or electron excitation. Two of the most distinguishing factors are i) that the $v^{\prime}=0$ vibrational of the $C^{3} \Pi_{u}$ level will be overpopulated with respect to the Frank-Condon probability factors in the presence of metastables, and ii) that the rotational fine structure of the $v^{\prime}=0$ will extend up to $K^{\prime} \approx 49$, independent of temperature. Also, because the metastables cannot populate above the $v^{\prime}=3$ level, emissions from these levels will not be observable. 


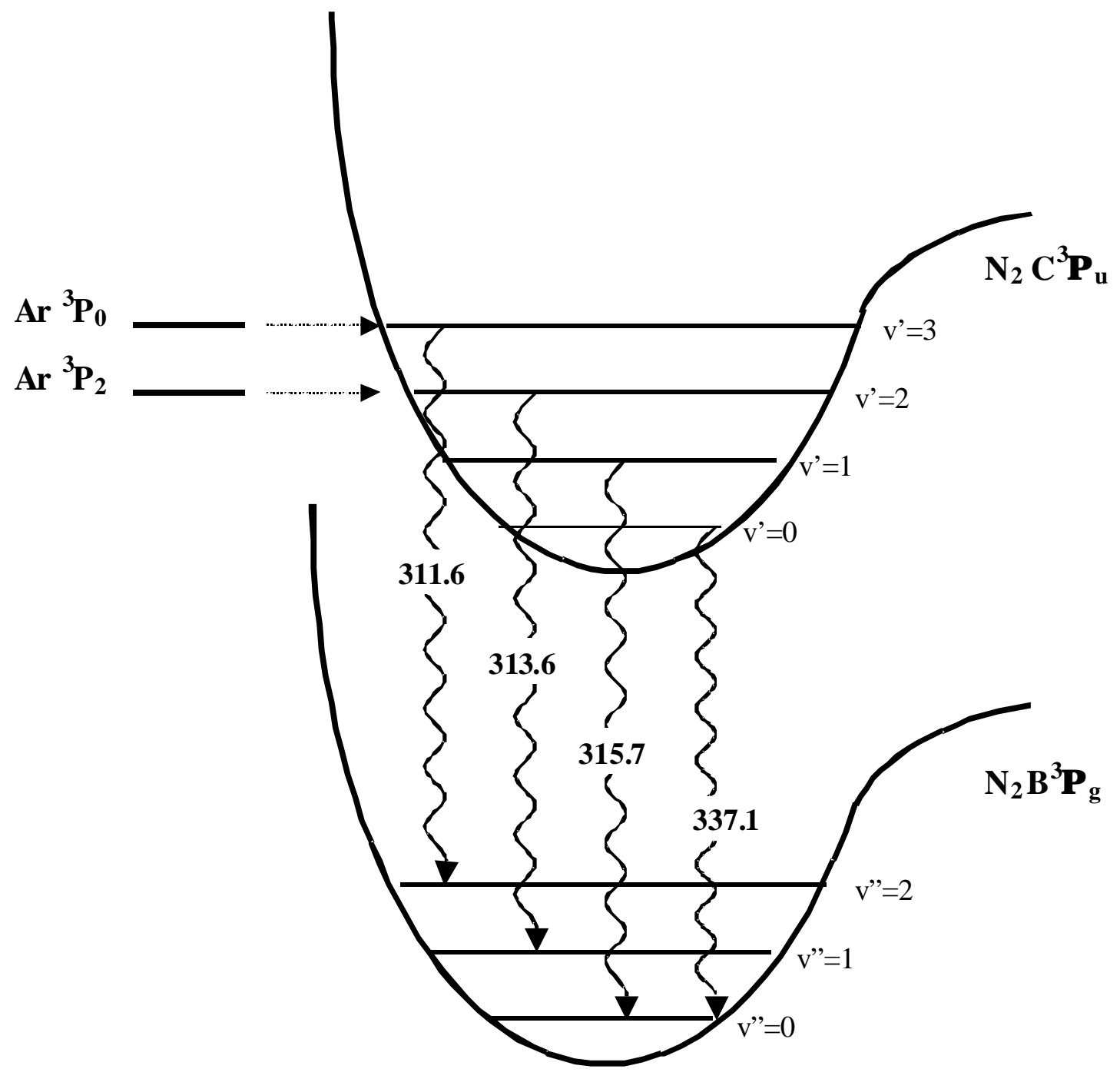

Fig. 4.4. Schematic to show the energy transfer reactions between argon metastables and molecular nitrogen, and the resulting emissions $(\mathrm{nm})$ used to characterize the quenching process. 
Figure 4.5 shows the rotational emission spectrum of the $(0,0)$ band of second positive emission with the band head at $337.1 \mathrm{~nm}$. The extensive excitation of higher rotational levels is clear evidence for the transfer of energy from metastable states of argon. To appreciate the deviation of these rotational levels from the Boltzmann distribution, contrast the spectrum in Figure 4.5 with the rotational spectrum of $\mathrm{N}_{2}{ }^{+}$in Figure 4.2.a. Emissions from vibrational levels $>v^{\prime}=3$ were not observed, also supporting that the excited states are predominantly formed by Penning excitation.

Figure 4.6 compares the steady-state emissions of the band-heads for various nitrogen molecular emissions as a function of distance above the cathode. The $(0,0)$ and $(2,1)$ band heads at 337.1 and $313.6 \mathrm{~nm}$, respectively, show very similar behavior with an emission maximum near the cathode surface. These values are self-consistent with energy transfer from the metastable states that also maximize at the cathode surface $38,39,73,74$ (see Chapter 2). The $(3,2)$ band emission, corresponding to energy transfer from the ${ }^{3} \mathrm{P}_{0}$ metastable (or possibly the resonant ${ }^{3} \mathrm{P}_{1}$ state) is less intense at the cathode surface relative to the negative glow region. This difference could reflect differences in the relative distributions of the metastable states, but because energy transfer from the resonant ${ }^{3} \mathrm{P}_{1}$ state cannot be ruled out, this conclusion cannot be verified. The molecular ion emissions at $391.3 \mathrm{~nm}$ shows considerably different behavior, having a maximum at the edge of the negative glow, at $2-3 \mathrm{~mm}$, and a minimum at the cathode surface. This level lies above the IP of argon, so the only mechanism of populating this state is via electron excitation. Because emissions from the second positive band show such different behavior, this is verification that the $C^{3} \Pi_{u}$ level is populated predominantly by the metastables. 


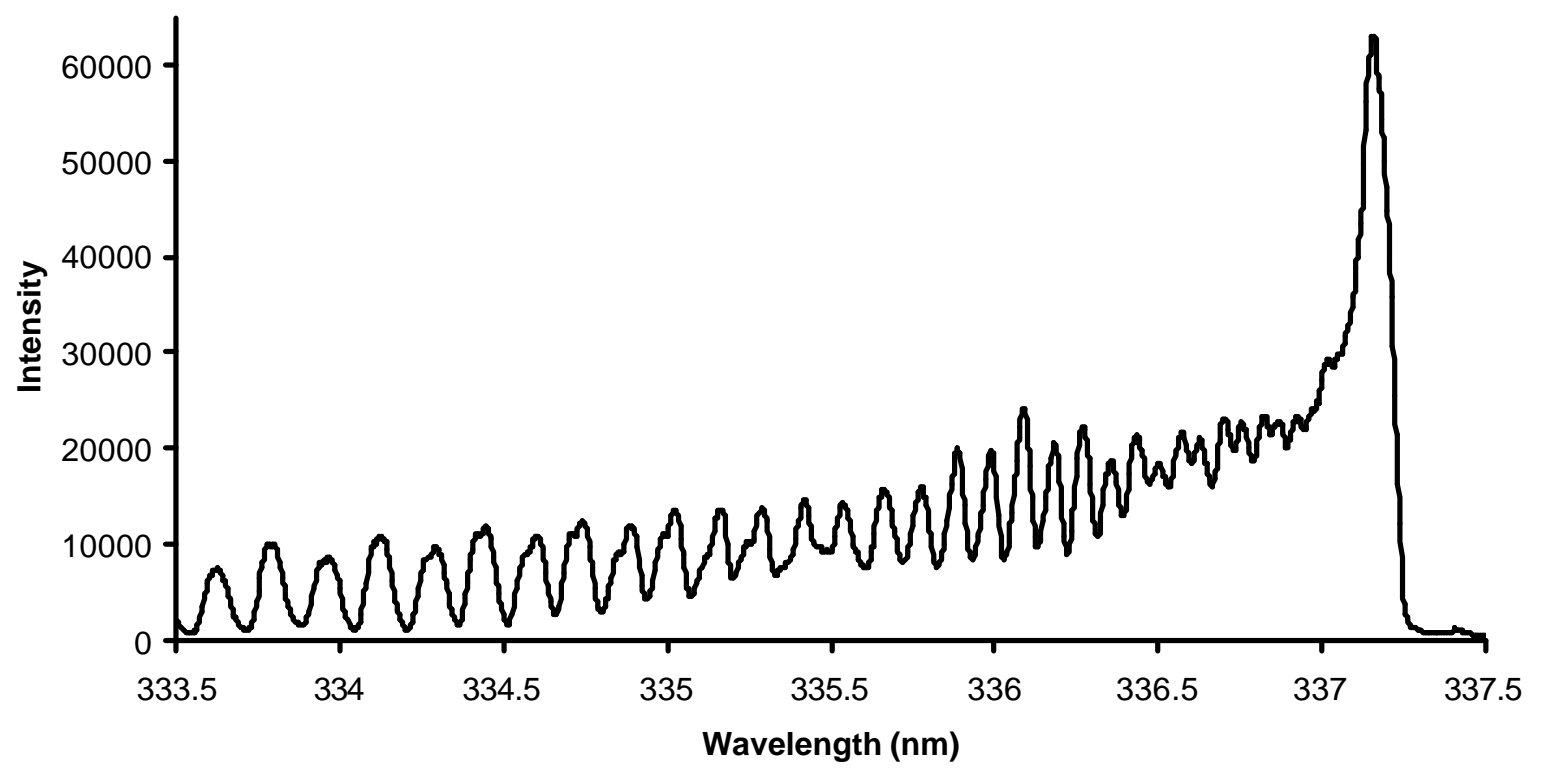

Fig. 4.5. Time-averaged rotational spectrum of the $(0,0)$ band of the second positive emission of nitrogen, with the band head at $337.1 \mathrm{~nm}$. The vast extent of rotational excitation is evidence for Penning excitation from argon metastables. $1 \% \mathrm{~N}_{2}$ by volume in 0.8 Torr Ar, $5 \mathrm{~ms}$ pulse width, $25 \%$ duty cycle, $\sim 1.5 \mathrm{~W}$ peak power.

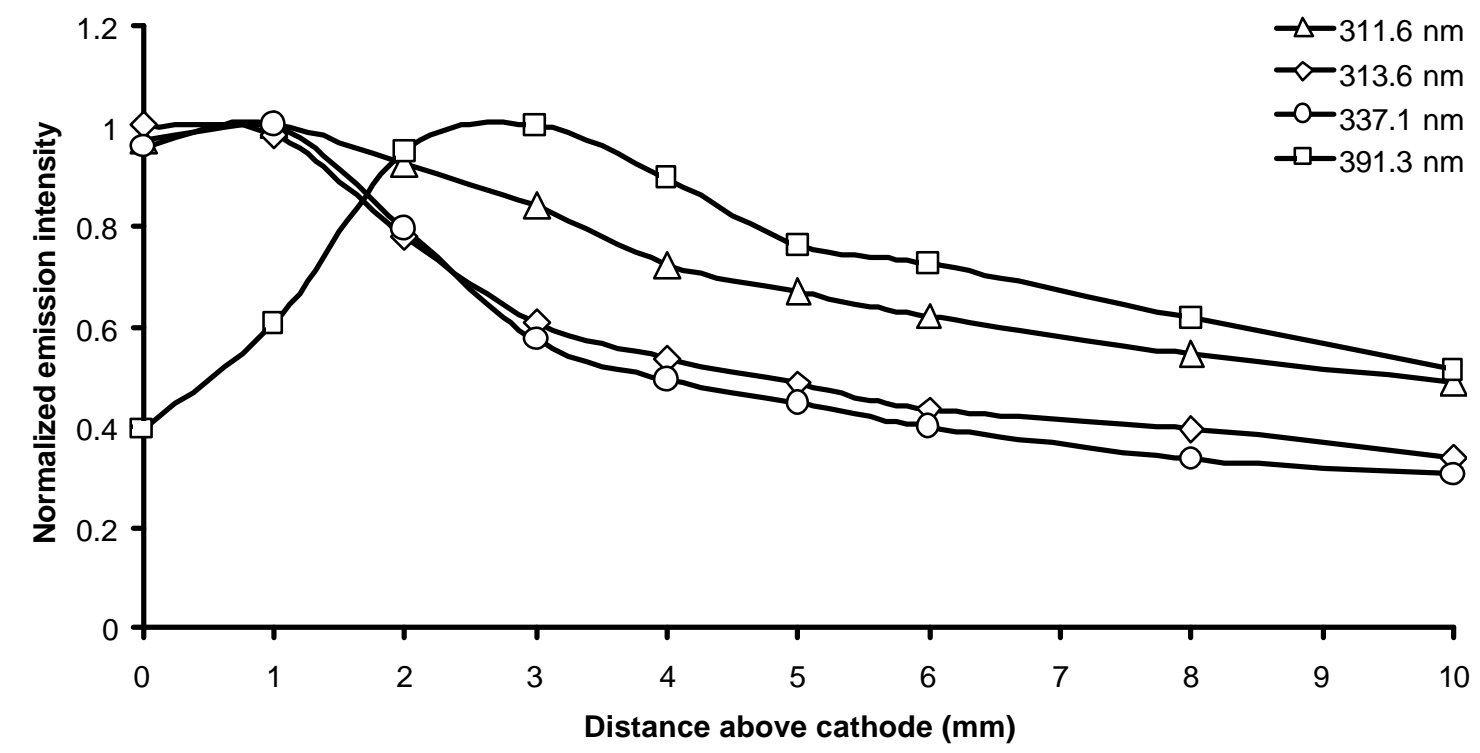

Fig. 4.6. Comparison of different nitrogen molecular emissions. Conditions given above. 
In addition to the time-averaged nitrogen molecular emissions described above, a fixed monochromator setting with the photomultiplier signal delivered to the digital oscilloscope enables temporal emission studies of the quenching effects. Figure 4.7 provides an example of the temporal emissions at $337.1 \mathrm{~nm}$ for the $(0,0)$ band of the second positive column.

Notice that there is no post-pulse increase in the emissions for this line, even though one would expect the metastable density to increase in afterglow. Chapters 2 and 3 showed that recombination is an effective way of increasing the number density of metastables in the afterglow period. The decay rates fr the second positive column emissions are only marginally slower than for the first negative column of the nitrogen molecular ion. If the nitrogen molecules quenched the metastables after they formed, the decay rate for the second positive system should be considerably longer than the first negative system of the $\mathrm{N}_{2}$ ion. Therefore, it is clear that the nitrogen must be preventing metastable formation in the afterglow—not simply quenching them after they form. Because a large portion of the afterglow metastable population is formed via captureradiative-cascade (CRC), experiments were performed to discern the effect of $\mathrm{N}_{2}$ on the afterglow processes.

Absorption measurements of the ${ }^{3} \mathrm{P}_{2}$ metastable states at $811.5 \mathrm{~nm}$ revealed that the afterpeak increase in metastables normally observed in pure argon PGDs is not observed with $1 \% \mathrm{~N} 2$ present. Figure 4.8 demonstrates this effect at $6 \mathrm{~mm}$ above the cathode. Closer to the cathode, post-pulse increases in metastables are not observed in pure argon, so nitrogen did not effect the decay rate of the metastables in this region. 


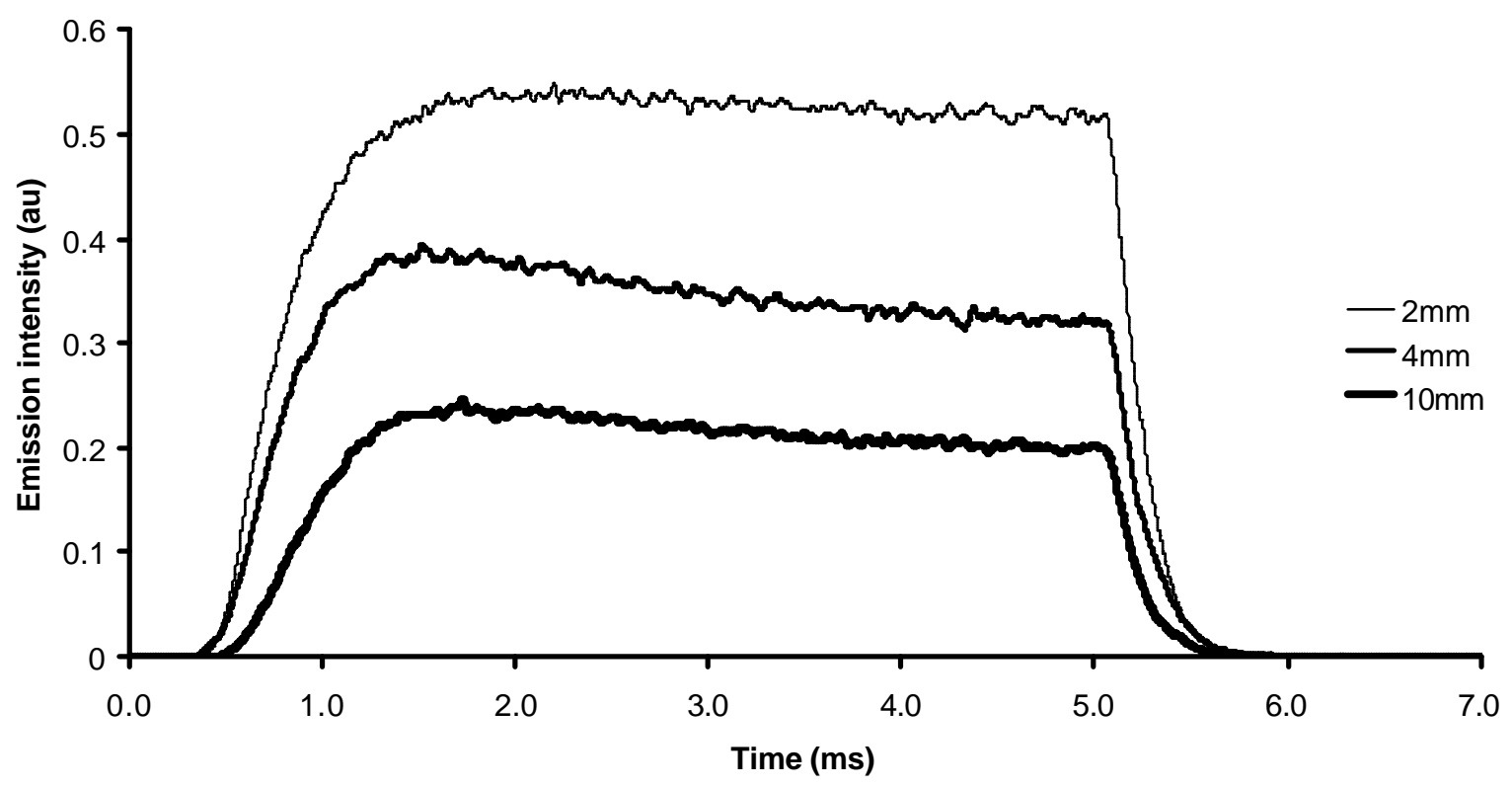

Fig. 4.7. Temporal emission profiles at $337.1 \mathrm{~nm}$ for the $(0,0)$ band of the second positive column. $1 \% \mathrm{~N}_{2}$ by volume in 0.8 Torr Ar, 5 ms pulse width, $25 \%$ duty cycle, $\sim 1.5$ $\mathrm{W}$ peak power.

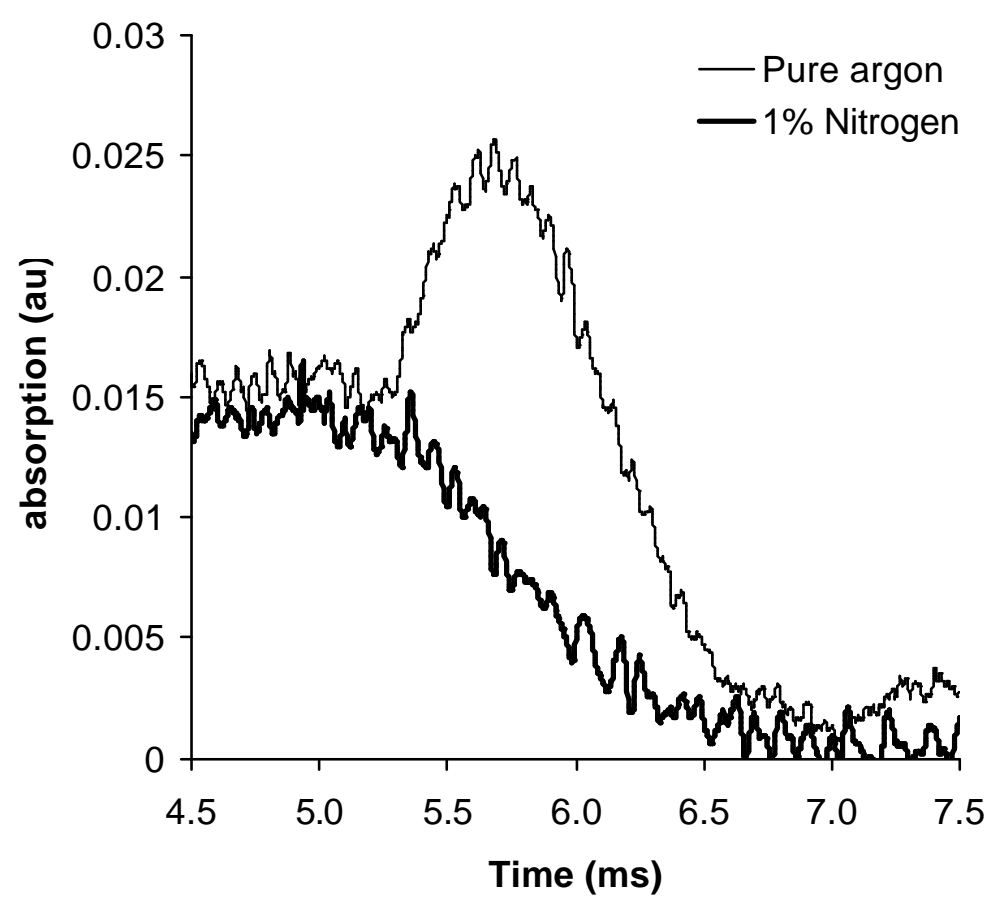

Fig. 4.8. Effect of nitrogen on the post-pulse population of ${ }^{3} \mathrm{P}_{2}$ metastables. Steady-state values have been normalized to the same value at $5.0 \mathrm{~ms}$. 
The absorption measurements show that there is no post-pulse increase in metastables when nitrogen is present, which explains why no post-pulse increase is observed for the second-positive-column emissions of $\mathrm{N}_{2}$. It should be noted that although deconvoluted data is shown in Figure 4.8 the process does not completely remove the effects of the RC time constants (see appendix in Chapter 2 for details on the deconvolution process). The afterpeak maximum therefore appears more quickly than shown in this figure.

Figure 4.9 shows the afterpeak/steady-state absorption ratio of the ${ }^{3} \mathrm{P}_{2}$ metastable populations plotted as a function of distance above the cathode. The relative populations were determined in a 'pure' argon discharge, and with $1 \% \mathrm{~N}_{2}$ added. When no post-pulse increase is observed, the absorption measurement at $5.5 \mathrm{~ms}$ is used. This is the time at which afterpeak signals reach a maximum when maxima are observed. A ratio greater than one, as seen for the ${ }^{3} \mathrm{P}_{2}$ state in pure argon, indicates a post-pulse increase in the population. Both metastables show an increase in the afterpeak/steady-state ratio as increasing distances from the cathode, but, as observed for the copper/argon plasma discussed in the previous two chapters, the afterpeak/steady-state ratios for the ${ }^{3} \mathrm{P}_{2}$ states are considerably larger than the ${ }^{3} \mathrm{P}_{0}$ state.

When nitrogen is admitted, there is no afterpeak increase in the number of metastables, as shown in Figure 4.8, and the ratio remains close to 0.5 at each sampling point. This shows that the decay rate is approximately equal at each distance, although the time constant inherent in the absorption measurements could mask any small changes in decay rates. 


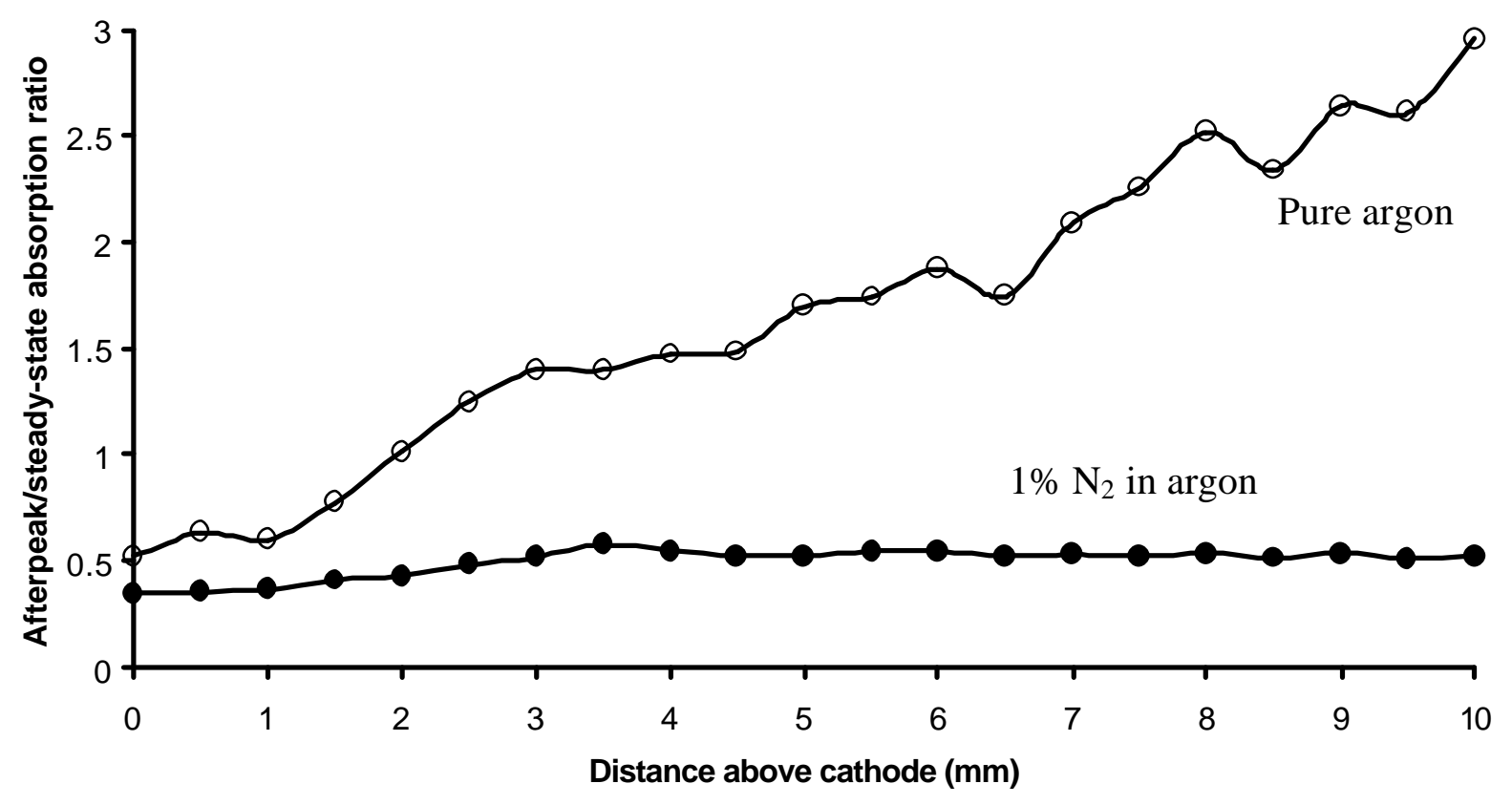

Fig. 4.9. Plot of the afterpeak/steady-state absorption ratios for the ${ }^{3} \mathrm{P}_{2}$ metastable levels of argon, before (filled markers) and after (open markers) the addition of $1 \% \mathrm{~N}_{2}$ to the 0.8 Torr argon plasma. Population ratios determined by absorption at 811.5 nm, pulse length $5.0 \mathrm{~ms}, 0.8$ Torr Ar, $\sim 1.5 \mathrm{~W}$ peak power, Fe cathode. 
The absorption measurements in Figures 4.8 and 4.9 show the effective removal of metastable atoms from the afterpeak, but these measurements do not reveal the exact nature of quenching, i.e. whether the metastables are quenched after they are formed, and/or if the metastable formation is prevented. As alluded to above, the $\mathrm{N}_{2}$ emissions indicate that metastables are prevented from forming rather than quenched once they are formed. Examination of the post-pulse emissions arising from different excited neutral atoms, indulged by this postulate, illuminates the dominant post-pulse quenching mechanism.

\subsection{3. $\mathrm{N}_{2}$ effects on post-pulse recombination}

In Chapter 3, considerable evidence elucidated the CRC behavior of the postpulse period of the PGD. The fundamental characteristic of the CRC plasma is the downward flow in energy from ion and excited states to the ground-state atoms. The switch from an ionizing plasma to a recombining plasma causes a population inversion and, because of the relatively low electron densities in these conditions, the decay process is predominantly radiative. Although the CRC ultimately ceases at the ground state levels, it is possible for the decay process to pause at the metastable levels because spinselection rules disallow their decay to the ground state. This is one reason why removal of the plasma-sustaining voltage ordinarily increases the number density of metastable states. 
Careful analysis shows that the added nitrogen deleteriously prevents the CRC process yielding an entirely different post-pulse outcome. Figures 4.10.a and 4.10.b show the effect of $1 \%$ nitrogen on the afterglow emission at $811.5 \mathrm{~nm}$ at 4 and $8 \mathrm{~mm}$ above the cathode, respectively. This emission corresponds to a $4 \mathrm{p}-4 \mathrm{~s}$ transition $\left(2 \mathrm{p}_{9}-1 \mathrm{~s}_{5}\right)$, as described in Chapter 2, and leads to the formation of the ${ }^{3} \mathrm{P}_{2}$ metastable state. At $4 \mathrm{~mm}$, $1 \%$ nitrogen has very little effect on the steady-state population of the upper $4 p$ state, as demonstrated by the similar emission intensities between 4-5 mm. Populations were even less effected closer to the cathode. Close to the cathode, the electron and fast atom collisions populating and depopulating the $4 \mathrm{p}$ (and $4 \mathrm{~s}$ ) states are extremely fast and outweigh any depopulation caused by the nitrogen. Further from the cathode, at $8 \mathrm{~mm}$ for example (Figure 4.10.b), the steady-state populations of the $4 p$ states are partially quenched by the added nitrogen. This is because excitation and de-excitation rates are slightly slower here, and stepwise electron excitation via the metastables is more prominent. ${ }^{75}$ Therefore, electronic energy transfer from excited argon states to nitrogen states is more noticeable here.

Figures 4.10.a and $\mathrm{b}$ show that when the voltage is removed from the pure argon plasma at $5.0 \mathrm{~ms}$, a post-pulse increase in emission ensues. In the presence of $1 \%$ nitrogen, this effect is almost completely absent. To answer an earlier question, these experiments show that the added nitrogen does prevent the metastables from forming in the afterglow, possibly in addition to quenching any that do form. There are two possible methods for $\mathrm{N}_{2}$ intervention in the CRC process: 1) the nitrogen reduces the capacity for argon ions to participate in recombination; and 2) the nitrogen reduces the capacity for electrons to participate in recombination. 

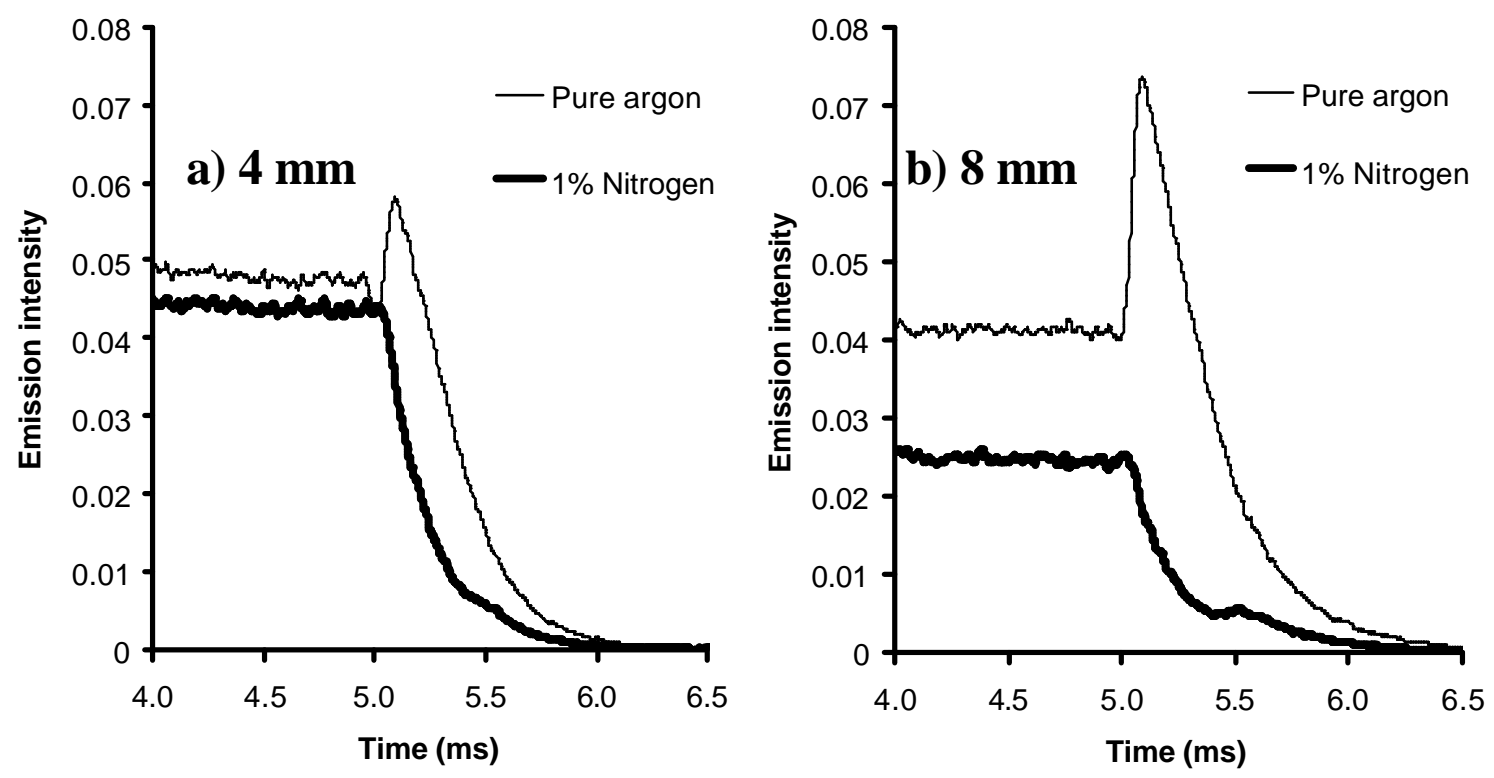

Fig. 4.10. Emission intensity at $811.5 \mathrm{~nm}$ versus time to show the effect of nitrogen on the afterpeak emissions in an argon PGD. 5.0 ms Pulse length, 25\% duty cycle, 0.8 Torr Ar, $1.5 \mathrm{~W}$ peak power, Fe cathode.

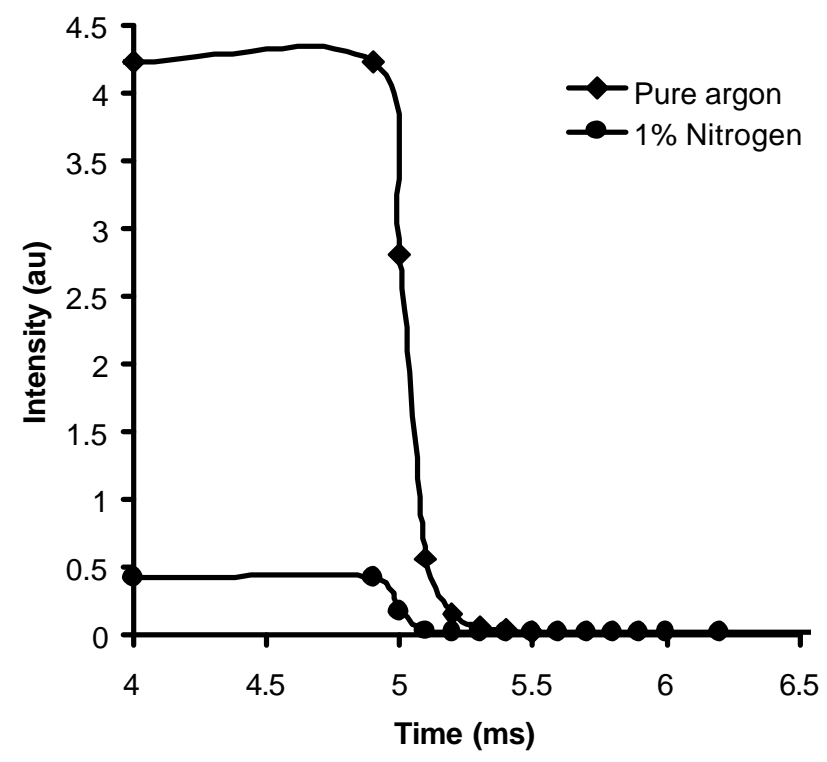

Fig. 4.11. Argon ion signal at $m / z=40$ versus time to show the effect of nitrogen on the steady-state and post-pulse number densities. Sampling orifice positioned at $5 \mathrm{~m}$ above the cathode, conditions given above. 


\subsubsection{Effect of $\mathrm{N}_{2}$ on argon ions}

To test the validity of the first possibility given above, the argon ion signal was observed via ToF-MS at different times during the pulse cycle. Figure 4.11 shows that in the steady-state plasma (observed between $4-5 \mathrm{~ms}$ ), the addition of $1 \%$ nitrogen reduced

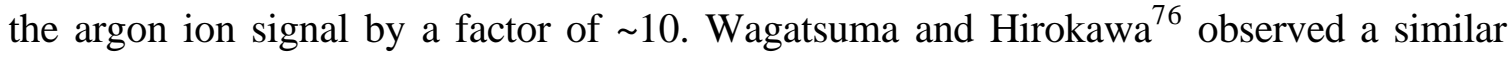
reduction in argon ions using a copper cathode. In Wagatsuma and Hirokawa's

experiment, ${ }^{76}$ the ground state argon ions were indirectly observed through the emission at $224.7 \mathrm{~nm}$, which is characteristic of charge exchange between $\mathrm{Ar}^{+}$and $\mathrm{Cu}^{0}$ (see Chapters 2 and 3). The reduction in argon ions here is thought to arise from charge transfer reactions with the nitrogen molecules ${ }^{77,78}$

$$
\operatorname{Ar}^{+}\left({ }^{2} \mathrm{P}_{1 / 2},{ }^{2} \mathrm{P}_{3 / 2}\right)+\mathrm{N}_{2}\left(\mathrm{X}^{1} \Sigma_{\mathrm{g}}^{+}\right) \rightarrow \operatorname{Ar}^{0}+\mathrm{N}_{2}^{+}\left(\mathrm{X}^{2} \Sigma_{\mathrm{g}}^{+}\right)
$$

followed by dissociative recombination of the nitrogen molecular ion with an electron.

$$
\mathrm{N}_{2}{ }^{+}+\mathrm{e}^{-} \rightarrow \mathrm{N}+\mathrm{N}^{*}
$$

Reaction (4.2) has a rate constant of $\sim 5 \times 10^{-11} \mathrm{~cm}^{3} \mathrm{~s}^{-1}$ (although the exact rate is somewhat pressure and electron-density dependent). ${ }^{77,78}$ Given a number density of $\mathrm{N}_{2}$ of $\sim 3 \times 10^{14} \mathrm{~cm}^{-3}\left(8 \times 10^{-3}\right.$ Torr) the quenching rate should be on the order of $\sim 10^{4} \mathrm{~s}^{-1}$. Indeed, using the $\mathrm{Ar}^{+}$signals in the lower plot of Figure 4.11 the loss rate of $\mathrm{Ar}^{+}$with $1 \%$ $\mathrm{N}_{2}$ present is calculated to be $\sim 10500 \mathrm{~s}^{-1}$, in excellent agreement with the predicted rate. Although the argon ion decay rate with $1 \%$ nitrogen was more than twice as fast as the rate without nitrogen, the argon ion loss rate in the 'pure' plasma is anomalously high. This phenomenon is discussed in detail in the latter sections of Chapter 3. Also discussed in Chapter 3, dissociative recombination, shown in equation 4.3, is typically three orders of magnitude faster than two-body radiative recombination. ${ }^{79,80}$ This implies that the 
added nitrogen offers a very fast method for plasma recombination to occur and, in the process, prevents argon ions from forming the highly excited neutral states normally observed in the afterglow.

\subsubsection{Effect of $\mathrm{N}_{2}$ on electrons}

The addition of $1 \%$ nitrogen to the plasma shows a reduction in the number density of argon ions and a reduction in the capacity for argon ion-electron recombination in the afterpeak. By varying the concentration of nitrogen, it is also possible to determine an electron-energy effect. Figure 4.12 shows the emission at $811.5 \mathrm{~nm}$ as a function of time at different partial pressures of nitrogen. The method for adjusting the partial pressures was to close the nitrogen valve and allow the vacuum pump to slowly remove the nitrogen contaminants. Before closing the valve, the plasma established the usual operating condition of $\sim 1$ mTorr ( $\sim 1 \%$ by volume) of $\mathrm{N}_{2}$ in the 0.8 Torr Ar plasma. While this experiment does not provide quantitative results, the observations that it does provide are extremely informative.

As nitrogen is pumped out of the discharge region, an afterpeak appears within the first minute. The peak is small and has a peak maximum later than $5.3 \mathrm{~ms}$. As more nitrogen is removed from the plasma, the afterpeak increases in intensity and appears at

decreasingly shorter times. After 15 minutes, the plasma resembles 'pure' conditions and the afterpeak maximum is close to $5.1 \mathrm{~ms}$. 


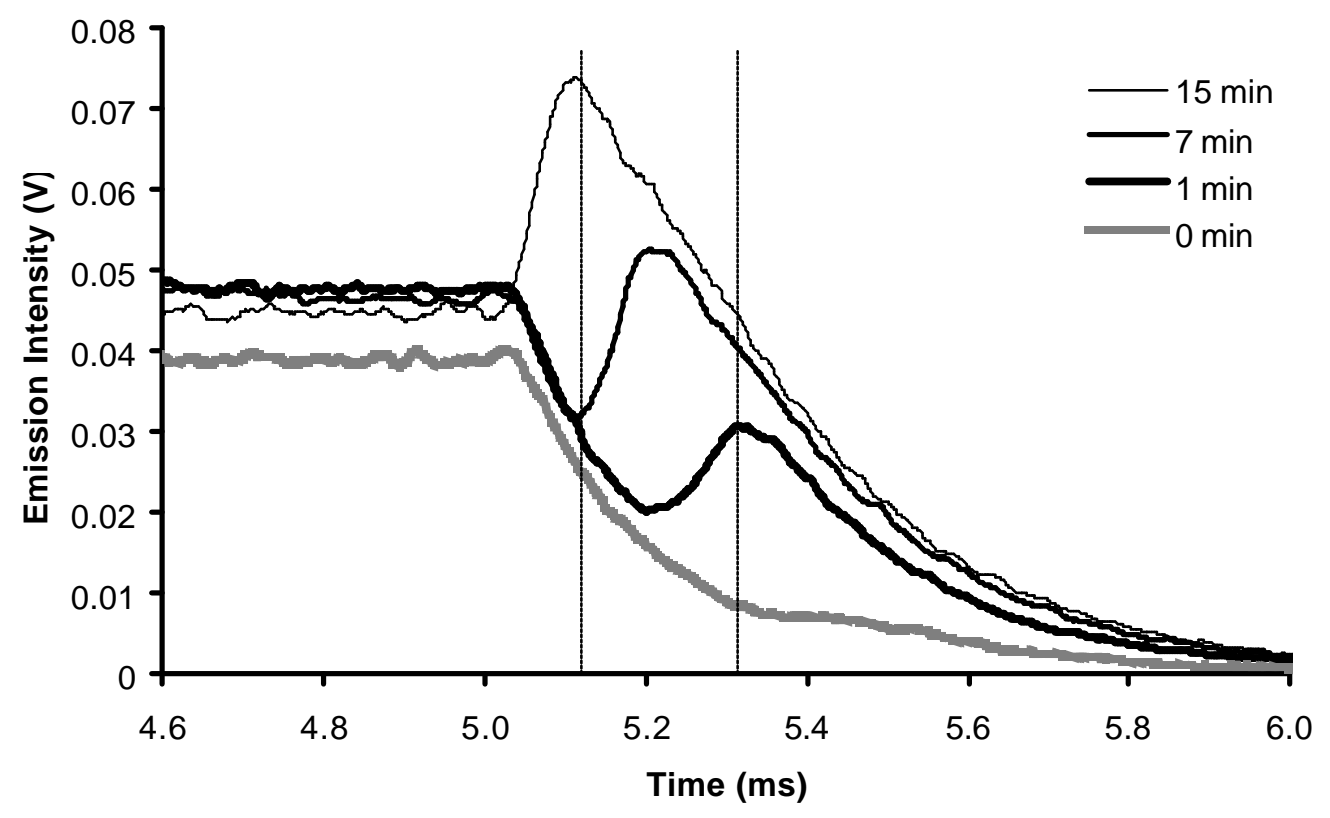

Fig. 4.12. Emission from the $811.5 \mathrm{~nm}$ line as a function of time to show the effect of decreasing nitrogen in the discharge. The times labeled in the figure refer to the time after the nitrogen valve was closed. Measurements made $5 \mathrm{~mm}$ above the cathode, 5.0 ms pulse length, $25 \%$ duty cycle, 0.8 Torr Ar, $1.5 \mathrm{~W}$ peak power, Fe cathode. 
Increasing the partial pressure of nitrogen has the unquestionable effect of delaying the appearance of the afterpeak, and in so doing, reduces the intensity of the emissions when they finally do appear. The number densities of argon ions was not determined in a comparative experiment, but we will assume that the steady-state values of $\left[\mathrm{Ar}^{+}\right]$will vary somewhat linearly with the nitrogen concentration between the two limits established in Figure 4.11. Charge transfer reactions between argon ions and nitrogen molecules could feasibly account for the difference in emission intensity in the afterglow, because increasing $\left[\mathrm{N}_{2}\right]$ would decrease the number of argon ions available for recombination. Although this mechanism can account for the intensity of the afterpeak emissions, it does not explain the delayed appearance at higher concentrations of $\mathrm{N}_{2}$.

Because recombination is inefficient until electrons reduce their kinetic energy to the gas temperature, a time delay between voltage termination and recombination will be observed while the electrons collisionally cool with bath gas atoms. ${ }^{79,} 81$ Although the addition of lighter, atomic ions can speed up this thermalization process, ${ }^{82}$ vibrationally excited molecular species can slow down the process. ${ }^{62,83,84}$ The latter effect is caused by superelastic collisions between electrons and (mostly) vibrationally excited molecules; the vibrational temperature of the nitrogen can be an order of magnitude larger than the rotational temperature under similar operating conditions. ${ }^{9,21,83}$

The time required for nitrogen to attain these high vibrational states is on the order of $10^{-5}-10^{-3}$ seconds $^{83,84}$ so the electron-temperature decrease in the afterglow will be highly dependant on the pulse width—operating the PGD in the microsecond regime may not display the superelastic-collison effect. The internal energy contained in the various modes of the nitrogen molecule can increase the time the electrons take to reach 
the low temperatures required for efficient recombination. ${ }^{85}$ At high nitrogen concentrations (time zero in Figure 4.12), superelastic collisions almost completely prevent the electrons from thermalizing before they are lost by ambipolar diffusion ${ }^{30}$ to the chamber walls. Under these conditions, recombination effects are not observed. As the quantity of nitrogen decreases—with increasing time after the valve closure- the possibility for superelastic collisions decreases, so the time-delay before recombination occurs also decreases.

The effects of $1 \%$ nitrogen on the afterglow process are shown to be multifaceted. At $1 \%$ nitrogen, charge transfer with argon ions-and subsequent dissociative recombination of the resultant nitrogen ions - decreases the number density of argon ions by an order of magnitude. High-energy vibrational modes, developed during the $5 \mathrm{~ms}$ before the voltage termination, prevent electrons from kinetically cooling in the afterglow. Consequently, very little recombination occurs for argon ions and the afterglow emissions are vastly reduced. As the nitrogen concentration decreases, fewer argon ions are removed by charge transfer reactions, fewer superelestic collisions are possible for electrons, and more CRC occurs for argon in the afterglow.

\subsubsection{Effect of $\mathrm{N}_{2}$ on analyte signals}

Whereas the excitation of sputtered analyte atoms in GDs and PGDs occurs almost exclusively by electron excitation (see Chapter 3), ionization occurs by three predominant processes. ${ }^{30,33,34,36}$ These processes are Penning Ionization (PI), ${ }^{86}$ charge transfer (CT), and electron ionization, given, respectively, by the three equations below.

$$
\operatorname{Ar}^{\mathrm{m}}\left({ }^{3} \mathrm{P}_{2} /{ }^{3} \mathrm{P}_{0}\right)+\mathrm{M}^{0} \rightarrow \mathrm{Ar}^{0}+\mathrm{M}^{\mathrm{n}+}+\mathrm{ne}^{-}
$$




$$
\begin{aligned}
& \operatorname{Ar}^{+}\left({ }^{2} \mathrm{P}_{1 / 2} /{ }^{2} \mathrm{P}_{3 / 2}\right)+\mathrm{M}^{0} \rightarrow \mathrm{Ar}^{0}+\mathrm{M}^{\mathrm{n}+^{*}}+\mathrm{ne}^{-} \\
& \mathrm{e}^{-}+\mathrm{M}^{0} \rightarrow \mathrm{M}^{\mathrm{n}+}+\mathrm{ne}^{-}
\end{aligned}
$$

$\mathrm{Ar}^{\mathrm{m}}$ refers to one of the metastable states of argon (at 11.55 and $\left.11.72 \mathrm{eV}\right), \mathrm{M}^{0}$ is the ground state sputtered metal atom, and $\mathrm{Ar}^{0}$ is the ground state of $\operatorname{argon}$, and $\mathrm{M}^{\mathrm{n}+*}$ is a ground- or excited ion state of the metal. If the ionization potential (IP) of $\mathrm{M}$ is less than the metastable state of argon (as is the case for all metals in the periodic table), then PI will occur at approximately $1 / 5$ the collision frequency. ${ }^{55,87}$ If the second IP of M is also below the metastable states, as is common for the rare earth elements, ${ }^{88} \mathrm{M}$ can be doublyionized in a single PI collision. ${ }^{89,90}$ Several reports have shown that Penning ionization is one of the most dominant ionization mechanisms in unconfined GDs below 2 Torr. ${ }^{27,91}$ When operated at higher pressures, such as in the Grimm source, ${ }^{16,20,56,60,92-98}$ or when confined, such as in hollow cathode geometries, ${ }^{94,98-101}$ excitation and ionization are less dependant on metastable atom populations. At higher pressures, metastable atom number densities are reduced by increases in metastable-metastable collisions, electron deexcitation, and collisional de-excitation by atoms and ions. The optimum pressure range for maximum metastable formation is $\sim 0.8$ Torr, ${ }^{69,102,103}$ as used in these studies.

CT reactions are most efficient when an efficient overlap exists between the argon ion and the neutral with which it reacts. ${ }^{104}$ As discussed in the previous chapter, the ${ }^{2} \mathrm{P}_{1 / 2}$ ground state argon ion overlaps well with an excited state of the copper ion, resulting in enhanced population of this state with respect to states close in energy. This enhancement of the $224.7 \mathrm{~nm}$ line for $\mathrm{Cu}$ II, indicating CT, has been witnessed mostly in higher pressure (>1 Torr) discharges, ${ }^{15,18,94,95,105}$ and less so in lower pressure GDs. ${ }^{103}$ 
Electron excitation and ionization are more dominant in higher-pressure GDs when PI contributions are reduced. Electron temperatures and excitation temperatures have been determined in a number of different steady state glow discharge sources using a number of different methods. Langmuir probe measurements ${ }^{91}$ and spectroscopic studies $^{1,9,21,60,106-108}$ show that the electron temperature is not particularly sensitive to operating conditions, but is sensitive to the sample cathode. ${ }^{91}$

Of these three mechanisms, Penning ionization is expected to dominate ionization processes, while electron excitation from the ground state is expected to dominate the excitation processes for the sputtered atoms. Charge transfer, while possible for iron, ${ }^{109}$ is not expected to be important at these pressures.

\subsubsection{Fe atom emissions}

Figure 4.13 shows the effect of $1 \%$ nitrogen addition on the emission signals of Fe I. The two lines observed are the $3 \mathrm{~d}^{6} \cdot 4 \mathrm{~s}^{2}-3 \mathrm{~d}^{6} 4 \mathrm{~s} .4 \mathrm{p}(3.33-0.00 \mathrm{eV})$ transition at 371.99 $\mathrm{nm}$, and the $3 \mathrm{~d}^{7} 4 \mathrm{~s}-3 \mathrm{~d}^{7} 4 \mathrm{p}(4.41-0.99 \mathrm{eV})$ transition at $361.88 \mathrm{~nm}$. The heavy lines are the emissions collected under normal operating conditions (no nitrogen added) and behave similarly to the copper lines discussed elsewhere. ${ }^{110,111}$ Close to the cathode $(<2 \mathrm{~mm})$, very little afterpeak is observed, and further from the cathode $(>3 \mathrm{~mm})$ the afterpeak intensity increases, relative to the steady-state signals. As discussed in the previous chapter, the CRC process that occurs when the voltage is removed accounts for the presence of the afterpeak. Figure 4.13 shows that when $1 \%$ nitrogen is added the afterpeak emissions are no longer prevalent. This follows the same trend observed for argon atom emissions described above. With $1 \%$ nitrogen added, high-lying vibrational 
states—excited during voltage-on period-provide kinetic energy to electrons in the afterglow via superelastic collisions. The electrons, therefore, take longer to thermalize and consequently some will be lost by diffusion to the walls; thus the CRC is reduced.

The reduction in emission intensity during the steady state could be caused by a reduction in electron temperature or number density. The nitrogen absorbs energy into internal modes during the voltage-on period, ${ }^{62,84}$ and this energy transfer process presumably reduces the electrons' kinetic energy. In the absence of electron-temperature measurements, such speculation cannot be validated. Wagatsuma and coworkers have shown that reduced emission intensities in $\mathrm{Ar} / \mathrm{N}_{2}$ plasmas could be caused, in part, by a reduction in sputtering rate. ${ }^{15}$ A lower sputtering rate, and hence lower atom number density in the plasma would cause a uniform reduction in emission intensity. Figure 4.13 shows that the reduction in emission intensity in the steady-state is indeed fairly uniform in space with approximately 50\% reduction. This is shown more clearly in Figure 4.14, where the steady-state emission at $371.99 \mathrm{~nm}$ is plotted as a function of distance. The same effect is not as conspicuous for the bulk plasma gas (argon) as seen by the emission intensity at $811.5 \mathrm{~nm}$ in Figure 4.15. The emissions are not reduced to the same extent as the iron lines, indicating the specificity of emission reduction. 

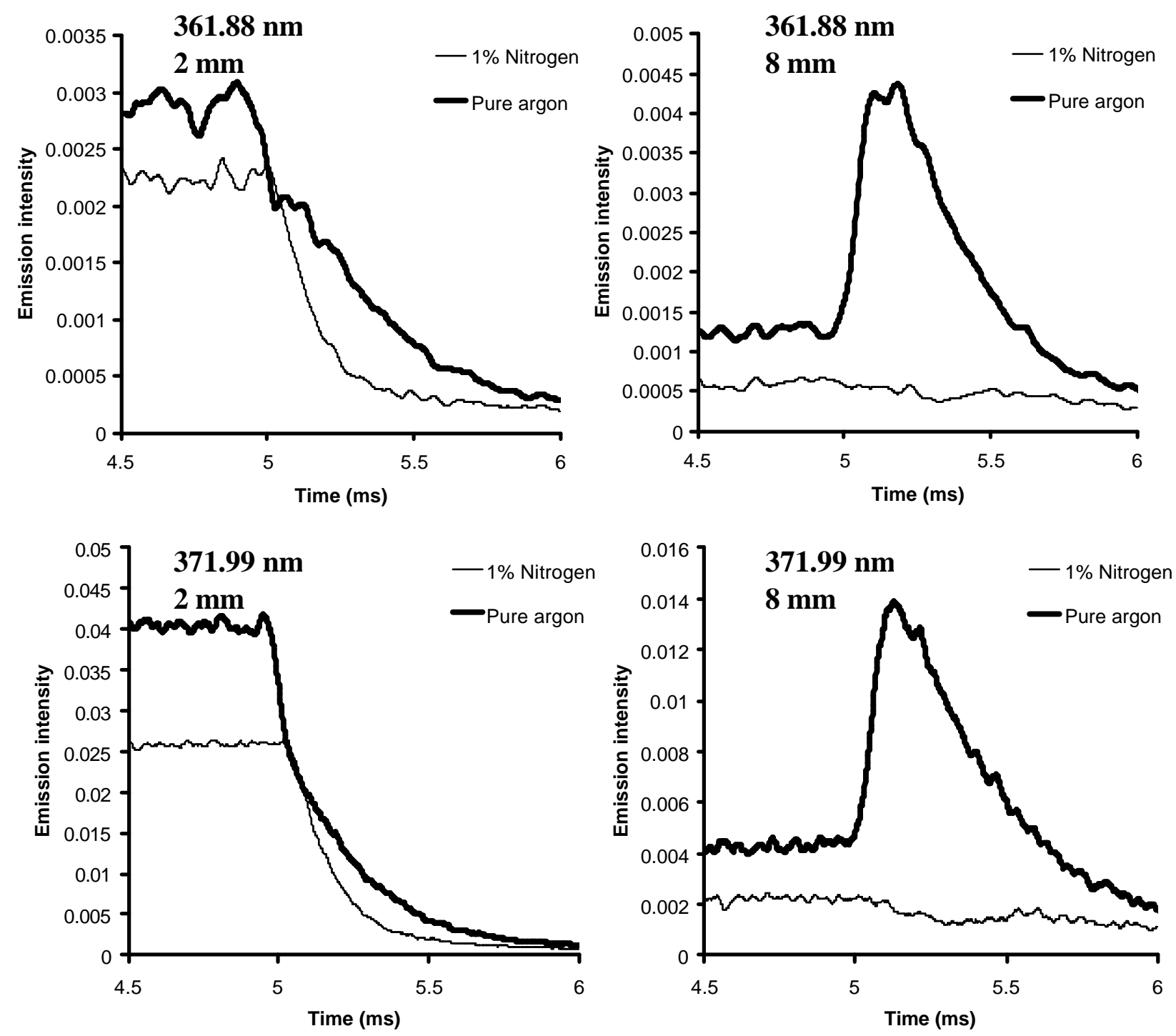

Fig. 4.13. Effect of $1 \%$ nitrogen on sputtered atom emissions. Each plot is labeled according to the emission wavelength and sampling height above the cathode. 5.0 ms pulse length, 25\% duty cycle, 0.8 Torr Ar, $1.5 \mathrm{~W}$ peak power, Fe cathode. 


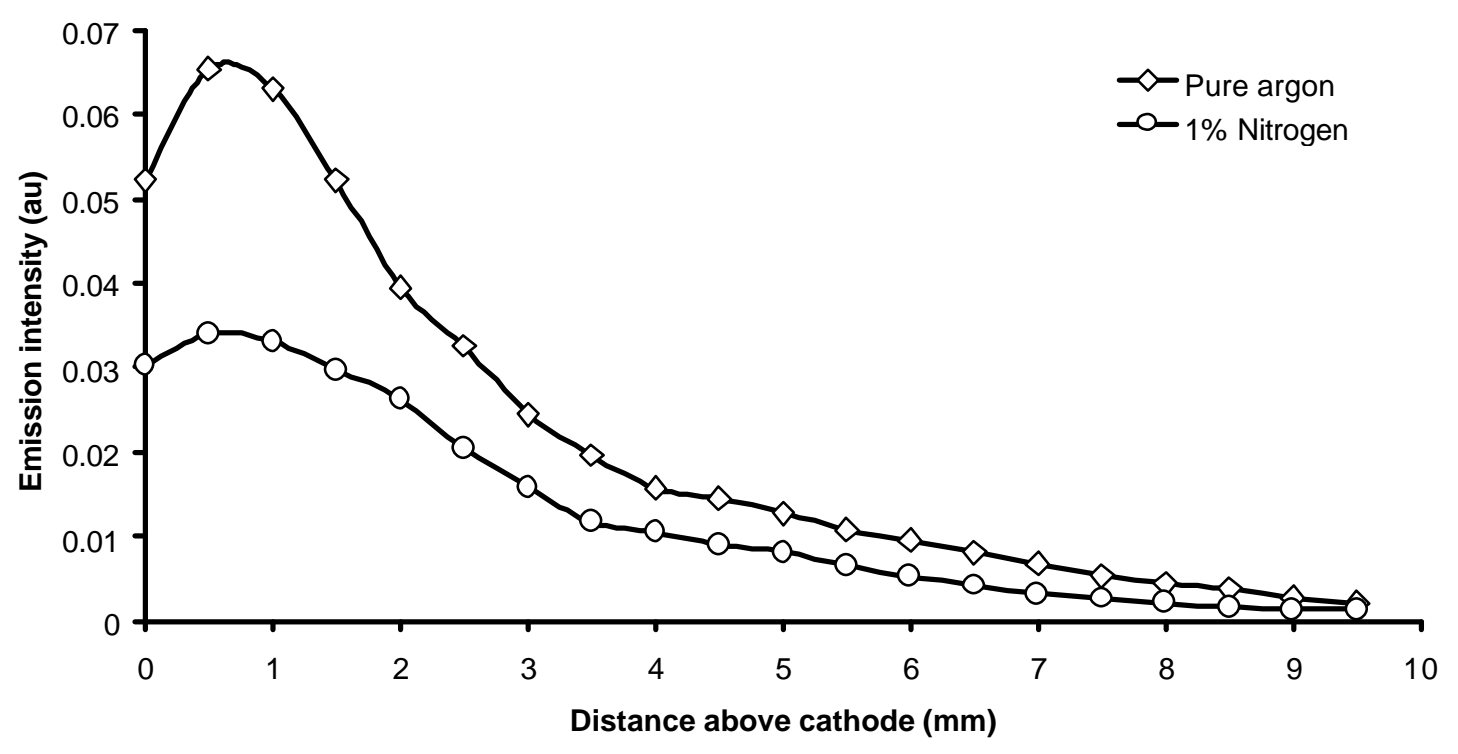

Fig. 4.14. Emission intensity at $4.9 \mathrm{~ms}$ at $371.99 \mathrm{~nm}$ as a function of distance above the cathode to show the uniform reduction in signal with $1 \% \mathrm{~N}_{2}$ added. Conditions given in Fig. 4.13.

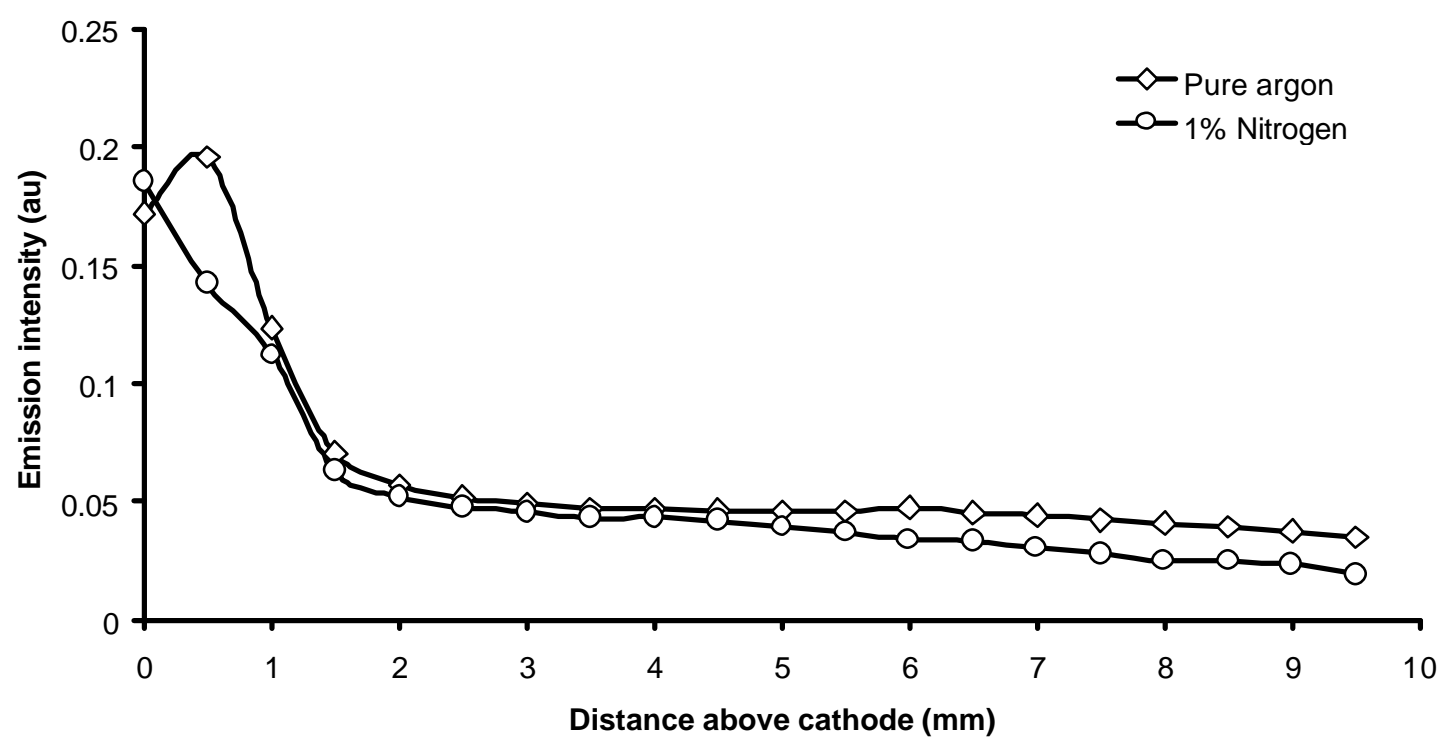

Fig. 4.15. Emission intensity at $4.9 \mathrm{~ms}$ at $811.5 \mathrm{~nm}$ as a function of distance above the cathode to show the (lack of) effect of $1 \% \mathrm{~N}_{2}$ addition. Conditions given in Fig. 4.13 . 
Fang and Marcus report that reduced sputter yields are expected when lighter atoms are used as projectiles for cathodic sputtering. ${ }^{15}$ The occurrence of $\mathrm{N}_{2}^{+}$or $\mathrm{N}^{+}$ charge carriers impinging on the cathode surface could produce the lower sputtering yields. Wagatsuma and Hirokawa note with the introduction of relatively small amounts of nitrogen into argon GDs $(<1 \%)$, the emission intensity is a good predictor of the sputtering rate (as determined gravimetrically). The 40-50\% reduction in emission intensity observed here indicates a $40-50 \%$ decrease in sputter rate.

Before progressing, it should be noted that one of the desirable features of PGDs for chemical analysis is the ability to provide a reservoir of sputtered atoms for atomic

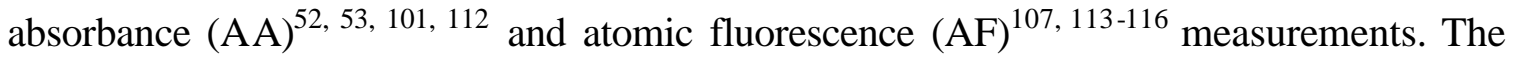
advantages of PGDs for these analyses is that ground state atoms are produced in the gas phase in relatively high yields, with low kinetic energies, and with little background emission. The latter benefit is realized by performing the AA or AF analysis after pulse termination, where plasma emissions are reduced. As we have seen, plasma emissions actually increase for a short time in the afterglow, so measurements would most-benefit if made after all the plasma emissions have decayed. However, during this time the sputtered atoms slowly diffuse to the cathode walls ${ }^{52,107}$ so their number density decreases. A compromise must be established between making the $\mathrm{AA}$ or $\mathrm{AF}$ measurement late enough to avoid interfering emissions from the afterglow and making the measurements soon enough to benefit from the highest number densities of sputtered atoms. The addition of small quantities of nitrogen has been shown here to be particularly effective at preventing the afterglow emissions; thereby, allowing $\mathrm{AA}$ or $\mathrm{AF}$ 
measurements to be made sooner after pulse termination. Increasing the GD voltage could presumably make up for the small reduction in sputtering rate.

\subsubsection{Fe ion signals}

Figure 4.16 shows the ${ }^{56} \mathrm{Fe}^{+}$signal as a function of sampling distance above the cathode taken at $4.9 \mathrm{~ms}$ after pulse initiation. These signals exemplify steady-state signals. The addition of $1 \%$ nitrogen drastically reduces the iron signals but the effect is not uniform across the discharge (notice the logarithmic scale). Close to the cathode (2-4 $\mathrm{mm}$ ), iron ion signals are reduced by factors of 10-20, but at greater sampling distances (5-7 mm), iron ion signals decreased by up to a factor of 30 . The difference between emission attenuation factor (2 throughout) and ion attenuation factor (10-30) for $\mathrm{Fe}$ is too great to be due to an experimental artifact. Clearly, excitation and ionization processes are strongly decoupled, especially in the region above $4 \mathrm{~mm}$.

This decoupling was also observed by Ratliff and Harrison, ${ }^{14}$ by the timedependent emission and ion signal monitoring of $\mathrm{Cu}$ in response to pulses of water vapor introduced into the GD. Cu emissions were only briefly reduced by the introduction of a pulse of $\mathrm{H}_{\mathbf{O}} \mathrm{O}$, but $\mathrm{Cu}$ ion signals were reduced for a considerably longer time. This indicates that although the electron-energy-distribution-function (EEDF) was only slightly affected by the pulse of $\mathrm{H}_{2} \mathrm{O}$, the mechanism responsible for ionizing the copper was more sensitive to the trace amounts of water. This decoupling demonstrates the minor role that electrons play in ionizing sputtered atoms under these conditions, and the major role that the metastables play. 


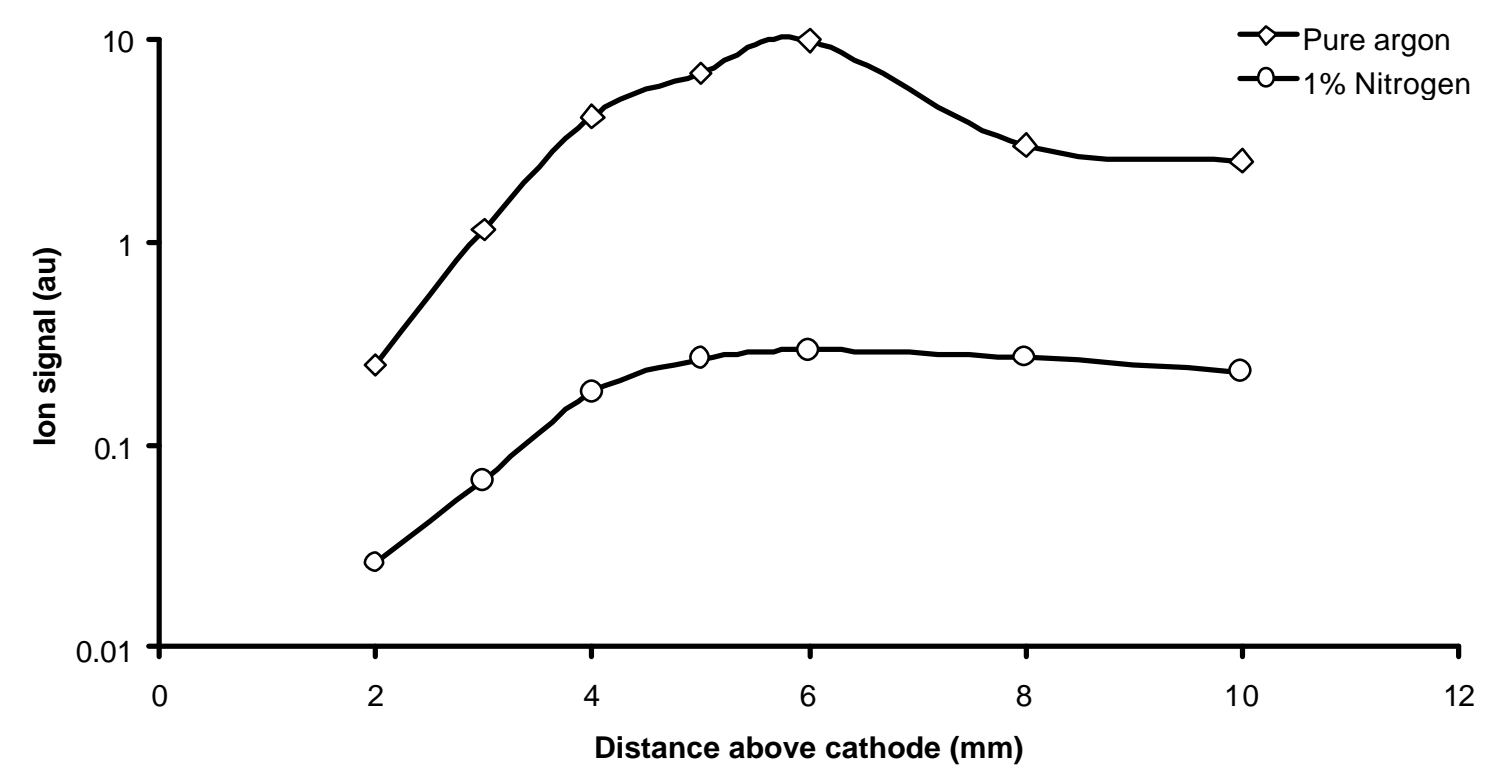

Fig. 4.16. ${ }^{56} \mathrm{Fe}^{+}$ion signals as a function of sampling height above the cathode to show the effect of $1 \%$ nitrogen on the ionization of sputtered metal atoms. Ion signals measured at $4.9 \mathrm{~ms}$, representing steady-state conditions. $5.0 \mathrm{~ms}$ pulse length, $25 \%$ duty cycle, 0.8 Torr Ar, $\sim 1.5 \mathrm{~W}$ peak power, Fe cathode.

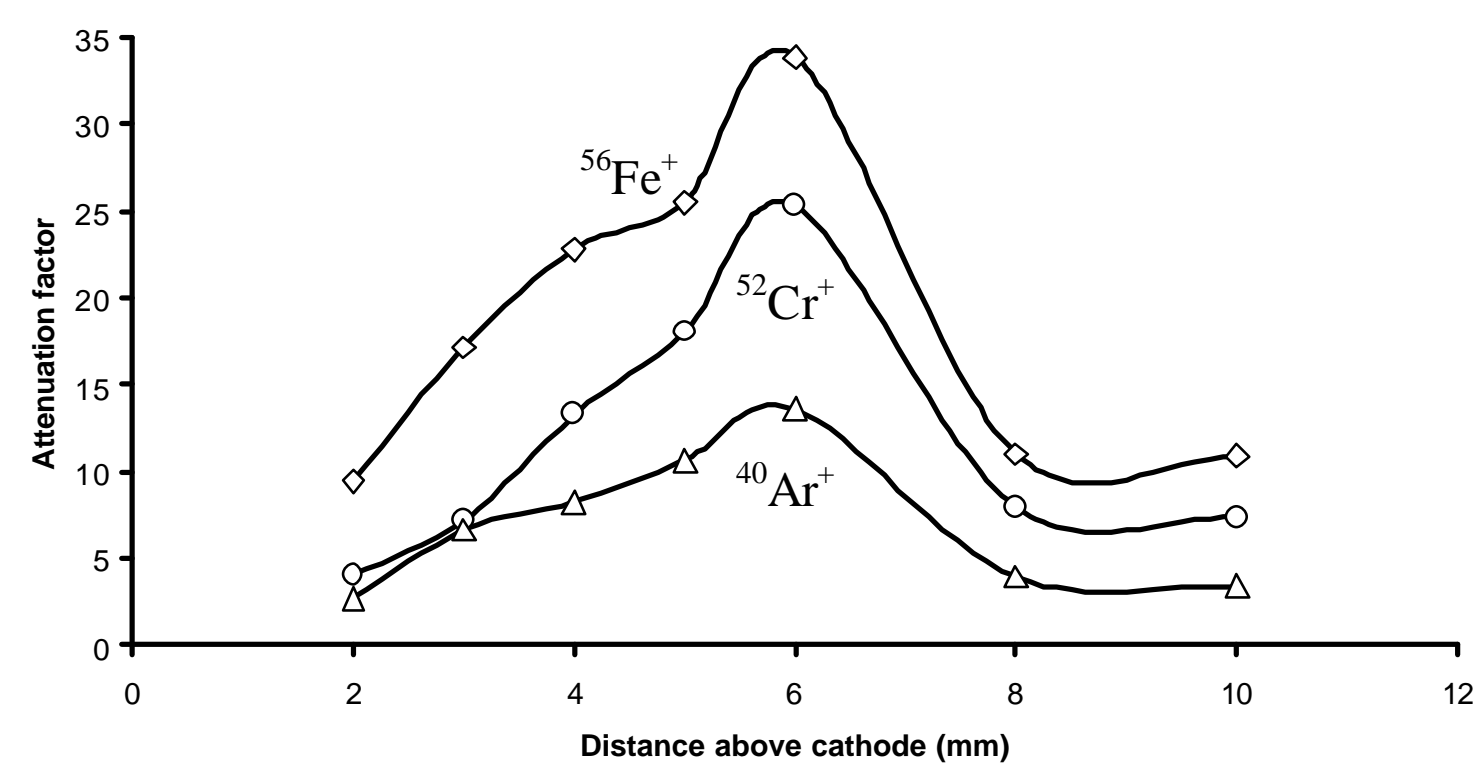

Fig. 4.17. Attenuation factors for the steady-state signals of different ions in response to the addition of $1 \%$ nitrogen. Conditions given above. 
The addition of $1 \%$ nitrogen to the plasma had different effects on different ions in the spectra. All the sputtered analyte ion signals responded in a similar way, with attenuation factors in the range 5-30 (depending on distance) as demonstrated for ${ }^{56} \mathrm{Fe}^{+}$ and ${ }^{52} \mathrm{Cr}^{+}$in Figure 4.17. The addition of nitrogen also reduced the number of $\mathrm{Ar}^{+}$ions by factors of 5-13 (as expected by charge transfer collisions) and $\mathrm{Ar}_{2}{ }^{+}$ions by factors of 213, depending on distance, with the largest attenuation occurring at $\sim 4-6 \mathrm{~mm}$. The $\mathrm{ArH}^{+}$, $\mathrm{H}_{3}{ }^{+}$and $\mathrm{Ar}^{2+}$ signals were considerably more attenuated by the addition of nitrogen than all the other ions in the spectra, as seen in Figure 4.18.

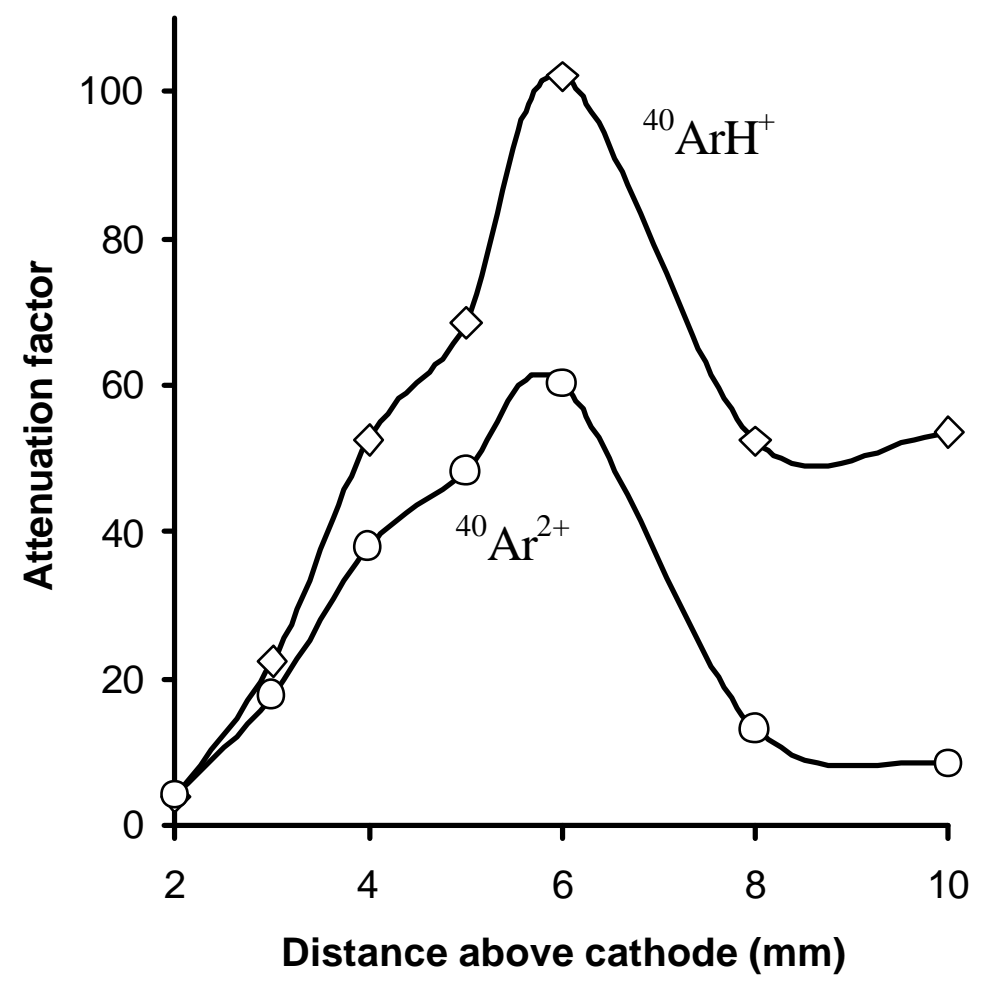

Fig. 4.18. Attenuation factors for the steady-state signals of $\mathrm{Ar}^{2+}$ and $\mathrm{ArH}^{+}$in response to the addition of $1 \%$ nitrogen. Conditions given in Figure 4.16. 
The added nitrogen completely eliminated the $\mathrm{H}_{3}{ }^{+}$signal. $\mathrm{ArH}^{+}$and $\mathrm{Ar}^{2+}$ signals were reduced by factors of 10-100, as shown in Figure 4.18. These ions are obviously much more sensitive to the $\mathrm{N}_{2}$ addition than $\mathrm{Ar}^{+}, \mathrm{Ar}_{2}{ }^{+}$, or the other analyte ions, indicating, perhaps, that the three ions have related precursors. This behavior would also be consistent if the three ions in question required two excited- or ionized argon atoms as precursors, and all the other attenuated ions only relied on one excited/ionized precursor. Under these circumstances, a decrease in the excited/ion states of argon would decrease the $\mathrm{H}_{3}{ }^{+}, \mathrm{ArH}^{+}$, and $\mathrm{Ar}^{2+}$ ions to the square of the loss of the excited/ion states, but would reduce the other ions proportionally to the loss of excited/ion states. Another possibility is that the added nitrogen introduces additional destruction mechanisms for these ions. If CT between $\mathrm{Ar}^{2+}$ and $\mathrm{N}_{2}$ is a quantum-allowed process and occurred at the collision frequency, the rate for this reaction would be approximately twice as fast as the CT reaction between $\mathrm{Ar}^{+}$and $\mathrm{N}_{2} \cdot{ }^{117}$ Based on this premise, the added nitrogen would reduce $\mathrm{Ar}^{2+}$ signal by factors of $10-26$, depending on height above the cathode. That the attenuation factor is twice this large again indicates that the nitrogen must also be reducing the formation of $\mathrm{Ar}^{2+}$.

The most probable population mechanism for populating the $27.63 \mathrm{eV}$ ground state of $\mathrm{Ar}^{2+}$ is electron excitation.

$$
\mathrm{Ar}^{0}+\mathrm{e}_{\text {fast }}^{-} \rightarrow \mathrm{Ar}^{2+}+3 \mathrm{e}_{\text {slow }}^{-}
$$

The vast reduction in the $\mathrm{Ar}^{2+}$ ions would require that the high-energy tail of the EEDF be drastically reduced by the addition of $1 \%$ nitrogen. Indeed, the ability for nitrogen to undergo inelastic collisions with electrons and to absorb the electron's kinetic energy is well known. ${ }^{118-120}$ Even at concentrations as low as $1 \%$ in argon, nitrogen greatly reduces 
the average energy of the EEDF. Two other energetically feasible reactions for the formation of doubly charged argon ions are given by the equations,

$$
\begin{aligned}
& \mathrm{Ar}^{* *}+\mathrm{Ar}^{* *} \rightarrow \mathrm{Ar}^{2+}+\mathrm{Ar}^{0}+2 \mathrm{e}^{-} \\
& \mathrm{Ar}^{* *}+\mathrm{Ar}^{+} \rightarrow \mathrm{Ar}^{2+}+\mathrm{Ar}^{0}+\mathrm{e}^{-}
\end{aligned}
$$

where $\mathrm{Ar}^{* *}$ is an excited state of argon above $13.81 \mathrm{eV}$ (4.8) or $11.9 \mathrm{eV}$ (4.9), respectively. No reference to these reactions, or their quantum allowedness, could be found in the extant literature. Reactions (4.8) and (4.9) both rely on two highly energetic states of argon to produce the doubly charged ion, and would consequently be sensitive to the square of a reduction in $\mathrm{Ar}^{* *} / \mathrm{Ar}^{+}$, as required.

Possible reactions leading to the formation of $\mathrm{ArH}^{+}$are ${ }^{12,41,121}$

$$
\begin{aligned}
& \mathrm{Ar}^{* *}+\mathrm{H}_{2} \mathrm{O} \rightarrow \mathrm{ArH}^{+}+\mathrm{OH}^{-} \\
& \mathrm{Ar}^{* *}+\mathrm{H}_{2} \rightarrow \mathrm{ArH}^{+}+\mathrm{H}^{-} \\
& \mathrm{Ar}^{* *}+\mathrm{H} \rightarrow \mathrm{ArH}^{+}+\mathrm{e}^{-}
\end{aligned}
$$

In order to be thermo-neutral, reactions (4.8) and (4.9) require total internal energies of 13.1 and $13.5 \mathrm{eV}$, respectively (neglecting kinetic energy effects). ${ }^{88,}{ }^{122}$ This would require an energy level above the $4 \mathrm{~s}$ metastable states of argon. Reaction (4.12) can proceed if the excited state is one of the metastable states at 11.55 or $11.72 \mathrm{eV}$. The formation of $\mathrm{H}$ in reaction (4.12) can occur by a variety of reactions, including ${ }^{121}$

$$
\begin{aligned}
& \mathrm{Ar}^{\mathrm{m}}+\mathrm{H}_{2} \mathrm{O} \rightarrow \mathrm{Ar}^{0}+\mathrm{OH}+\mathrm{H} \\
& \mathrm{Ar}^{+}+\mathrm{H}_{2} \rightarrow \mathrm{ArH}^{+}+\mathrm{H}
\end{aligned}
$$

both of which are exothermic as written and require a highly-energetic precursor.

The dominant formation mechanism for $\mathrm{H}_{3}{ }^{+}$is expected to be $e^{121}$

$$
\mathrm{ArH}^{+}+\mathrm{H}_{2} \rightarrow \mathrm{Ar}^{0}+\mathrm{H}_{3}^{+}
$$


which is exothermic as written. The formation of $\mathrm{H}_{3}{ }^{+}$is therefore dependant on the $\mathrm{ArH}^{+}$ precursor. The vast reduction in $\mathrm{ArH}^{+}$by the addition of $1 \%$ nitrogen is presumably the cause for the elimination of $\mathrm{H}_{3}{ }^{+}$. The added nitrogen is also able to prevent/decrease the formation of $\mathrm{H}_{3}{ }^{+}$by the reactions

$$
\begin{aligned}
& \mathrm{ArH}^{+}+\mathrm{N}_{2} \rightarrow \mathrm{Ar}^{0}+\mathrm{HN}_{2}^{+} \\
& \mathrm{H}_{3}^{+}+\mathrm{N}_{2} \rightarrow \mathrm{H}_{2}+\mathrm{HN}_{2}^{+}
\end{aligned}
$$

which are presumably exothermic based on the appearance of large quantities of $\mathrm{HN}_{2}{ }^{+}$in the spectra with nitrogen added. Reaction (4.16) should proceed at a rate similar to that for the $\mathrm{CT}$ reaction between $\mathrm{Ar}^{+}$and $\mathrm{Fe}^{0}$, so the additional destruction channel for $\mathrm{ArH}^{+}$ can only account for a $\sim 5-13$ fold reduction in signal. Again, this suggests that nitrogen is preventing the formation of $\mathrm{ArH}^{+}$, as well as providing additional destruction channels.

The argon ions can be formed directly by electron ionization, or by electron ionization from the metastable states.

$$
\begin{aligned}
& \mathrm{Ar}^{0}+\mathrm{e}_{\text {fast }} \rightarrow \mathrm{Ar}^{+}+2 \mathrm{e}_{\text {slow }}^{-} \\
& \mathrm{Ar}^{\mathrm{m}}+\mathrm{e}_{\text {fast }}^{-} \rightarrow \mathrm{Ar}^{+}+2 \mathrm{e}_{\text {slow }}^{-}
\end{aligned}
$$

The reduction of argon ions observed in Figure 4.17 might be related to additional destruction mechanisms created by the added nitrogen, rather than inhibiting a production process. One such additional loss process induced by $\mathrm{N}_{2}$ is the charge transfer reaction given in reaction (4.2). The reduction in the metal ion signals in the steady-state regime is undoubtedly related to the reductions in sputter yield and metastable states. The fact that the sputtering rate decreased by a factor of $\sim 2$, but the ionization efficiency decreased by a factor of 5-30, is testimony to the importance of PI in ionizing the sputtered atoms. 
The preceding pages have shown that there are several mechanisms to explain the extreme attenuation rates of $\mathrm{H}_{3}{ }^{+}, \mathrm{ArH}^{+}$, and $\mathrm{Ar}^{2+}$ compared to the other ion signals. The formation of these ions requires either very high-energy electrons $(>27 \mathrm{eV})$, or at least two highly excited or ionized states of argon as precursors. Nitrogen is known to reduce the kinetic energy of electrons when added at $1 \%$ to argon plasmas, and is shown to reduce both the metastable states and the ion states of argon. The other ions are less affected by the addition of $\mathrm{N}_{2}$ (metal ions, $\mathrm{Ar}^{+}$and $\mathrm{Ar}_{2}{ }^{+}$) because they rely on lower energy electrons and/or one excited state of argon. Consideration of additional loss channels created by the addition of nitrogen will contribute to the reduction in the discussed ion signals, but alone cannot account for the attenuation factors observed.

Ions that increased or appeared in the steady-state signals $(4.9 \mathrm{~ms})$ with the addition of $1 \%$ nitrogen included $\mathrm{N}^{+}, \mathrm{O}^{+}, \mathrm{OH}^{+}, \mathrm{H}_{2} \mathrm{O}^{+}, \mathrm{H}_{3} \mathrm{O}^{+}, \mathrm{N}_{2}{ }^{+}, \mathrm{N}_{3}^{+}$, and $\mathrm{N}_{2} \mathrm{H}^{+}$. All these charge carriers are significantly lighter than argon and would reduce the sputter yield, if involved in the sputtering process.

The added nitrogen is shown to have a large effect on the ions signals in the 4-5 ms time regime. At this time, the plasma quite accurately resembles continuous, or steady-state, conditions. Optical experiments show that when the voltage is terminated at the end of the $5 \mathrm{~ms}$ pulse, analyte ions (and argon ions) recombine in a CRC fashion, causing a population inversion in the excited states. One might assume that the observed emissions from the sputtered metal atoms would signify a decrease in the analyte ions in the post-pulse period. That this is not the case has been demonstrated on numerous occasions. ${ }^{49-52,54}$ Figure 4.19 shows a typical response for various ions under normal operating conditions. The argon ion signal is not shown here because its temporal 
characteristics have already been discussed in detail (See discussion on Fig. 4.11). With no nitrogen added, the $\mathrm{Fe}^{+}$and $\mathrm{Cr}^{+}$ion signals decrease immediately following pulse termination, but quickly increase to a maximum around $5.1 \mathrm{~ms}$. The post-pulse maximum is noticeably larger than the steady-state signal. After $5.1 \mathrm{~ms}$ the ion signals decay in a reasonably exponential manner. $\mathrm{ArH}^{+}$displays similar behavior, but the afterpeak maximum is smaller with respect to the steady-state value, and the decay rate is considerably faster. It is assumed that the faster decay rate is due to the faster recombination rate of the diatomic $\mathrm{ArH}^{+}$, as opposed to the monatomic $\mathrm{Fe}^{+}$and $\mathrm{Cr}^{+}$. 

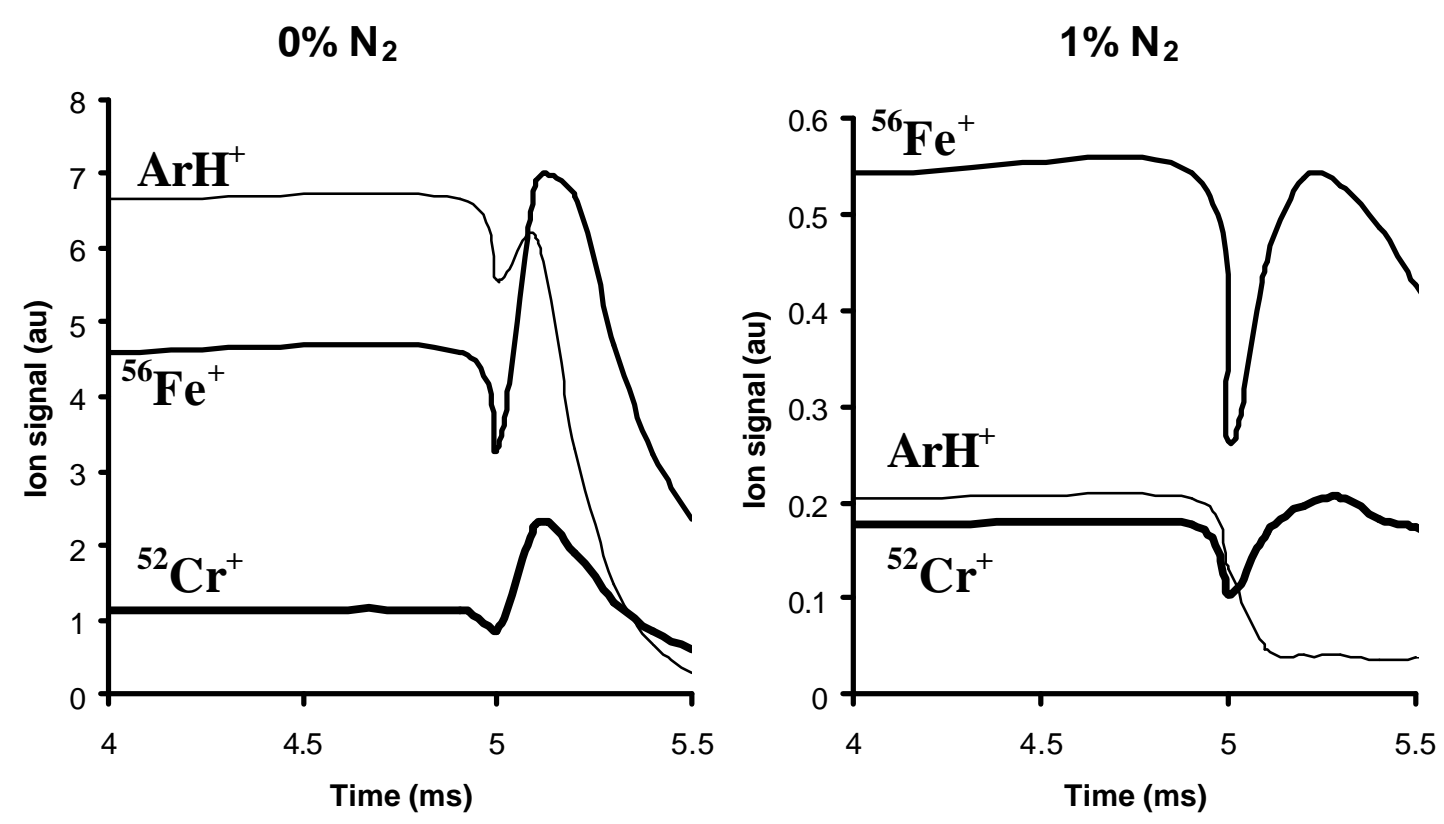

Fig. 4.19. Selected ion signals as a function of time to show the effect of added nitrogen. $5 \mathrm{~mm}$ sampling height, $5.0 \mathrm{~ms}$ pulse length, $25 \%$ duty cycle, 0.8 Torr Ar, $1.5 \mathrm{~W}$ peak power, Fe cathode.

With $1 \%$ nitrogen added, the $\mathrm{ArH}^{+}$ion is severely quenched (as discussed earlier) and no evidence for post-pulse formation is observed. If the $\mathrm{ArH}^{+}$in the afterpeak were formed from $\mathrm{Ar}^{+}$precursors, the afterpeak signal for $\mathrm{ArH}^{+}$should scale with the steadystate signal of $\mathrm{Ar}^{+}$. This coupled with the fact that the $\mathrm{ArH}^{+}$is severely quenched in the afterglow is further evidence for reactions (4.10)-(4.12) above.

After the addition of nitrogen, the afterpeaks are more delayed for $\mathrm{Fe}^{+}$and $\mathrm{Cr}^{+}$, maximizing at $\sim 5.3 \mathrm{~ms}$, and of approximately the same magnitude as the steady-state signals. The afterpeak ion signals are smaller than in pure argon, and are more delayed in time. These findings are entirely consistent with the emission experiments discussed 
earlier in the chapter, viz, with added nitrogen afterpeak emission intensities are reduced in intensity and delayed in time. Although somewhat counter-intuitive, these results demonstrate, unequivocally, that ionization and recombination of the sputtered metal atoms and ions are both enhanced in the afterpeak. These observations are rationalized if the recombination effects follow the ionization effects in time.

Metastable argon atoms, formed during CRC of the afterglow, Penning ionize the sputtered metal atoms and increase the number of these metal ions. The sputtered metal ions also enter into the $\mathrm{CRC}$ behavior, occupying the highest energy levels most abundantly, and decaying radiatively to the ground state. The addition of nitrogen delays the thermalization of the electrons, which in turn delays the recombination of argon ions with electrons. This delays the appearance of metastable states, which delays the appearance of the Penning ionized metal ions.

Consistent with this model are some additional interesting observations. Figures $4.20,4.21$, and 4.22 show the effect of $1 \%$ nitrogen on the afterpeak signals of, respectively, the iron ion (determined by Tof-MS), iron recombination (determined by $\mathrm{AE}$ ), and iron ion (determined by AA). In the pure argon discharge, afterpeak ion signals determined by MS and AA and afterpeak emission signals maximize around 5-6 mm above the cathode. Afterpeak signals in all cases display a steady, but small, decrease closer than $6 \mathrm{~mm}$, and larger decreases at distances greater than $6 \mathrm{~mm}$. The addition of nitrogen prevents PI reaction from occurring and this diminishes the metal ion formation in the afterglow, as observed by AA and ToF-MS. The ions are not available for recombination, so emissions from excited atom states are also diminished. 


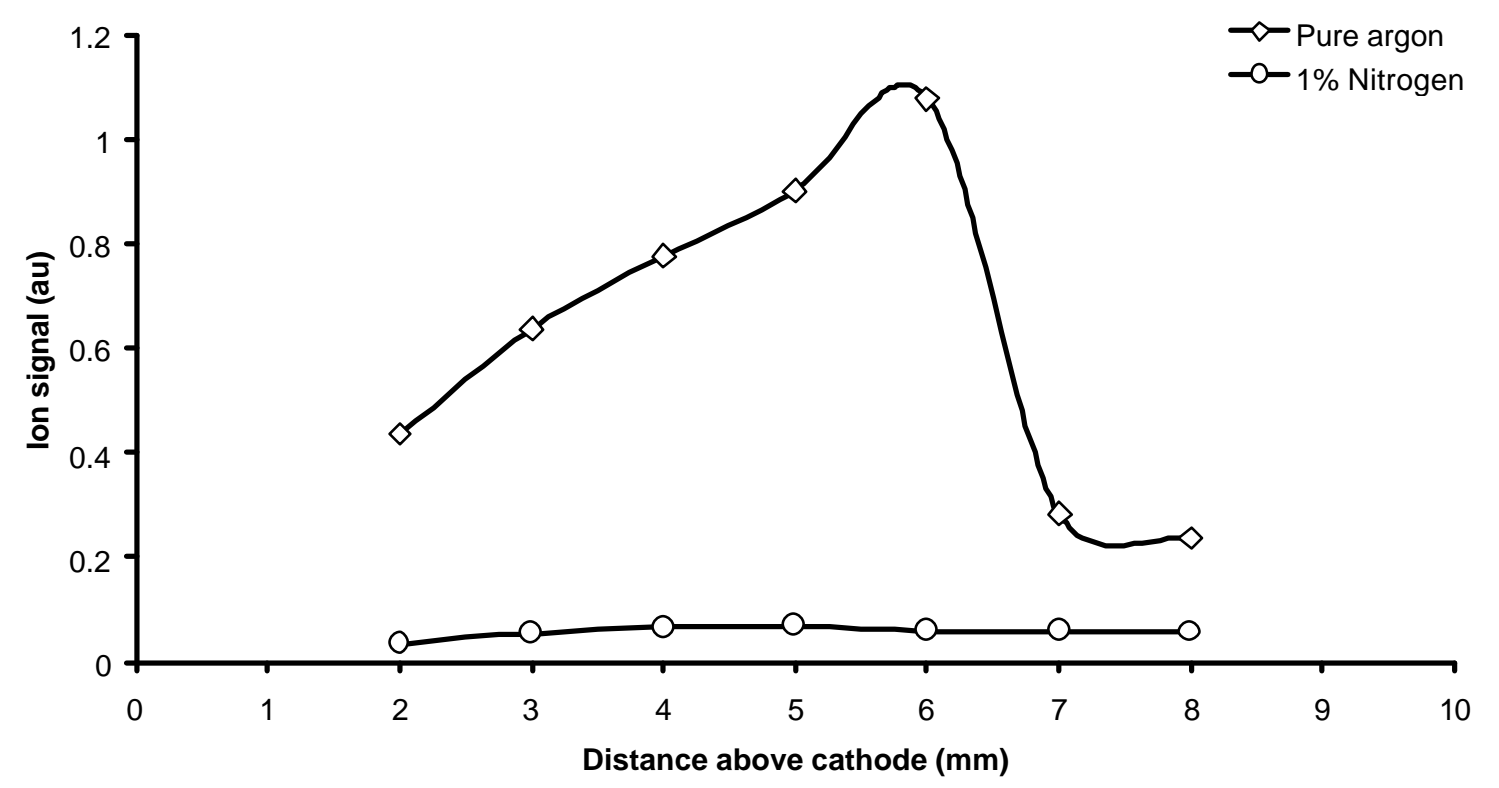

Fig. 4.20. ${ }^{56} \mathrm{Fe}^{+}$signal, determined by ToF-MS, as a function of distance to show the effect of $1 \%$ nitrogen on ionization in the afterglow. $5.0 \mathrm{~ms}$ pulse length, $25 \%$ duty cycle, 0.8 Torr Ar, $1.5 \mathrm{~W}$ peak power, Fe cathode.

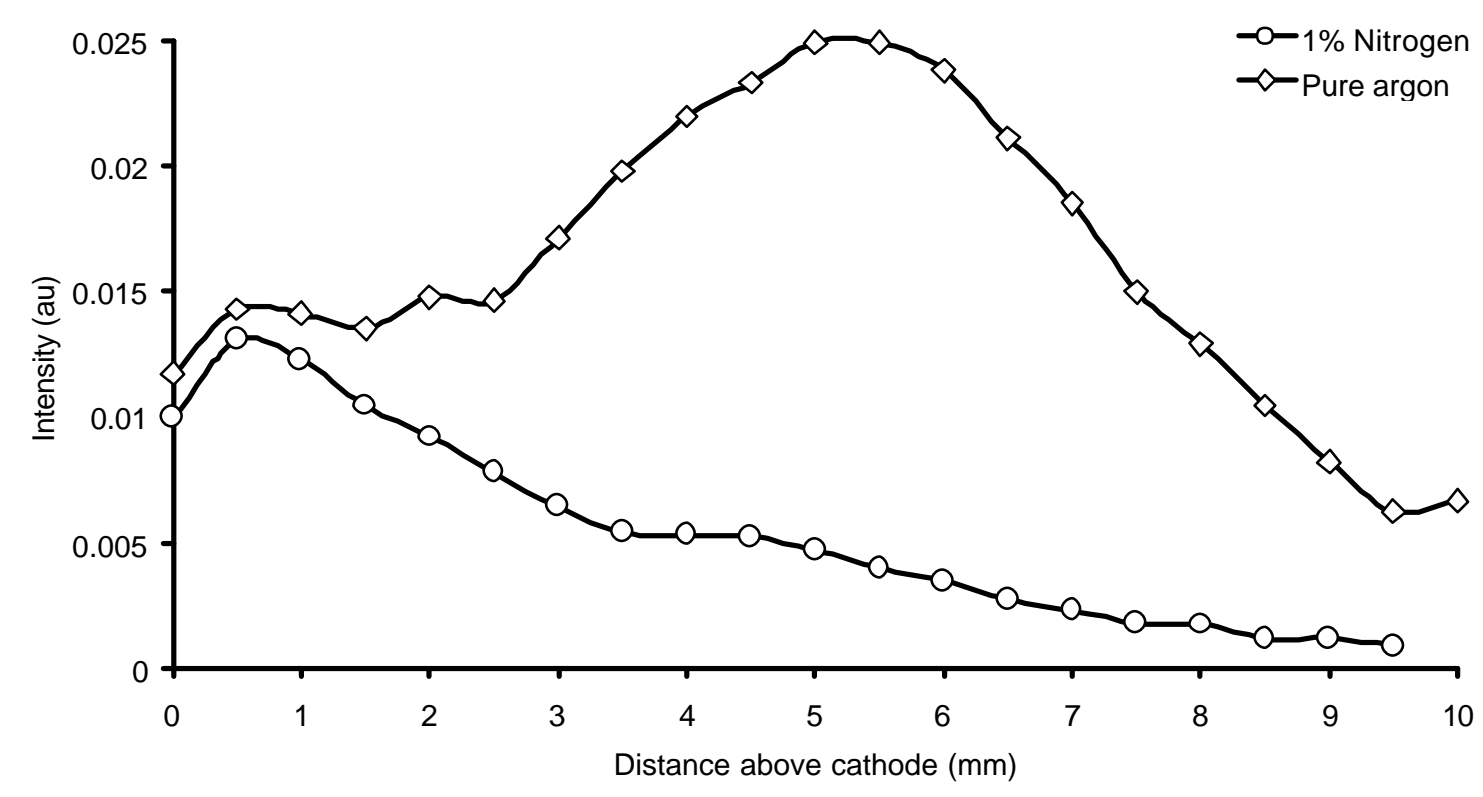

Fig. 4.21. Emission intensity at $371.99 \mathrm{~nm}$ for the Fe I line as a function of distance to show the effect of $1 \%$ nitrogen on the afterglow. Conditions given above. 


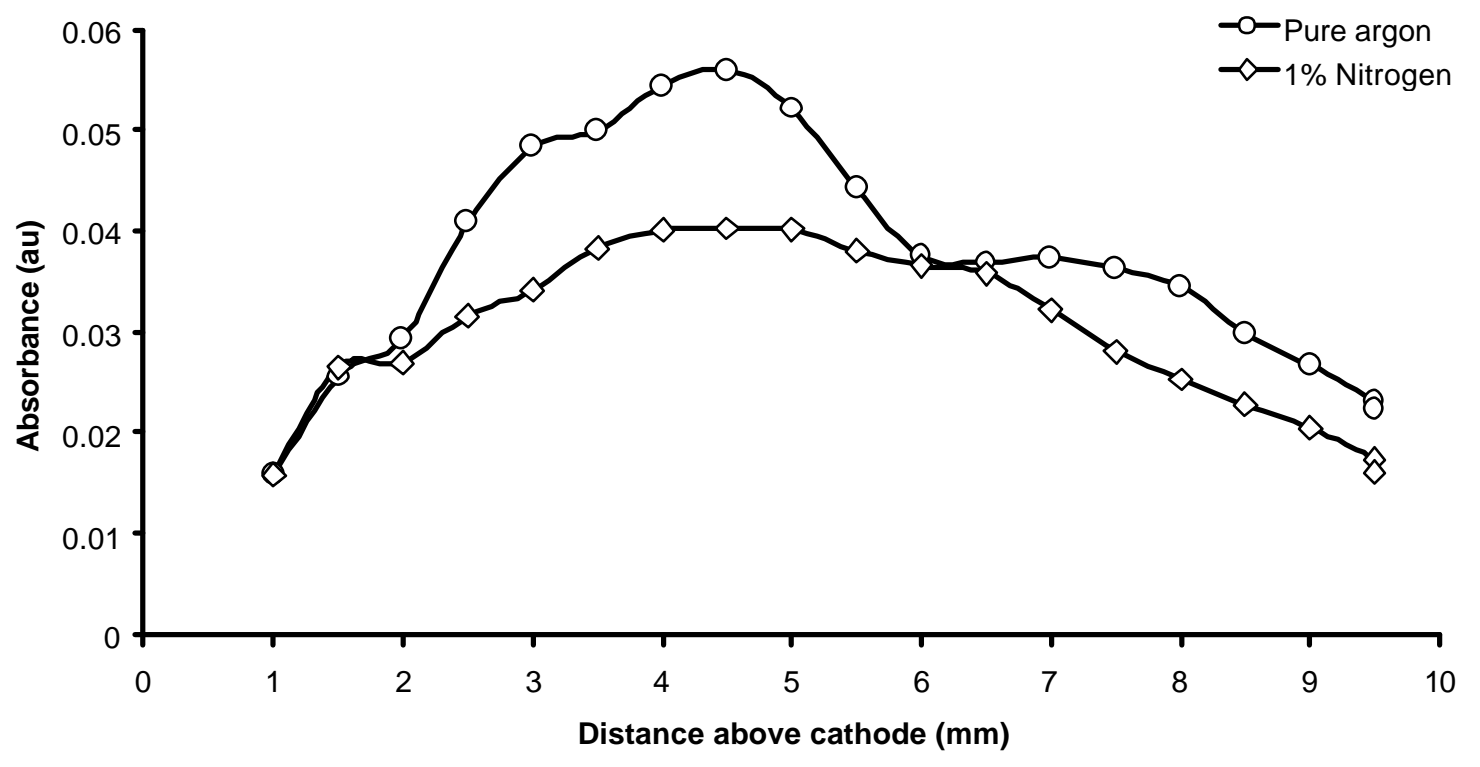

Fig. 4.22. Iron ion absorbance at $259.93 \mathrm{~nm}$ as a function of distance above the cathode to show the effect of $1 \%$ nitrogen addition on the afterglow. Absorption measurements taken at $5.5 \mathrm{~ms}$. 
Due to the prolonged production of metal ions by PI compared to argon ions, one might also anticipate that the iron emissions should also be prolonged. The data illustrated in Figure 4.23 show that this is indeed the case. The afterpeak for the Fe atom emission at $361.88 \mathrm{~nm}$ appears slightly later than the Ar atom emission at $811.5 \mathrm{~nm}$, and maintains this maximum longer than the argon atom emission. While the decay rates are very similar after the afterpeak maximum, the Fe emissions do not reach a base-line level, as do the argon atom emissions. This behavior is not unique to these transitions, but is found for all Ar atom and $\mathrm{Fe}$ atom lines studied. Presuming that the two- or three-body recombination rates are not significantly different for $\mathrm{Ar}^{+}$and $\mathrm{Fe}^{+}$, the prolonged emissions for the Fe atoms must be due to the continual formation of Fe ions by PI.

Using a power supply with a slower fall-time (see experimental section and Chapter 3 for details), similar results were also observed with a copper cathode. Figure 4.24 shows that the emission at the copper atom lines is considerably more prolonged than the emission at the argon atom lines, indicating that the mechanisms discussed above are not unique to iron (indeed, chromium present in the iron cathode also showed similar $\mathrm{AE}$ and MS behavior to iron). The delayed responses of the $\mathrm{Ar}$ I and $\mathrm{Cu}$ I emissions, when the slower fall-time power supply was used, is also consistent with the proposed mechanism in the afterglow. The slower fall time prevents the electrons from cooling as quickly and delays the onset of recombination. Notice, again, how the tailing on the $\mathrm{Cu}$ I emissions do not decay to zero, but are considerably more prolonged than the Ar I emission. 


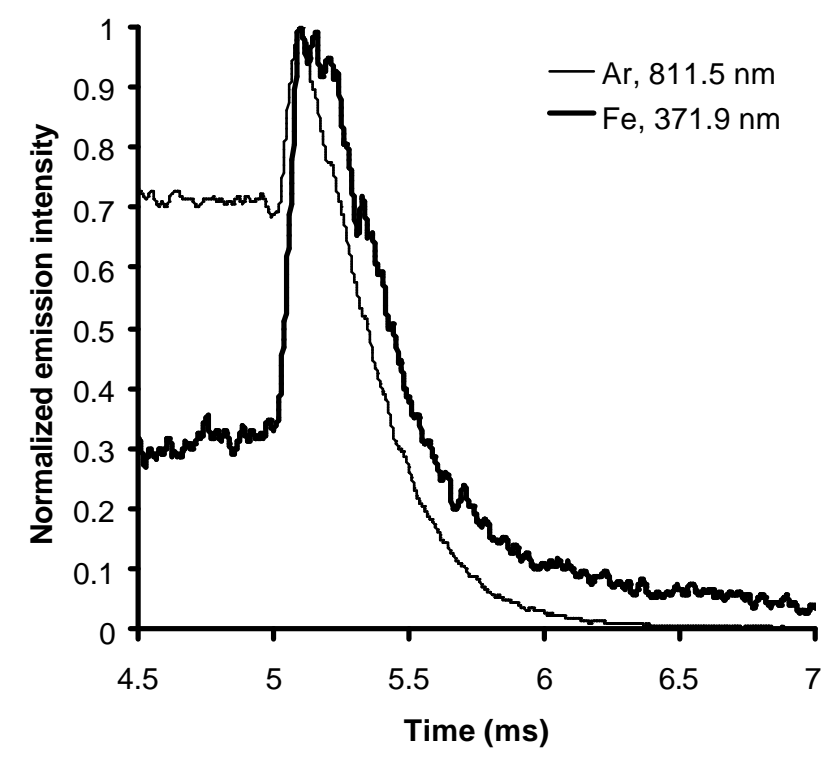

Fig. 4.23. Comparison of the afterpeak emissions for Ar I and Fe I with no nitrogen added. Data collected at $4 \mathrm{~mm}$ above the cathode, $5.0 \mathrm{~ms}$ pulse length, $25 \%$ duty cycle, 0.8 Torr Ar, $1.5 \mathrm{~W}$ peak power, Fe cathode.

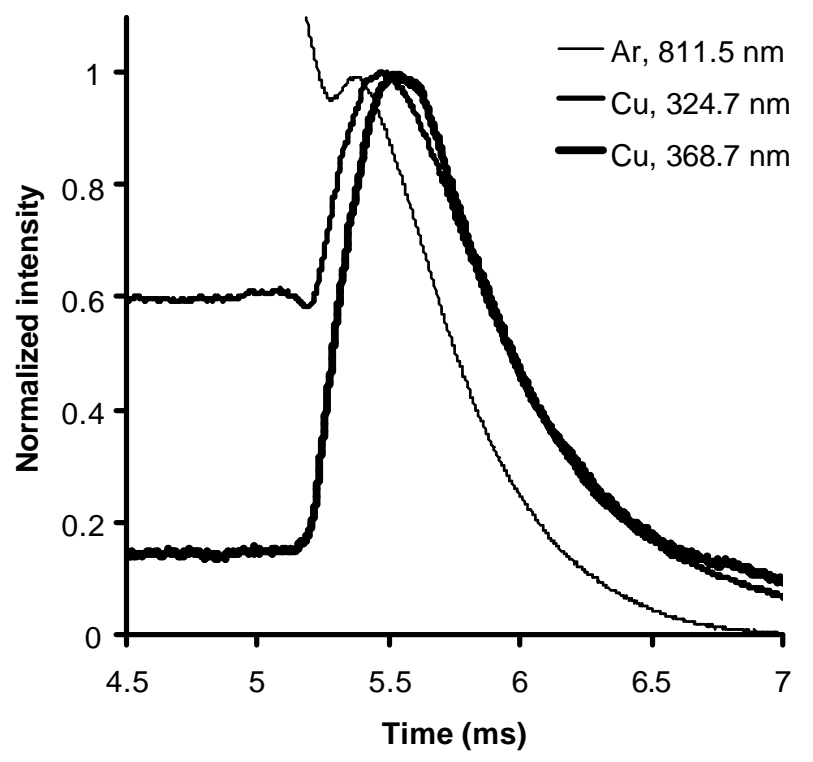

Fig. 4.24. Comparison of the afterpeak emissions for $\mathrm{Ar} \mathrm{I}$ and $\mathrm{Cu} \mathrm{I}$ with no nitrogen added. Data collected at $4 \mathrm{~mm}$ above the cathode, $5.0 \mathrm{~ms}$ pulse length, $25 \%$ duty cycle, 0.8 Torr Ar, $1.5 \mathrm{~W}$ peak power, $\mathrm{Cu}$ cathode. the power supply used here has a slower fall-time than in Figure 4.23. 


\subsection{Conclusions}

The addition of small quantities of nitrogen to a 0.8 Torr plasma has revealed the mechanisms of excitation and ionization in the PGD. Optical absorbance and emission measurements demonstrate the transfer of energy from excited argon atoms to nitrogen molecules during the voltage-on period, with a subsequent reduction in the number of metastable states of argon. This reduction in metastables reduces the ionization of sputtered atoms during the voltage-on period, but does not greatly effect emissions from excited analyte atoms because the latter are created mostly via collisions with electrons.

Attenuation factors for $\mathrm{ArH}^{+}$and $\mathrm{Ar}^{2+}$, are more than twice as great as for $\mathrm{Ar}^{+}$, indicating that these ions are either reliant upon two highly energetic precursors (both of which are attenuated by the added nitrogen), or are reliant on one energetic precursor and have an additional destruction pathway with nitrogen. Without further study, the exact mechanisms cannot be determined.

When the voltage is terminated in the 'pure' argon discharge, ToF-MS data and optical experiments show that argon ion recombination leads to an increase in metastable states. This, in-turn, leads to an increase in the propensity for Penning ionization. Metal ions and emission from recombining metal ions are observed for several milliseconds after pulse termination. When added, nitrogen prevents electrons from collisionally cooling in the afterglow due to superelastic collisions with vibrationally excited states formed during the voltage-on period. These collisions delay the onset for recombination because fast electrons recombine less-readily than slow electrons. During this delayed electron-cooling period, ions and electrons are lost by diffusion to the walls with the result that fewer recombination reactions occur when the conditions are eventually 
permissible. The afterpeak delay, and magnitude, is shown to be controllable by the nitrogen partial pressure. The reduction in afterpeak emissions caused by the addition of nitrogen could provide a lower background environment for AAS and AFS experiments, while allowing the AAS and AFS measurements to be made closer in time to the voltageoff period. This would allow measurements to be made at a time of maximum sputtered atom density - as they will not have had time to diffuse away. Also, the ability for nitrogen to quickly remove argon ions and delay the onset for the production of sputtered analyte ions could be particularly useful for reducing the interference of discharge-gas species in time-gated MS measurements. ${ }^{48,}{ }^{49}$ While nitrogen was intended to be used only as a diagnostic tool in these experiments, it turns out that controlled additions of nitrogen to PGDs might actually be highly beneficial for time-gated AAS, AFS (and laser-induced atomic fluorescence (LIAF)) and MS analytical methods. 


\subsection{References}

(1) Kano, K.; Suzuki, M.; Akatsuka, H. Plasma Sources Sci. Technol. 2000, 9, 314322.

(2) Fujimoto, T. J. Phys. Soc. Japan 1979, 47, 265-272.

(3) Fujimoto, T. J. Phys. Soc. Japan 1980, 49, 1569-1576.

(4) van der Mullen, J. A. M. Phys. Rev. 1990, 191, 109-220.

(5) Valiga, R. E.; Duckworth, D. C.; Smith, D. H. Rapid Commun. Mass Spectrom. 1996, $10,305-310$.

(6) De Gendt, S.; Van Grieken, R. E.; Ohorondnik, S. K.; Harrison, W. W. Anal. Chem. 1995, 67, 1026-1033.

(7) Hoppstock, K.; Harrison, W. W. Anal. Chem. 1995, 67, 3167-3171.

(8) Ohorodnik, S. K.; Harrison, W. W. Anal. Chem. 1993, 65, 2542-2544.

(9) Ohorodnik, S. K.; Harrison, W. W. J. Anal. At. Spectrom. 1994, 9, 991-996.

(10) Ratliff, P. H.; Harrison, W. W. Spectrochim. Acta, Part B 1994, 49, 1747-1757.

(11) Coburn, J. W. Rev. Sci. Instr. 1970, 41, 1219-1223.

(12) Knewstubb, P. F.; Tickner, A. W. J. Chem. Phys. 1962, 36, 684-693.

(13) Loving, T. J.; Harrison, W. W. Anal. Chem. 1983, 55, 1526-1530.

(14) Ratliff, P. H.; Harrison, W. W. Appl. Spectrosc. 1995, 49, 863-871.

(15) Wagatsuma, K. Spectrochim. Acta, Part B 2001, 56, 465-486.

(16) Wagatsuma, K.; Hirokawa, K. Anal. Chem. 1985, 57, 2901-2907.

(17) Wagatsuma, K.; Hirokawa, K. Anal. Chem. 1989, 61, 326-329.

(18) Wagatsuma, K.; Danzaki, U. J. Anal. At. Spectrom. 1999, 14, 1727-1730. 
(19) Wagatsuma, K.; Hirokawa, K. Spectrochim. Acta, Part B 1988, 43, 831-839.

(20) Wagatsuma, K.; Hirokawa, K. Spectrochim. Acta, Part B 1987, 42, 523-531.

(21) Pollmann, D.; Ingeneri, K.; Harrison, W. W. J. Anal. At. Spectrom. 1996, 11, 849853.

(22) Fishburne, E. S. J. Chem. Phys. 1967, 47, 58-63.

(23) Imura, K.; Midorikawa, R.; Kasai, T.; Ohoyama, H.; Che, D. Chem. Lett. 1996, 299-300.

(24) Prince, J. F.; Collins, C. B.; Robertson, W. W. J. Chem. Phys. 1964, 40, 26192626.

(25) Nguyen, T. D. Chem. Phys. 1983, 79, 41-55.

(26) Calo, J. M.; Axtmann, R. C. J. Chem. Phys. 1971, 54, 4961-4963.

(27) Coburn, J. W.; Kay, E. Appl. Phys. Lett. 1971, 18, 435-438.

(28) Eckstein, E. W.; Coburn, J. W.; Kay, E. Int. J. Mass Spectrom. Ion Physics 1975, 17, 129-138.

(29) Barshick, C. M. In Inorganic Mass Spectrometry; Barshick, C. M., Duckworth, D. C., Smith, D. H., Eds.; Marcel Dekker, Inc.: New York, 2000; Vol. 23.

(30) Chapman, B. Glow Discharge Processes; John Wiley and Sons: New York, 1980.

(31) Duckworth, D. C.; Barshick, C. M. Anal. Chem. 1998, 70, 709A-717A.

(32) Duckworth, D. C.; Marcus, R. K. Appl. Spectrosc. 1990, 44, 649-655.

(33) Harrison, W. W.; Hess, K. R.; Markus, R. K.; King, F. L. Anal. Chem. 1986, 58, 341A-356A.

(34) King, F. L.; Harrison, W. W. Mass Spec. Rev. 1990, 9, 285-213.

(35) King, F. L.; Teng, J.; Steiner, R. E. J. Mass Spec. 1995, 30, 1061-1075. 
(36) Marcus, R. K. Glow Discharge Spectroscopies, 1 ed.; Plenum Press, 1993.

(37) Stuewer, D. Fresnius' J. Anal. Chem. 1990, 337, 737-742.

(38) Bogaerts, A.; Gijbels, R. Phys. Rev. A 1995, 52, 3743-3751.

(39) Bogaerts, A.; Guenard, R. D.; Smith, B. W.; Winefordner, J. D.; Harrison, W. W.; Gijbels, R. Spectrochim. Acta, Part B 1997, 52, 219-229.

(40) Biondi, M. A. Phys. Rev. 1952, 88, 660-665.

(41) Knewstubb, P. F.; Tickner, A. W. J. Chem. Phys. 1962, 36, 674-683.

(42) Cermak, V. J. Chem. Phys. 1966, 44, 3781-3786.

(43) Jones, E. G.; Harrison, A. G. Int. J. Mass Spectrom. Ion Physics 1970, 5, 137-156.

(44) Stedman, D. H.; Setser, D. W. J. Chem. Phys. 1970, 52, 3957-3965.

(45) Hang, W.; Baker, C.; Smith, B. W.; Winefordner, J. D.; Harrison, W. W. J. Anal. At. Spectrom. 1997, 12, 143-149.

(46) Harrison, W. W.; Hang, W. J. Anal. At. Spectrom. 1996, 11, 835-840.

(47) Su, Y.; Yang, P.; Zhou, Z.; Wang, X.; Li, F.; Huang, B.; Ren, J.; Chen, M.; Ma, H.; Zhang, G. Spectrochim. Acta, Part B 1997, 53, 1413-1420.

(48) Solyom, D. A.; Hieftje, G. M. J. Anal. At. Spectrom. 2002, 17, 329-333.

(49) Lewis, C. L.; Oxley, E. S.; Pan, C. K.; Steiner, R. E.; King, F. L. Anal. Chem. 1999, 71, 230-234.

(50) Pan, C.; King, F. L. Anal. Chem. 1993, 65, 3187-3193.

(51) Pan, C.; King, F. L. J. Am. Soc. Mass Spectrom. 1993, 4, 727-732.

(52) King, F. L.; Pan, C. Anal. Chem. 1993, 65, 735-739.

(53) Pan, C.; King, F. L. Appl. Spectrosc. 1993, 47, 300-304.

(54) Steiner, R. E.; Lewis, C. L.; King, F. L. Anal. Chem. 1997, 69, 1715-1721. 
(55) Riseberg, L. A.; Parks, W. F.; Schearer, L. D. Phys. Rev. A 1973, 8, 1962-1968.

(56) Ferreira, N. P.; Strauss, J. A.; Human, H. G. C. Spectrochim. Acta, Part B 1982, 37, 273-279.

(57) Pan, C.; King, F. L. Appl. Spectrosc. 1993, 47, 2096-2101.

(58) Wiese, W. L. Spectrochim. Acta, Part B 1991, 46, 831-841.

(59) West, C. D.; Human, H. G. C. Spectrochim. Acta, Part B 1976, 31, 81-92.

(60) Ferreira, N. P.; Human, H. G. C.; Butler, L. R. P. Spectrochim. Acta, Part B 1980, $35,287-295$.

(61) Piepmeier, E. H.; deGalan, L. Spectrochim. Acta, Part B 1975, 30, 263-279.

(62) Hugon, R.; Henrion, G.; Fabry, M. Meas. Sci. Technol. 1996, 7, 553-559.

(63) Phelps, A. V.; Molnar, J. P. Phys. Rev. 1953, 89, 1202-1208.

(64) Setser, D. W.; Stedman, D. H.; Coxon, J. A. J. Chem. Phys 1970, 53, 1004-1020.

(65) Bourene, M.; LeCalve, J. J. Chem. Phys 1973, 58, 1452-1458.

(66) Piper, L. G.; Velazco, J. E.; Setser, D. W. J. Chem. Phys. 1973, 59, 3323-3340.

(67) LeCalve, J.; Bourene, M. J. Chem. Phys. 1973, 38, 1446-1451.

(68) Velazco, J. E.; Kolts, J. H.; Setser, D. W. J. Chem. Phys. 1978, 69, 4357-4373.

(69) Copley, G. H.; Lee, C. S. Can. J. Phys. 1975, 53, 1705-1714.

(70) Ferreira, C. M.; Loureiro, J.; Ricard, A. J. Appl. Phys. 1985, 57, 82-90.

(71) Calo, J. M.; Axtmann, R. C. J. Chem. Phys. 1971, 54, 1332-1341.

(72) Johnson, A. W.; Fowler, R. G. J. Chem. Phys 1970, 53, 65-72.

(73) Jackson, G. P.; Lewis, C. L.; Doorn, S.; Majidi, V.; King, F. L. Spectrochim. Acta, Part B 2001, 56, 2449-2464.

(74) Bogaerts, A.; Gijbels, R. J. Anal. At. Spectrom. 2001, 16, 239-249. 
(75) Bogaerts, A.; Gijbels, R. Spectrochim. Acta 1998, 53B, 1-42.

(76) Wagatsuma, K.; Hirokawa, K. Anal. chem. 1989, 61, 326-329.

(77) Adams, N. G.; Dean, A. G.; Smith, D. Int. J. Mass Spectrom. Ion processes 1972/73, 10, 63-76.

(78) McDaniel, E. W.; Cermak, V.; Dalgarno, A.; Ferguson, E. E.; Friedman, L. IonMolecule Reactions, 10 ed.; John Wiley \& Sons: New York, 1970.

(79) Biondi, M. A. Phys. Rev. 1963, 129, 1181-1188.

(80) Kasner, W. H.; Biondi, M. A. Phys. Rev. A 1965, 137, 317-321.

(81) Kenty, C. Phys. Rev. 1928, 32, 624-635.

(82) Kano, K.; Akatsuka, H. Jpn. J. Appl. Phys. 2001, 40, 4701-4708.

(83) Dyatko, N. A.; Ionikh, Y. Z.; Kolokolov, N. B.; Meshchanov, A. V.; Napartovich, A. P. J. Phys. D: Appl. Phys. 2000, 33, 2010-2018.

(84) Dahli, S. K. IEEE Trans. Plasma Sci. 1989, 17, 903-611.

(85) Blaustein, B. D., Ed. Chemical Reactions in Electrical Discharges; American Chemical Society: Washington, D.C., 1969.

(86) Penning, F. M. Physik 1925, 46, 225.

(87) Inaba, S.; Goto, T.; Hattor, S. J. Phys. Soc. Japan 1983, 52, 1164-1167.

(88) Lide, D. R., Ed. CRC Handbook of Chemistry and Physics., 81 ed.; CRC Press: New York, 2000.

(89) Hecq, M.; Hecq, A. J. Appl. Phys. 1984, 56, 872-873.

(90) Vieth, W.; Huneke, J. C. Spectrochim. Acta, Part B 1990, 45, 941-949.

(91) Fang, D.; Marcus, R. K. Spectrochim. Acta, Part B 1991, 46, 983-1000.

(92) Ferreira, N. P.; Human, H. G. C. Spectrochim. Acta, Part B 1981, 36, 215-229. 
(93) Jacubowski, N.; Feldmann, I.; Steuwer, D. J. Anal. At. Spectrom. 1997, 12, 151157.

(94) Steers, E. B. M.; Fielding, R. J. J. Anal. At. Spectrom. 1987, 2, 239-244.

(95) Wagatsuma, K.; Hirokawa, K. Spectrochim. Acta, Part B 1991, 46, 269-281.

(96) Wagatsuma, K.; Matsuta, H. Spectrochim. Acta, Part B 1999, 54, 527-535.

(97) Yang, C.; Ingeneri, K.; Harrison, W. W. J. Anal. At. Spectrom. 1999, 14, 693-698.

(98) Yang, C.; Harrison, W. W. Spectrochim. Acta, Part B 2001, 56, 1195-1208.

(99) Caroli, S. Prog. Analyt. Atom. Spectrosc. 1983, 6, 253-292.

(100) Marcus, R. K.; King, F. L.; Harrison, W. W. Anal. Chem. 1986, 58, 972-974.

(101) Chakrabarti, C. L.; Headrick, K. L.; Hutton, J. C.; Bicheng, Z.; Bertels, P. C.; Back, M. H. Anal. Chem. 1990, 62, 574-586.

(102) Smith, R. L.; Serxner, D.; Hess, K. R. Anal. Chem. 1989, 61, 1103-1108.

(103) Levy, M. K.; Serxner, D.; Angstadt, A. D.; Smith, R. L.; Hess, K. R. Spectrochim. Acta, Part B 1991, 46, 253-267.

(104) Parker, J. E.; Lehle, R. S. Int. J. Mass Spectrom. Ion Physics 1971, 7, 421-469.

(105) Yan, X.; Ingeneri, K.; Hang, W.; Harrison, W. W. J. Anal. At. Spectrom. 2001, $16,819-824$.

(106) Brackett, J. M.; Mitchell, J. C.; Vickers, T. J. Appl. Spectrosc. 1984, 38, 136-140.

(107) Van Dijk, C.; Smith, B. W.; Winefordner, J. D. Spectrochim. Acta, Part B 1982, 37, 759-768.

(108) Mehs, D. M.; Niemczyk, T. M. Appl. Spec. 1981, 35, 66-69.

(109) Bogaerts, A.; Gijbels, R. Spectrochim. Acta, Part B 1998, 53, 1-42.

(110) Lewis, C. L. Dissertation, West Virginia University, Morgantown, 2001. 
(111) Lewis, C. L.; Jackson, G. P.; Doorn, S. K.; Majidi, V.; King, F. L. Spectrochim. Acta, Part B 2001, 56, 487-501.

(112) Butler, L. R. P.; Kroger, K.; West, C. D. Spectrochim. Acta, Part B 1975, 30, 489499.

(113) Ferreira, N. P.; Strauss, J. A.; Human, H. G. C. Spectrochim. Acta, Part B 1983, 34, 899-911.

(114) Glick, M.; Smith, B. W.; Winefordner, J. D. Anal. Chem. 1990, 62, 157-161.

(115) Smith, B. W.; Omenetto, N.; Winefordner, J. D. Spectrochim. Acta, Part B 1984, 39, 1389-1393.

(116) Womack, J. B.; Gessler, E. M.; Winefordner, J. D. Spectrochim. Acta, Part B 1991, 46, 301-308.

(117) Su, T.; Bowers, M. T. Int. J. Mass Spectrom. Ion Physics 1973, 12, 347-356.

(118) Klema, E. D.; Allen, J. S. Phys. Rev. 1950, 77, 661-665.

(119) Colli, L.; Facchini, U. Rev. Sci. Instrum. 1952, 23, 39-42.

(120) Nielson, R. A. Phys. Rev. 1936, 50, 950-954.

(121) Mason, R. S.; Miller, P. D.; Mortimer, I. P. Phys. Rev. E 1997, 55, 7462-7472.

(122) Lias, S. G.; Liebman, J. F.; Levin, R. D. J. Phys. Chem. Ref. Data 1984, 13, 695808. 


\section{Chapter 5}

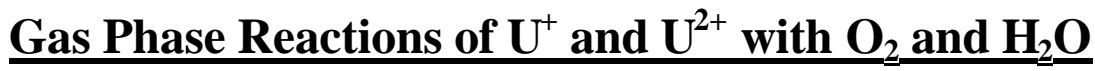 \\ in a Quadrupole Ion Trap}

\subsection{Introduction}

Quadrupole ion traps (QITs) serve as one of the most versatile mass spectrometers available to physicists, chemists and biochemists. It is possible to store ions of either charge, or both charges simultaneously, up to several kilodaltons in mass. ${ }^{1}$ Trapped ions can be dissociated by a variety of methods in order to obtain thermodynamic or structural information. ${ }^{2}$ It is also possible to study ion molecule reactions by substituting part or all of the bath gas with a reagent gas of interest. ${ }^{3}$ Furthermore, by introducing a known quantity of reagent, and allowing a reaction to occur for a known time period, it is possible to determine the rate constant for an ion-molecule reaction. ${ }^{3}$ Bonner et al. were the first to demonstrate the possibility of measuring rate constants in the QIT by studying the charge and proton transfer reactions between small organic molecules. ${ }^{4}$ Such determinations are often dependent on temperature..$^{5}$ To obtain meaningful quantitative data it is important to ascertain the temperature or kinetic energy of the colliding partners. $^{6}$ In ion beam experiments, the acceleration voltage leading to the reaction chamber determines the kinetic energy. In ion traps, however, quantification of the ions' kinetic energy is somewhat more complicated-especially when a bath gas is introduced into the trapping region to dampen the kinetic energy of the ions. 
Numerous investigators have reported reaction rate constants of gas-phase ionmolecule reactions and the mean kinetic energies associated with trapped ions. ${ }^{4-17}$ These reactions were conducted almost exclusively in helium. The general conclusions are that the kinetic energy of trapped ions increases as a function of increasing trapping potential and decreasing bath gas pressure. An increase in kinetic energy can either increase or decrease the rate of a reaction depending on the thermodynamics involved. Rates of exothermic reactions often decrease with increasing kinetic energy, whereas the rates of endothermic reactions tend to increase with increasing kinetic energy.

The presence of a bath gas to buffer the ion kinetic energy is useful for reducing the influence of the trapping potential. Helium is the most commonly used bath gas, at pressures of $\sim 0.1-5 \times 10^{-3}$ Torr. At this time, no direct comparisons of different bath gases have been published to determine how the gases might affect the kinetic energy or internal energy of the ions in the QIT. It has been shown, however, that increasing the partial pressure of $\mathrm{N}_{2}$ in a constant trap pressure of 1.7 mTorr $\mathrm{He} / \mathrm{N}_{2}$ can increase the internal energy of a molecular ion. ${ }^{16}$ It is not known to what extent this observation is related to the center of mass collision energy or collision frequency etc. This manuscript will present simulations in order to predict the effect of using a bath gas heavier than helium.

Recent studies in our laboratory measured the kinetics of collision induced dissociation (CID) of strongly bound metal oxides $\left(\mathrm{D}_{0}>800 \mathrm{~kJ} \mathrm{~mol}^{-1}\right)$ in QITs. ${ }^{18-20}$ To dissociate these strongly bound oxides, experiments were performed with neon (in place of helium) in order to achieve the high internal temperatures required. The metal oxides studied were formed via metal ion reactions with $\mathrm{O}_{2}$ in the trap. Because very few studies 
have considered the effect of a heavier bath gas on reaction rates, it was questioned how well the oxidation rates could be measured in the QIT using neon as the bath gas. The reaction rates of uranium ions with $\mathrm{O}_{2}$ are measured here and compared with literature values to assess the accuracy of this method. The approach is also used to probe other reaction rates.

Ion isolation and double resonance ejection experiments are also used to probe reaction pathways and thermodynamics of uranium ions with oxygen and water. The ability to detect and eject ions, selectively, at $0.5 \mathrm{~m} / \mathrm{z}$ resolution reveals a previously unknown gas-phase oxidation pathway for the doubly charged uranium ion with water.

\subsection{Experimental apparatus and method of measurement}

The pulsed glow discharge ion trap mass spectrometer used in this study has been described in detail elsewhere. ${ }^{21}$ A uranium pin of natural isotopic abundance served as the sample cathode and was positioned on a direct insertion probe. ${ }^{22}$ Neon was used as the glow discharge support gas at a pressure of 0.8 Torr and as the ion trap bath gas at 0.5 mTorr. A leak valve (Series 203, Granville-Phillips, Boulder, CO) introduced compressed oxygen (Air Liquide) or water (vapor) at pressures between $9 \times 10^{-7}$ and $7 \times 10^{-6}$ Torr. Isotopically enriched $\mathrm{H}_{2}{ }^{18} \mathrm{O}$ (66 atom $\%{ }^{18} \mathrm{O}$, Isotec Inc., Miamisburg, $\mathrm{OH}$ ) was used to verify the source of oxygen in the reactions involving water. An ion gauge (Vacuum Instrument Corporation, Ronkonkoma, NY) measured the pressure of oxygen (or water) before the addition of the neon bath gas and after each experiment when the neon had been evacuated. 
The reaction time for the isolated ion of interest and the reagent molecule is defined as the time between the last isolation event in the scan function and the initiation of the mass spectrum. In reality, ions will continue to react during the scan function until such time as they are ejected and detected. But because the reagent ions are always the lightest (in these experiments) and the first to be ejected in the scan function (after $<2$ $\mathrm{ms}$ ), no correction is necessary in these experiments to account for the reactions occurring during the acquisition period. An automated "experiment editor" varied the reaction period so that in a typical experiment, 16 mass spectra were averaged to obtain intensity measurements at $2 \mathrm{~ms}$ intervals from 0 to $50 \mathrm{~ms}$ reaction time. Plots of the reactant ion signal intensity versus time were thereby generated. The rates were verified to be pseudo-first order so that the temporal plots could be converted to a phenomenological rate, $n k$, according to the expression

$$
-\ln \left(\frac{\left[M^{+}\right]_{t}}{\left[M^{+}\right]_{0}}\right)=n k t
$$

where $n$ is the number density of the reagent gas, $\left[\mathrm{M}^{+}\right]_{0}$ is the initial ion signal of reagent ion, and $\left[\mathrm{M}^{+}\right]_{t}$ is the reagent ion signal at time $t$. This equation also assumes a linear relationship between ion signal, as measured by the ion detector, and ion number density (a valid assumption over small ranges).

By repeating the experiment at various values of $n$ (different reagent gas pressures) the pseudo-first order rate constant $k$ can be obtained from the slope of the plot $n k$ versus $n$. If the rate constant for a given reaction is known, the measured rates could be used to calibrate the ion gauge. ${ }^{7}$ The fitted slopes of $n k$ versus $n$ are not forced through zero because reactions are observed to occur when no reagent gases are added 
(i.e. $n$ is actually a sum of $n_{\text {residual }}$ and $n_{\text {added }}$, where $n_{\text {residual }}$ is the residual number density of $\mathrm{O}_{2}$ arising from contaminants in the vacuum system). Quantification of the initial reagent concentration, $n_{\text {residual }}$, is possible, by determining the $\mathrm{x}$ intercept of the slopes in Figures 4 and 5. In this study, a complication in the determination of $n_{\text {residual }}$ for $\mathrm{O}_{2}$ is that trace levels of $\mathrm{H}_{2} \mathrm{O}$ are also present (in larger abundance) and contribute to the oxidation reactions of uranium ions. In these experiments, we assume that the residual pressures of $\mathrm{O}_{2}$ and $\mathrm{H}_{2} \mathrm{O}$ remain constant throughout, and therefore do not affect the determination of the rate constants from the slopes. This assumption is supported by the correlation of the least squares curve fitting.

\subsection{Results and discussion}

\subsubsection{Kinetic energy considerations}

According to previous reports, the mean kinetic energy of an ion in the ion trap will be approximately $0.1-2 \mathrm{eV}$ following $\sim 15$ collisions in $\sim 1-5 \times 10^{-3}$ Torr He. $^{12-14}$ When a bath gas is present, the kinetic energy of the ions increases only slightly with increasing trapping potential until a stability boundary is approached. Because most experiments are performed in helium, very little is reported about the effects of using a heavier bath gas such as neon. To obtain a better understanding of the kinetics of ions trapped in a neon bath gas at different trapping potentials, calculations were performed using ITSIM software. ${ }^{23}$ For comparison, calculations were performed with both helium and neon as bath gases. The simulation modeled $500{ }^{238} \mathrm{U}$ ions at a pressure of $1 \mathrm{mT}$ Torr bath gas at $300 \mathrm{~K}$. In Figure 1, the root mean squared (RMS) kinetic energy of $\mathrm{U}^{+}$in neon is plotted as a function of $\mathrm{q}_{\mathrm{z}}$ to show that once steady state conditions are established 
there is very little difference in RMS kinetic energy between $0.1 \leq \mathrm{q}_{\mathrm{z}} \leq 0.65$. At steady state, no significant difference is found between helium and neon as bath gases (data not shown). At a $\mathrm{q}_{\mathrm{z}}$ of 0.67 , at which the presented experiments are conducted, the calculated RMS kinetic energy is $\sim 0.13 \mathrm{eV}$.

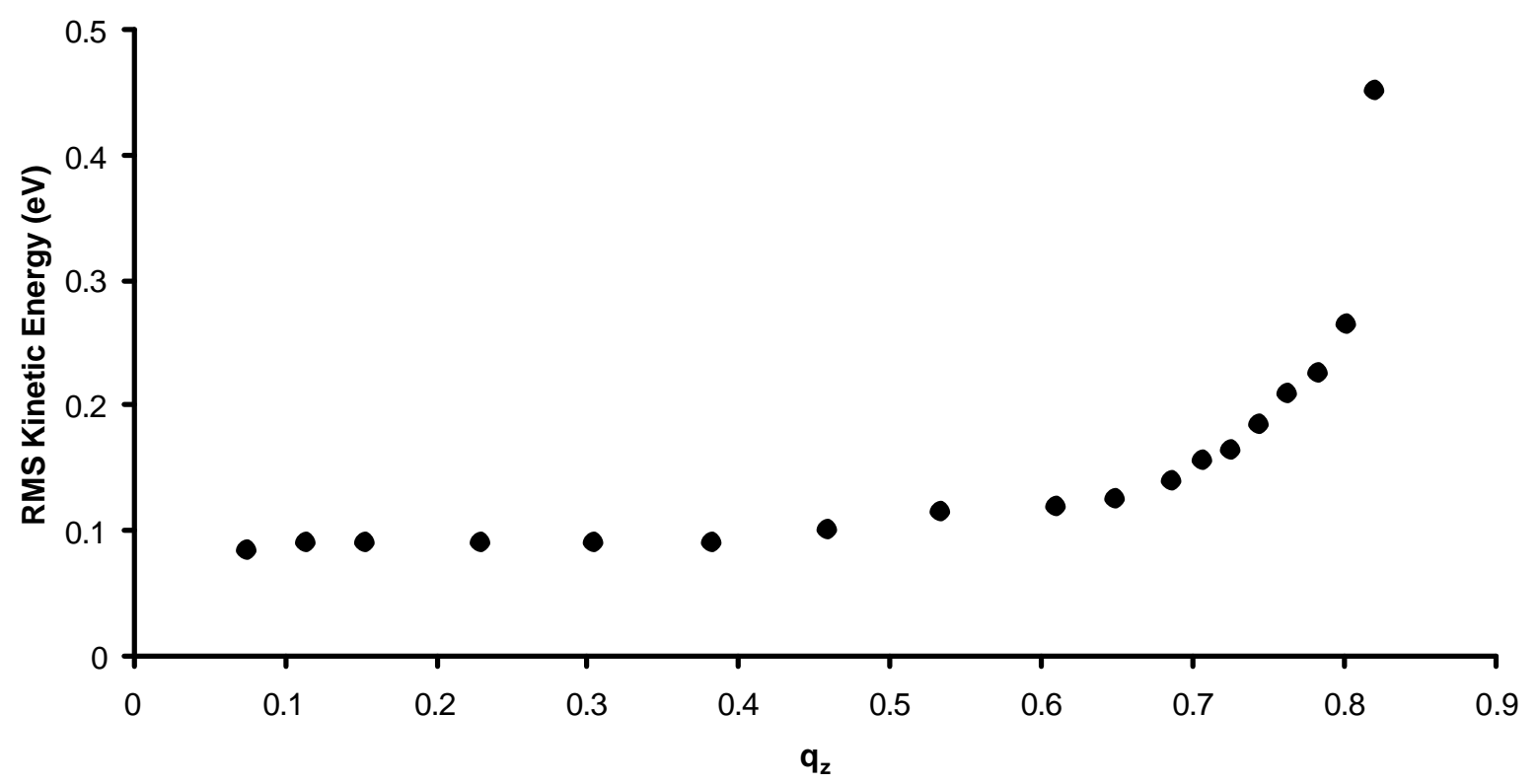

Fig. 5.1. Simulated root mean square (RMS) ion kinetic energy of uranium ions as a function of Mathieu parameter $\mathrm{q}_{\mathrm{z}}(1 \mathrm{mTorr} \mathrm{Ne})$. 
As a first approximation, the temperature for the measured rate coefficients is given by the effective reaction temperature, $T_{e f f}^{R x n},{ }^{24}$

$$
T_{e f f}^{R x n}=T_{b u f}+\frac{m_{R}}{3 \cdot k_{b}} v_{d}^{2}\left(\frac{m_{i o n}+m_{b u f}}{m_{i o n}+m_{R}}\right)
$$

where $\mathrm{T}_{\text {buf }}$ is the buffer gas temperature, $v_{d}$ is the velocity of the ion packet, $k_{b}$ is the Boltzmann constant, and $m_{i o n}, m_{b u f}, m_{R}$ are the masses of the ion, buffer, and reutral reactant, respectively. A good estimate of $v_{d}$ can be obtained from the Wannier equation $^{25}$ for the total laboratory kinetic energy, $K E_{l a b}$.

$$
K E_{\text {lab }}=\frac{3}{2} k_{b} T_{b u f}+\frac{1}{2} m_{i o n} v_{d}^{2}+\frac{1}{2} m_{b u f} v_{d}^{2}
$$

The first term on the right hand side is due to the thermal energy of the buffer gas, the second term results from the velocity of the ion packet, and the last term is due to conversion of ion drift motion to random motion via collisions with the buffer gas.

Because ions generally were stored at low trapping potentials for at least 20-25 $\mathrm{ms}$ before the reaction period, they are assumed to be kinetically cooled to approximately the calculated RMS kinetic energy. For the reaction, $\mathrm{U}^{+}+\mathrm{O}_{2}$, making the approximation $K E_{\text {lab }} \approx 0.13 \mathrm{eV}$, solving equation 3 for $v_{d}$, and substituting into equation 2 yields $T_{e f f}^{R x n} \approx 384 \mathrm{~K}$; the corresponding mean center-of-mass kinetic energy of the reactants, $K E_{C O M}^{R x n}=\frac{3}{2} k_{b} T_{\text {eff }}^{R x n}$, is $\sim 0.050 \mathrm{eV}\left(4.8 \mathrm{~kJ} \mathrm{~mol}^{-1}\right)$. The maximum internal energy of the neutral reactant is equal to $K E_{C O M}^{R x n}$ (only electronic excitations are possible for atomic ions), thus implying that the observed reactions are unlikely to be endothermic by more than $\sim 5 \mathrm{~kJ} \mathrm{~mol}^{-1}$, which is well within the error of most reported enthalpies. For the case of diatomic ions under similar reaction conditions, the maximum internal energy (now 
partitioned between ions and neutral reactants) is also $K E_{C O M}^{R x n}$, and therefore does not contribute significantly to the energy available for activation/reaction. In all the reactions considered here, two moles of reagents react to form two moles of products, so entropy effects are negligibly small (at near-room-temperature conditions) and are therefore ignored.

\subsubsection{Reactions with $\mathrm{O}_{2}$}

Figure 2 summarizes the reaction pathways examined in this study that involve molecular oxygen. Shown next to each arrow is the measured rate constant (x $10^{-10} \mathrm{~cm}^{3} \mathrm{~s}^{-1}$ ). The rate constants for reactions of singly charged uranium ions with oxygen have been known for some time, ${ }^{26}$ but reaction rate constants for the doubly charged species have been measured only recently. ${ }^{27}$ Tables 1-3 provide thermodynamic and kinetic data both from literature and from results derived in this work. Enthalpies of formation in Table 1 (with the exception of $\mathrm{UO}^{2+}$ and $\mathrm{UOH}^{2+}$ ) were used to calculate the enthalpies of reactions in Tables 2 and 3. For $\mathrm{UO}^{2+}$ and $\mathrm{UOH}^{2+}$, limits for the enthalpies of formation were determined from the exothermic reactions observed and reported in Tables 2 and 3. Other values necessary for the calculations (e.g. $\Delta \mathrm{H}_{\mathrm{f}}(\mathrm{O}), \Delta \mathrm{H}_{\mathrm{f}}\left(\mathrm{H}_{2} \mathrm{O}\right)$ ) are taken from reference 28 with the exception of the gas-phase proton affinity of water that is taken from reference 29 . 


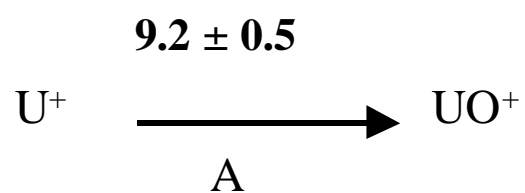

A

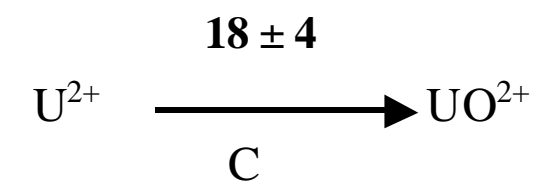

$$
\stackrel{9.0 \pm 0.6}{\longrightarrow} \mathrm{UO}_{2}{ }^{+}
$$

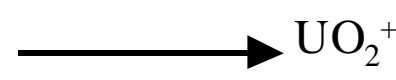

B

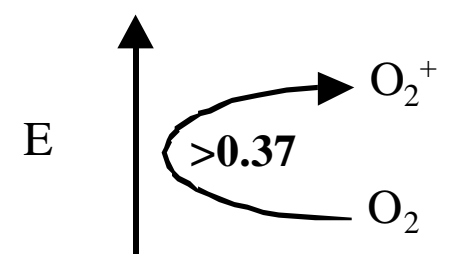

$\mathbf{0 . 3 7} \pm \mathbf{0 . 0 1}$

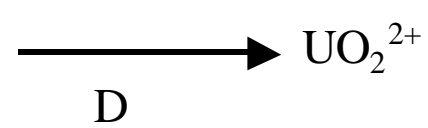

Fig. 5.2. Measured reaction rates and pathway for the reactions of $U^{2+}$ and $U^{+}$with $\mathrm{O}_{2}$. Rates shown have units $10^{-10} \mathrm{~cm}^{3} \mathrm{~s}^{-1}$.

Table 5.1. Thermochemical data of species of interest.

\begin{tabular}{c|c} 
Species (M) & $\Delta \mathrm{H}_{\mathrm{f}} \mathrm{M}_{(\mathrm{g})}\left(\mathrm{kJ} \mathrm{mol}^{1}\right)$ \\
\hline \hline $\mathrm{U}^{2+}$ & $2277 \pm 50^{30}$ \\
$\mathrm{UO}^{2+}$ & $<2047^{31}$ \\
& $<1747^{\mathrm{a}}$ \\
$\mathrm{UOH}^{2+}$ & $1410-1818^{\mathrm{a}}$ \\
$\mathrm{UO}_{2}{ }^{2+}$ & $1553 \pm 250^{31}$ \\
$\mathrm{U}^{+}$ & $1129 \pm 4^{28,30}$ \\
$\mathrm{UO}^{+}$ & $582^{30}$ \\
$\mathrm{UOH}^{+}$ & $516 \pm 35^{32}$ \\
& $54 \pm 50^{30}$ \\
$\mathrm{UO}_{2}{ }^{+}$ & $57^{28,30}$ \\
& $88 \pm 20^{32}$ \\
$\mathrm{O}$ & $249 \pm 0.1^{28}$
\end{tabular}

${ }^{a}$ This work 
Table 5.2. Reactions of singly charged uranium species with molecular oxygen and water.

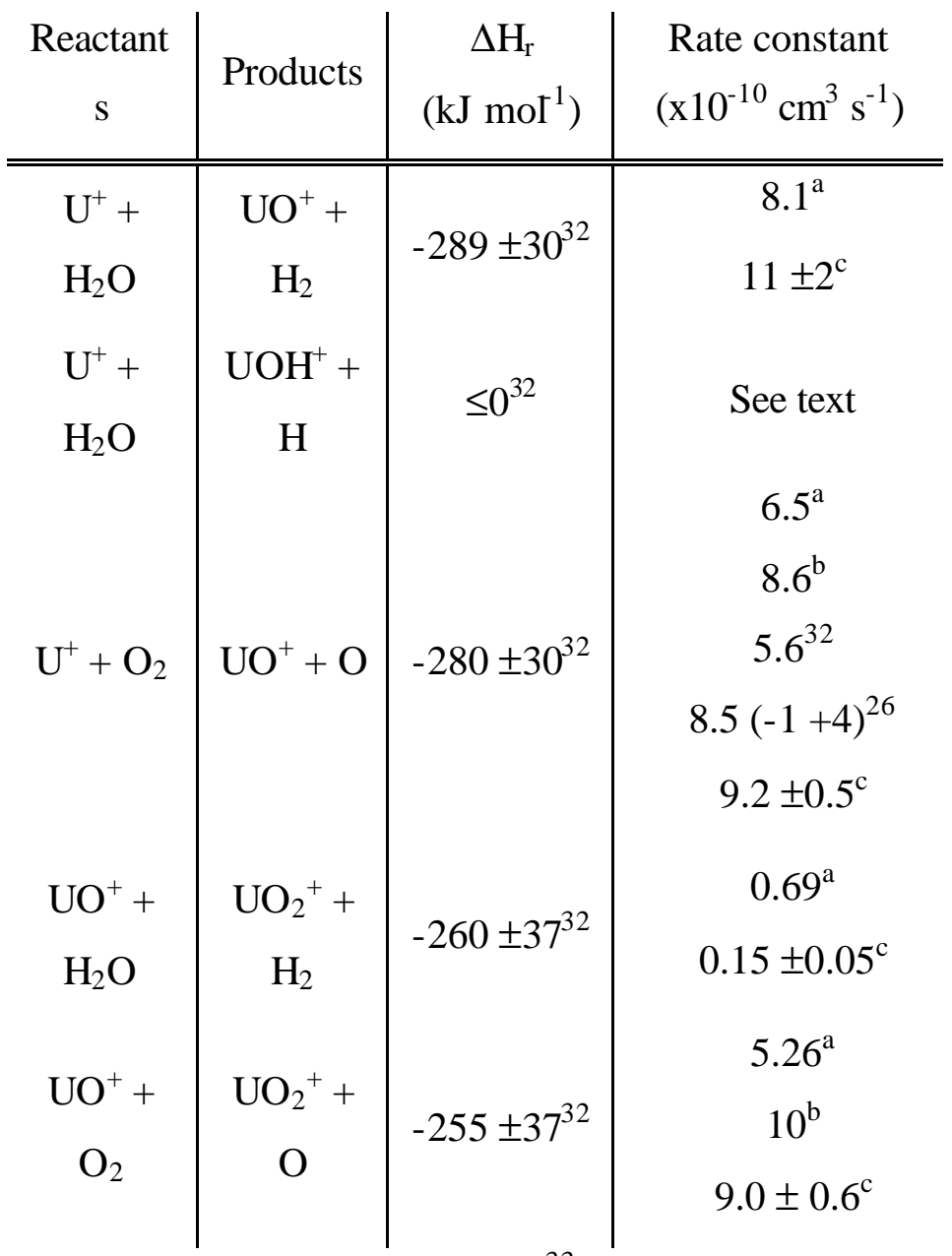

${ }^{\text {a }}$ Calculated from reference 30 using $k_{A D O}$ theory $^{33}$ and values from reference 28.

${ }^{\mathrm{b}}$ Calculated from decay constants in reference 27 assuming $20 \%$ partial pressure of $\mathrm{O}_{2}$ in air at $4 \times 10^{-5}$ Torr air pressure.

${ }^{c}$ This work 
Table 5.3. Reactions of doubly charged uranium species with molecular oxygen and water.

\section{${ }^{a}$ Calculated}

\begin{tabular}{|c|c|c|c|}
\hline Reactants & Produc ts & $\begin{array}{c}\Delta \mathrm{H}_{\mathrm{r}} \\
\left(\mathrm{kJ} \mathrm{mol}^{-1}\right)\end{array}$ & $\begin{array}{l}\text { Rate constant } \\
\left(\mathrm{x} 10^{-10} \mathrm{~cm}^{3} \mathrm{~s}^{-1}\right)\end{array}$ \\
\hline $\mathrm{U}^{2+}+\mathrm{H}_{2} \mathrm{O}$ & $\mathrm{UO}^{2+}+\mathrm{H}_{2}$ & $\begin{array}{l}<11^{\mathrm{a}} \\
\leq 0^{\mathrm{d}}\end{array}$ & $10^{\mathrm{d}}$ \\
\hline $\mathrm{U}^{2+}+\mathrm{H}_{2} \mathrm{O}$ & $\mathrm{UOH}^{2+}+\mathrm{H}$ & $\leq 0^{\mathrm{d}}$ & \\
\hline $\mathrm{U}^{2+}+\mathrm{O}_{2}$ & $\mathrm{UO}^{2+}+\mathrm{O}$ & $\leq 0^{27,31, d}$ & $\begin{array}{l}11^{\mathrm{c}} \\
13^{\mathrm{b}} \\
18 \pm 4^{\mathrm{d}}\end{array}$ \\
\hline $\mathrm{UO}^{2+}+\mathrm{H}_{2} \mathrm{O}$ & $\mathrm{UO}_{2}{ }^{2+}+\mathrm{H}_{2}$ & $\begin{array}{c}\text { Not } \\
\text { observed }\end{array}$ & $<0.1^{\mathrm{d}}$ \\
\hline $\mathrm{UO}^{2+}+\mathrm{H}_{2} \mathrm{O}$ & $\begin{array}{c}\mathrm{UO}^{2+}+ \\
\mathrm{H}_{2} \mathrm{O}^{+}\end{array}$ & $\begin{array}{c}\text { Not } \\
\text { observed }\end{array}$ & $<0.1^{\mathrm{d}}$ \\
\hline $\mathrm{UO}^{2+}+\mathrm{O}_{2}$ & $\mathrm{UO}_{2}^{+}+\mathrm{O}$ & $\leq 0^{27,31, \mathrm{~d}}$ & $\begin{array}{l}0.44^{\mathrm{c}} \\
0.3^{\mathrm{b}} \\
0.37 \pm 0.1^{\mathrm{d}}\end{array}$ \\
\hline $\mathrm{UO}^{2+}+\mathrm{O}_{2}$ & $\mathrm{UO}^{+}+\mathrm{O}_{2}^{+}$ & $>0^{c, 27,31}$ & $<0.37 \pm 0.1^{\mathrm{d}}$ \\
\hline $\begin{array}{c}\mathrm{UOH}^{2+}+ \\
\mathrm{H}_{2} \mathrm{O}\end{array}$ & $\mathrm{UO}^{+}+\mathrm{H}_{3} \mathrm{O}^{+}$ & $\leq 0^{\mathrm{d}}$ & \\
\hline $\begin{array}{c}\mathrm{UOH}^{2+}+ \\
\mathrm{H}_{2} \mathrm{O}\end{array}$ & $\begin{array}{c}\mathrm{UOH}^{+}+ \\
\mathrm{H}_{2} \mathrm{O}^{+}\end{array}$ & $-84^{\mathrm{a}}$ & \\
\hline $\mathrm{UO}_{2}^{2+}+\mathrm{O}_{2}$ & $\mathrm{UO}_{2}^{+}+\mathrm{O}_{2}^{+}$ & $\leq 0^{27,31, \mathrm{~d}}$ & $\begin{array}{l}6.2^{\mathrm{b}} \\
3.8^{\mathrm{c}}\end{array}$ \\
\hline
\end{tabular}

${ }^{\mathrm{b}}$ Calculated from decay constants in reference 27 assuming $20 \%$ partial pressure of $\mathrm{O}_{2}$ in air at $4 \times 10^{-5}$ Torr air pressure. 
${ }^{c}$ Calculated from reference ${ }^{31}$ assuming collision rates for $\mathrm{U}^{2+}, \mathrm{UO}^{2+}, \mathrm{UO}_{2}{ }^{2+}$ with $\mathrm{O}_{2}$ to be $\sim 1.1 \times 10^{-11}$ (see the end of section 3.2.1 for details).

${ }^{\mathrm{d}}$ This work

\subsubsection{1. $\mathrm{U}^{+}$reaction with $\mathrm{O}_{2}$}

The thermodynamic and kinetic aspects of Reaction A are well known and this reaction provides a suitable probe system for the ion trap.

$$
\mathrm{U}^{+}+\mathrm{O}_{2} \rightarrow \mathrm{UO}^{+}+\mathrm{O}
$$

Experiments were first conducted to ensure that charge conservation is maintained and that the reaction from $\mathrm{U}^{+}$to $\mathrm{UO}_{2}{ }^{+}$does in fact proceed through the intermediate $\mathrm{UO}^{+}$. Figure 3 is a plot of the ion signal intensities of each species as a function of time for the reaction $\mathrm{U}^{+}+\mathrm{O}_{2}$ at a nominal oxygen number density of $4 \times 10^{10} \mathrm{~cm}^{-3}\left(\sim 1.3 \times 10^{-6}\right.$ Torr $)$. The constant total ion signal demonstrates that charge conservation is maintained. The temporal profiles shown in Figure 3 are indicative of a two-step reaction sequence in which the reaction proceeds through the intermediate $\mathrm{UO}^{+} \cdot{ }^{34}$ Resonance ejection of the intermediate $\mathrm{UO}^{+}$confirmed that all the $\mathrm{U}^{+}$losses were accounted for in the $\mathrm{UO}^{+}$channel. Therefore, the measured decrease in ion signal of $U^{+}$over time can be used to calculate the rate of Reaction A according to Equation 1.

Reaction rates for Reaction A were measured at four different trapping potentials between $0.2 \leq \mathrm{q}_{\mathrm{z}} \leq 0.8$ at four different pressures of $\mathrm{O}_{2}$. The trapping potential had no statistically significant affect on the measured reaction rates for this reaction, based on the external precision of the measurements. This observation is consistent with the simulations presented earlier, whereby the RMS kinetic energy of uranium ions did not significantly increase over this range of trapping potentials. 


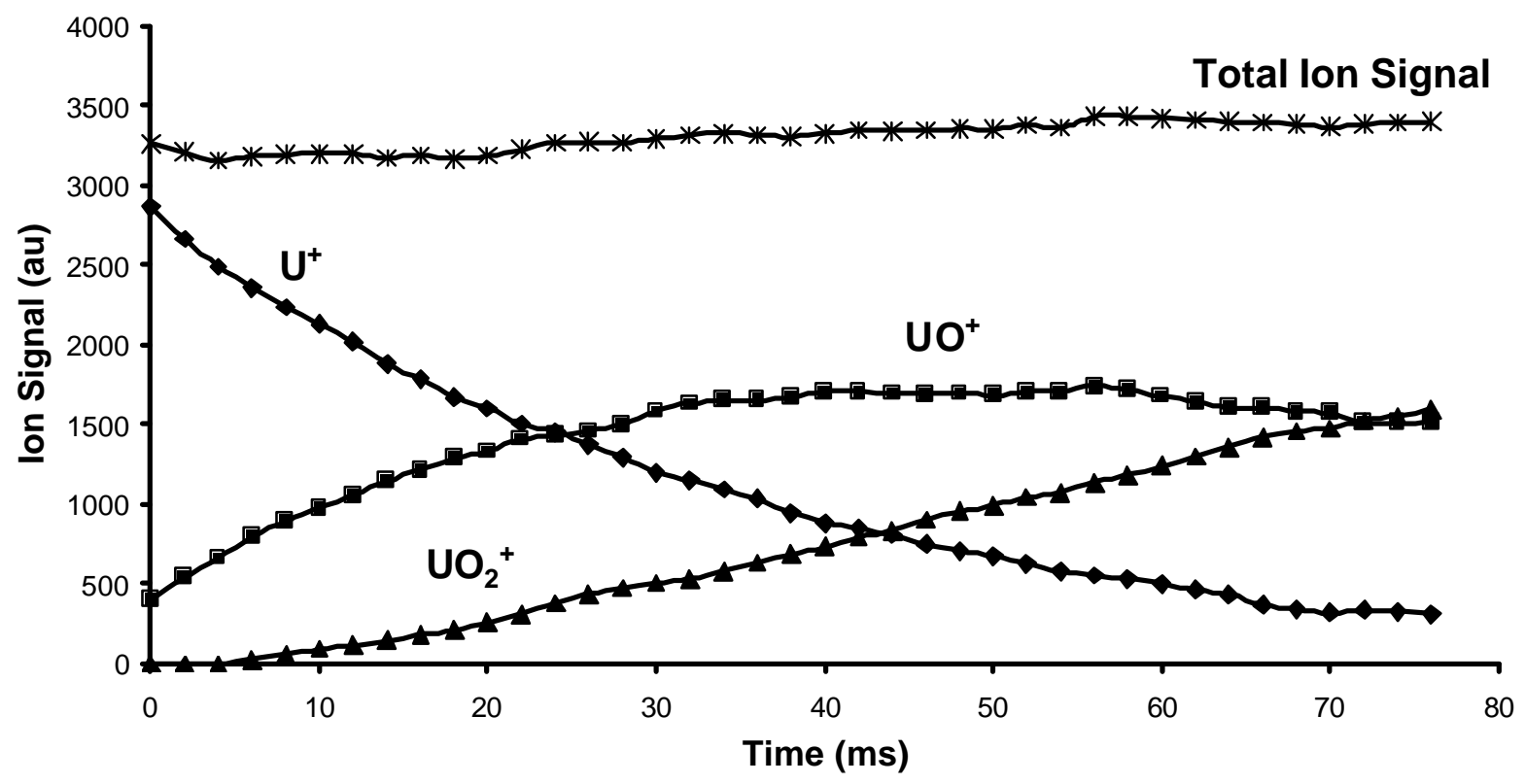

Fig. 5.3. Ion signal intensities versus time for the reaction of $U^{+}$with $\mathrm{O}_{2}\left(1.3 \times 10^{-6}\right.$ Torr $\mathrm{O}_{2}$ ). Bath gas is neon at $0.5 \mathrm{mTorr}, \mathrm{q}_{\mathrm{z}}=0.6$.

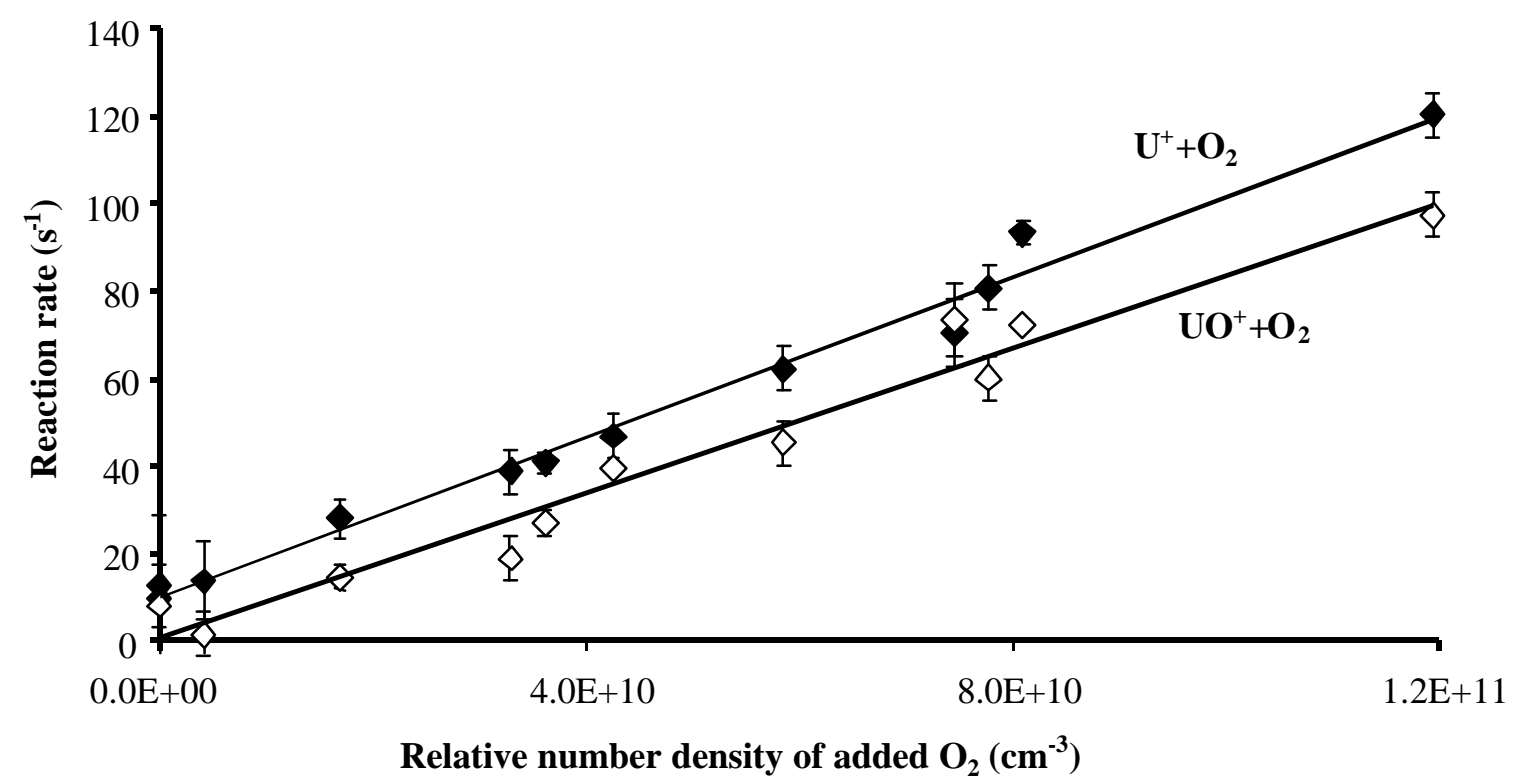

Fig. 5.4. Measured reaction rates of $\mathrm{U}^{+}$(filled diamonds) and $\mathrm{UO}^{+}$(open diamonds) with $\mathrm{O}_{2}$ versus relative number density of added $\mathrm{O}_{2}$. Values shown are the average of four different $\mathrm{q}_{\mathrm{z}}$ values on three separate days with \pm 1 s.d. 
Although this specific reaction is not highly sensitive to kinetic energy effects, the reaction rates have been shown to decrease $(\sim 10 \%)$ as the kinetic energy of the $\mathrm{U}^{+}$ increases (from 0 to $5 \mathrm{eV}$ in lab frame). ${ }^{26} \mathrm{~A}$ reaction that is more sensitive to kinetic effects would be required to examine further the effects of $\mathrm{rf}$ heating at different values of $\mathrm{q}_{\mathrm{z}}$

The reaction rates for $\mathrm{U}^{+}$at several trapping potentials were averaged for each partial pressure of oxygen, measured on three separate days, and are shown in Figure 4 (top curve). A linear regression line reveals a rate constant for Reaction A of $k=9.2 \pm$ $0.5 \times 10^{-10} \mathrm{~cm}^{3} \mathrm{~s}^{-1}(95 \% \mathrm{CL})$. This is in good agreement with the two previous reported values of this reaction of $8.5(-1,+4) \times 10^{-10} \mathrm{~cm}^{3} \mathrm{~s}^{-1}$ by Johnsen and Biondi' ${ }^{26}$ and $8 \times 10^{-}$ ${ }^{10} \mathrm{~cm}^{3} \mathrm{~s}^{-1}$ by Gieray et al. ${ }^{27}$ Cornehl et al. measured this rate as $1.17 k_{A D O} \pm 40 \%{ }^{35}$ and we interpret this to be $k=6.5 \pm 2.6 \times 10^{-10} \mathrm{~cm}^{3} \mathrm{~s}^{-1}$, in reasonable agreement with the other values (to calculate $k_{A D O},{ }^{33}$ the polarizability of $\mathrm{O}_{2}$ was taken from reference 28).

\subsubsection{2. $\mathrm{UO}^{+}$reaction rate with $\mathrm{O}_{2}$}

$$
\mathrm{UO}^{+}+\mathrm{O}_{2} \rightarrow \mathrm{UO}_{2}^{+}+\mathrm{O}
$$

In order to observe Reaction $\mathrm{B}, \mathrm{UO}^{+}$ions were selectively isolated by applying a two-frequency resonance ejection on $\mathrm{U}^{+}$and $\mathrm{UO}_{2}{ }^{+}$, before allowing the reaction with admitted $\mathrm{O}_{2}$ to proceed. Measurements were again made at trapping potentials ranging from $0.2 \leq \mathrm{q}_{\mathrm{z}} \leq 0.8$ and no effect was found on the reaction rates. Figure 4 (lower curve) shows the averages of the rates measured at each $\mathrm{O}_{2}$ pressure for four different trapping potentials. Data collected on three separate days are also included in the error bars $(2 \sigma$, $\mathrm{n} \sim 12$ ) in this plot to demonstrate the day-to-day repeatability of these experiments. The 
difference in the intercepts for the two lines shown in this figure is due to differences in the reactivity of $\mathrm{U}^{+}$and $\mathrm{UO}^{+}$. It was found that $\mathrm{U}^{+}$readily reacts with residual water, while $\mathrm{UO}^{+}$reacts so slowly with water that the product is not observed prior to $\mathrm{O}_{2}$ introduction.

The rate constant for Reaction B is found to be $k=9.0 \pm 0.6 \times 10^{-10} \mathrm{~cm}^{3} \mathrm{~s}^{-1}(95 \%$ CL), the same as that of the bare uranium ion. Gieray et al. report a rate that corresponds to approximately $1 \times 10^{-9} \mathrm{~cm}^{3} \mathrm{~s}^{-1}$, slightly faster than the reported rates for $\mathrm{U}^{+}$. Their experiments were conducted in air, however, and this value assumes a $20 \%$ composition of $\mathrm{O}_{2}$ in air and that no other species interfere with the measurement. Cornehl et al. report a rate of $0.95 k_{A D O} \pm 40 \%{ }^{35}$ and from this we calculate a $k$ of $5.3 \pm 2 \times 10^{-10} \mathrm{~cm}^{3} \mathrm{~s}^{-1}$. No other rate constants for this reaction were found in the extant literature.

\subsubsection{3. $\mathrm{U}^{2+}$ reaction with $\mathrm{O}_{2}$}

$$
\mathrm{U}^{2+}+\mathrm{O}_{2} \rightarrow \mathrm{UO}^{2+}+\mathrm{O}
$$

The pulsed glow discharge ion source generates sufficient quantities of doubly charged ions that their reaction pathways and rates can also be examined. At these low pressures of oxygen it is possible to measure the very fast Reaction C. Figure 5 (top curve) is a plot of the reaction rates of $\mathrm{U}^{2+}$ versus the relative added number density of oxygen. Analysis of the slope gives a rate constant of $1.8 \pm 0.4 \times 10^{-9} \mathrm{~cm}^{3} \mathrm{~s}^{-1}$ (95\% CL). Geiray et al. provide the only other known measurement of this rate as $1.3 \times 10^{-9} \mathrm{~cm}^{3} \mathrm{~s}^{-1}$, again in reasonable agreement. It is interesting to note that although the predicted rate $\left(k_{A D O}=1.1 \times 10^{-9} \mathrm{~cm}^{3} \mathrm{~s}^{-1}\right)$ underestimates the measured rate constant for Reaction $\mathrm{C}$ the predicted rate for the doubly charged uranium is twice the predicted rate of the singly 
charged uranium ion. The measured values for $\mathrm{U}^{+}$and $\mathrm{U}^{2+}\left(9.2\right.$ and $18 \times 10^{-10} \mathrm{~cm}^{3} \mathrm{~s}^{-1}$, respectively) are at least consistent with predicted rates, viz., if both the reactions proceed at the collision rate then the rate for the doubly charged species should be twice that of the singly charged species. ${ }^{33}$

Because this reaction is exothermic, an upper limit of $\Delta \mathrm{H}_{\mathrm{f}}\left(\mathrm{UO}^{2+}\right) \leq 2028 \mathrm{~kJ} \mathrm{~mol}^{-1}$ can be obtained from Reaction $\mathrm{C}$, using values from Table 1. This limit is consistent with the value obtained by Cornehl et al. of $\Delta \mathrm{H}_{\mathrm{f}}\left(\mathrm{UO}^{2+}\right) \leq 2047 \mathrm{~kJ} \mathrm{~mol}^{-1}$, obtained by the reaction of $\mathrm{U}^{2+}$ with $\mathrm{CO}_{2}{ }^{31}$

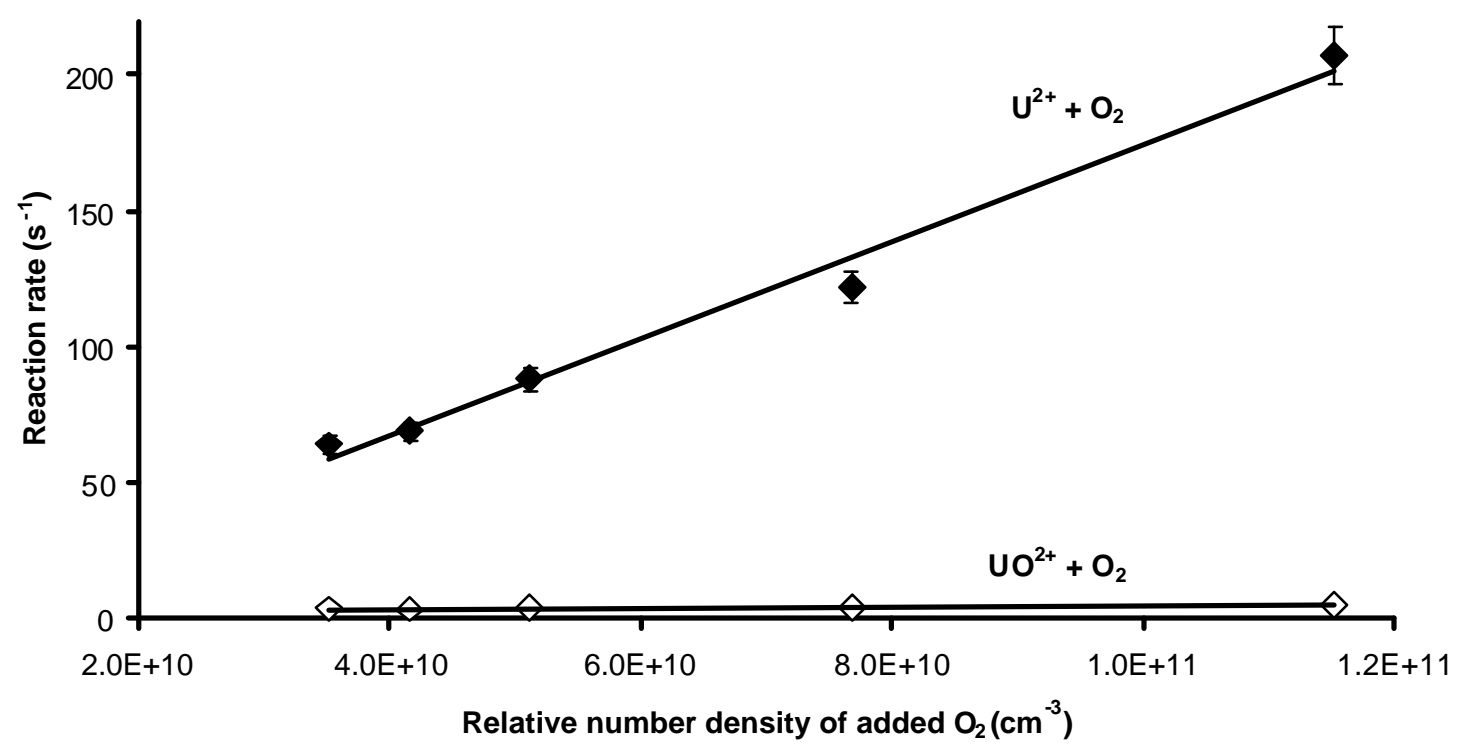

Fig. 5.5. Measured reaction rates versus relative number density of added $\mathrm{O}_{2}$ to determine the rate constants for the reactions of $\mathrm{U}^{2+}$ (filled diamonds) and $\mathrm{UO}^{2+}$ (open diamonds) with $\mathrm{O}_{2}$. Values shown are the average of four different $\mathrm{q}_{\mathrm{z}}$ values on three separate days with \pm 1 s.d. 
5.3.2.4. $\mathrm{UO}^{2+}$ reaction with $\mathrm{O}_{2}$

$$
\mathrm{UO}^{2+}+\mathrm{O}_{2} \rightarrow \mathrm{UO}_{2}^{2+}+\mathrm{O}
$$

Reaction $\mathrm{D}$ is found to proceed with a pseudo-first order rate constant of $3.7 \pm 1 \mathrm{x}$ $10^{-11} \mathrm{~cm}^{3} \mathrm{~s}^{-1}(95 \% \mathrm{CL})$. At any given pressure, the Reaction D is approximately 50 times slower than the bare uranium doubly charged ion and becomes rate limiting for the formation of $\mathrm{UO}_{2}{ }^{2+}$ from $\mathrm{U}^{2+}$. Cornehl et al. note that the rate of Reaction $\mathrm{D}$ is more than one order of magnitude slower than Reaction C, occurring at approximately $4 \%$ of the collision rate. ${ }^{31,35}$ If we assume Reaction $\mathrm{C}$ to occur at the collision rate, then Reaction D occurs at approximately $2 \%$ of the collision frequency, in close agreement with the previous reports.

5.3.2.5. $\mathrm{UO}_{2}{ }^{2+}$ reaction with $\mathrm{O}_{2}$

$$
\mathrm{UO}_{2}^{2+}+\mathrm{O}_{2} \rightarrow \mathrm{UO}_{2}^{+}+\mathrm{O}_{2}^{+}
$$

Decreasing the low-mass cut-off value from $110 \mathrm{~m} / \mathrm{z}$ to $25 \mathrm{~m} / \mathrm{z}$ during the reaction period facilitated the observation of $\mathrm{O}_{2}{ }^{+}$, the charge exchange product of Reaction $\mathrm{E}$. However, the reaction rate could not be measured satisfactorily because the reagent $\mathrm{UO}_{2}{ }^{2+}$ could not be obtained in sufficient quantity. This is because Reaction E proceeds at a rate faster than Reaction $\mathrm{D}\left(>3.7 \times 10^{-11} \mathrm{~cm}^{3} \mathrm{~s}^{-1}\right)$ and prevents the accumulation of $\mathrm{UO}_{2}{ }^{2+}$. Resonant ejection of $\mathrm{UO}_{2}{ }^{2+}$ during the reaction period of $\mathrm{UO}^{2+}$ with $\mathrm{O}_{2}$ prevents the formation of both $\mathrm{UO}_{2}{ }^{+}$and $\mathrm{O}_{2}{ }^{+}$, indicating that the alternative charge exchange reaction (not shown in Figure 1) in equation $\mathrm{F}$ is not an energetically feasible pathway.

$$
\mathrm{UO}^{2+}+\mathrm{O}_{2} \longrightarrow \mathrm{X} \rightarrow \mathrm{UO}^{+}+\mathrm{O}_{2}^{+}
$$


This indicates that the ionization potential of $\mathrm{UO}^{+}$is less than that of $\mathrm{O}_{2}(12.06$ $\mathrm{eV})$. Also, given that $\Delta \mathrm{H}_{\mathrm{f}}\left(\mathrm{O}_{2}^{+}\right)=1165 \mathrm{~kJ} \mathrm{~mol}^{-1}$ and $\Delta \mathrm{H}_{\mathrm{f}}\left(\mathrm{UO}^{+}\right)=582 \pm 13 \mathrm{~kJ} \mathrm{~mol}^{-1}$, the enthalpy of formation of $\mathrm{UO}^{2+}$ must be less than or equal to $1747 \mathrm{~kJ} \mathrm{~mol}^{-1}$. This value is considerably smaller than the previous upper limits of $2028 \mathrm{~kJ} \mathrm{~mol}^{-1}$ obtained in section 5.3.2.3., and $2047 \mathrm{~kJ} \mathrm{~mol}^{1}$ determined by Cornehl et al. ${ }^{31}$

\subsubsection{Reactions with $\mathrm{H}_{2} \mathrm{O}$}

The reactions which occur between uranium-containing ions and water are summarized in Figure 6. Isotopically enriched $\mathrm{H}_{2}^{18} \mathrm{O}$ was used in order to distinguish between the reactions with added $\mathrm{H}_{2}{ }^{18} \mathrm{O}$ and those with residual $\mathrm{H}_{2}{ }^{16} \mathrm{O}$ and ${ }^{16} \mathrm{O}_{2}$.

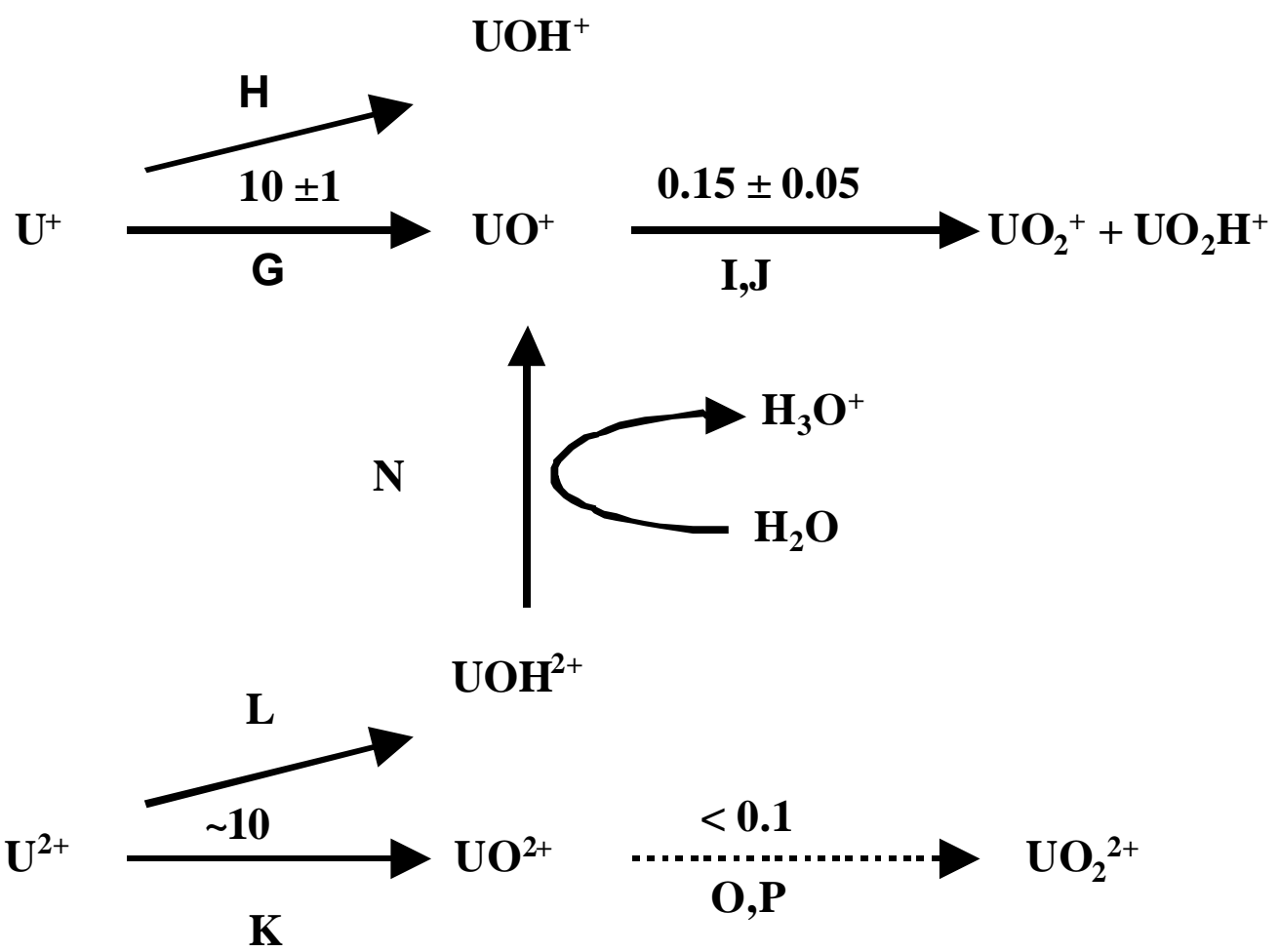

Fig. 5.6. Reaction pathways for the reactions of $\mathrm{U}^{2+}$ and $\mathrm{U}^{+}$with gaseous $\mathrm{H}_{2} \mathrm{O}$. Rates shown have units of $10^{-10} \mathrm{~cm}^{3} \mathrm{~s}^{-1}$. 


\subsubsection{1. $\mathrm{U}^{+}$reaction with $\mathrm{H}_{2} \mathrm{O}$}

$$
\begin{aligned}
& \mathrm{U}^{+}+\mathrm{H}_{2} \mathrm{O} \rightarrow \mathrm{UO}^{+}+\mathrm{H}_{2} \\
& \mathrm{U}^{+}+\mathrm{H}_{2} \mathrm{O} \rightarrow \mathrm{UOH}^{+}+\mathrm{H}
\end{aligned}
$$

There seems to be some disagreement in the literature regarding the relative rates for Reactions $\mathrm{G}$ and $\mathrm{H}$. In low-energy ion beam experiments, both reactions were shown to be exothermic with a branching ratio of approximately 10:1 in favor of Reaction G. ${ }^{32}$ In ICR experiments, Reaction $\mathrm{H}$ was not observed when the bare uranium ions were sufficiently cooled prior to the reaction. ${ }^{35}$ The minor product $\mathrm{UOH}^{+}$was observed in this study with a branching ration close to 10:1, in agreement with the ion beam experiments. Armentrout and Beauchamp ${ }^{32}$ propose a reaction mechanism in which the products of reactions $\mathrm{G}$ and $\mathrm{H}$ are formed from a similar reactive intermediate, $\mathrm{HU}-\mathrm{OH}^{+}$. They show that although both products are formed at the lowest energies possible, the formation of $\mathrm{UO}^{+}$is the thermodynamically favored product at low kinetic energies and that the $\mathrm{UOH}^{+}$ product channel competes more at higher kinetic energies. The product ratio of $\sim 10: 1$ observed here agrees with the lowest energy observations made by Armentrout and Beauchamp, ${ }^{32}$ again indicating that the uranium ions are close to room temperature. The difference in reaction products between the ICR experiments and the ion beam/ion trap experiments is thought to be related to the potential for three-body or third-body collisions. In both cases a third body is required to carry away the energy created by forming the first UO bond and thus prevents the dehydrogenation reaction.

The total rate of loss of $\mathrm{U}^{+}$due to reaction with $\mathrm{H}_{2} \mathrm{O}$ is $1.0 \pm 1 \times 10^{-9} \mathrm{~cm}^{3} \mathrm{~s}^{-1}$,

slightly faster than the reaction with $\mathrm{O}_{2}$ (Reaction A). Armentrout and Beauchamp ${ }^{32}$ also found the cross section for this reaction to be larger than the cross section for the reaction 
with $\mathrm{O}_{2}$. Cornehl et al. ${ }^{35}$ found the reaction rate constant with water to be $0.47 k_{A D O}$ $\pm 40 \%$, which equates to $k=8.1 \times 10^{-10} \mathrm{~cm}^{3} \mathrm{~s}^{-1}$, also faster than the reaction rate with $\mathrm{O}_{2}$. (This calculation was based on the polarizability and dipole values from reference 28 and a "locking factor" of 0.25 obtained from $\mathrm{Su}$ and Bowers ${ }^{33}$ ). Sufficient quantities of $\mathrm{UOH}^{+}$ could not be isolated in order to study the reactions between $\mathrm{UOH}^{+}$and $\mathrm{H}_{2} \mathrm{O}$.

\subsubsection{2. $\mathrm{UO}^{+}$reaction with $\mathrm{H}_{2} \mathrm{O}$}

Reactions I and J have been shown to be exothermic processes. ${ }^{32}$

$$
\begin{aligned}
& \mathrm{UO}^{+}+\mathrm{H}_{2} \mathrm{O} \rightarrow \mathrm{UO}_{2}^{+}+\mathrm{H}_{2} \\
& \mathrm{UO}^{+}+\mathrm{H}_{2} \mathrm{O} \rightarrow \mathrm{UO}_{2} \mathrm{H}^{+}+\mathrm{H}
\end{aligned}
$$

In the present work, the reaction between $\mathrm{UO}^{+}$and water was found to proceed at a rate of $1.5 \pm 1 \times 10^{-11} \mathrm{~cm}^{3} \mathrm{~s}^{-1}$ and showed a branching ratio of approximately $1: 1$, in agreement with Armentrout and Beauchamp. ${ }^{32}$ Cornehl and coworkers ${ }^{35}$ state that the formation of $\mathrm{UO}_{2} \mathrm{H}^{+}$was not observed in their studies. Experiments by Armentrout and Beauchamp and those presented here were conducted at much higher partial pressures (and total pressures) than the work of Cornehl et al. Higher pressures would encourage three-body reactions, or subsequent cooling collisions by a third body, that would not be likely at lower pressures. In the absence of a third body it is possible that an initial exothermic step could provide the driving force to eliminate a molecule of $\mathrm{H}_{2}$ from a reaction with water, i.e. reactions $\mathrm{G}$ and $\mathrm{I}$. If a third body is present at a time frame shorter than that available for dehydrogenation, the third body could take away the excess energy and allow a lower energy channel (i.e. the loss of $\mathrm{H}$ ) to be competitive, reactions $\mathrm{H}$ and $\mathrm{J}$. In Armentrout and Beauchamp's experiments, ${ }^{32}$ the third body would have to be another 
reagent molecule (because the collision cell pressure consists only of the reagent gas), but in these experiments the third body is more likely to be a neon bath gas atom.

\subsubsection{3. $\mathrm{U}^{2+}$ reaction with $\mathrm{H}_{2} \mathrm{O}$}

Two possible reactions between $\mathrm{U}^{2+}$ and $\mathrm{H}_{2} \mathrm{O}$ are given below

$$
\begin{aligned}
& \mathrm{U}^{2+}+\mathrm{H}_{2} \mathrm{O} \rightarrow \mathrm{UO}^{2+}+\mathrm{H}_{2} \\
& \mathrm{U}^{2+}+\mathrm{H}_{2} \mathrm{O} \rightarrow \mathrm{UOH}^{2+}+\mathrm{H}
\end{aligned}
$$

Although the charged products of Reactions $\mathrm{K}$ and $\mathrm{L}$ differ in $\mathrm{m} / \mathrm{z}$ by only 0.5 , these ions can be selectively ejected from the QIT to determine the secondary reaction products of each ion. When $\mathrm{UO}^{2+}$ is resonantly ejected, the product mass spectra include the ions $\mathrm{UOH}^{2+}, \mathrm{H}_{3} \mathrm{O}^{+}$and $\mathrm{UO}^{+}$(as well as the starting reagent $\mathrm{U}^{2+}$ ). No $\mathrm{UOH}^{+}$or $\mathrm{H}_{2} \mathrm{O}^{+}$is

observed, indicating that charge transfer between $\mathrm{UOH}^{2+}$ and $\mathrm{H}_{2} \mathrm{O}$ (Reaction $\mathrm{M}$ ) does not occur.

$$
\mathrm{UOH}^{2+}+\mathrm{H}_{2} \mathrm{O} \longrightarrow \mathrm{X} \rightarrow \mathrm{UOH}^{+}+\mathrm{H}_{2} \mathrm{O}^{+}
$$

Detection of the products $\mathrm{UO}^{+}$and $\mathrm{H}_{3} \mathrm{O}^{+}$indicate that the reaction proceeds via the protonation of a water molecule in the reaction

$$
\mathrm{UOH}^{2+}+\mathrm{H}_{2} \mathrm{O} \rightarrow \mathrm{UO}^{+}+\mathrm{H}_{3} \mathrm{O}^{+}
$$

Thus, while it is not possible to form $\mathrm{UO}^{+}$from a reaction between $\mathrm{U}^{2+}$ and $\mathrm{O}_{2}$, it is possible to form $\mathrm{UO}^{+}$from $\mathrm{U}^{2+}$ via the reaction with water, as demonstrated in the reaction sequence $\mathrm{L}$ and $\mathrm{N}$. Because Reaction $\mathrm{L}$ is exothermic, $\Delta \mathrm{H}_{\mathrm{f}}(\mathrm{H})=218 \mathrm{~kJ} \mathrm{~mol}^{1}$, and $\Delta \mathrm{H}_{\mathrm{f}}\left(\mathrm{H}_{2} \mathrm{O}\right)=-241 \mathrm{~kJ} \mathrm{~mol}^{-1},{ }^{28}$ an upper limit of $1818 \pm 50 \mathrm{~kJ} \mathrm{~mol}^{-1}$ is obtained for $\Delta \mathrm{H}_{\mathrm{f}}$ $\left(\mathrm{UOH}^{2+}\right)$. Likewise, given $\Delta \mathrm{H}_{\mathrm{f}}\left(\mathrm{H}_{3} \mathrm{O}^{+}\right)=592 \mathrm{~kJ} \mathrm{~mol}^{-1}$ and that Reaction $\mathrm{N}$ is also exothermic, a limit is also obtained for $\Delta \mathrm{H}_{\mathrm{f}}\left(\mathrm{UOH}^{2+}\right) \geq 1410 \pm 13 \mathrm{~kJ} \mathrm{~mol}^{-1}$. This 
bracketing gives limits for the enthalpy of formation of $\mathrm{UOH}^{2+}$ of $1410 \leq \Delta \mathrm{H}_{\mathrm{f}}\left(\mathrm{UOH}^{2+}\right) \leq$ $1818 \mathrm{~kJ} \mathrm{~mol}^{1}$.

Reliable reaction rate constants could not be determined for the doubly charged uranium ions because significant ion losses were observed during the course of the reactions. These losses could be due to an unidentified reaction loss path but are more likely due to scattering of the lighter ions that are formed during the reactions (i.e. $\mathrm{H}_{3}{ }^{18} \mathrm{O}^{+}$). If all the $\mathrm{U}^{2+}$ losses do indeed pass quantitatively through $\mathrm{UO}^{2+}$ or $\mathrm{UOH}^{2+}$, the reaction rate constant for the combined Reactions $\mathrm{K}$ and $\mathrm{L}$ would be $1.0 \times 10^{-9} \mathrm{~cm}^{3} \mathrm{~s}^{-1}$. This equates to approximately $0.4 k_{A D O}$. Because $\mathrm{H}_{3}{ }^{18} \mathrm{O}^{+}$was observed at low trapping potentials and the loss rate of $\mathrm{UOH}^{2+}$ was faster than the loss rate of $\mathrm{UO}^{2+}$, the loss of charge is probably due to the fast protonation of water in the form of $\mathrm{H}_{3}{ }^{18} \mathrm{O}^{+}$.

\subsubsection{4. $\mathrm{UO}^{2+}$ reaction with $\mathrm{H}_{2} \mathrm{O}$}

Under the conditions of these experiments, Reactions $\mathrm{O}$ and $\mathrm{P}$ were not observed.

$$
\begin{gathered}
\mathrm{UO}^{2+}+\mathrm{H}_{2} \mathrm{O} \rightarrow \mathrm{UO}_{2}{ }^{2+}+\mathrm{H}_{2} \\
\mathrm{UO}^{2+}+\mathrm{H}_{2} \mathrm{O} \rightarrow \mathrm{UO}^{+}+\mathrm{H}_{2} \mathrm{O}^{+}
\end{gathered}
$$

It is not established if these reactions are endothermic, or just too slow to be

measured. Because the slowest measurable rate on this system is approximately $1 \times 10^{-11}$ $\mathrm{cm}^{3} \mathrm{sec}^{-1}$, we provide this value as an upper limit for reactions $\mathrm{O}$ and $\mathrm{P}$. 


\subsection{Conclusions}

This work describes a methodology for measuring reaction rate constants in a quadrupole ion trap using neon as the bath gas. Kinetic energy effects are considered to be very small—contributing less than $5 \mathrm{~kJ} \mathrm{~mol}^{-1}$ to endothermic reactions-based on ion trap simulations and theoretical considerations. In the simulations, neon brings the ion's kinetic energy to a steady state value more quickly than does helium and this reduces the necessary cooling period before making a rate measurement. The reaction rates and products observed between uranium ions and $\mathrm{H}_{2}^{18} \mathrm{O}$ agree with work conducted in low energy ion beam experiments, but disagree, in select cases, with similar reactions observed in an ICR. These differences are likely to be the result of third body collisions in the QIT and ion beam experiments that help take away excess energy from the reaction intermediates.

The use of a neon bath gas is proving to be extremely useful for advanced

applications of ion traps, including dissociating strongly bound oxide ions ${ }^{18-20}$ and for measuring the thermodynamics and kinetics of reactions. It is hoped that these techniques will afford a promising approach to studying transuranic ions and compounds, where the QIT lends itself particularly well to radiation containment controls. Benefits would include size (for glovebox applications), cost, and the ability to measure reaction rates, thermochemistry, and bond dissociation energies (from CID rates) in a single instrument. 


\subsection{References}

(1) Berkel, G. J. V.; Glish, G. L.; McLuckey, S. A. Anal. Chem. 1990, 62, 1284-1295.

(2) McLuckey, S. A.; Goeringer, D. E. J. Mass Spectrom. 1997, 32, 461-474.

(3) Vedel, F.; Vedel, M.; Brodbelt, J. S. In Practical Aspects of Ion Trap Mass Spectrometry; March, R. E., Todd, J. F. J., Eds.; CRC Press: New York, 1995; Vol. 1, pp 343.

(4) Bonner, R. F.; Lawson, G.; Todd, J. F. J. Int. J. Mass Spectrom. Ion Phys. 1972/73, 10, 197-203.

(5) Liere, P.; Steiner, V.; Jennings, K. R.; March, R. E.; Tabet, J. C. Int. J. Mass Spectrom. Ion Preocesses. 1997, 167/168, 735-751.

(6) Armentrout, P. B.; Kickel, B. L. In Organometallic Ion Chemistry; Freiser, B. S., Ed.; Kluwer: Norwell, MA, 1996; Vol. 15.

(7) Lawson, G.; Bonner, R. F.; Mather, R. E.; Todd, J. F. J.; March, R. E. J. Chem. Soc. Faraday Trans. 1976, 72, 545-557.

(8) Fulford, J. E.; Dupuis, J. W.; March, R. E. Can. J. Chem. 1978, 56, 2324-2330.

(9) Dawson, P. H. Int. J. Mass Spectrom. Ion Phys. 1976, 20, 237-245.

(10) Armitage, M. A.; Higgins, M. J.; Lewars, E. G.; March, R. E. J. Am. Chem. Soc. 1980, 102, 5064--5068.

(11) Cutler, L. S.; Flory, C. A.; Giffard, R. P.; McGuire, M. D. Apll. Phys. B 1986, 39, 251-259.

(12) Schaaf, H.; Schmeling, U.; Werth, G. Appl. Phys. 1981, 25, 249-251. 
(13) Siemers, I.; Blatt, R.; Sauter, T.; Neuhauser, W. Phys. Rev. A. 1988, 38, 51215128.

(14) Vedel, F. Int. J. Mass Spectrom. Ion Processes 1991, 106, 33-61.

(15) Brodbelt-Lustig, J. S.; Cooks, R. G. Talanta 1989, 36, 255-260.

(16) Lovejoy, E. R.; Wilson, R. R. J. Phys. Chem. A. 1998, 102, 2309-2315.

(17) Nourse, B. D.; Kenttamaa, H. I. J. Phys. Chem. 1990, 94, 5809-5812.

(18) Duckworth, D. C.; McLuckey, S. A., 26th Federation of Analytical Chemistry and Spectroscopy Societies Conference, Vancouver, Canada, October 1999.

(19) Duckworth, D. C.; Goeringer, D. E.; McLuckey, S. A. J. Am. Soc. Mass Spectrom. 2000, 11, 1072-1078.

(20) Jackson, G. P.; King, F. L.; Goeringer, D. E.; Duckworth, D. C. Int. J. Mass Spectrom. 2002, 216, 85-93.

(21) Goeringer, D. E.; Asano, K. G.; McLuckey, S. A. Int. J. Mass Spectrom. 1999, 182/183, 275-288.

(22) Duckworth, D. C.; Marcus, R. K. J. Anal. At. Mass Spectrom. 1992, 7, 711-715.

(23) Bui, H. A.; Cooks, R. G. J. Mass Spectrom. 1998, 33, 297-304.

(24) Viehland; Mason J. Chem. Phys. 1977, 66, 422-434.

(25) Wannier, G. H. Bell Syst. Tech. J. 1953, 120, 32.

(26) Johnsen, R.; Biondi, M. A. J. Chem. Phys. 1972, 57, 1975-1979.

(27) Gieray, R. A.; Reilly, P. T. A.; Yang, M.; Whitten, W. B.; Ramsey, J. M. Anal. Chem. 1998, 70, 117-120.

(28) Lide, D. R., Ed. CRC Handbook of Chemistry and Physics., 81 ed.; CRC Press: New York, 2000. 
(29) Lias, S. G.; Liebman, J. F.; Levin, R. D. J. Phys. Chem. Ref. Data 1984, 13, 695808.

(30) Hildenbrand, D. L.; Guvich, L. V.; Yungman, V. S. The Chemical Thermodynamics of the Actinide Elements and Compounds Part 13.; IAEA: Vienna, 1985.

(31) Cornehl, H. H.; Heine mann, C.; Marcalo, J.; PiresdeMatos, A.; Schwarz, H. Angew. Chem. Int. Ed. Engl. 1996, 35, 891-894.

(32) Armentrout, P. B.; Beauchamp, J. L. Chem. Phys. 1980, 50, 27-36.

(33) Su, T.; Bowers, M. T. Int. J. Mass Spectrom. Ion Physics 1973, 12, 347-356.

(34) Atkins, P. Physical Chemistry, Sixth ed.; W. H. Freeman and Company: New York, 1998.

(35) Cornehl, H. H.; Wesendrup, R.; Diefenbach, M.; Schwarz, H. Chem. Eur. J. 1997, 3, 1083-1090. 


\section{Chapter 6}

\section{Collision-Induced Dissociation of Lanthanide Oxide Ions in Quadrupole Ion Traps: Effects of Bond Strength and Mass}

\subsection{Introduction}

Recent studies in our laboratory have focused on the measurement of collisioninduced dissociation (CID) rates of strongly bound metal oxide ions such as $\mathrm{TaO}^{+}\left(\mathrm{D}_{0}=\right.$ $790 \mathrm{~kJ} \mathrm{~mol}^{1}$ ) stored in quadrupole ion traps (QIT's). ${ }^{1}$ The purpose of this work is to provide an experimental basis for extending the understanding and use of a thermal reaction model $^{2-5}$ of CID in ion trap mass spectrometers to diatomic ions. A potential benefit is the capability of determining relative and absolute dissociation energies via CID measurements in QIT instruments. Furthermore, the complete dissociation of metal oxide ions is useful in elemental determinations where isobaric spectral interferences occur. $^{6-9}$ The use of CID in ion traps could serve as an alternative and universal approach to overcoming chemical interferences.

There currently exist several methods for determining dissociation energies using CID in quadrupole ion traps. Hart and McLuckey ${ }^{10}$ found a logarithmic dependence of the critical energy for decomposition on the threshold excitation voltage amplitude. The threshold excitation voltage is defined as the minimum voltage required to dissociate a polyatomic ion. Extrapolating a plot of dissociation rate versus excitation amplitude to zero rate yields the threshold excitation voltage. Using polyatomic ions of low dissociation energy $\left(<300 \mathrm{~kJ} \mathrm{~mol}^{-1}\right)$, a calibration curve was obtained for use in the 
determination of an 'unknown' dissociation energy. Shifts in the resonance absorption frequency complicate these experiments, especially at higher dissociation energies $(>200$ $\mathrm{kJ} \mathrm{mol}^{1}$ ) where higher-order fields come in to play with increasing excitation time and amplitude. Additionally, space charge effects cause the fundamental secular frequency of an ion cloud to shift as a function of ion density. ${ }^{11}$ These factors make it very difficult (impossible using some software packages) to maintain on-resonance conditions during an experiment.

Colorado and Brodbelt proposed an alternative method for measuring bond dissociation energies. ${ }^{12}$ In their approach, an on-resonance, low-amplitude excitation voltage is applied to the endcaps for a fixed time. The degree of fragmentation is then measured. The excitation voltage is increased, incrementally, until a significant degree $(>50 \%)$ of fragmentation occurs. The threshold excitation voltage in this case is defined as the excitation amplitude required to yield $10 \%$ dissociation of the molecular ion. In this case, a plot of the threshold excitation voltage versus threshold fragmentation energies gives a linear dependence, instead of the logarithmic dependence observed by Hart and McLuckey. Separate calibration curves are required when observing different systems (e.g., hydrogen-bonded complexes versus covalently bound molecules), although the reasons are not completely clear at this time. The experiments conducted by Hart and McLuckey, and Colorado and Brodbelt were optimized for polyatomic (organic) molecules having threshold energies for fragmentation $\leq 300 \mathrm{~kJ} \mathrm{~mol}^{-1}$. The metal oxides of interest in this study, however, have dissociation energies on the order of $550-850 \mathrm{~kJ}$ $\mathrm{mol}^{-1}$. Under the conditions used by the above authors, the oxide ions in this study would be scattered from the trap long before reaching the required voltage for dissociation. The 
low trapping potential $\left(\mathrm{q}_{\mathrm{z}}<0.4\right)$ and the modest center-of-mass collision energy associated with a helium bath gas do not impart enough energy into the ions to cause dissociation. Therefore, special conditions are required to achieve dissociation of these strongly bound diatomic species.

Duckworth et al. showed that by using a $\mathrm{q}_{\mathrm{z}}$ of 0.67 and $\sim 0.5$ mTorr neon $(1$ Torr $=$ $133.3 \mathrm{~Pa}$ ) as the bath gas, dissociation yields close to $100 \%$ could be obtained for $\mathrm{TaO}^{+}$ within 20-50 ms of excitation. ${ }^{1}$ The large potential well depth associated with such a $\mathrm{q}_{\mathrm{z}}$ value allows higher amplitude excitation voltages to be applied ${ }^{13}$ and greater kinetic energies to be imparted to the ions. The neon collision target more efficiently converts the kinetic energy to internal energy than helium. ${ }^{14}$ Goeringer et al. simulated the ion trap CID kinetics of $\mathrm{TaO}^{+}$by applying a thermal unimolecular reaction model. ${ }^{5}$ An internal temperature for $\mathrm{TaO}^{+}$on the order of $15000 \mathrm{~K}$ was predicted for the conditions used.

For measured dissociation rates to be useful in the determination of dissociation energies, it is necessary to compare them without the complication of mass effects. This work provides new insight into making reliable CID rate measurements and presents a new approach to determining dissociation rates that ensures that on-resonance rate measurement conditions are established. Determination relative to an internal standard accounts for temporal fluctuations in CID response. An empirical correction for mass effects on CID rates is determined and applied to measured CID rates. Corrected dissociation rates are used to quantify bond dissociation energies of lanthanide monoxide ions. 


\subsection{Experimental}

A pulsed direct current glow discharge (GD) ionization source was coupled to a Teledyne 3DQ quadrupole ion trap mass spectrometer, as described previously. ${ }^{4}$ Metal oxides of interest were mixed in a $\sim 1: 1$ ratio with praseodymium oxide (the internal standard) and pressed onto the surface of a gold or indium pin. Samples were sputtered in the neon discharge (0.8 Torr) until a steady state signal was observed. Metal and metal oxide ions were trapped and allowed to react with molecular oxygen that was admitted into the trap at a pressure of $8 \times 10^{-7}$ Torr. Neon made up the remaining bath gas pressure to a total of $5 \times 10^{-4}$ Torr.

A typical scan function involved the following:

1) $5 \mathrm{~ms}$ metal ion accumulation period, with a single frequency excitation signal applied to resonantly eject the matrix ions (m/z 197 for gold pins, m/z 115 for indium).

2) 50-100 ms reaction period during which metal ions were allowed to react with molecular oxygen at $\mathrm{q}_{\mathrm{z}}=0.2$.

3) $5 \mathrm{~ms}$ oxide ion isolation period using mass selective instability ${ }^{15}$ and filtered noise fields (FNF) ${ }^{16}$ to obtain the ion of interest.

4) $30 \mathrm{~ms}$ ion cooling period at $\mathrm{q}_{\mathrm{z}}=0.2$.

5) $20 \mathrm{~ms}, 226 \mathrm{kHz}, 250 \mathrm{mV}_{\mathrm{pp}}$ resonance excitation signal applied in a dipolar fashion at $\mathrm{q}_{\mathrm{z}} \sim 0.67 .{ }^{17}$ The $\mathrm{rf}$ amplitude was scanned over the absorption range in order to determine the point of maximum dissociation. A second excitation frequency $(\sim 260 \mathrm{kHz})$ was simultaneously applied to eject the product metal ions as they formed thereby preventing the forward oxidation reaction. 
6) $10 \mathrm{~ms}$ cooling period at $\mathrm{q}_{\mathrm{z}}=0.2$ to collisionally cool the ions before mass analysis.

7) Mass analysis from m/z 130-200 at $12000 \mathrm{amu} \mathrm{sec}^{-1}$ using axial modulation (Mass spectra for yttrium oxide were taken from m/z 80-150).

Initial dissociation rate measurements obtained using the absorption curve method described above gave inconsistent results because no attempt was made to isolate a single isotope of a given oxide ion in step three above. It was found that, as has been noted in previous reports, ${ }^{11}$ that the ion number density in the trap greatly affects the degree of molecular ion fragmentation. In the case of lanthanides with several isotopes, the effects of space charge from the non-dissociated isotopes are significant. Considerable improvement in reproducibility was achieved by isolating an individual isotope prior to the dissociation step. However, space charge effects still existed because of differences in number densities of ions having the same nominal mass. It was found that the initial ion number density changed both the resonance frequency and the degree of fragmentation that occurred. The degree of dissociation increased as the initial ion signal decreased because of the decreased coulombic repulsion occurring in the ion cloud at lower ion number densities. When higher number densities exist, a wider distribution of secular frequencies occurs and not all the ions will be in resonance at once. When fewer ions are stored, there is less charge repulsion and a larger fraction of ions will be in resonance and will acquire the energy required to dissociate.

A linear relationship was found between the maximum fractional dissociation and the initial ion number density. Therefore, a correction could be made to normalize the 
fractional dissociation if the initial ion abundance deviated significantly from a desired point. It was preferable, however, to maintain a consistent ion abundance prior to each dissociation experiment so that corrections were not necessary. This was achieved by using an enriched isotope as the sample in the glow discharge. Quantitative isolation was achieved using FNF frequencies to eject any remaining isotopes and product ions. The glow discharge voltage was adjusted to maintain the desired ion number density. Data averaging of 32 scans constituted a single analysis. In all cases, four repeat analyses were made intermittently between the internal standard and the ion of interest.

\subsubsection{Method development}

Previous experiments in this laboratory directly measured the dissociation rate of tantalum oxide ions by monitoring the metal oxide intensity as a function of increasing resonance excitation times. ${ }^{1,5}$ For each rate determination the excitation frequency was set to $226 \mathrm{kHz}$ and the $\mathrm{rf}$ trapping potential amplitude was tuned to produce maximum dissociation at a single resonance excitation time. Tuning the ion into resonance at 226 $\mathrm{kHz}$ by adjusting the rf trapping potential amplitude improves frequency resolution over tuning the resonance frequency (limited to a $1 \mathrm{kHz}$ resolution on this system) at a fixed trapping potential. This also ensures that a constant $\mathrm{q}_{\mathrm{z}}$ and an optimum resonance overlap is achieved.

In initial attempts to apply the same methodology used for $\mathrm{TaO}^{+}$to lanthanide oxide ions, precision and accuracy were degraded because of shifts in resonance excitation frequency. It has been shown that resonance frequency shifts result as a

function of ion abundance, and resonance excitation time and voltage. ${ }^{10,11}$ Therefore, for 
the most accurate rate measurements dynamic tuning of the resonance excitation is required as a function of resonance excitation time, voltage, and ion abundance. For example, the procedure described by Hart and McLuckey required the alteration of both the dissociation time and the tuning frequency during an automated scan function. As this programming ability is not present for our instrument (or for most commercially available instruments), an alternative method for measuring dissociation properties is used that does not require a change of more than one variable in a given scan function.

The use of resonance absorption curves is presented as an alternative to that proposed in references ${ }^{1}$ and ${ }^{10}$ because shifts in resonance frequency are easily observed and corrected. Although resonance absorption curves normally do not provide the detailed kinetic information contained in dissociation rate measurements it is possible to convert the fractional dissociation determined from a resonance absorption curve to a phenomenological rate. Assuming a pseudo-first order dissociation rate, as established in references ${ }^{1}$ and ${ }^{10}$, the abundance of a lanthanide oxide, $\left[\operatorname{LnO}^{+}\right]$, at time $t$ can be given as

$$
\left[\operatorname{LnO}^{+}\right]_{t}=\left[\operatorname{LnO}^{+}\right]_{0} \exp ^{-\left[n k_{t o t} t\right]}
$$

where $\left[\mathrm{LnO}^{+}\right]_{0}$ is the initial ion abundance, $n$ is the collision gas number density, and $k_{t o t}$ is the rate constant for all $\mathrm{LnO}^{+}$losses. The sum of the loss rates, $n k_{t o t}$, is given by

$$
n k_{\text {tot }}=n k_{\text {diss }}+n k_{\text {scatt }}+n k_{\text {react }}
$$

and if the scattering rate, $n k_{\text {scatt }}$, and alternative reaction rate, $n k_{\text {react }}$, can be shown to be negligible, $n k_{t o t}$ will approximate to the dissociation rate $n k_{\text {diss }}$. This condition was established using conditions wherein $\sim 100 \%$ of the product metal ions were recovered. ${ }^{1}$ 
The fractional dissociation, determined from the absorption curve at a fixed dissociation time $t$, can be converted to a dissociation rate according to the equation

$$
n k_{\text {diss }}=\frac{-\ln \left(\frac{\left[\operatorname{LnO} O^{+}\right]_{t}}{\left[\operatorname{LnO} O^{+}\right]_{0}}\right)}{t}
$$

where $n k_{d i s s}$ is the phenomenological rate for a given bath gas pressure and excitation amplitude. Measuring the rate at the point of maximum dissociation in the absorption curves alleviates the problems associated with resonance shifts. In addition, the dissociation measurement is not dependent on the arbitrary dissociation time used to collect the resonance absorption curve, but is dependent only on the excitation amplitude. This allows modest extension of the method as needed due to differences in rate due to mass and dissociation energy.

\subsection{Results and discussion}

The experimental approach employed in earlier work for $\mathrm{TaO}^{+}$was applied to the oxide ion of lanthanum $\left(\mathrm{D}_{0}=860 \mathrm{~kJ} \mathrm{~mol}^{-1}\right){ }^{1}$ Figure 6.1 shows the phenomenological dissociation rates for lanthanum and tantalum oxide ions as a function of resonance excitation amplitude. Interestingly, the lanthanum oxide ion was found to dissociate at much lower excitation voltages than the tantalum oxide ion $\left(D_{0}=790 \mathrm{~kJ}\right.$ $\mathrm{mol}^{-1}$ ). Thus a series of CID experiments were performed on a suite of lanthanide (plus yttrium) monoxide ions to determine the role of bond dissociation energy and ion mass on measured dissociation rates. 


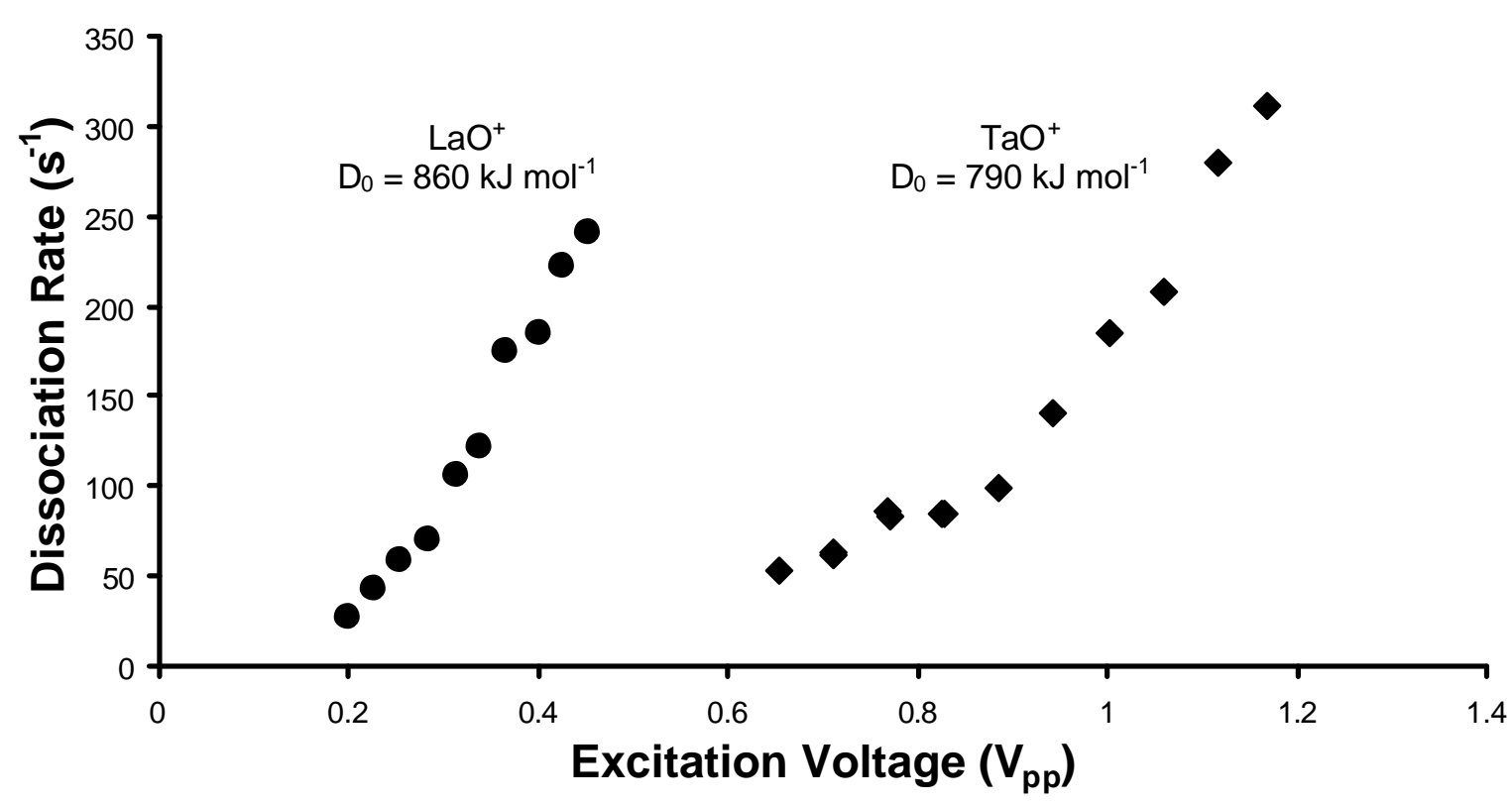

Fig. 6.1. Comparison of dissociation rates as a function of resonance excitation voltage for $\mathrm{LaO}^{+}$and $\mathrm{TaO}^{+}$. Secular frequency $=226 \mathrm{kHz}\left(\mathrm{q}_{\mathrm{z}}-0.67\right), 0.5 \mathrm{mTorr} \mathrm{Ne}$ collision gas.

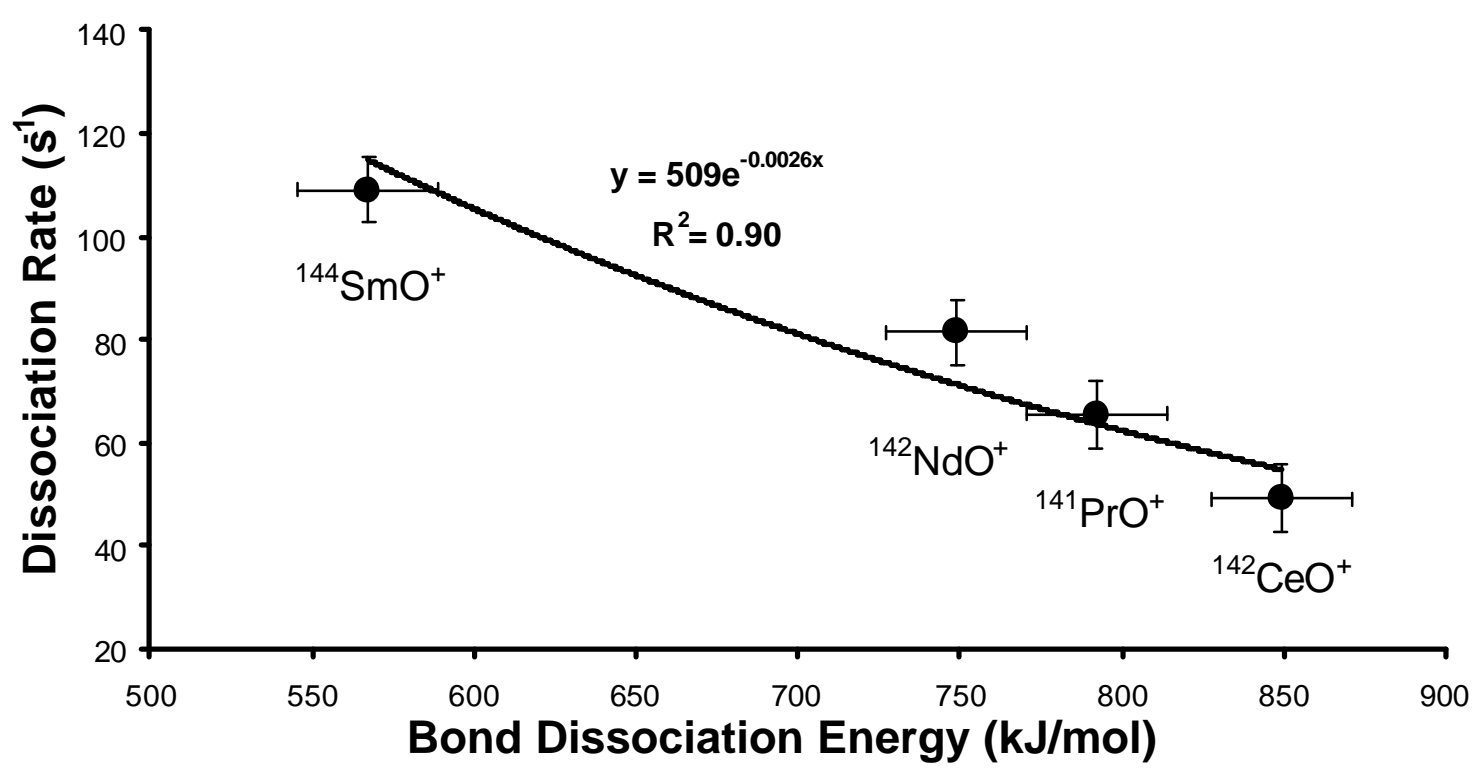

Fig. 6.2. Plot of the dissociation rates as a function of bond dissociation energy for oxide ions having the same nominal mass to determine the sensitivity of the measurement to dissociation energy. Reprinted from G.P. Jackson et al. Int. J. Mass Spectrom. 216 (2002) 85-93. 


\subsubsection{Bond strength effects on dissociation rate}

To characterize the relationship between bond dissociation energy and CID rate, oxide ions having similar masses (variation $\leq 5 \mathrm{amu}$ ) but a wide range of dissociation energies were examined. Figure 6.2 shows the dissociation rate versus bond strength for a number of oxide ions having very similar masses. The rates were calculated using Equation 6.3 and were normalized to the dissociation rate of the internal standard, $\mathrm{PrO}^{+}$. Calculations using a thermal model for the dissociation of diatomic ions having a Boltzmann distribution of internal energies suggest that CID rates decrease exponentially with dissociation energy. ${ }^{5,18}$ Thus, an exponential fit $\left(R^{2}=0.90\right)$ is used in Figure 6.2.

The measured dependence of CID rate on dissociation energy is approximately $0.2 \mathrm{~mol} \mathrm{~kJ}^{-1} \mathrm{~s}^{-1}$ (for mass 157); thus, indicating that the dissociation rate decreases by $1 \mathrm{~s}^{-1}$ for every $5 \mathrm{~kJ} \mathrm{~mol}^{-1}$ increase in dissociation energy. The standard deviations of the dissociation rates obtained in this experiment average $\sim 5 \mathrm{~s}^{-1}(\mathrm{n}=4)$, which is equivalent to a standard deviation of the bond energy measurement of $\sim 25 \mathrm{~kJ} \mathrm{~mol}^{1}$. This error is marginally larger than the internal error usually reported for thermodynamic data of this

kind. ${ }^{19,20}$ As for accuracy, the values calculated here agree with the average of reported values $( \pm 1 \sigma)$.

\subsubsection{Mass effects on dissociation rate}

The effect of mass on the dissociation rates of metal monoxide ions can be determined by measuring the CID rates for each of the metal oxides of yttrium, neodymium, and gadolinium. These oxides have similar bond dissociation energies and are listed in Table 6.1 along with other metals considered in this study. These elements 
(indicated with a '\#' in Table 6.1) were selected on the basis of several characteristics: (1) the dissociation energies have been reasonably well established and lie within a $50 \mathrm{~kJ}$ $\mathrm{mol}^{-1}$ range, (2) the monoxide ion can be formed via metal ion molecule reactions with molecular oxygen under the conditions of the experiment, (3) the monoxide on can be isolated and stored at the designated starting number density, and (4) the monoxide can be dissociated without chemical reaction or scattering losses under the defined CID conditions.

Table 6.1. Dissociation energy and masses of the oxide ions used.

\begin{tabular}{c|c|c} 
Element oxide ion & Oxide ion mass $(\mathrm{amu})$ & $\mathrm{D}_{0}\left(\mathrm{~kJ} \mathrm{~mol}^{-1}\right)$ \\
\hline \hline $\mathrm{YO}^{+}$ & 105 & $725^{\mathrm{a} \#}( \pm 18)$ \\
\hline $\mathrm{CeO}^{+}$ & $158^{*}$ & $849^{\mathrm{b}}( \pm 22)$ \\
\hline $\mathrm{PrO}^{+}$ & $157^{*}$ & $792^{\mathrm{b}}( \pm 22)$ \\
\hline $\mathrm{NdO}^{+}$ & $158^{*}$ & $749^{\mathrm{b} \#}( \pm 22)$ \\
\hline $\mathrm{NdO}^{+}$ & 162 & $749^{\mathrm{b} \#}( \pm 22)$ \\
\hline $\mathrm{SmO}^{+}$ & $160^{*}$ & $567^{\mathrm{b}}( \pm 22)$ \\
\hline $\mathrm{GdO}^{+}$ & 170 & $735^{\mathrm{b \#}}( \pm 22)$
\end{tabular}

* used to characterize the sensitivity to bond strength for near constant mass.

\# used to characterize the sensitivity to mass at near constant bond strength.

${ }^{a}$ see references ${ }^{20}$ and ${ }^{21}$.

${ }^{\mathrm{b}}$ see reference ${ }^{19}$ and references therein. 
The fractional dissociations of ${ }^{89} \mathrm{YO}^{+},{ }^{142 \& 146} \mathrm{NdO}^{+}$, and ${ }^{154} \mathrm{GdO}^{+}$were normalized to the internal standard $\left(\mathrm{PrO}^{+}\right)$, converted to a dissociation rate using Equation 6.3, and plotted versus monoxide ion mass in Figure 6.3. Unfortunately, no monoxide ion could be found of similar bond strength and intermediate mass $\left(105<\mathrm{LnO}^{+}<158\right)$ thereby limiting the number of data points in this plot. The line of best fit $\left(\mathrm{R}^{2}=0.995\right)$ gives a mass correction of $-1.15 \mathrm{~s}^{-1} \mathrm{amu}^{-1}$. Also, no other suite of metal monoxides could be found having different bond dissociation energy that also meets the above criteria and spanned such a wide mass range. Thus this sensitivity of the dissociation rate to mass has only been established for a single bond dissociation energy of $735 \mathrm{~kJ} \mathrm{~mol}^{-1}$. Evidence will be provided later showing that the CID rate dependence on monoxide ion mass is constant over the range of dissociation energies and oxide masses considered in this report.

Table 6.2. Dissociation energy determinations of 'unknowns' using the empirical and theoretical correction terms for mass effects.

\begin{tabular}{c|c|c|} 
Element oxide ion & Literature $\mathrm{D}_{0}\left(\mathrm{~kJ} \mathrm{~mol}^{1}\right)$ & Measured $\mathrm{D}_{0}\left(\mathrm{~kJ} \mathrm{~mol}^{-1}\right)$ \\
\hline \hline $\mathrm{YO}^{+}$ & $725^{\mathrm{a}}( \pm 18)$ & $718( \pm 25)$ \\
\hline $\mathrm{LaO}^{+}$ & $849^{\mathrm{b}}( \pm 22)$ & $875( \pm 25)$ \\
\hline $\mathrm{GdO}^{+}$ & $735^{\mathrm{b}}( \pm 22)$ & $524( \pm 25)$ \\
\hline $\mathrm{HoO}^{+}$ & $597^{\mathrm{b}}( \pm 22)$ & \\
${ }^{\mathrm{a}}$ see references & \\
& &
\end{tabular}




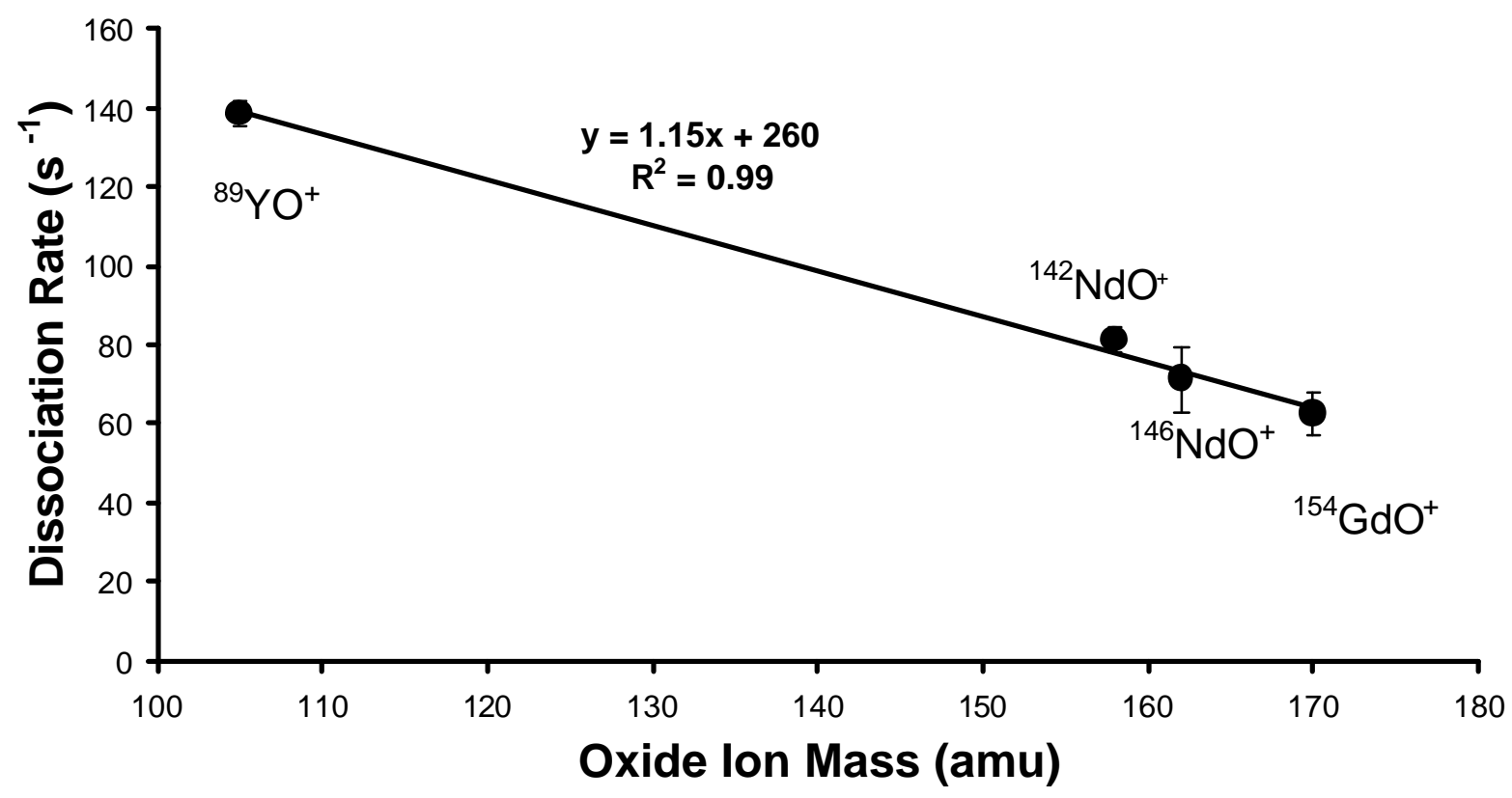

Fig. 6.3. Plot of the dissociation rate versus oxide ion mass for compounds having the same nominal dissociation energy to determine the effect of mass on the dissociation rate. Reprinted from G.P. Jackson et al. Int. J. Mass Spectrom. 216 (2002) 85-93.

\subsubsection{Determining dissociation energy of 'unknowns'}

Using the correction term for mass from Figure 6.3 and the bond energy calibration of Figure 6.2, it is possible to estimate the dissociation energy of a diatomic oxide ion from its dissociation rate and mass. Each of the oxide ions listed in Table 6.2 was dissociated under the conditions described above. These oxides were not included in the calibration curves and represent a range of bond energies and oxide ion masses. The dissociation rate was calculated from the corresponding fractional dissociation values following normalization to the value for praseodymium using Equation 6.3. Finally, the 
equations for the curves in Figures 6.2 and 6.3 were used to convert the rates to dissociation energies. The calculated dissociation energies are presented in Table 6.2. There is good agreement between the experimental results and those found in the literature. It should be noted that this method provides an estimate of bond dissociation energy with an internal precision that is equivalent to the inter-laboratory precision calculated from literature values.

\subsubsection{Bond strength and mass effects}

Further evidence of the validity of the mass correction over the mass range of the lanthanide (plus yttrium) oxides and the correlation of measured rates to bond dissociation energy can be seen when the corrected and uncorrected CID rates are compared for the suite of elements. The dissociation rates for all the lanthanide oxide ions in Tables 6.1 and 6.2 (normalized to that for $\mathrm{PrO}^{+}$) are shown in Figure 6.4.a. This plot shows that without taking into account the influence of mass on the rate measurement, there is very little correlation between dissociation rate and bond strength $\left(\mathrm{R}^{2}=0.51\right)$. Some correlation exists because of the limited mass range of the lanthanide elements. Because the mass of each ion and its affect on dissociation rate is known, it is possible to adjust the measured rates using the empirical correction

$$
n k_{\text {corr }}=n k_{\text {meas }} *\left(1+1.15\left(M_{L^{n O^{+}}}-M_{P r O^{+}}\right)\right)
$$

where $n k_{\text {corr }}$ is the empirically corrected dissociation rate and $n k_{\text {meas }}$ is the measured dissociation rate. Figure 6.4.b is a plot of the rates from Figure 6.4.a that have been adjusted using Equation 6.4. It is clear that once the mass effects are removed there is a strong relationship between the dissociation rate and bond strength $\left(R^{2}=0.94\right)$, 
making it possible to construct a calibration curve of different bond dissociation energies that can be used in the determination of such values for an unknown. 

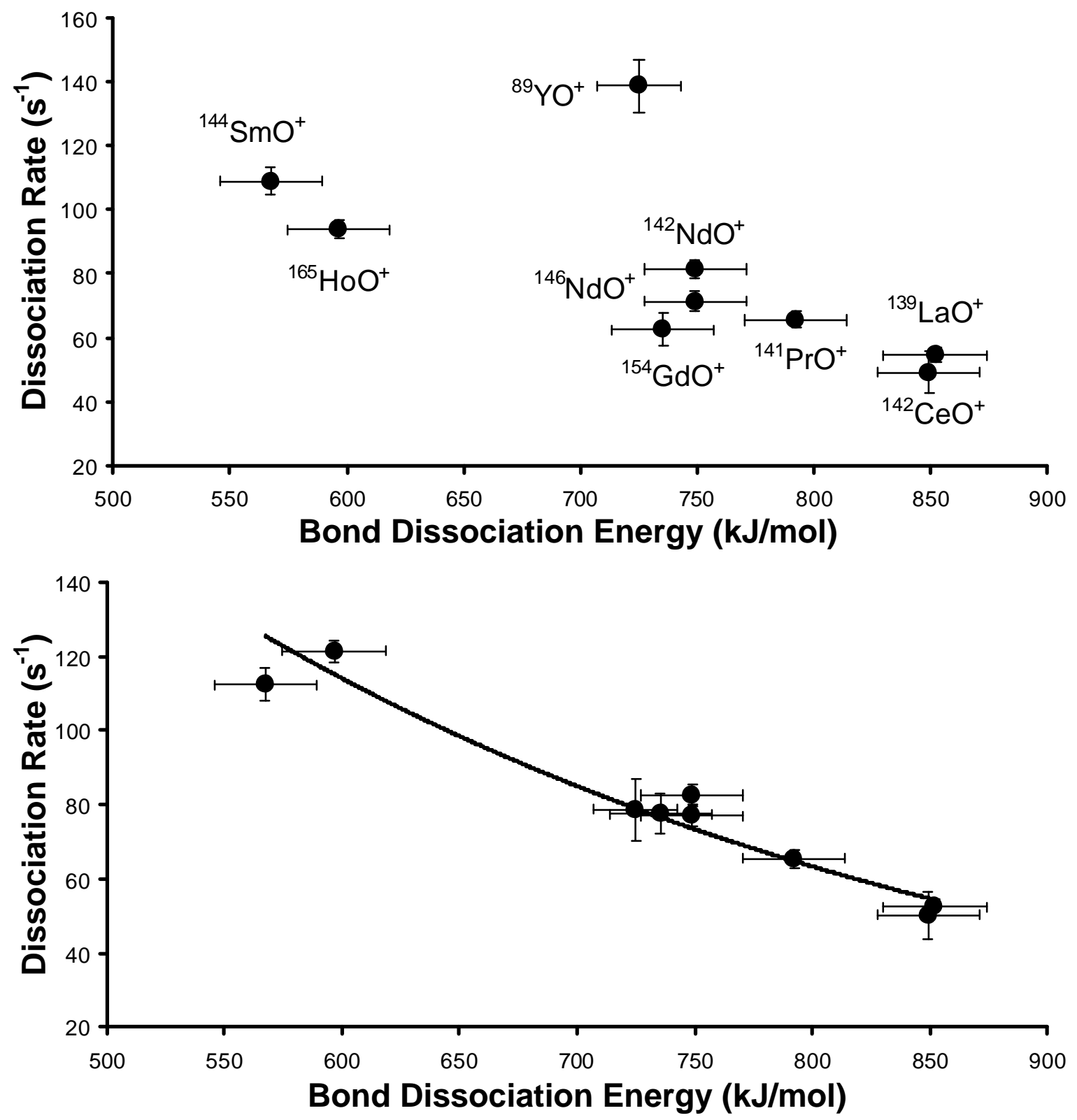

Figure 4. Plot of dissociation rates as a function of bond dissociation a) before and b) after correction for mass effects. Reprinted from G.P. Jackson et al. Int. J. Mass Spectrom. 216 (2002) 85-93. 


\subsection{Conclusions}

The method used in this project to make dissociation rate measurements is sensitive to the mass of the dissociating ion. The sensitivity of the rate on mass can be empirically corrected for, by producing a calibration curve of compounds having the same bond strength but different masses. The slope of this curve can be used to account for the influence of mass on CID rate. Mass-corrected CID rates can then be used to provide a relationship between bond strength and dissociation rate. Over the range of masses and voltages studied, it was also possible to obtain an estimate for $D_{0}$ values based on such corrections.

The dynamic range of the calibration curve is currently limited to the oxide ions that dissociate at similar excitation voltages as the internal standard, praseodymium oxide. Although we are able to dissociate high mass and strongly bound diatomic oxide ions (e.g., $\mathrm{UO}^{+}$) with unit efficiency, the voltage required to effect a detectable dissociation rate falls beyond the resonance excitation voltage used for our calibration curve. Thus, the effect of voltage on dissociation rate measurements will be an important consideration in extending the working range of this method.

Ongoing investigations focus on refining the thermal reaction model for CID to take into account the effect of mass on dissociation rate with the goal of refining the predictive capabilities of the model in light of new empirical evidence. Future work will also consider other diatomic systems such as metal sulfides to test the applicability and universality of the mass and bond energy calibrations. Application of this technique to small polyatomic ions such as metal methoxides and hydroxides will extend our understanding and the utility of this approach to polyatomic systems. Understanding both 
mass and voltage effects will enable a rapid probe for the determination of dissociation / fragmentation energetics—particularly important for organometallic and organic ions. 


\subsection{References}

(1) Duckworth, D. C.; Goeringer, D. E.; McLuckey, S. A. J. Am. Soc. Mass Spectrom. 2000, 11, 1072-1078.

(2) Goeringer, D. E.; McLuckey, S. A. J. Chem. Phys. 1996, 104, 2214-2221.

(3) Goeringer, D. E.; McLuckey, S. A. Rapid Commun. Mass Spectrom. 1996, 100, 328-334.

(4) Goeringer, D. E.; Asano, K. G.; McLuckey, S. A. Int. J. Mass Spectrom. 1999, $182 / 183,275-288$.

(5) Goeringer, D. E.; Duckworth, D. C.; McLuckey, S. A. J. Phys. Chem. A 2001, $105,1882-1889$.

(6) King, F. L.; Harrison, W. W. Int. J. Mass Spectrom. Ion. Processes 1989, 89, 171185.

(7) Duckworth, D. C.; Marcus, R. K. Appl. Spectrosc. 1990, 44, 649-655.

(8) Mei, Y.; Duckworth, D. C.; Cable, P. R.; Marcus, R. K. J. Am. Soc. Mass Spectrom. 1994, 5, 845-851.

(9) Rowan, J. T.; Houk, R. S. Appl. Spectrosc. 1989, 43, 976-980.

(10) Hart, K. J.; McLuckey, S. A. J. Am. Soc. Mass Spectrom. 1993, 5, 250-259.

(11) Vedel, F.; Vedel, M.; Brodbelt, J. S. In Practical Aspects of Ion Trap Mass Spectrometry; March, R. E., Todd, J. F. J., Eds.; CRC Press: New York, 1995; Vol. 1, pp 343.

(12) Colorado, A.; Brodbelt, J. J. Amer. Soc. Mass Spectrom. 1996, 7, 1116-1125. 
(13) Charles, M. J.; McLuckey, S. A.; Glish, G. L. J. Am. Soc. Mass Spectrom. 1994, $5,1031-1041$.

(14) McLuckey, S. A. J. Am. Soc. Mass Spectrom. 1991, 3, 599-614.

(15) Stafford, G. C.; Kelly, P. E.; Syka, J. E. P.; Reynolds, W. E.; Todd, J. F. J. Int. J. Mass Spectrom. Ion Processes 1984, 60, 85-98.

(16) Goeringer, D. E.; Asano, K. G.; McLuckey, S. A.; Hoekman, D.; Stiller, S. W. Anal. Chem. 1994, 66, 313-318.

(17) Louris, J. N.; Cooks, R. G.; Syka, J. E. P.; Kelly, P. E.; G. C. Stafford, J.; Todd, J. F. J. Anal. Chem. 1987, 59, 1677-1685.

(18) Jackson, G. P.; Duckworth, D. C.; Goeringer, D. E. Unpublished results.

(19) Chandrasekharaiah, M. S.; Gingerich, K. A. In Handbook on the Physics and Chemistry of the Rare Earths; K. A. Gschneidner, J., Eyring, L., Eds.; Elsevier: New York, 1989; Vol. 12, pp 409-430.

(20) Seivers, M. R.; Chen, Y.; Armentrout, P. B. J. Chem. Phys. 1996, 105, 63226333.

(21) Clemmer, D. E.; Dalleska, N. F.; Armentrout, P. B. Chem. Phys. Lett. 1992, 190, 259-265. 


\section{Chapter 7}

\section{$\underline{\text { Removal of Molecular Interferences in Elemental Quadrupole Ion Trap }}$ Mass Spectrometry Using Collision Induced Dissociation}

\subsection{Introduction}

Plasma ion sources for mass spectrometry, such as Inductively Coupled Plasmas (ICP) and Glow Discharges (GD), are recognized as valuable tools for inorganic analyses. ${ }^{1,2}$ Although significant differences are apparent between ICPs, GDs, and other plasma sources, all are prone to spectral interferences. Mass spectral interferences are caused by inter-element isotope overlaps, residual and plasma gases, and combinations of these with analyte species $\left(\mathrm{eg} \mathrm{Ar}^{+}, \mathrm{MAr}^{+}, \mathrm{M}_{2}^{+}, \mathrm{Ar}_{2}\right)^{3,}{ }^{4}$ Interferences are even more problematic when complex matrices are analyzed, eg. biological samples and soils. ${ }^{5-7}$ Because quantitative analyses can be severely hampered by such interferences, methods are sought to reduce the spectral impact of these compounds.

The ideal approach to removing isobaric interferences arising from polyatomic species would be non-selective with respect to interelement treatment, yet highly selective in the attenuation of polyatomic species. At present there is much research into ion-molecule reactions to eliminate the adverse effects of polyatomic interferences. These include 1) selective adduct formation of the atomic ions of interest (such as the conversion of $\mathrm{Ce}^{+}$to $\mathrm{CeO}^{+}$using molecular oxygen for the determination of $\mathrm{Ce}^{8}$ ); 2) charge neutralization of polyatomic interferences; ${ }^{8-13}$ 3) adduct formation of interferences; ${ }^{14,15}$ and 4) CID of molecular interferences. ${ }^{13,16,17}$ 
Collision/reaction cells are receiving much attention due to their ability to achieve chemical resolution prior to mass analysis. Typically, a small mass bandpass is used so that undesirable reaction products are continuously ejected from the reaction cell. ${ }^{18,} 19$ Ultimately, such reactions can be highly specific and this means that an independent 'recipe' exists for the determination of a certain element in the presence of a certain interference. Also, cluster formation is often observed for some elements ${ }^{8}$ (such as $\mathrm{Fe}^{+}$ with ammonia reagent gas) and this can further complicate the spectra. Ideally, a lessselective attenuation scheme, such as CID is more desirable.

The use of CID for the dissociation of diatomic and polyatomic ions has been pursued for atomic ion sources including ICPs and GDs. ${ }^{14,16,17,20}$ Application of quadrupole collision cells has been reasonably successful in this regard. At best, atomic ion losses are always noted to beat a minimum of $50 \%$ loss in atomic ion signal. This level of analyte loss is not acceptable for trace determinations. ${ }^{8}$ In order to achieve greater dissociation rates for polyatomics in linear quadrupoles, one must either increase the number of collisions with the target gas atoms, or increase the (center of mass) energy of the collisions. The latter experiment is achieved by increasing the acceleration voltage leading to the collision cell, but the increased energies often result in increased scattering. To increase the number of collisions, one must either increase the residence time of the ion in the collisional environment, or increase the pressure. The residence time is not freely adjustable in linear quadrupoles, because the time spent in each quadrupole is a function of the kinetic energy of the ion and the cell length. Increasing the pressure deteriorates the transmission and mass resolution due to increased scattering collisions. In quadrupole ion traps, however, increasing the trapping time of the ion easily increases the 
number of collisions between the ion and the target gas. Therefore, although the QIT utilizes center-of-mass collision energies lower than those in linear quadrupoles, the increased residence time makes CID ultimately a much more complete process. $^{21,22}$ Another advantage of QITs is that the acceleration of a particular trapped ion is dependent upon the amplitude and frequency of the excitation voltage. This excitation frequency can selectively accelerate ions of a particular $\mathrm{m} / \mathrm{z}$ while trapping the remaining ions at near-thermal conditions, whereas in linear quadrupoles, all the ions are accelerated by the bias-voltage of the collision cell. The QIT is therefore a more selective method of promoting CID of molecules.

Evidence has been presented that CID in QITs might be a possible approach to removing molecular interferences while minimizing analyte ion losses at the same nominal mass. ${ }^{23}$ When measured independently and under identical excitation conditions, the dissociation rate of ${ }^{181} \mathrm{Ta}^{16} \mathrm{O}^{+}$was found to be considerably greater than the scattering rate of ${ }^{197} \mathrm{Au}^{+}{ }^{23}$ This difference arises because the potential well depth and collision time of QITs is much larger than the well depth and residence time in linear quadrupoles. This particular case serves as an interesting model because the tantalum oxide ion has a very strong bond dissociation energy $\left(\sim 750 \mathrm{~kJ} \mathrm{~mol}^{1}\right) .^{24}$

As well as presenting evidence that when ${ }^{181} \mathrm{Ta}^{16} \mathrm{O}^{+}$and ${ }^{197} \mathrm{Au}^{+}$are simultaneously subjected to resonance excitation the dissociation rate of ${ }^{181} \mathrm{Ta}^{16} \mathrm{O}^{+}$exceeds the scattering rate of ${ }^{197} \mathrm{Au}^{+}$, this paper also considers two more examples of isobaric interference. In these cases isotope ratios are used to verify the low scattering rates of the atomic ion species. Evidence is also presented to show that the diatomic ion interferences can actually be attenuated during the scan function of the acquisition table, viz. the isobars 
can be removed as the mass spectrum is collected. This implies that no operator intervention is required to enable the removal of isobaric interferences, and that this approach should also be applicable to the removal of interferences across the entire mass spectral range.

\subsection{Experimental}

A pulsed dc glow discharge, similar in design to that described previously, ${ }^{25,} 26$ was used as the ion source. To generate tantalum oxide ions in the presence of gold ions, tantalum metal powder was first pressed into a gold pin that served as the cathode. Neon was used as the discharge gas at 1 Torr and as the QIT bath gas at 0.5 mTorr (corrected). Ion manipulation using filtered-noise-fields (FNF frequencies $)^{27}$ was used to obtain either ${ }^{181} \mathrm{Ta}^{16} \mathrm{O}^{+}$or a mixture of ${ }^{181} \mathrm{Ta}^{16} \mathrm{O}^{+}$and ${ }^{197} \mathrm{Au}^{+}$at mass 197 , as follows. To obtain ${ }^{181} \mathrm{Ta}^{16} \mathrm{O}^{+}$at mass 197 in the absence of ${ }^{197} \mathrm{Au}$, all the ions of mass $181\left(\mathrm{Ta}^{+}\right)$and 197 $\left({ }^{181} \mathrm{Ta}^{16} \mathrm{O}^{+}\right.$and $\left.{ }^{197} \mathrm{Au}^{+}\right)$were collected during the ionization table. The ions at $\mathrm{m} / \mathrm{z} 197$ were then resonantly ejected at a $\mathrm{q}_{\mathrm{z}}=0.67$ with an excitation voltage $\sim 500 \mathrm{mV}_{\mathrm{pp}}$ at 226 $\mathrm{kHz}$. The tantalum ions were then allowed to form ${ }^{181} \mathrm{Ta}^{16} \mathrm{O}^{+}$by reacting them with molecular oxygen admitted to the trapping region at a pressure of $\sim 1 \times 10^{-6}$ Torr. The unreacted tantalum ions remaining after $80 \mathrm{~ms}$ were resonantly ejected leaving ${ }^{181} \mathrm{Ta}^{16} \mathrm{O}^{+}$ ions as the only surviving species at $\mathrm{m} / \mathrm{z}$ 197. If the exact procedure is followed, with the exception of the first isolation step, one obtains the same number of ${ }^{181} \mathrm{Ta}^{16} \mathrm{O}^{+}$ions at mass 197 with the addition of a) the ${ }^{181} \mathrm{Ta}^{16} \mathrm{O}^{+}$ions formed in the glow discharge and trapped during the ionization table and b) the ${ }^{197} \mathrm{Au}^{+}$ions. 
To measure the CID rate, as described previously, ${ }^{28,29}$ resonance excitation at 226 $\mathrm{kHz}$ frequency was applied at a $\mathrm{q}_{\mathrm{z}}=0.67$. A second frequency was simultaneously applied for ejection of the $\mathrm{Ta}^{+}$product ions to prevent the forward reaction. Mass spectra obtained at various excitation times allowed the determination of the dissociation rate for ${ }^{181} \mathrm{Ta}^{16} \mathrm{O}^{+}$. The same approach was applied in the presence of ${ }^{197} \mathrm{Au}^{+}$to determine the effect of the excitation voltage on the scattering rate of $\mathrm{Au}^{+}$.

To dissociate the isotopologues of $\mathrm{GdO}^{+}$in the presence of ytterbium ions, a small amount of isotopically enriched ( 50:50 $\left.{ }^{154} \mathrm{Gd}:{ }^{155} \mathrm{GD}\right) \mathrm{Gd}_{2} \mathrm{O}_{3}$ was pressed on to a $\mathrm{Yb}$ metal pin. Dual GD pulses were used in each scan in order to obtain the desired ratios of $\mathrm{GdO}^{+}$to $\mathrm{Yb}^{+}$. In the first pulse, FNF waveforms were used to selectively trap the bare $\mathrm{Gd}^{+}$ions. The second pulse was used to add the desired amount of $\mathrm{Yb}^{+}$and subsequent reactions of $\mathrm{Gd}^{+}$with added oxygen $\left(\sim 1.6 \times 10^{-6}\right.$ Torr $)$ formed the required interferences. The $\mathrm{Yb}^{+}$ion abundance remains virtually unchanged during this time because its reaction with $\mathrm{O}_{2}$ is endothermic. ${ }^{30}$ Ions between $\mathrm{m} / \mathrm{z}$ 165-180 were then isolated and cooled at a $\mathrm{q}_{\mathrm{z}} \approx 0.3$ for $20 \mathrm{~ms}$. The method of dissociation in this case involved a fixed frequency excitation voltage of $370 \mathrm{mV}_{\mathrm{pp}}$ at $226 \mathrm{kHz}$ with different drive amplitudes. In this way, ions of different $\mathrm{m} / \mathrm{z}$ are sequentially brought in to resonance with the excitation voltage and the degree of dissociation/scattering can be evaluated for each $\mathrm{m} / \mathrm{z}$.

To dissociate copper dimers in the presence of tellurium a small quantity of tellurium oxide was pressed onto the surface of a copper pin. At high glow discharge voltages $(\sim 1500 \mathrm{~V}$ at $\sim 1$ Torr $)$ quantities of $\mathrm{Cu}_{2}{ }^{+}$and $\mathrm{Te}$ could be generated in appropriate ratios for this study. After isolation and cooling, a broad range of $60 \mathrm{mV}_{\mathrm{pp}}$ amplitude voltages were applied over the m/z range $120-140(96-112 \mathrm{KHz}$ at $1 \mathrm{KHz}$ 
increments), so as to cause all the ions to undergo excitation simultaneously. Because the copper ions are so much lighter than the dimer ions, the low-mass cut-off value of 44.7 amu was used in order to ensure that the bare copper ions were recaptured after dissociation. If the dimer ions were trapped at a low mass cut-off greater than $63 \mathrm{amu}$ during excitation, the bare copper products would not be recaptured from the dissociation event.

\subsection{Results and discussion}

\subsection{1. ${ }^{181} \mathrm{Ta}^{16} \mathrm{O}^{+}$in the presence of ${ }^{197} \mathrm{Au}{ }^{+}$}

Tantalum monoxide interference in the measurement of gold atomic ions was chosen as the initial case study for this investigation. Tantalum oxide is readily formed in the trap and has a particularly strong bond, $\sim 750 \mathrm{~kJ} \mathrm{~mol}^{-1} \cdot{ }^{24}$ It is therefore difficult to quantitatively dissociate using linear quadrupole systems. ${ }^{17}$ Values of the integrated peak area at different times were used to study the effects of resonance excitation on the dissociation and scattering rates of ${ }^{181} \mathrm{Ta}^{16} \mathrm{O}^{+}$and ${ }^{197} \mathrm{Au}^{+}$respectively. Figure 7.1.a shows the ion signals obtained when ${ }^{181} \mathrm{Ta}^{16} \mathrm{O}^{+}$is dissociated at $320 \mathrm{mV}_{\mathrm{pp}}$ in the absence of ${ }^{197} \mathrm{Au}^{+}$. In this example the ${ }^{181} \mathrm{Ta}^{+}$product ion is not ejected during the dissociation event resulting in the reformation of ${ }^{181} \mathrm{Ta}^{16} \mathrm{O}^{+}$from the ${ }^{181} \mathrm{Ta}^{+} \mathrm{CID}$ product. 

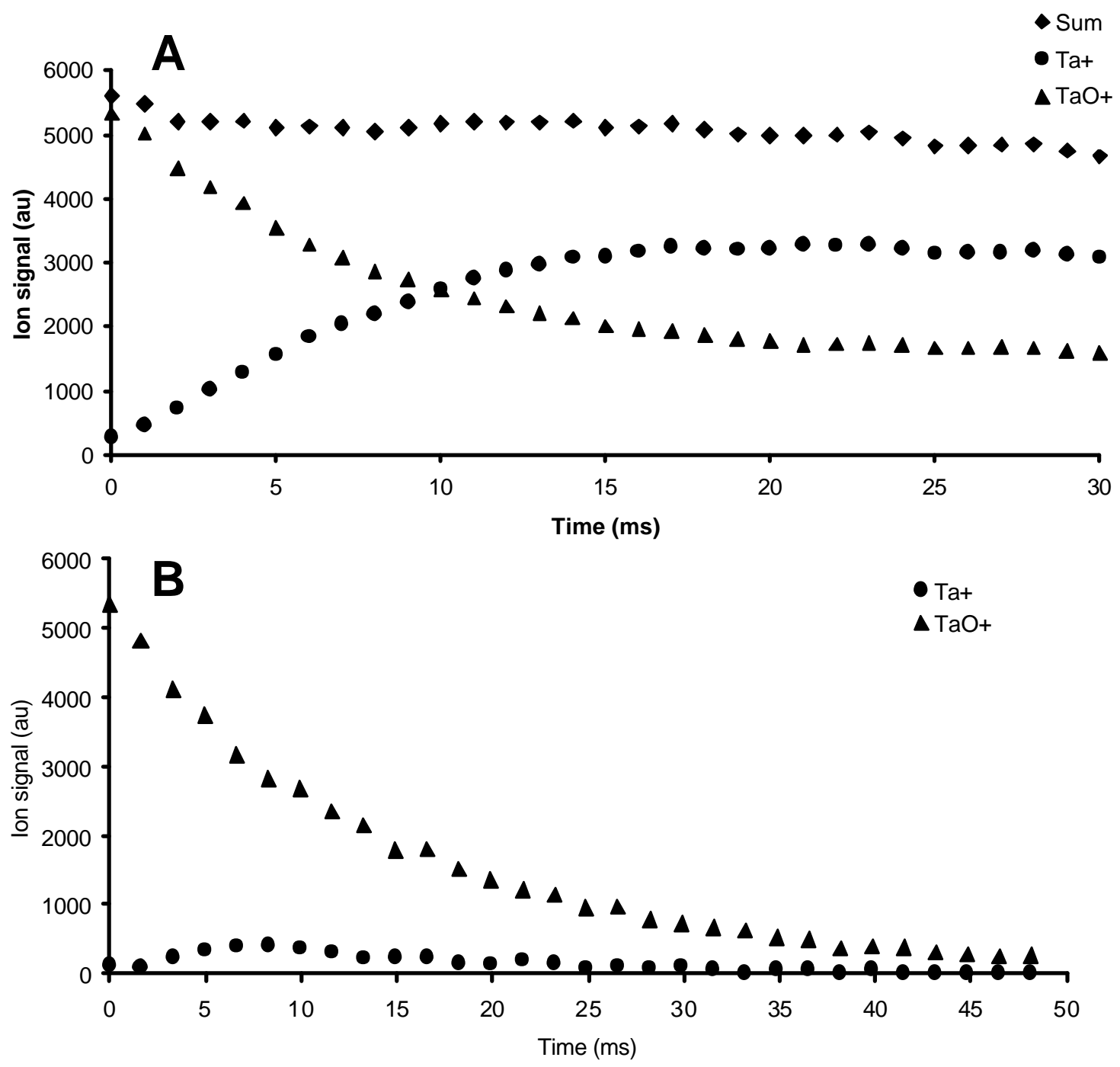

Figure 7.1. a) Ion signals obtained at $320 \mathrm{mV}_{\mathrm{pp}}$ excitation amplitude, $\mathrm{Ta}^{+}$not ejected and b) Ion signals obtained at $250 \mathrm{mV}_{\mathrm{pp}}$ with resonance ejection on $\mathrm{Ta}^{+}$. 
The total ion current shows that $<100 \%$ recovery is achieved at this voltage when ${ }^{181} \mathrm{Ta}^{+}$ is not resonantly ejected during the dissociation table. Better trapping efficiencies are possible at lower excitation amplitudes, with a concomitant decrease in dissociation rate, but even with no excitation voltage applied, the trapping efficiencies were slightly less than $100 \%$ at $30 \mathrm{~ms}$. The small losses in total ion signal after $30 \mathrm{~ms}$ indicates the occurrence of scattering, or of ion molecule reactions resulting in ions outside the massrange of the trapping conditions.

After $20 \mathrm{~ms}$, steady state signals are obtained for the dissociation rate and the forward oxidation rate. The relative position of the 'equilibrium' signals can be shifted by altering the excitation frequency: higher excitation voltages increase the dissociation rate and promote the abundance of ${ }^{181} \mathrm{Ta}^{+}$over ${ }^{181} \mathrm{Ta}^{16} \mathrm{O}^{+}$(this will be discussed in Figure 7.2). Figure 7.1.b shows that when a simultaneous resonant ejection voltage is applied to the ${ }^{181} \mathrm{Ta}^{+}$product ion, it can be efficiently ejected from the trap. A plot of $-\ln \left(\left[\mathrm{Ta}^{+}\right]_{\mathrm{t}} /\left[\mathrm{Ta}^{+}\right]_{\mathrm{t} 0}\right)$, where $\left[\mathrm{Ta}^{+}\right]_{\mathrm{t}}$ is the ion signal at time $\mathrm{t}$ and $\left[\mathrm{Ta}^{+}\right]_{\mathrm{t} 0}$ is the ion signal at time zero, versus time gives a linear plot $^{28}$ with a phenomenological dissociation rate of $68 \mathrm{~s}^{-1}$ at $250 \mathrm{mV}_{\mathrm{pp}}$.

Figure 7.2 shows various ion signals as a function of time obtained when ${ }^{181} \mathrm{Ta}^{16} \mathrm{O}^{+}$is dissociated in the presence of ${ }^{197} \mathrm{Au}^{+}$. The ${ }^{181} \mathrm{Ta}^{+}$is not coincidentally ejected during the dissociation event in these cases. The ${ }^{197} \mathrm{Au}^{+}$signal makes up approximately $60 \%$ of the signal at $\mathrm{m} / \mathrm{z} 197$. When the higher excitation voltage of $530 \mathrm{mV}_{\mathrm{pp}}$ is applied the equilibrium concentration of ${ }^{181} \mathrm{Ta}^{+}$increases compared to the value at $320 \mathrm{mV}_{\mathrm{pp}}$. This is due to the increased dissociation rate at higher excitation amplitudes in balance with the constant forward oxidation rate. At both voltages, the total ion current decreases by $\sim 5 \%$ over $30 \mathrm{~ms}$, again, indicating a small degree of scattering or ion-molecule 
reaction losses. Close inspection of the total ion signals in Figure 7.2 reveals that the ion losses are the same, $\sim 5 \mathrm{~s}^{-1}$, regardless of the excitation amplitude. This indicates that the losses are independent of the excitation voltage, and no additional scattering is caused by the applied excitation frequencies. Thus, appreciable dissociation of tantalum oxide is possible $\left(\sim 70 \mathrm{~s}^{-1}\right)$ with no detectable scattering of the gold ions caused by the excitation voltage.

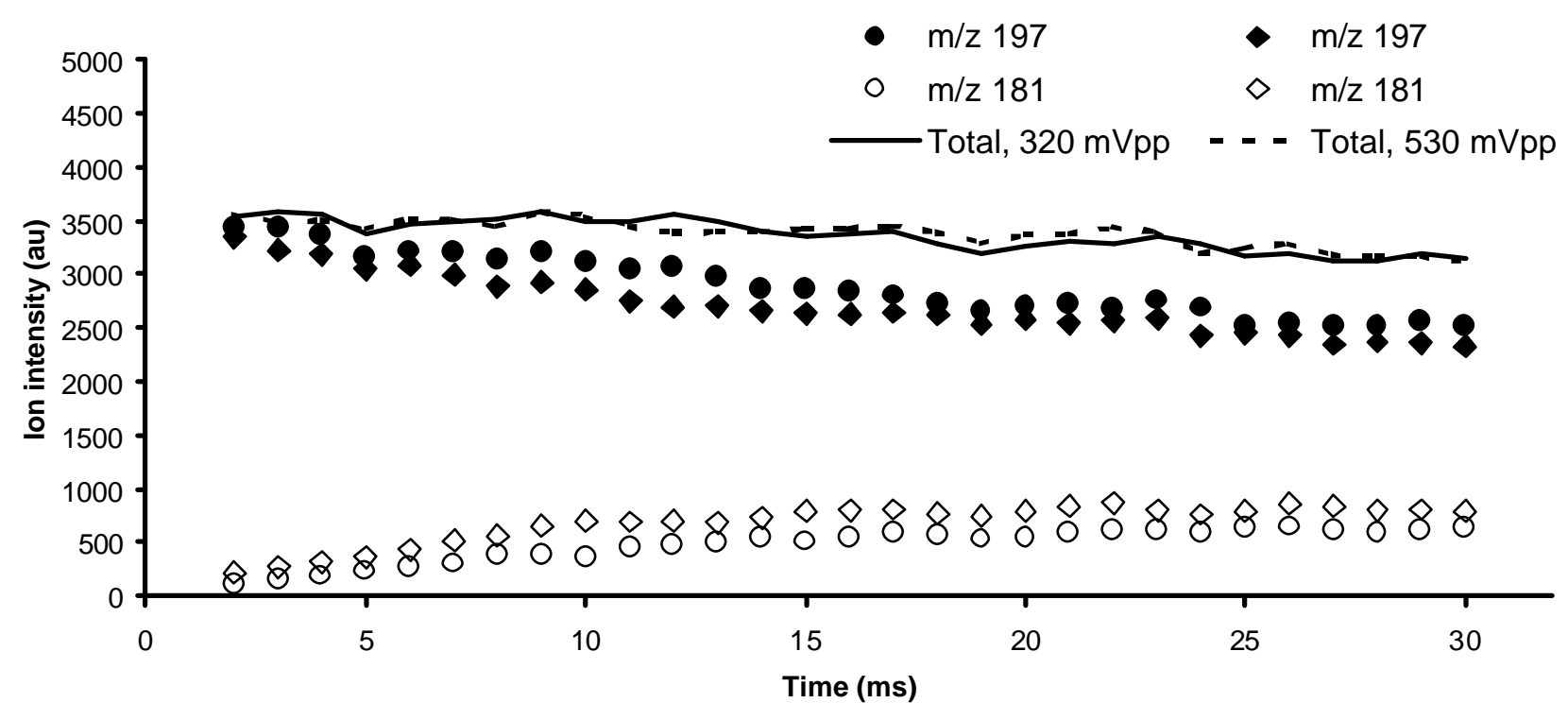

Figure 7.2. Plot of ion signal versus excitation time to show the effect of excitation amplitude on the steady state signals of ${ }^{181} \mathrm{Ta}^{+}$at $\mathrm{m} / \mathrm{z} 181$ and ${ }^{181} \mathrm{Ta}^{16} \mathrm{O}^{+} /{ }^{197} \mathrm{Au}^{+}$at $\mathrm{m} / \mathrm{z} 197$. 
In order to prevent the ${ }^{181} \mathrm{Ta}^{16} \mathrm{O}^{+}$continually forming from the ${ }^{181} \mathrm{Ta}^{+}$, the experiment was repeated with a resonance ejection voltage on $\mathrm{m} / \mathrm{z} 181$ during the dissociation step. The experiment was performed first with only ${ }^{181} \mathrm{Ta}^{16} \mathrm{O}^{+}$ions present and then with ${ }^{181} \mathrm{Ta}^{16} \mathrm{O}^{+}$and ${ }^{197} \mathrm{Au}^{+}$present. The number density of ${ }^{181} \mathrm{Ta}^{16} \mathrm{O}^{+}$ions in each experiment remained virtually constant because the amount of ${ }^{181} \mathrm{Ta}^{16} \mathrm{O}^{+}$formed during the ionization table is small. The ion signal at $\mathrm{m} / \mathrm{z} 197$ is plotted as a function of time in Figure 7.3 with and without the presence of ${ }^{197} \mathrm{Au}^{+}$. It is clear that the rate of ion loss at $\mathrm{m} / \mathrm{z} 197$ is very similar in each case. If one plots the difference between the two ion signals a steady state value is obtained. With the evidence provided in Figure 2 - that the ${ }^{181} \mathrm{Ta}^{16} \mathrm{O}^{+}$readily dissociates and that the scattering loss of ${ }^{197} \mathrm{Au}^{+}$is $<5 \mathrm{~s}^{-1}$ - the difference between the two slopes in Figure 7.3 can be attributed to the signal of ${ }^{197} \mathrm{Au}^{+}$. The 'steady state' signal also decreases at a rate of approximately $5 \mathrm{~s}^{-1}$ and is similar to the ion loss rate obtained in the absence of an excitation voltage or ${ }^{197} \mathrm{Au}^{+}$. The observation that the ion losses are no greater in this experiment than in the absence of ${ }^{197} \mathrm{Au}^{+}$indicates that almost no ${ }^{197} \mathrm{Au}^{+}$are scattered during this time. The attenuation rate for ${ }^{197} \mathrm{Au}^{+}$ions is therefore at least an order of magnitude smaller than the attenuation rate of the isobaric ${ }^{181} \mathrm{Ta}^{16} \mathrm{O}^{+}$ions $\left(68 \mathrm{~s}^{-1}\right)$, and small relative to the omnipresent ion loss rate of $5 \mathrm{~s}^{-1}$. In both cases, signals due to ${ }^{181} \mathrm{Ta}^{16} \mathrm{O}^{+}$are reduced to nearly zero after $20-30 \mathrm{~ms}$, with no detectable loss of ${ }^{197} \mathrm{Au}^{+}$, when present. 


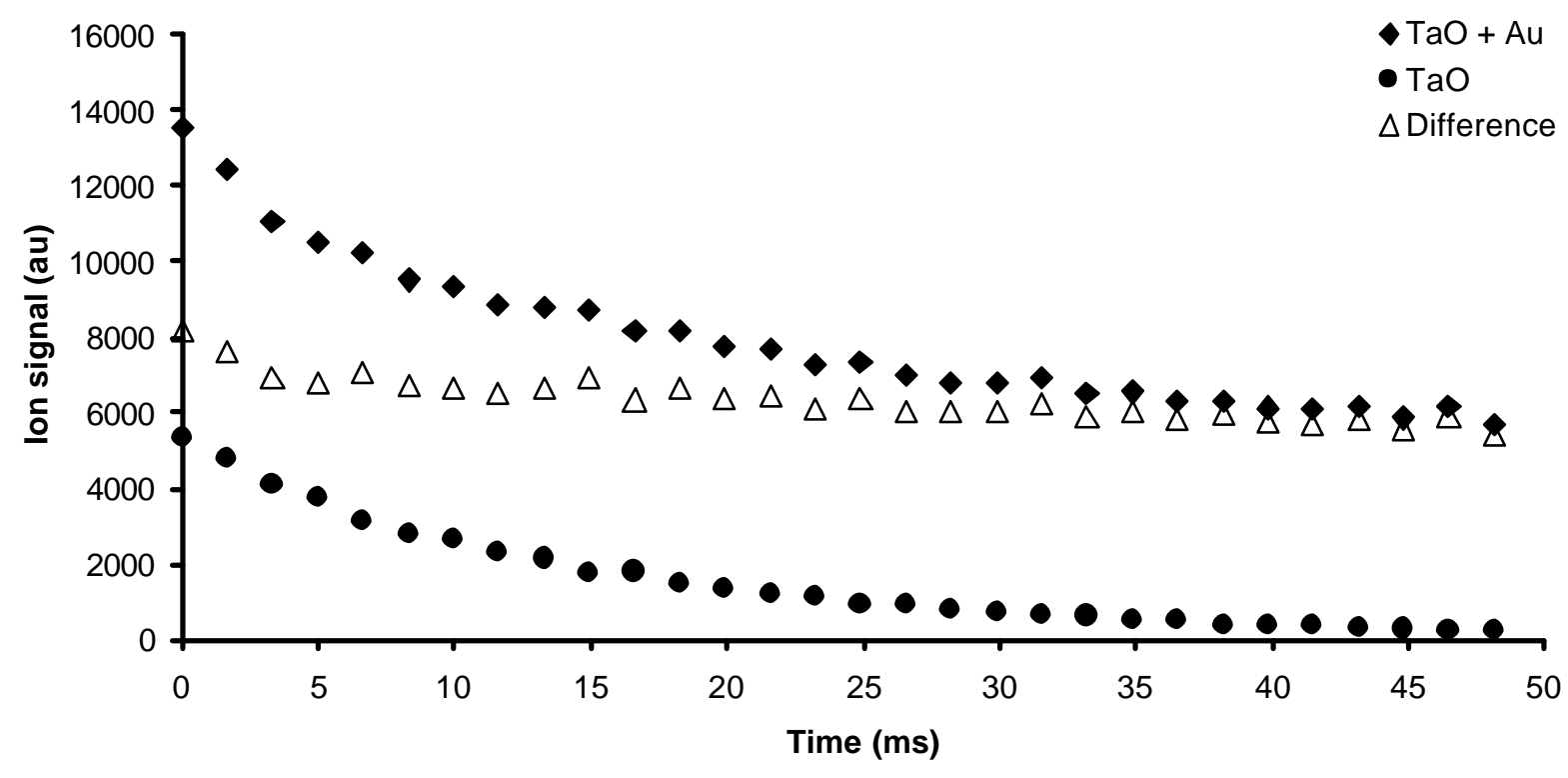

Figure 7.3. Plot of the ion signal versus time to show the attenuation rate at $\mathrm{m} / \mathrm{z} 197$ with and without the presence of $\mathrm{Au}^{+}$. 
7.3.2. ${ }^{154} \mathrm{Gd}^{16} \mathrm{O}^{+}$and ${ }^{155} \mathrm{Gd}^{16} \mathrm{O}^{+}$in the presence of ${ }^{170} \mathrm{Yb}^{+}$and ${ }^{171} \mathrm{Yb}^{+}$

Further evidence for the preferential and efficient trapping of an atomic ion at conditions necessary for dissociating a diatomic ion at the same mass can be gained by using elements and interferences with multiple isotopes. Figure 7.4.a shows a mass spectrum of the isotopes of $\mathrm{Yb}^{+}$with interfering isotopologues of $\mathrm{GdO}^{+}$present. $\mathrm{GdO}^{+}$ ions have a high dissociation energy of approximately $735 \mathrm{~kJ} \mathrm{~mol}^{1},{ }^{31}$ and again represent a particularly stubborn molecular interference. Figures 7.4.b and 7.4.c are examples of two mass spectra obtained using a supplementary excitation amplitude of $400 \mathrm{mV}_{\mathrm{pp}}$ at $226 \mathrm{kHz}$. In this case, the low mass cut-off value (drive amplitude) is steadily increased in the experimental editor in order to sweep the different masses into resonance with the applied frequency. 

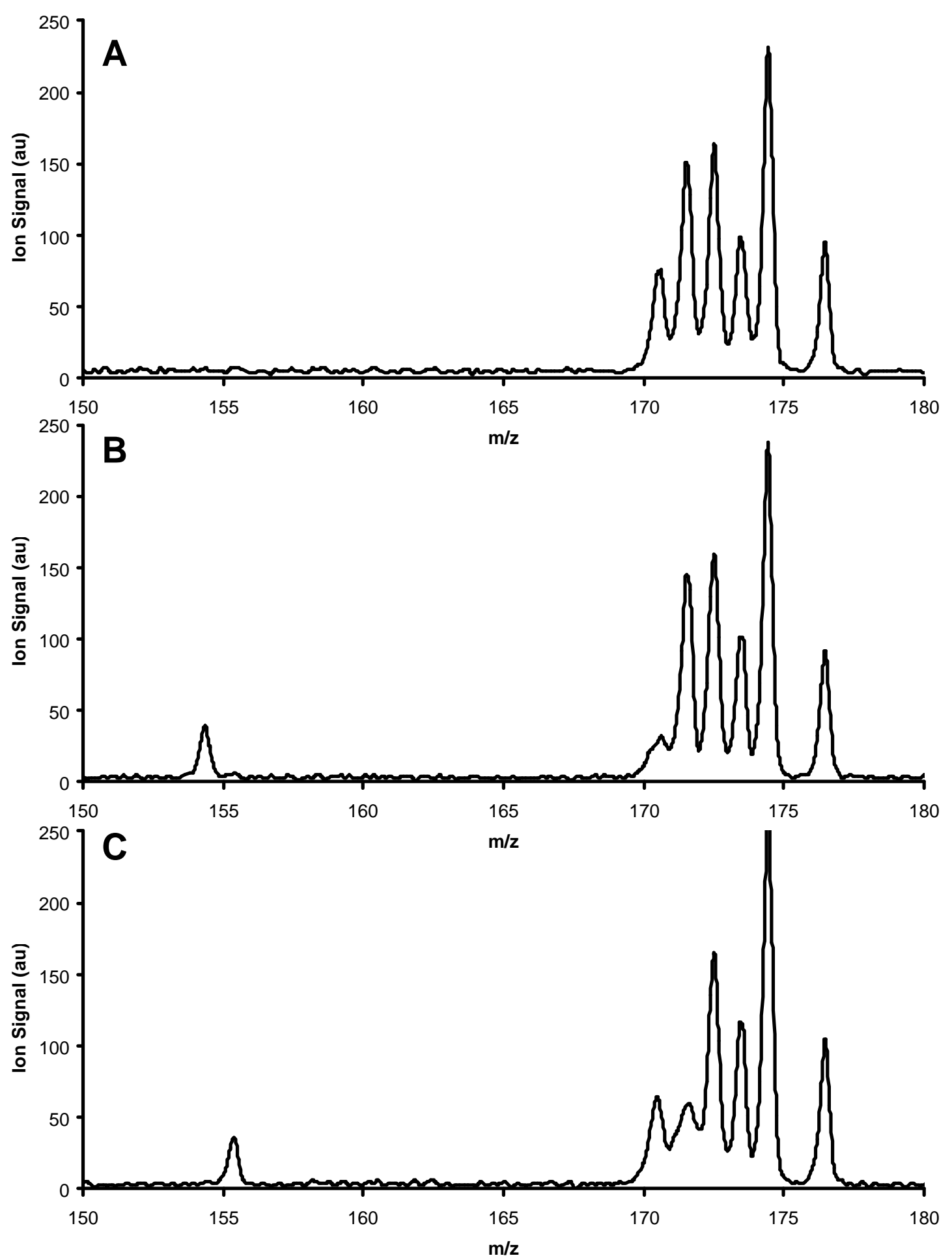

Figure 7.4. a) Isotopes of $\mathrm{Yb}^{+}$with $\mathrm{GdO}^{+}$interfering at $\mathrm{m} / \mathrm{z} 170$ and $171, \mathrm{~b}$ ) single frequency excitation on m/z 170 and c) single frequency excitation on m/z 171 . Excitation amplitude of $370 \mathrm{mV}_{\mathrm{pp}}$ at $226 \mathrm{kHz}$ for $20 \mathrm{~ms}$ in $0.5 \mathrm{mTorr} \mathrm{Ne}$. 
In Figure 7.4.b, the excitation frequency is on-resonance with $\mathrm{m} / \mathrm{z} 170$. The ${ }^{154} \mathrm{Gd}^{16} \mathrm{O}^{+}$ions are quantitatively dissociated, while the bare ${ }^{170} \mathrm{Yb}^{+}$ions are retained. The same effect is seen in Figure 7.4.c when the excitation voltage comes into resonance with $\mathrm{m} / \mathrm{z} 171$.

This experiment provides evidence that it is possible to sweep the dissociating voltage over a specified mass range in order to provide attenuation of multiple isobaric interferences. This method has the benefit that the dissociation of interferences at each $\mathrm{m} / \mathrm{z}$ can be swept through the excitation frequency so that excitation occurs when the excited ions are held at the largest potential well depth. This allows stronger excitation amplitudes to be applied, ${ }^{32}$ allowing the dissociation of stronger bonds, while ensuring that the bare metal ions are retained in the trap.

7.3.3. ${ }^{63,63} \mathrm{Cu}_{2}{ }^{+}{ }^{63,65} \mathrm{Cu}_{2}{ }^{+}$, and ${ }^{65,65} \mathrm{Cu}_{2}{ }^{+}$in the presence of ${ }^{126} \mathrm{Te}^{+},{ }^{128} \mathrm{Te}^{+}$, and ${ }^{130} \mathrm{Te}^{+}$

Instead of sweeping all the masses through a single frequency excitation signal, one might also envisage using a broadband excitation to dissociate interferences over a wide mass range. This could conceivably be carried out using random noise ${ }^{33}$ supplementary frequencies, but this approach is not investigated here. A broadband approach using a fixed trapping potential would restrict the size of the potential well depth of each mass being excited. Based on the Mathieu stability diagram, lighter ions will generally be held in a larger potentiat-well-depth than heavier ions. The lighter ions are therefore capable of obtaining larger kinetic energies before they overcome the well depth and are scattered. ${ }^{32}$ Over small mass ranges $(\sim 20 \mathrm{~m} / \mathrm{z})$, the well-depth differences are small, and presumably would not adversely affect these experiments. 
An example of the broadband approach is given in Figure 7.5, for the dissociation of copper dimers in the presence of tellurium atoms. ${ }^{4}$ Figure $7.5 . a$ shows the mass spectrum of Te ions being interfered by copper dimers. Isotopic analysis of the peaks was made and compared with the natural isotopic abundance of tellurium in Table 7.1. Before the spectrum is freed from molecular interferences, the apparent isotope ratios are particularly poor, especially for the major isotopes at m/z 126, 128 and 130.

Figure 7.5.b shows a mass spectrum acquired after a $20 \mathrm{~ms}$ multiple-frequency excitation at $60 \mathrm{mV}_{\mathrm{pp}}$ at $\mathrm{q}_{\mathrm{z}} \approx 0.3$. The dissociation of $\mathrm{Cu}_{2}{ }^{+}$at this low trapping potential is made possible by the ease at which the dimer dissociates: its dissociation energy has been estimated at $\sim 170 \mathrm{~kJ} \mathrm{~mol}^{-1} .{ }^{34}$ The two isotopes of copper at $\mathrm{m} / \mathrm{z} 63$ and 65 are retained in the trap and display a reasonably accurate isotopic ratio (see Table 7.1). Note that the uninterfered Te ions are not scattered, even though they are exposed to the same excitation amplitude as the interfered isotopes. This is clearly evident in Table 7.1 where the isotopic distribution for the tellurium ions is now much closer to the expected distribution. The isotopic ratios for tellurium in the complete absence of $\mathrm{Cu}_{2}{ }^{+}$has not been measured using this ion trap, so mass biases have not been accounted for. This experiment shows that not only can the atomic tellurium spectra be resolved from the interfering dimers of copper using CID, but the fragmentation products of the CID process - the bare copper ions_ — can also be retained for isotopic analysis. 

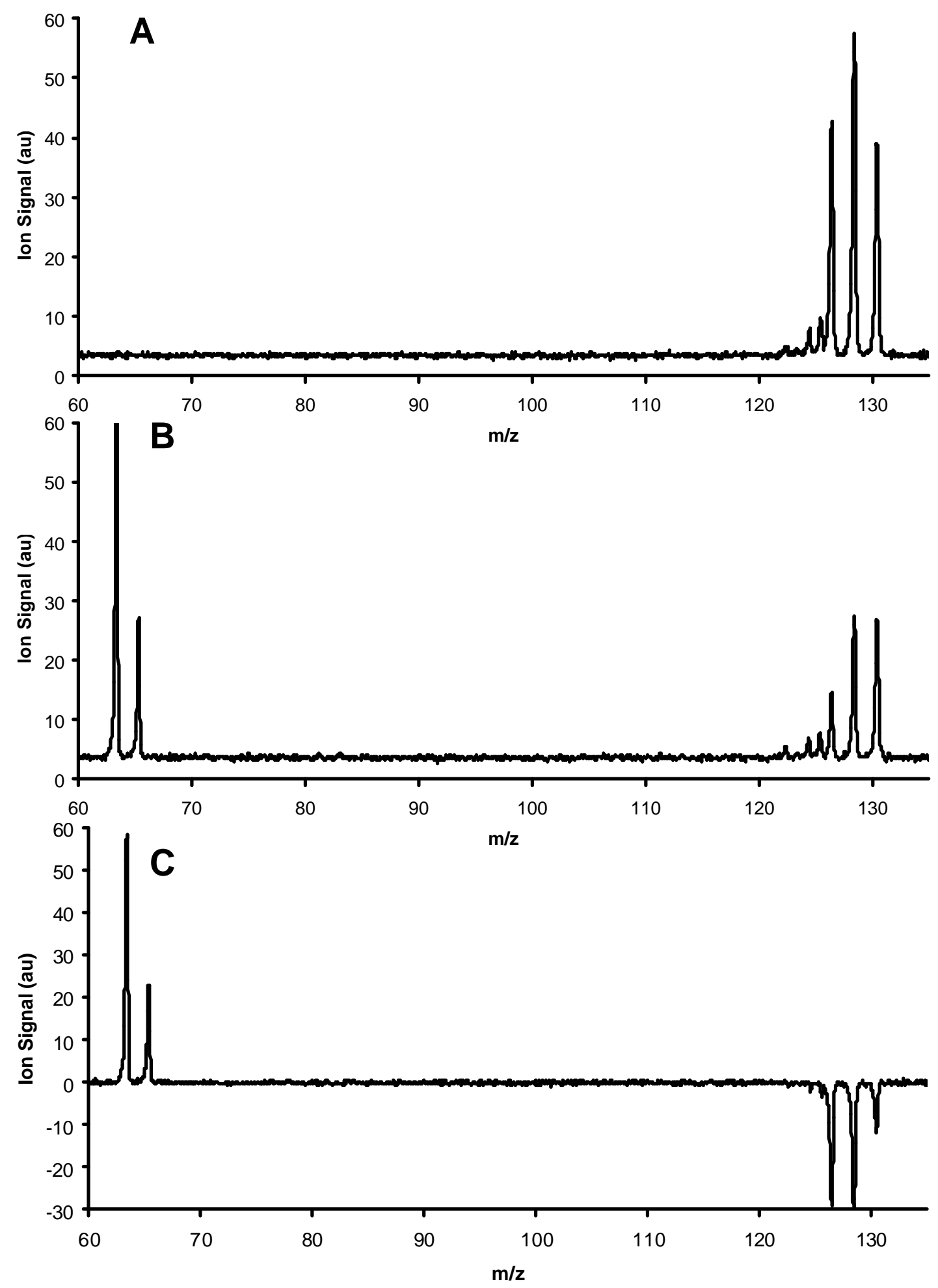

Figure 7.5. Broadband dissociation of $\mathrm{Cu}_{2}{ }^{+}$isotopologues in the presence of $\mathrm{Te}^{+}$isotopes:

A) $20 \mathrm{~ms}, 0 \mathrm{~V}_{\mathrm{pp}}$ applied, B) $20 \mathrm{~ms}, 60 \mathrm{mV}_{\mathrm{pp}}$ on each frequency over the range $\mathrm{m} . \mathrm{z}$ 120-140; and C) difference spectrum between Figures A and B to show the quantitative recapture of dissociated $\mathrm{Cu}$ ions. 
Table 7.1. Measured isotopic abundances for some Te ions before and after the application of broadband excitation to remove interfering $\mathrm{Cu}_{2}{ }^{+}$ions.

\begin{tabular}{|c|c|c|c|c|}
\hline \multirow[b]{2}{*}{$\mathrm{m} / \mathrm{z}$} & \multirow{2}{*}{$\begin{array}{l}\text { Interference } \\
\text { before CID }\end{array}$} & \multicolumn{2}{|c|}{ Relative abundances* $(\%)$} & \multirow{2}{*}{$\begin{array}{l}\text { Natural isotopic } \\
\text { abundance }^{35}(\%)\end{array}$} \\
\hline & & Before CID & After CID & \\
\hline 63 & & I & 72.7 & 69.2 \\
\hline 65 & & l & 27.3 & 30.8 \\
\hline 122 & & 1.9 & 2.8 & 2.6 \\
\hline 124 & & 4.2 & 6.2 & 4.9 \\
\hline 125 & & 6.0 & 7.1 & 7.1 \\
\hline 126 & ${ }^{63} \mathrm{Cu}_{2}{ }^{+}$ & 26.6 & 16.6 & 19.0 \\
\hline 128 & ${ }^{63} \mathrm{Cu}^{65} \mathrm{Cu}^{+}$ & 36.1 & 33.0 & 31.7 \\
\hline 130 & ${ }^{65} \mathrm{Cu}_{2}{ }^{+}$ & 25.1 & 34.4 & 33.8 \\
\hline
\end{tabular}

*Calculated from peak areas.

Figure 7.5.c is the difference between Figures 7.5.a and 7.5.b and demonstrates that if this procedure was carried out in a multielement standard over a wide mass range it would be possible to determine the precursors to certain fragment ions. In this example it is clear that the isotopic distribution of the copper dimer ions between $\mathrm{m} / \mathrm{z} 126-130$ produces the isotopic distribution observed at $\mathrm{m} / \mathrm{z} 63$ and 65 (Table 7.1). Although the sum of the peak heights at m/z 63 and 65 in Figure 7.5.c appear to be larger than the sum of the dimer ion peaks, integration of the peak areas reveals that charge is indeed conserved. The lower mass peaks are simply narrower.

Finally, we were interested to see if the copper dimers could be dissociated during the acquisition period of the scan cycle. This was achieved by applying a fixed frequency excitation at a lower frequency than the acquisition frequency at $451 \mathrm{kHz}$. This method is 
similar to axial modulation that can be used to achieve mass-range extension. ${ }^{36-38}$ In these experiments, however, the additional supplementary frequency in the scan table is not sufficient to eject the ions, but is large enough to kinetically excite them. This technique is shown schematically in Figure 7.6 for a diatomic ion in which the constituent atoms have different masses.

In part 7.6.A, the diatomic is stable in the trap under normal trapping conditions. As the drive amplitude is increased to eject sequentially the lowest mass ions for detection, ions successively come into resonance with the supplementary frequency. This is shown in part B of the sequence in Figure 7.6. During on-resonance excitation, the diatomic is kinetically excited by the supplementary frequency and undergoes collisions with the bath gas. If enough energy is transferred from these collisions to internal energy in the ion, the ion can dissociate. The charge will be carried away by one of the atoms; the other will be neutral and therefore not retained. The charged ion will have a lower $\mathrm{m} / \mathrm{z}$ than the original diatomic, and will be trapped closer to the edge of the stability diagram, as shown in part $\mathrm{C}$. 


\section{Supplementary}

excitation

A

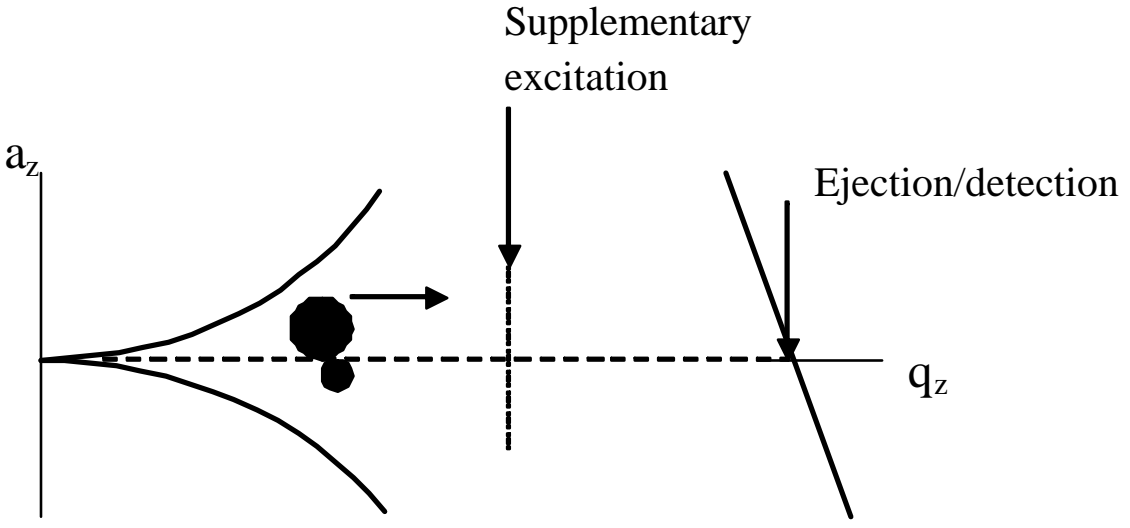

B

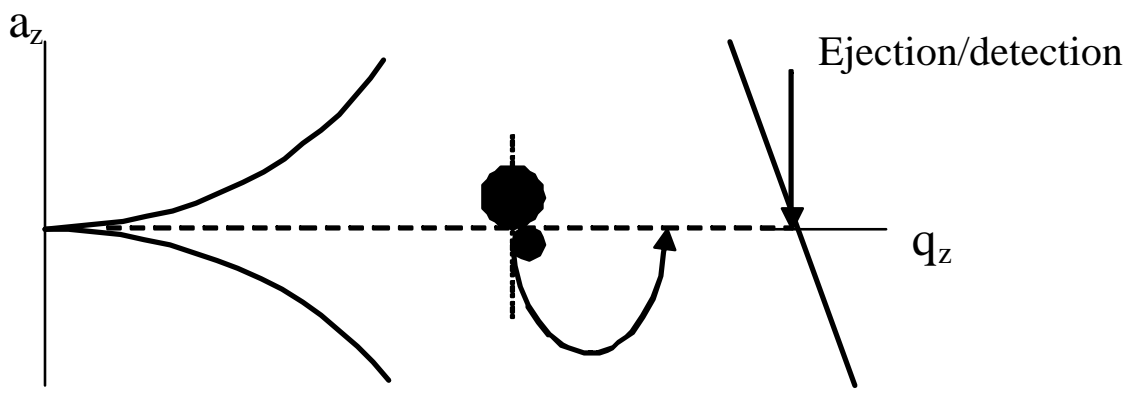

$\mathbf{C}$

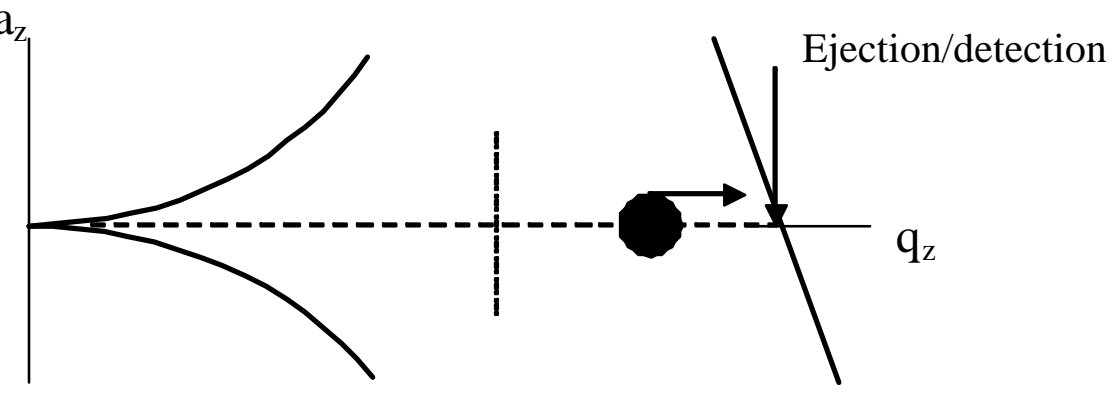

Fig. 7.6. Schematic to represent the CID of a diatomic ion using a supplementary excitation frequency with axial modulation. A) diatomic is stable in trap and not in resonance with the supplementary excitation frequency. The increasing driveamplitude sweeps the ion towards the edge of the instability diagram. B) Diatomic comes into resonance with the supplementary frequency. CID is achieved. C) Only the charged dissociation products are trapped after CID, and are closer to the stability diagram boundary than the original diatomic; they are ejected at the edge of the stability boundary and detected. 
Atomic ions at the same nominal mass as the diatomic will be kinetically excited by the supplementary voltage, but because they cannot undergo dissociation, they will kinetically cool when the increasing drive amplitude sweeps them out of resonance again. They will remain trapped at a lower $\mathrm{q}_{\mathrm{z}}$ value than the lighter ions and will be ejected later in the axial modulation table. Therefore, as long as the product ions do not fall at a $\mathrm{q}_{\mathrm{z}}$ greater than that at the edge of the stability boundary (0.91), the masses of the dissociation products (carrying the charge) can be determined. For copper dimer ions, each isotope has an equal probability of taking away the charge in the dissociation reaction, which gives rise to the correct observed isotope ratios at $\mathrm{m} / \mathrm{z} 63$ and 65 . For metal oxides, the bare metal products will be the final charge carriers and vacuum system will pump away the oxygen.

Figure 7.7 shows the mass spectrum obtained when a $400 \mathrm{mV}_{\mathrm{pp}}$ voltage at 110 $\mathrm{kHz}$ is applied while the acquisition frequency at $451 \mathrm{kHz}$ is ramped in amplitude. The very small hump at $\mathrm{m} / \mathrm{z} 47$ reveals the time at which the dimer at $\mathrm{m} / \mathrm{z} 126$ first comes in to resonance with the dissociation voltage. The peak shows that the dimer ions are slightly scattered during the time they are being heated. The ramp rate used in this experiment was $3000 \mathrm{amu} \mathrm{s}^{-1}$ (normal ramp rates are $\sim 12000 \mathrm{amu} \mathrm{s}^{-1}$ ) and this was close to the slowest possible ramp rate allowed by the software. This gives each copper dimer isotope approximately $0.3 \mathrm{~ms}$ of on-resonance excitation. Clearly, to obtain a greater degree of dissociation and less scattering, a slower acquisition speed and smaller voltage is required so that each isotope remains on-resonance for a longer time. However, because our current software restricts the scan speed, we are currently adapting another instrument to be able to perform these experiments and increase CID efficiencies. 
By using a slower drive-amplitude ramp rate it should be possible to dissociate isobaric interferences during the acquisition ramp of the ion trap. As higher masses come into resonance with the excitation frequency it might also be necessary to simultaneously ramp the amplitude of the excitation frequency. This is because in ion trap CID experiments heavier ions require larger excitation voltages than lighter ions, even when the dissociation energies are the same (see Chapter 6). ${ }^{39}$

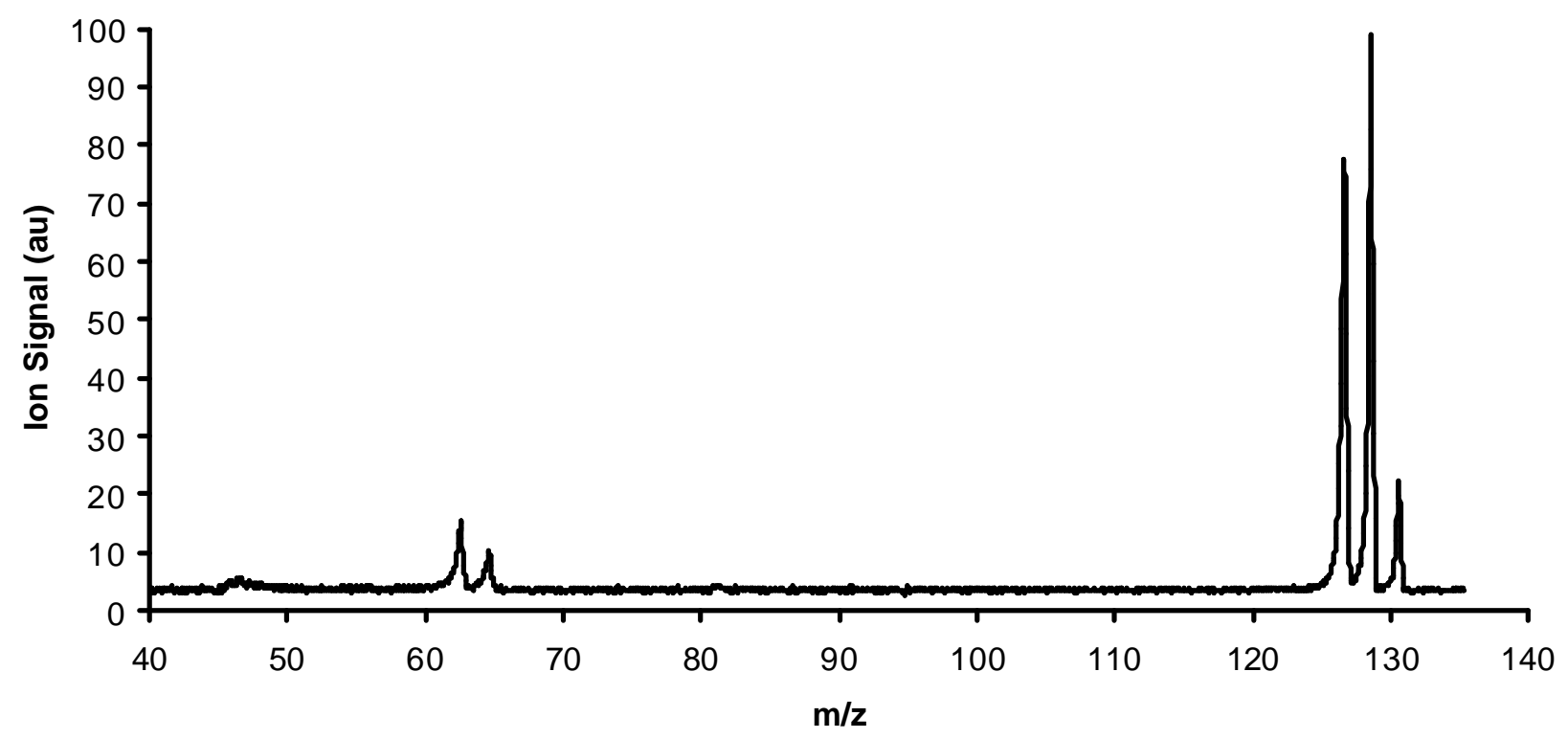

Figure 7.7. Mass spectrum obtained when $\mathrm{Cu}_{2}{ }^{+}$is subjected to resonance excitation at $110 \mathrm{kHz}$ during axial modulation. Each isotope of the dimer ion is on-resonance for approximately $0.3 \mathrm{~ms}$. 


\subsection{Conclusions}

Evidence is provided here that CID in QITs provides a universal (non-element specific) means of reducing polyatomic isobaric interferences for the determination of atomic mass spectra. If desired, fragments of the CID process can be trapped for detection, or ejected, depending on whether those fragments are of analytical importance. Several different methods for applying dipolar excitation have been accomplished including single-frequency-excitation fixed-trapping-potential, multiple-frequency excitation fixed-trapping-potential, and single-frequency-excitation variable-trappingpotential waveforms. The latter provides a method for achieving CID during the acquisition ramp that generates the mass spectrum.

Further work is planned to incorporate a system in which certified standards are available to validate the claims of the usefulness of this technique for elemental analysis, and to determine isotopic abundances with greater accuracy. An example would be certified zinc standards, with isotopes of vanadium oxide interfering at m/z 66 and 67. 


\subsection{References}

(1) Barshick, C. M. In Inorganic Mass Spectrometry; Barshick, C. M., Duckworth, D. C., Smith, D. H., Eds.; Marcel Dekker, Inc.: New York, 2000; Vol. 23.

(2) Olesik, J. W. In Inorganic Mass Spectrometry; Barshick, C. M., Duckworth, D. C., Smith, D. H., Eds.; Mercel Dekker, Inc: New York, 2000.

(3) Barshick, C. M.; Smith, D. H.; Johnson, E.; King, F. L.; Bastug, T.; Fricke, B. Appl. Spec. 1995, 49, 885-889.

(4) Shao, Y.; Horlick, G. Spectrochim. Acta, Part B 1991, 46, 165-174.

(5) Vanhoe, H.; Goossens, J.; Moens, L.; Dams, R. J. Anal. At. Spectrom. 1994, 9 , $177-185$.

(6) Baude, S.; Broekaert, J. A. C.; Delfosse, D.; Jakubowski, N.; Fuechtjohann, L.; Orellana-Velado, N. G.; Pereiro, R.; Sanz-Medel, A. J. Anal. At. Spectrom. 2000, $15,1516-1525$.

(7) Duckworth, D. C.; Barshick, C. M.; Bostick, D.; Smith, D. H. Appl. Spec. 1992, $47,243-245$.

(8) Douglas, D. J. Can. J. Spectrosc. 1989, 34, 38-49.

(9) Rowan, J. T.; Houk, R. S. Appl. Spectrosc. 1989, 43, 976-980.

(10) Koppenaal, D. W.; Barinaga, C. J.; Smith, M. R. J. Anal. At. Spectrom. 1994, 9, 1053-1058.

(11) Eiden, G. C.; Barinaga, C. J.; Koppenaal, D. W. J. Anal. At. Spectrom. 1996, 11, $317-322$. 
(12) Eiden, G. C.; Barinaga, C. J.; Koppenaal, D. W. J. Am. Soc. Mass Spectrom. 1996, 7, 1161-1171.

(13) Duckworth, D. C.; Eyler, J. R.; Watson, C. H. In Inorganic Mass Spectrometry.; Barshick, C. M., Duckworth, D. C., Smith, D. H., Eds.; Marcel Dekker, Inc.: New York, 2000, pp 329.

(14) Duckworth, D. C.; Barshick, C. M. Anal. Chem. 1998, 70, 709A-717A.

(15) Irikura, K. K.; Fowles, E. H.; Beauchamp, J. L. Anal. Chem. 1994, 66, 3447.

(16) Duckworth, D. C.; Marcus, R. K. Appl. Spectrosc. 1990, 44, 649-655.

(17) King, F. L.; Harrison, W. W. Int. J. Mass Spectrom. Ion. Processes 1989, 89, 171185.

(18) Eiden, G. C.; Barinaga, C. J.; Koppenaal, D. W. Rapid Commun. Mass Spectrom. 1997, 11, 37-42.

(19) Eiden, G. C.; Barinaga, C. J.; Koppenaal, D. W. J. Anal. At. Spectrom. 1999, 14, $1129-1132$.

(20) Mei, Y.; Duckworth, D. C.; Cable, P. R.; Marcus, R. K. J. Am. Soc. Mass Spectrom. 1994, 5, 845-851.

(21) McLuckey, S. A. J. Am. Soc. Mass Spectrom. 1991, 3, 599-614.

(22) McLuckey, S. A.; Goeringer, D. E. J. Mass Spectrom. 1997, 32, 461-474.

(23) Duckworth, D. C.; McLuckey, S. A., 26th Federation of Analytical Chemistry and Spectroscopy Societies Conference, Vancouver, Canada, October 1999.

(24) Calculated using the equation $B D E\left(T a O^{+}\right)=B D E(T a O)+I E(T a)-I E(T a O)$. Value for $B D E(T a O)=799 \mathrm{~kJ} \mathrm{~mol}^{-1}$ is taken from CRC Handbook of Chemistry and Physics.; 81 ed.; Lide, D. R., Ed.; CRC Press: New York, 2000, and values 
for IE $(\mathrm{Ta})=714 \mathrm{~kJ} \mathrm{~mol}^{-1}$ and $\mathrm{IE}(\mathrm{TaO})=764 \mathrm{~kJ} \mathrm{~mol}^{-1}$ are taken from S. G. Lias, J. E. Bartmess, J. F. Liebman, J. L. Holmes, P. D. Levin, W. G. Millard, J. Phys. Chem. Ref. Data, 17 (1988), Sup. 1.

(25) Duckworth, D. C.; Smith, D. H.; McLuckey, S. A. J. Anal. At. Spectrom. 1997, $12,43-48$.

(26) McLuckey, S. A.; Glish, G. L.; Duckworth, D. C.; Marcus, R. K. Anal. Chem. 1992, 64, 1606-1609.

(27) Goeringer, D. E.; Asano, K. G.; McLuckey, S. A.; Hoekman, D.; Stiller, S. W. Anal. Chem. 1994, 66, 313-318.

(28) Duckworth, D. C.; Goeringer, D. E.; McLuckey, S. A. J. Am. Soc. Mass Spectrom. 2000, 11, 1072-1078.

(29) Jackson, G. P.; King, F. L.; Goeringer, D. E.; Duckworth, D. C. Int. J. Mass Spectrom. 2002, 216, 85-93.

(30) Koyanagi, G. K.; Bohme, D. K. J. Phys. Chem. A 2001, 105, 8964-8968.

(31) Chandrasekharaiah, M. S.; Gingerich, K. A. In Handbook on the Physics and Chemistry of the Rare Earths; K. A. Gschneidner, J., Eyring, L., Eds.; Elsevier: New York, 1989; Vol. 12, pp 409-430.

(32) Charles, M. J.; McLuckey, S. A.; Glish, G. L. J. Am. Soc. Mass Spectrom. 1994, $5,1031-1041$.

(33) McLuckey, S. A.; Goeringer, D. E.; Glish, G. L. Anal. Chem. 1992, 64, 14551460.

(34) Radzig, A. A.; Smirnov, B. M. Reference data on atoms, molecules and ions; Springer: Berlin, 1985. 
(35) Lide, D. R., Ed. CRC Handbook of Chemistry and Physics., 81 ed.; CRC Press: New York, 2000.

(36) Stafford, G. C.; Kelly, P. E.; Syka, J. E. P.; Reynolds, W. E.; Todd, J. F. J. Int. J. Mass Spectrom. Ion Processes 1984, 60, 85-98.

(37) Todd, J. F. J.; Penman, A. D. Int. J. Mass Spectrom. Ion Processes 1991, 106, 120.

(38) Kaiser, R. E.; Louris, J. N.; Amy, J. W.; Cooks, R. G. Rapid Commun. Mass Spectrom. 1989, 3, 225-230.

(39) Jackson, G. P.; King, F. L.; Goeringer, D. E.; Duckworth, D. C. Int. J. Mass Spectrom. 2001, In press. 


\section{G L E N P . JACKSO N}

8/ 1998 - present West Virginia University

Morgantown, WV, USA

Ph.D. Analytical Chemistry (expected 8/ 2002)

- Developed and characterized methods for reducing molecular interferences in optical and mass spectrometric analyses in glow discharge spectroscopies.

8/ 1996 - 7/ 1997 Ohio University

Athens, OH, USA

M.S. Analytical Chemistry (received 6/ 1998)

- Studies completed as part of a "year abroad" exchange program with B.S. degree at University of Wales Swansea.

- Developed a fast screening technique for onganochlorine pesticides in water samples using various extraction methods and fast GC.

9/ 1994 - 6/ 1998 University of Wales Swansea Swansea, Wales, UK B.S. Chemical and Analytical Science (received 7/ 1998)

Final project: Characterization of a gas-monitoring $\mathrm{CO}_{2}$ optical sensor.

- ASMS Travel Award, East Tennessee Mass Spectrometry Discussion Group, 5/ 2001.

- Forrest Ferrel Award, WVU Chemistry Department, 5/ 2000.

- HERF Fellowship Award, West Vinginia University, 8/ 1999-2000.

- Outstanding TA of the Year award, Chemistry Department, West Vinginia University, 5/ 1999.

- HERF Fellowship Award, West Virginia University, 8/ 1998-1999. 
5/ 2000 - present Oak Ridge N ational Laboratory Oak Ridge, TN

Ph.D. Research Internship

- Ion-molecule reactions in quadrupole ion traps.

8/ 1998 - 5/ 2000 West Virginia University

Morgantown, WV

Ph.D. Research and Teaching Assistant

- Optical spectroscopic and time-of-flight mass spectrometric studies of pulsed glow discharges.

8/ 1996 - 7/ 1997 Ohio University

Athens, $\mathbf{0 H}$

M.S. Research and Teaching Assistant

- Fast extraction methods coupled with fast gas chromatography for screening of organochlonine pesticides in water.

PROFESSIONAL MEMBERSHIPS AND POSITIONS OF RESPON SIBILITY

- American Chemical Society

- Royal Society of Chemistry (MRSC designation)

- American Society for Mass Spectrometry

- Secretary elect for 2003, East Tennessee Mass Spectrometry Discussion Group

- Phi Lambda Upsilon (Honorary Chemistry Society)

- Board of Govemors, West Virginia Wine and Jazz Festival, 11/ 1999$10 / 2000$

- Executive Committee Press and Publicity Officer, University of Wales Swansea Athletic Union, 9/ 1997-6/ 1998

- Kayak Club President, University of Wales Swansea, 9/ 1997-6/ 1998 
G. P. Jackson, F. L. King, "E ffect of Nitrogen on a Millisecond Pulsed Glow Discharge in Argon", Submitted to Spectrochim. Acta, Part B.

G. P. Jackson, J. K. Gibson, D. C. Duckworth, "Gas-Phase Reactions of Bare and Oxo-Ligated Actinide and Lanthanide Cations with Pentamethylcyclopentadiene in a Q uadrupole Ion Trap Mass Spectrometer", Int. J. Mass Spectrom. In press

G. P. Jackson, F. L. King, D. E. Goeringer, D. C. Duckworth, “Gas-Phase Reactions of $\mathrm{U}^{+}$and $\mathrm{U}^{2+}$ with $\mathrm{O}_{2}$ and $\mathrm{H}_{2} \mathrm{O}$ in a Quadrupole Ion Trap", J. Phys. Chem. A. In Press

G. P. Jackson, F. L. King, D. E. Goeninger, D. C. Duckworth, "Collisioninduced dissociation of lanthanide oxide ions in quadrupole ion traps: effects of bond strength and mass", Int. J. Mass Spectrom. 216(1) (2002) 85-93.

G. P. Jackson, C. Lewis, S. K. Doom, V. Majidi, F. L. King, "Spectral, Spatial and Temporal Diagnostics of a Millisecond Pulsed Glow D ischarge: Argon Atom Metastables", Spectrochim. Acta, Part B 56(12) (2001) 2449-2464.

C. Lewis, G. P. Jackson, S. K. Doom, D. Wayne, V. Majidi, F. L. King, "Spectral, Spatial and Temporal Diagnostics of a Millisecond Pulsed Glow Discharge: Copper Atom and Ion Signals", Spectrochim. Acta, Part B 56(5) (2001) 487-501.

G. P. Jackson, A. R. J. Andrews, "N ew Fast Screening Method for Organochlonine Pesticides in Water by Using Solid-Phase Microextraction with Fast Gas Chromatography and a Pulsed-Discharge Electron Capture Detector", Analyst 123(5) (1998) 1085-1090.

PRESE NTAT IONS (Presenter listed first)

G. P. Jackson, F. L. King, "Post-Pulse Ion Generation in a Millisecond Pulsed Glow Discharge,", 2002 Winter Conference on Plasma Spectrochemistry, Scottsdale, AZ, 1/ 2002.

D. C. Duckworth, D. E. Goeninger, G. P. Jackson, F. L. King, "Breaking Up and Making Up: Gas-Phase Heavy Metal Ion Chemistry In Q uadrupole Ion Traps", $28^{\text {th }}$ Federation of Analytical Chemistry and Spectroscopy Societies Conference, Detroit, MI, 10/ 2001 .

F. L. King, G. P. Jackson, L. Lei, C. L.ewis, T. Millay, M. A. Moser, V. Majidi, "Excitation And Ionization In a MS Pulsed Glow D ischarge Plasma: Implications For Atomic And Molecular 
Determinations" 28 $^{\text {th }}$ Federation of Analytical Chemistry and Spectroscopy Societies Conference, Detroit, MI, 10/ 2001.

L. L. Gdovka, G. P. Jackson, "Beckett's Fin de Partie and E instein's Theory of Relativity", $26^{\text {th }}$ Colloquium on Literature and Film, West Virginia University, Morgantown, WV, 9/ 2001.

D. C. D uckworth, G. P. Jackson, F. L. King, "Glow Discharge Quadrupole Ion Trap Mass Spectrometry: Analytical Tool, Chemical Probe, Or Mere Curiosity?", 53rd Annual Meeting of the Southeastem Region of the American Chemical Society, Savannah, GA, 9/ 2001 .

G. P. Jackson, F. L. King, D. E. Goeringer, D. C. D uckworth, "Determination of Mass Effects on the Dissociation of Diatomic Ions in a Quadrupole Ion Trap", Poster presented at $49^{\text {th }}$ ASMS Conference on Mass Spectrometry and Allied Topics, Chicago, IL, 5/ 2001.

D. C. D uckworth, G. P. Jackson, F. L. King, "Mechanistic and Kinetic Investigation of the Oxidation of Uranium Ions in a Q uadrupole Ion Trap" 49 $^{\text {th }}$ ASMS Conference on Mass Spectrometry and Allied Topics, Chicago, IL, 5/ 2001.

F. L. King, C. Lewis, G. P. Jackson, "2-D Spatial and Temporal Mapping of MS-Pulsed DC and RF Glow Discharges" $2^{27^{\text {th }}}$ Federation of Analytical Chemistry and Spectroscopy Societies Conference, Nashville, T , 9/ 2000.

G. P. Jackson, F. L. King, D. C. Duckworth, "Relative Dissociation Rate Measurements of Rare Earth Element Oxides in a Q uadrupole Ion Trap Mass Spectrometer", $27^{\text {th }}$ Federation of Analytical Chemistry and Spectroscopy Societies Conference, $N$ ashville, TN, 9/ 2000.

G. P. Jackson, C. Lewis, F. L. King, "Two-Dimensional Optical Investigation of a Millisecond Pulsed Glow Discharge Source", $27^{\text {th }}$ Federation of Analytical Chemistry and Spectroscopy Societies Conference, Nashville, T N, 9/ 2000.

D. C. Duckworth, J. K. Gibson, G. P. Jackson, F. L. King, "Reactions of Gas-Phase Uranium with Pentamethylcyclopentadiene in a Q uadrupole Ion Trap", $48^{\text {th }}$ ASMS Conference on Mass Spectrometry and Allied Topics, Long Beach, CA, 6/ 2000.

G. P Jackson, C.Lewis, S. K. Doom, V. Majidi, F. L. King, "Temporally and Spatially Resolved Diagnostics of a Pulsed Glow Discharge Source", 2000 Winter Conference on Plasma Spectrochemistry, Fort Lauderdale, FL, 1/ 2000. 
G. P Jackson, C.Lewis, S. Doom, V. Majidi, F. L. King, " Temporally and Spatially Resolved Diagnostics of Pulsed Glow Discharge Sources" , 52nd Southeast Regional American Chemical Society Meeting, Knoxville, TN, 10/ 1999.

S. K. Doorn, G. P. Jackson, C. Lewis, D. Wayne, V. Majidi, F. L. King, "Diagnostics of a Pulsed Glow Discharge Source", Poster presented at $26^{\text {th }}$ Federation of Analytical Chemistry and Spectroscopy Societies Conference, Vancouver, Canada, 10/ 1999.

A. R. J. Andrews, L. de Jager, H. Zhang, G. P. Jackson, "Fast Extraction of Chloninated Pesticides from Water Samples", $25^{\text {th }}$ Federation of Analytical Chemistry and Spectroscopy Societies Conference, Austin, TX, 10/ 1998.

A. R. J. Andrews, G. P. Jackson, "Fast Screening of Water Samples for Chlorinated Pesticides," 24 ${ }^{\text {th }}$ Federation of Analytical Chemistry and Spectroscopy Societies Conference, Providence, RI, 10/ 1997.

G. P. Jackson, A. R. J. Andrews, "Chlorinated Pesticide Determination by Solid-Phase Microextraction and Fast Gas Chromatography", Pittsburgh Conference, Atlanta, GA, 3/ 1997. 